Comparações de populações discretas

Alexandre Hiroshi Watanabe 



\title{
Comparações de populações discretas
}

\author{
Alexandre Hiroshi Watanabe
}

\section{Orientador:Prof. Dr. Dorival Leão Pinto Júnior}

Dissertação apresentada ao Instituto de Ciências Matemáticas e de Computação - ICMC - USP, como parte dos requisitos para obtenção do título de Mestre em Ciências - Ciências de Computação e Matemática Computacional. VERSÃO REVISADA

USP - São Carlos

Maio de 2013 
Ficha catalográfica elaborada pela Biblioteca Prof. Achille Bassi e Seção Técnica de Informática, ICMC/USP, com os dados fornecidos pelo(a) autor(a)

W324c

Watanabe, Alexandre Hiroshi

Comparações de Populações Discretas / Alexandre Hiroshi Watanabe; orientador Dorival Leão Pinto Júnior. -- São Carlos, 2013.

$$
109 \mathrm{p} \text {. }
$$

Dissertação (Mestrado - Programa de Pós-Graduação em Ciências de Computação e Matemática

Computacional) -- Instituto de Ciências Matemáticas e de Computação, Universidade de São Paulo, 2013.

1. $\mathrm{CE} 632.7 .23 .2 . \mathrm{CE} 632.7 .9 .3 . \mathrm{CE} 632.7 .39 .4$. CE632.7.13. 5. CE631.7.36. I. Pinto Júnior, Dorival Leão, orient. II. Título. 
À minha família

Fernanda Shigeeda, Letícia Rika Shigeeda Watanabe e Lucas Seiji Shigeeda Watanabe. 



\section{Agradecimentos}

Agradeço à minha esposa Fernanda Shigeeda e meus filhos Letícia e Lucas pela companhia e ter me alegrado nos momentos mais difíceis da minha vida.

Aos meus pais, Shuji Watanabe e Sonoe Masago Watanabe por todo apoio e carinho por todos esses anos. Aos meus irmãos Miyuki e Yukio também vai um agradecimento especial.

Ao meu orientador Dorival Leão pela dedicação e paciência na condução deste árduo trabalho, pela amizade, conselhos e ensinamentos que tomarei como lição por toda a vida.

Aos meus amigos Vinícius Melo, Vinícius "Cabeça”, Daniel "Bona", Rafael Geraldo, os alunos da Mat 05 em especial os "Kamissamas" Willian, Vânia, Aline e Márcia pelo companheirismo, ajuda nesses anos acadêmicos.

Aos professores do ICMC - USP, em especial, Ires, Janete, Valdemir pelo apoio e conhecimento nos anos de graduação.

Ao CNPq pelo apoio financeiro e finalmente à Universidade de São Paulo que me proporcionou uma excelente formação. 



\section{Resumo}

Um dos principais problemas em testes de hipóteses para a homogeneidade de curvas de sobrevivência ocorre quando as taxas de falha (ou funções de intensidade) não são proporcionais. Apesar do teste de Log-rank ser o teste não paramétrico mais utilizado para se comparar duas ou mais populações sujeitas a dados censurados, este teste apresentada duas restrições. Primeiro, toda a teoria assintótica envolvida com o teste de Log-rank, tem como hipótese o fato das populações envolvidas terem distribuições contínuas ou no máximo mistas. Segundo, o teste de Log-rank não apresenta bom comportamento quando as funções intensidade cruzam. O ponto inicial para análise consiste em assumir que os dados são contínuos e neste caso processos Gaussianos apropriados podem ser utilizados para testar a hipótese de homogeneidade. Aqui, citamos o teste de Renyi e Cramér-von Mises para dados contínuos (CCVM), ver Klein e Moeschberger (1997) [15]. Apesar destes testes não paramétricos apresentar bons resultados para dados contínuos, esses podem ter problemas para dados discretos ou arredondados.

Neste trabalho, fazemos um estudo simulação da estatística de Cramér von-Mises (CVM) proposto por Leão e Ohashi [16], que nos permite detectar taxas de falha não proporcionais (cruzamento das taxas de falha) sujeitas a censuras arbitrárias para dados discretos ou arredondados. Propomos também, uma modificação no teste de Log-rank clássico para dados dispostos em uma tabela de contingência. Ao aplicarmos as estatísticas propostas neste trabalho para dados discretos ou arredondados, o teste desenvolvido apresenta uma função poder melhor do que os testes usuais.

Palavras-chave: Log-rank Ponderado, Log-rank Modificado, Cramér-von Mises, Dados Discretos, Simulação Monte Carlo, Cruzamento de taxas de falha, Censura. 



\section{Abstract}

One of the main problems in hypothesis testing for homogeneity of survival curves occurs when the failure rate (or intensity functions) are nonproportional. Although the Log-rank test is a nonparametric test most commonly used to compare two or more populations subject to censored data, this test presented two constraints. First, all the asymptotic theory involved with the Log-rank test, is the hypothesis that individuals and populations involved have continuous distributions or at best mixed. Second, the log-rank test does not show well when the intensity functions intersect.

The starting point for the analysis is to assume that the data is continuous and in this case suitable Gaussian processes may be used to test the assumption of homogeneity. Here, we cite the Renyi test and Cramér-von Mises for continuous data (CCVM), and Moeschberger see Klein (1997) [15]. Despite these non-parametric tests show good results for continuous data, these may have trouble discrete data or rounded.

In this work, we perform a simulation study of statistic Cramér-von Mises (CVM) proposed by Leão and Ohashi [16], which allows us to detect failure rates are nonproportional (crossing of failure rates) subject to censure for arbitrary data discrete or rounded. We also propose a modification of the test log-rank classic data arranged in a contingency table. By applying the statistics proposed in this paper for discrete or rounded data, developed the test shows a power function better than the usual testing.

Keywords: Weighted Log-rank, Modified Log-rank, Cramér-von Mises, Discrete Data, Monte Carlo simulation, Crossing hazard, Censorship. 



\section{Lista de Figuras}

1.1 Produto limite estimado da distribuição da sobrevivência. . . . . . . . . . . . . . . 3

1.2 Gráfico dos alunos em cada uma das classes desde a última ocorrência. . . . . . . . . . . . 4

1.3 Função de Intensidade para o tempo até a evasão dos alunos ingressantes por vestibular. . . 5

4.1 Fluxograma da Simulação. . . . . . . . . . . . . . . . . . . . . . 31

4.2 Gráfico da simulação sob a hipótese nula $H_{0}$ (sem censura) e espaço finito. . . . . . . . . 33

4.3 Gráfico da Função Poder do teste para 2 populações, tamanho de amostra 500 e sem censura. 35

4.4 Gráfico da Função Poder do teste para 3 populações, tamanho de amostra 500 e sem censura. 36

4.5 Gráfico da Função Poder do teste para 5 populações, tamanho de amostra 500 e sem censura. 37

4.6 Gráfico da Função Poder do teste para 2 populações, $\delta$ de 0,05 e sem censura. . . . . . . . 39

4.7 Gráfico da Função Poder do teste para 3 populações, $\delta$ de 0,05 e sem censura. . . . . . . . 40

4.8 Gráfico da Função Poder do teste para 5 populações, $\delta$ de 0,05 e sem censura. . . . . . . . 41

4.9 Gráfico da Função Poder do teste para 2 populações e sem censura. . . . . . . . . . . . . . 43

4.10 Gráfico da Função Poder do teste para 3 populações e sem censura. . . . . . . . . . . . . . 44

4.11 Gráfico da Função Poder do teste para 5 populações e sem censura. . . . . . . . . . . . . . 45

4.12 Gráfico para 2 cruzamentos em espaços amostrais finitos. . . . . . . . . . . . . . . 47

4.13 Gráfico do poder do testes para 2 cruzamentos, 2 populações e sem censura. . . . . . . . . 48

4.14 Gráfico do poder do testes para 2 cruzamentos, 2 populações e com censura. . . . . . . . . 49

4.15 Gráfico do poder do testes para 2 cruzamentos, 3 populações e sem censura. . . . . . . . . 50

4.16 Gráfico do poder do testes para 2 cruzamentos, 3 populações e com censura. . . . . . . . . 51

4.17 Gráfico da simulação sob a hipótese nula $H_{0}$ (sem censura) . . . . . . . . . . . . . . 53

4.18 Gráfico da simulação sob a hipótese nula $H_{0}$ (censura 90) . . . . . . . . . . . . . . . . 54

4.19 Gráfico da simulação sob a hipótese nula $H_{0}$ (censura 100) . . . . . . . . . . . . . . 55

4.20 Gráfico da simulação sob a hipótese nula $H_{0}$ (censura 105) . . . . . . . . . . . . . 56

4.21 Gráfico da simulação sob a hipótese nula $H_{0}$ (censura 110) . . . . . . . . . . . . . . 57

4.22 Gráficos da Função Poder $\lambda_{1}=\lambda=100$ e $\lambda_{2}=\lambda+\delta \ldots \ldots$. . . . . . . . . . 58

4.23 Cruzamento das funções de intensidade. . . . . . . . . . . . . . . . . . . 60

4.24 Função Poder para cruzamento das funções de intensidade. . . . . . . . . . . . . . . . . 61 
4.25 Gráfico da Simulação sob $H_{0}$ para vários tamanhos de amostra e $u\left(n^{\star}, \cdot\right)=1 \ldots$. . . . . 63

4.26 Gráfico do função poder para caso discreto $\left(W_{1} \sim \operatorname{Poi}(\lambda=1)\right.$ e $\left.W_{2} \sim \operatorname{Geo}(p=0,3)\right)$ sem censura. . . . . . . . . . . . . . . . . . . . . 64

4.27 Gráfico do função poder para caso discreto $\left(W_{1} \sim \operatorname{Poi}(\lambda=1)\right.$ e $\left.W_{2} \sim \operatorname{Geo}(p=0.3)\right)$ com censura 2 à direita. . . . . . . . . . . . . . . . . . . . 65

4.28 Gráfico do cenário geral para dados discretos. . . . . . . . . . . . . . . . . . . 66

4.29 Tratamento versus Controle para o caso 1 sem censura. . . . . . . . . . . . . . . . . 68

4.30 Tratamento versus Controle para o caso 1 com censura. . . . . . . . . . . . . . . . . 69

4.31 Tratamento versus Controle para o caso 2 sem censura. . . . . . . . . . . . . . . . 70

4.32 Tratamento versus Controle para o caso 2 com censura. . . . . . . . . . . . . . . . 71

4.33 Tratamento versus Controle para o caso 3 sem censura. . . . . . . . . . . . . . . . . . 72

4.34 Tratamento versus Controle para o caso 3 com censura. . . . . . . . . . . . . . . . . 73

4.35 Tratamento versus Controle para o caso 4 sem censura. . . . . . . . . . . . . . . . . . . 74

4.36 Tratamento versus Controle para o caso 4 com censura. . . . . . . . . . . . . . . . . . 75

4.37 Taxa de falha das distribuições Weibull e Exponencial para o $1^{\circ}$ experimento. . . . . . . . 77

4.38 Gráfico da Simulação sob $H_{0}$ para dados truncados e sem censura. . . . . . . . . . . . . 79

4.39 Gráfico da Simulação sob $H_{0}$ para dados truncados e com censura. . . . . . . . . . . . . 79

4.40 Gráfico da Simulação sob $H_{0}$ para dados truncados com 1 casa e sem censura. . . . . . . . 80

4.41 Gráfico da Simulação sob $H_{0}$ para dados truncados com 1 casa e com censura. . . . . . . . 80

4.42 Gráfico da Simulação sob $H_{0}$ para dados truncados com 2 casas e sem censura. . . . . . . 81

4.43 Gráfico da Simulação sob $H_{0}$ para dados truncados com 2 casas e com censura. . . . . . . 81

4.44 Gráfico para dados com truncamento completo na ausência de censura. . . . . . . . . . . 83

4.45 Gráfico para dados com truncamento completo na presença de censura. . . . . . . . . . . . 84

4.46 Gráfico para dados truncados com 1 casa na ausência de censura. . . . . . . . . . . . . . 85

4.47 Gráfico para dados truncados com 1 casa na presença de censura. . . . . . . . . . . . . 86

4.48 Gráfico para dados truncados com 2 casas na ausência de censura. . . . . . . . . . . . . 87

4.49 Gráfico para dados truncados com 2 casas na presença de censura. . . . . . . . . . . . 88

4.50 Taxa de falha das distribuições Weibull e Exponencial para o $2^{\circ}$ experimento. . . . . . . . 89

5.1 Estimativa para a função de sobrevivência e função de intensidade. . . . . . . . . . . . . . 95

5.2 Gráfico da funções de sobrevivência e e das funções de intensidade. . . . . . . . . . . . . . 98

5.3 Gráfico do cruzamento das funções de intensidade. . . . . . . . . . . . . . . . . . . . 99

5.4 Gráfico das funções de intensidade por Área. . . . . . . . . . . . . . . . . . . . . . 100 


\section{Lista de Tabelas}

1.1 Conjunto de dados do estudo de tumores gastrointestinais. O símbolo + significa que o dado

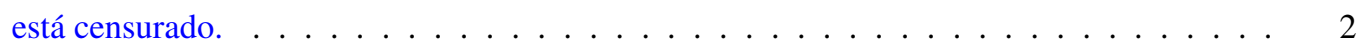

1.2 Alunos ingressante entre 2000 e 2003, acompanhados por 9 semestres. . . . . . . . . . . 3

1.3 Evasão por semestre. . . . . . . . . . . . . . . . . . . . . . 4

3.1 Funções de ponderação. . . . . . . . . . . . . . . . . . . . . . . . . 18

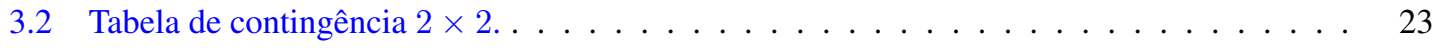

3.3 Resumo das observações para a $\ell$-ésima categoria. . . . . . . . . . . . . . . . . . 24

4.1 Simulação sob a hipótese nula $H_{0}$ para espaços finitos. . . . . . . . . . . . . . . . 33

4.2 Função Poder do teste para 2 populações, tamanho de amostra 500 e sem censura. . . . . . 35

4.3 Função Poder do teste para 3 populações, tamanho de amostra 500 e sem censura. . . . . . 36

4.4 Função Poder do teste para 5 populações, tamanho de amostra 500 e sem censura. . . . . . 37

4.5 Função Poder do teste para 2 populações, $\delta$ de 0,05 e sem censura. . . . . . . . . . . . . 39

4.6 Função Poder do teste para 3 populações, $\delta$ de 0,05 e sem censura. . . . . . . . . . . . . 40

4.7 Função Poder do teste para 5 populações, $\delta$ de 0,05 e sem censura. . . . . . . . . . . . 41

4.8 Função Poder do teste para 2 populações e sem censura. . . . . . . . . . . . . . . . . . 43

4.9 Função Poder do teste para 3 populações e sem censura. . . . . . . . . . . . . . . . . . . 44

4.10 Função Poder do teste para 5 populações e sem censura. . . . . . . . . . . . . . . . . . 45

4.11 Funções de intensidade para experimentos de 2 cruzamentos. . . . . . . . . . . . . . . 47

4.12 Tabela da função poder do testes para 2 cruzamentos, 2 populações e sem censura. . . . . . 47

4.13 Tabela da função poder do testes para 2 cruzamentos, 2 populações e com censura. . . . . 48

4.14 Tabela da função poder do testes para 2 cruzamentos, 3 populações e sem censura. . . . . . 49

4.15 Tabela da função poder do testes para 2 cruzamentos, 3 populações e com censura. . . . . 50

4.16 Avaliação do Erro do Tipo I para riscos proporcionais (Sem Censura) . . . . . . . . . . . . 52

4.17 Avaliação do Erro do Tipo I para riscos proporcionais (Censura 90) . . . . . . . . . . . . 53

4.18 Avaliação do Erro do Tipo I para riscos proporcionais (Censura 100) . . . . . . . . . . . . 54

4.19 Avaliação do Erro do Tipo I para riscos proporcionais (Censura 105) . . . . . . . . . . . . 55

4.20 Avaliação do Erro do Tipo I para riscos proporcionais (Censura 110) . . . . . . . . . . . . 56 
4.21 Avaliação da função Poder do Teste para riscos proporcionais . . . . . . . . . . . . . . 58

4.22 Função Poder do Teste para cruzamento das funções de intensidade . . . . . . . . . . . 61

4.23 Simulação sob $H_{0}$ para vários tamanhos de amostra . . . . . . . . . . . . . . . . . . 62

4.24 Tabela da função poder para caso discreto $\left(W_{1} \sim \operatorname{Poi}(\lambda=1)\right.$ e $\left.W_{2} \sim \operatorname{Geo}(p=0,3)\right)$ sem

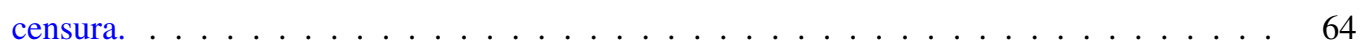

4.25 Tabela da função poder para caso discreto $\left(W_{1} \sim \operatorname{Poi}(\lambda=1)\right.$ e $\left.W_{2} \sim \operatorname{Geo}(p=0.3)\right)$ com censura 2 à direita. . . . . . . . . . . . . . . . . . . . . 65

4.26 Tratamento versus Controle para o caso 1 sem censura . . . . . . . . . . . . . . . 68

4.27 Tratamento versus Controle para o caso 1 com censura . . . . . . . . . . . . . . . . 69

4.28 Tratamento versus Controle para o caso 2 sem censura . . . . . . . . . . . . . . . . 70

4.29 Tratamento versus Controle para o caso 2 com censura . . . . . . . . . . . . . . . . 71

4.30 Tratamento versus Controle para o caso 3 sem censura . . . . . . . . . . . . . . . . 72

4.31 Tratamento versus Controle para o caso 3 com censura . . . . . . . . . . . . . . . . 73

4.32 Tratamento versus Controle para o caso 4 sem censura . . . . . . . . . . . . . . . . . 74

4.33 Tratamento versus Controle para o caso 4 com censura . . . . . . . . . . . . . . . . 75

4.34 Resultado sob $H_{0}$ para dados truncados e contínuos. . . . . . . . . . . . . . . . . 78

4.35 Dados com truncamento completo na ausência de censura. . . . . . . . . . . . . . . 83

4.36 Dados com truncamento completo na presença de censura. . . . . . . . . . . . . . . . 84

4.37 Dados truncados com 1 casa na ausência de censura. . . . . . . . . . . . . . . . 85

4.38 Dados truncados com 1 casa na presença de censura. . . . . . . . . . . . . . . 86

4.39 Dados truncados com 2 casas na ausência de censura . . . . . . . . . . . . . . . . . 87

4.40 Dados truncados com 2 casas na presença de censura. . . . . . . . . . . . . . . . 88

4.41 Resultados sob $H_{0}$ para o $2^{\circ}$ experimento. . . . . . . . . . . . . . . . . . . 90

4.42 Resultados para cruzamento das funções de intensidade na ausência e presença de censura

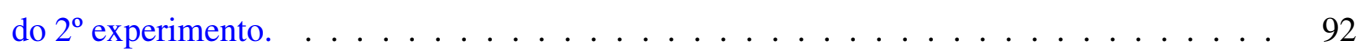

5.1 Aplicação do livro de Klein e Moeschberger (1997)[15] páginas 18 e 19. . . . . . . . . . . 95

5.2 Resumo com as Estatísticas e p-valores para diversos testes estatísticos. . . . . . . . . . 95

5.3 Estimativas dos parâmetros para o modelo Weibull. . . . . . . . . . . . . . . . . . 96

5.4 Status dos alunos. . . . . . . . . . . . . . . . . . . . 96

5.5 Evasão dos alunos de graduação por semestre . . . . . . . . . . . . . . . . . . . . . . 97

5.6 Comparação entre os anos de 2000 a 2003. . . . . . . . . . . . . . . . . . . . . . . . . 97

5.7 Comparação 2 a 2 entre os anos de 2000 a 2003 . . . . . . . . . . . . . . . . . . . . . 98

5.8 Comparação entre Áreas. . . . . . . . . . . . . . . . . . . . . . . . . . . . . . . . . . . 99

5.9 Resumo estatístico para comparação entre as áreas. . . . . . . . . . . . . . . . . . . 100

5.10 Comparação 2 a 2 entre as áreas Biológicas, Exatas e Humanas. . . . . . . . . . . . . . 100 


\section{Sumário}

1 Introdução $\quad 1$

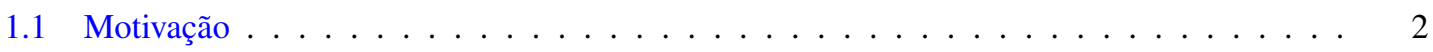

1.2 Objetivos e Organização do Trabalho . . . . . . . . . . . . . . . . . . . . 6

2 Análise de Dados Discretos na presença de Censura $\quad 7$

2.1 Inferência . . . . . . . . . . . . . . . . . . . . . 12

3 Comparação de dados discretos na presença de censura $\quad 16$

3.1 A estatística de Log-rank Ponderado . . . . . . . . . . . . . . . . . . . . 16

3.2 A estatística de Log-rank Modificado . . . . . . . . . . . . . . . . . . . . . 21

3.3 A estatística de Cramér-von Mises . . . . . . . . . . . . . . . . . 25

4 Estudo de Simulação $r$

4.1 Simulação para espaços amostrais finitos . . . . . . . . . . . . . . . 32

4.1 .1 Simulação sob a hipótese nula . . . . . . . . . . . . . . . . 32

4.1.2 Simulação do poder do teste para funções de intensidade proporcionais . . . . . . 34

4.1.3 Simulação do poder com 1 cruzamento nas funções intensidade . . . . . . . . . 42

4.1.4 Simulação do poder do teste com 2 cruzamentos das funções de intensidade . . . . 46

4.2 Espaços Amostrais Infinitos Enumeráveis . . . . . . . . . . . . . . . . . 51

4.2.1 Simulação para riscos proporcionais . . . . . . . . . . . . . . 51

4.2.2 Simulação com cruzamento das funções de intensidade . . . . . . . . . . . . 59

4.2.3 Comparação das funções de ponderação para cruzamento das funções de intensidade 60

4.3 Cenário geral para dados discretos . . . . . . . . . . . . . . . . . 65

4.4 Simulação em populações para dados truncados . . . . . . . . . . . . . . . . 76

5 Aplicações $\quad 94$

5.1 Aplicação para grupo de tumores gastrointestinais . . . . . . . . . . . . . . . . . . . . 94

5.2 Aplicação para Evasão de alunos de graduação da USP . . . . . . . . . . . . . . . . . . 96

6 Conclusões $\quad 102$ 
A.1 Parametrizações das distribuição de probabilidades utilizadas . . . . . . . . . . . . . . . 106 


\section{Capítulo 1}

\section{Introdução}

O teste de Log-rank foi proposto por Mantel-Haennszel (1959)[17] para comparar duas populações. Este teste foi estendido por Aalen (1978)[1] e Gill (1980)[7] para comparar duas curvas de sobrevivência sujeitas a dados censurados. O caso de $J$ populações com diversas estruturas de censura foi desenvolvido por Andersen et al (1982)[3].

Esse teste não paramétrico baseado na diferença entre as taxas de falha (ou funções intensidade) de cada curva de sobrevivência. Apesar do teste de Log-rank ser o teste não paramétrico mais utilizado para se comparar duas ou mais populações sujeitas a dados censurados, este teste apresentada duas restrições. Primeiro, toda a teoria assintótica envolvida com o teste de Log-rank, tem como hipótese o fato das populações envolvidas terem distribuições contínuas ou no máximo mistas. Segundo, o teste de Log-rank não apresenta bom comportamento quando as funções intensidade cruzam.

Os teoremas gerais propostos por Gill (1980)[7] e Andersen et al (1982)[3] não são aplicáveis ao caso discreto. O caso puramente discreto foi recentemente tratado por Leão e Ohashi (2011)[16]. Segundo [16], o teste de Log-rank é consistente apenas para o caso em que não ocorrem cruzamentos entre as funções intensidade. Klein e Moeschberger (1997)[15] mostraram que o teste de Log-rank tem baixo poder em situações no qual as funções intensidade cruzam. A principal causa do baixo poder neste tipo de situação, é que as diferenças iniciais em favor de uma população (antes do cruzamento) são canceladas após o cruzamento pelas diferenças em favor da outra população. Para exemplificar esta situação podemos citar o exemplo descrito na seção (1.1).

No caso de duas populações, diversas soluções foram avaliadas em Klein e Moeschberger (1997)[15], entre elas, citamos a classe de testes do tipo Renyi, proposta por Gill (1980)[7]. Entretanto, estes testes são aplicados apenas no caso de duas populações e para populações com distribuições contínuas. Baseado no clássico teste de Cramér von-Mises, Leão e Ohashi (2011)[16] propuseram modificações no teste de Log-rank de tal forma que este é consistente para qualquer hipótese alternativa.

O propósito desta dissertação são dois, primeiro, estudamos através de simulação Monte Carlo o desempenho dos testes de hipóteses do tipo Log-rank baseados nos trabalhos de Leão e Ohashi [16] aplicado a 
populações puramente discretas e populações contínuas observadas de forma truncada. Segundo, propomos uma modificação no teste de Log-rank para dados com espaços amostrais finitos, que generaliza o teste qui-quadrado na presença de censura .

\subsection{Motivação}

A motivação inicial é uma aplicação descrita em Klein e Moeschberger (1997)[15] no qual comparamos duas funções de sobrevivência na presença de cruzamento das estimativas de Kaplan-Meier. O Grupo de Estudo de Tumores Gastrointestinais (1982) reportou os resultados do estudo comparando a quimioterapia versus quimioterapia combinada com a radioterapia no tratamento de um câncer gástrico. O tempo de sobrevivência reportado em dias, para os 45 pacientes em cada tratamento são os seguintes:

\begin{tabular}{|c|c|c|c|c|c|c|c|c|}
\hline \multicolumn{9}{|c|}{ Quimioterapia } \\
\hline 1 & 63 & 105 & 129 & 182 & 216 & 250 & 262 & 301 \\
\hline 301 & 342 & 354 & 356 & 358 & 380 & 383 & 383 & 388 \\
\hline 394 & 408 & 460 & 489 & 499 & 523 & 524 & 535 & 562 \\
\hline 569 & 675 & 676 & 748 & 778 & 786 & 797 & 955 & 968 \\
\hline 1000 & 1245 & 1271 & 1420 & 1551 & 1694 & 2363 & $2754+$ & $2950+$ \\
\hline \multicolumn{9}{|c|}{ Quimioterapia combinada com a Radioterapia } \\
\hline 17 & 42 & 44 & 48 & 60 & 72 & 74 & 95 & 103 \\
\hline 108 & 122 & 144 & 167 & 170 & 183 & 185 & 193 & 195 \\
\hline 197 & 208 & 234 & 235 & 254 & 307 & 315 & 401 & 445 \\
\hline 464 & 484 & 528 & 542 & 547 & 577 & 580 & 795 & 855 \\
\hline 1366 & 1577 & 2060 & $2412+$ & $2486+$ & $2796+$ & $2802+$ & $2934+$ & $2988+$ \\
\hline
\end{tabular}

Tabela 1.1: Conjunto de dados do estudo de tumores gastrointestinais. O símbolo + significa que o dado está censurado.

A principal motivação destes dados é o cruzamento das funções de intensidades empíricas. Nesta situação, a maioria dos testes têm baixo poder (ver Klein e Moeschberger (1997) [15]), fato que é um dos principais problemas em testes de hipóteses para a homogeneidade de curvas de sobrevivência. A figura (1.1) ilustra o cruzamento entre as estimativas de Kaplan-Meier. 


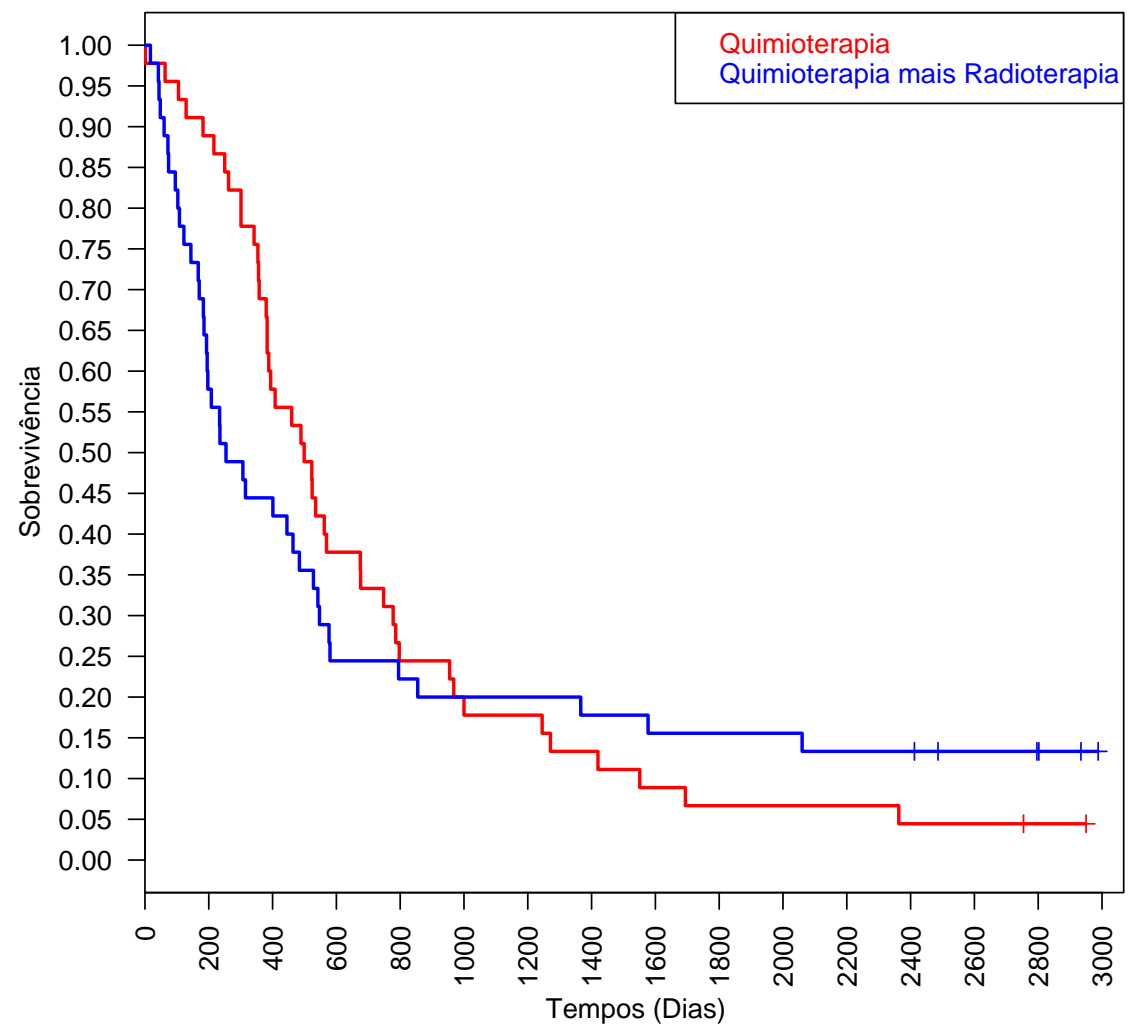

Figura 1.1: Produto limite estimado da distribuição da sobrevivência.

Outra aplicação interessante, foi extraída do trabalho de Pelissari (2009)[18] referente aos dados dos alunos ingressantes por vestibular no nível de graduação da Universidade de São Paulo - USP (Fonte: Sistema Júpter Web). Neste estudo, o principal objetivo é avaliar o tempo até a evasão dos alunos ingressantes por vestibular entre os anos de 1999 e 2008. Para que possamos avaliar a evolução da evasão ao longo dos anos, consideramos apenas os alunos que ingressaram entre de 2000 a 2003, sendo estes alunos acompanhados por 9 semestres.

\begin{tabular}{ccc}
\hline Status do aluno & Número de Alunos & Porcentagem \\
\hline Em Andamento & 6602 & $21,18 \%$ \\
Conclusão & 18305 & $58,71 \%$ \\
Evasão & 4913 & $15,76 \%$ \\
Falecimento & 39 & $0,12 \%$ \\
Migração & 1317 & $4,23 \%$ \\
Total & 31176 & $100 \%$ \\
& & \\
\hline
\end{tabular}

Tabela 1.2: Alunos ingressante entre 2000 e 2003, acompanhados por 9 semestres. 
O termo evasão foi definido pela Pró-Reitoria de graduação da USP é referido ao aluno que se desligou da USP a seu pedido ou por motivos administrativos ( 3 semestres sem matrícula, jubilamento ${ }^{1}$ ). Enquanto o termo migração é referido ao aluno que mudou de curso dentro da USP. De acordo com a tabela (5.4), concluímos que $21,18 \%$ dos alunos estão com o curso em andamento, 58,71\% concluíram e 15,76\% evadiram ao longo de 9 semestres de acompanhamento.

\begin{tabular}{|c|c|c|c|c|c|c|c|c|c|c|c|c|}
\hline Ano & Semestre & Evadiu & Não Evadiu & Sob risco & Semestre & Evadiu & Não Evadiu & Sob risco & Semestre & Evadiu & Não Evadiu & Sob risco \\
\hline 2000 & 1 & 285 & 874 & 1159 & 2 & 257 & 617 & 874 & 3 & 117 & 500 & 617 \\
\hline 2001 & & 179 & 798 & 977 & & 206 & 592 & 798 & & 105 & 487 & 592 \\
\hline 2002 & & 179 & 903 & 1082 & & 297 & 606 & 903 & & 125 & 481 & 606 \\
\hline 2003 & & 212 & 830 & 1042 & & 228 & 602 & 830 & & 117 & 485 & 602 \\
\hline Total & & 855 & 3405 & 4260 & & 988 & 2417 & 3405 & & 464 & 1953 & 2417 \\
\hline 2000 & 4 & 110 & 390 & 500 & 5 & 118 & 272 & 390 & 6 & 94 & 178 & 272 \\
\hline 2001 & & 101 & 386 & 487 & & 108 & 278 & 386 & & 86 & 192 & 278 \\
\hline 2002 & & 92 & 389 & 481 & & 103 & 286 & 389 & & 67 & 219 & 286 \\
\hline 2003 & & 104 & 381 & 485 & & 100 & 281 & 381 & & 60 & 221 & 281 \\
\hline Total & & 407 & 1546 & 1953 & & 429 & 1117 & 1546 & & 307 & 810 & 1117 \\
\hline 2000 & 7 & 68 & 110 & 178 & 8 & 47 & 63 & 110 & 9 & 63 & 0 & 63 \\
\hline 2001 & & 71 & 121 & 192 & & 59 & 62 & 121 & & 62 & 0 & 62 \\
\hline 2002 & & 81 & 138 & 219 & & 71 & 67 & 138 & & 67 & 0 & 67 \\
\hline 2003 & & 109 & 112 & 221 & & 73 & 39 & 112 & & 39 & 0 & 39 \\
\hline Total & & 329 & 481 & 810 & & 250 & 231 & 481 & & 231 & 0 & 231 \\
\hline
\end{tabular}

Tabela 1.3: Evasão por semestre.

Alunos de graduação da Universidade de São Paulo

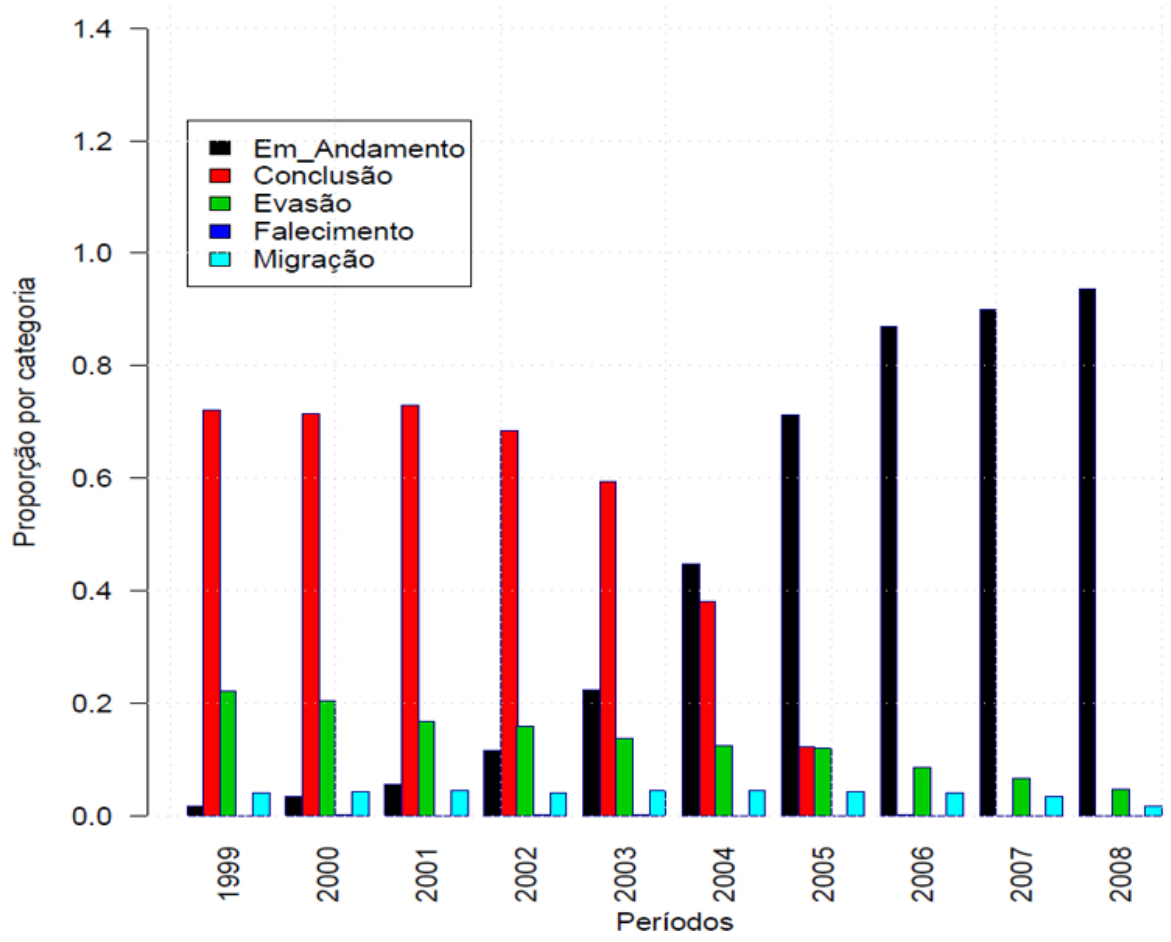

Figura 1.2: Gráfico dos alunos em cada uma das classes desde a última ocorrência.

\footnotetext{
${ }^{1}$ Este é um termo usado quando um aluno é desligado por não ter atingido desempenho satisfatório na universidade de acordo com critérios da mesma.
} 
Notamos pela figura (1.2), que a proporção de alunos que concluíram o curso vem diminuindo, pois à partir do ano de 2005 têm muitos alunos que não atingiram o tempo necessário para conclusão do curso, ao contrário da proporção dos alunos em andamento que vem aumentando pelo mesmo motivo dito anteriormente. Razão essa de tomarmos o intervalo de 2000 a 2003 com nove semestres para cada grupo. O intuito desta aplicação é na avaliação do cruzamento severo das funções de intensidade, fato visto na figura (1.3), entre os anos de 2000 e 2003.

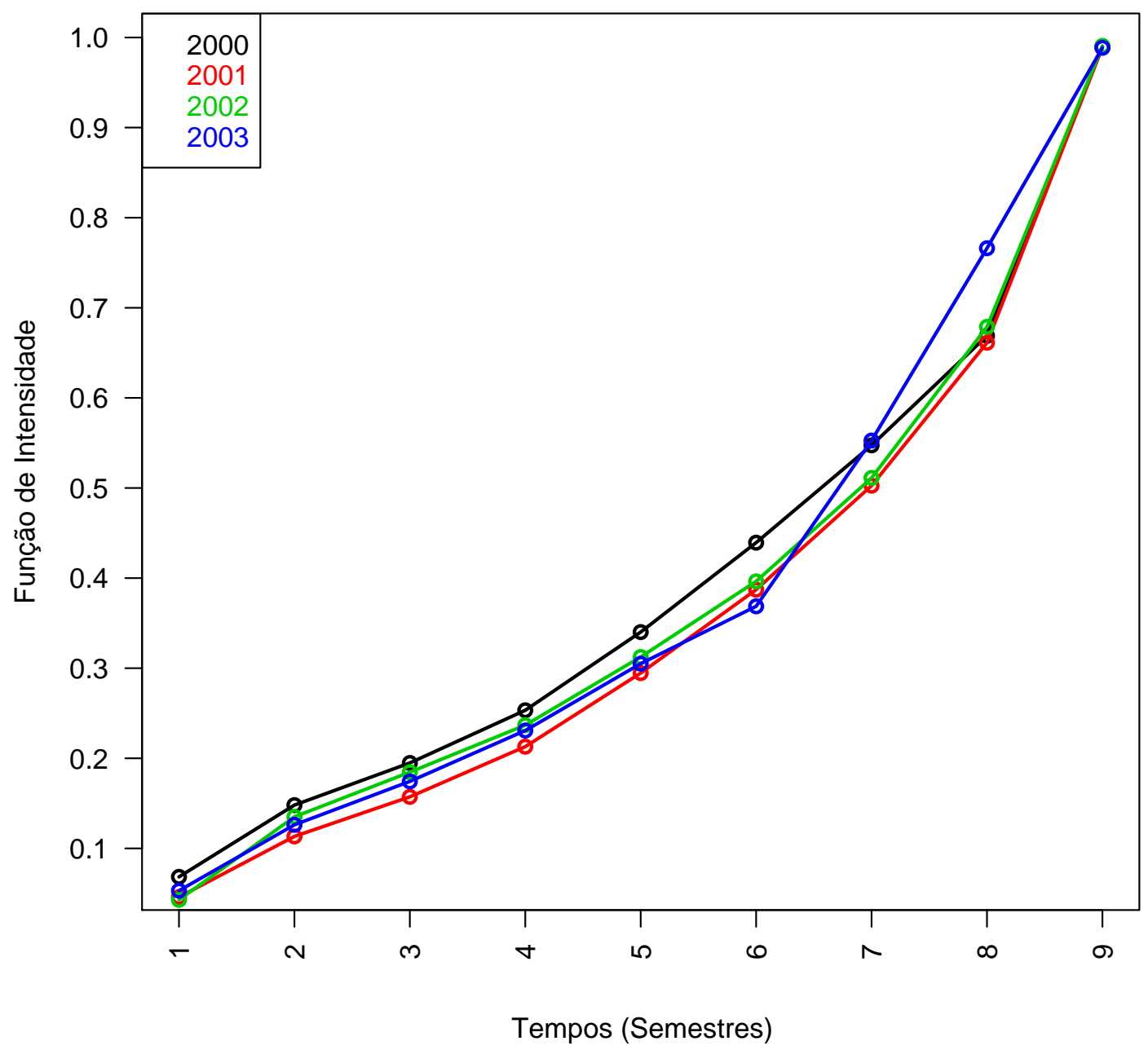

Figura 1.3: Função de Intensidade para o tempo até a evasão dos alunos ingressantes por vestibular. 


\subsection{Objetivos e Organização do Trabalho}

O principal objetivo do nosso trabalho é o tratamento de dados com taxa de falha não proporcional e o impacto da discretização dos dados na função poder dos testes usuais. Baseados no trabalho de Leão e Ohashi [16], avaliamos a estatística de Cramér-von Mises, que nos permite detectar diferenças significativas para riscos não proporcionais sujeitas a censuras arbitrárias para dados discretos ou arredondados. Além disso, propomos uma modificação do teste de Log-rank para espaços amostrais finitos, que aplicamos principalmente à tabelas de contingência. Ao aplicarmos estas estatísticas para dados discretos ou arredondados, o teste enunciado neste trabalho apresenta uma função poder maior do que os testes usuais. A seguir, resumimos a organização do trabalho da seguinte forma.

No segundo capítulo, introduzimos ao modelo não-paramétrico para dados discretos na presença de censura arbitrária. Neste capítulo, também mostramos alguns exemplos de funções de intensidade (ou taxa de falha) para os modelos discretos.

No terceiro capítulo, definimos as estatísticas estudas neste trabalho baseadas no trabalho de Leão e Ohashi [16] e uma modificação no teste de Log-rank. Neste capítulo, apresentamos o algoritmo dos testes para um melhor entendimento das notações utilizadas neste trabalho.

O quarto capítulo foi dedicado ao estudo de simulação, em que estudamos as estatísticas em várias situações, como por exemplo, para espaços amostrais finitos e infinitos enumeráveis. Dentro deste último espaço amostral, estudamos os casos em que os riscos são proporcionais, riscos não proporcionais (cruzamento das funções de intensidade) em dados discretos. Na sequência, estudamos o cenário geral com todas as situações possíveis, como por exemplo, sob a hipótese nula, cruzamento das funções de intensidade, proporcionais, não proporcionais porém sem cruzamento das funções de intensidade. Por fim, no último estudo avaliamos os testes em dados truncados.

No capítulo seguinte, aplicamos os testes a dois conjuntos de dados. Primeiro, avaliamos o clássico conjunto de dados referente ao grupo de estudos de tumores gastrointestinais (ver, Klein e Moeschberger (1997)[15]). Segundo, estudamos o número de semestres até a evasão de alunos de graduação da Universidade de São Paulo (USP) (ver, Pelissari (2009)[18]).

Finalizamos a dissertação com uma breve conclusão e considerações finais. 


\section{Capítulo 2}

\section{Análise de Dados Discretos na presença}

\section{de Censura}

Dados discretos surgem em diversas situações comuns em confiabilidade, economia, manutenção, ciências sociais entre outros. Um típico exemplo de dados discretos em economia do trabalho está relacionado à duração do desemprego medidos em meses completos de desemprego que é um exemplo em que a resposta é um valor inteiro. Uma ampla discussão sobre as consequências de modelos discretos para dados usados em economia com distribuição de probabilidade contínua é apresentada por Grimshaw et al (2005)[8]. Outra aplicação para dados discretos, podemos citar os índices de "evasão da Universidade”, em que o tempo para concluir o curso é medida em "semestres”. Em aplicações industriais, alguns equipamentos operam sob demanda ou em ciclos, e nestes casos, contamos o número de operações ou ciclos até a falha. Assim, embora tenhamos uma abundância de estudos na literatura para dados relativos a tempo de sobrevivência contínuos, ver Aalen (1978)[1], Fleming e Harrington (1990)[5], Andersen et al. (1993)[3] e Aalen et al (2009)[2], há uma escassez de estudos na literatura aplicados à dados relativos a tempo de sobrevivência discreto, ver Gupta et al (1997)[9], Grimshaw et al (2005)[8], Karlis e Patilea (2007)[14] e Yu (2007)[22], tendo em vista isto, vamos apresentar o modelo de intensidade para variáveis aleatórias discretas na presença de censura baseados nos trabalhos de Leão e Ohashi [16].

Consideramos $(\Omega, \mathcal{F}, P)$ um espaço de probabilidade e seja $\mathcal{K}=\{1, \ldots, k\} \subseteq \mathbb{N}$ finito ou não. Seja $Y$ uma variável aleatória com valores em $\mathcal{K}$ sendo $\pi_{i}=P[Y=i]$ a probabilidade da variável resposta $(Y)$ assumir a $i$-ésima categoria. Assumindo que as categorias são mutuamente exclusivas, temos que $\sum_{i=1}^{k} \pi_{i}=1$. Neste caso, dizemos que $Y$ é uma variável aleatória discreta.

Consideramos $W$ e $C$ duas variáveis aleatórias independentes discretas com valores em $\mathcal{K}$. A variável aleatória discreta $W$ descreve o evento de interesse, enquanto a variável aleatória discreta $C$ denota a 
variável de censura. Seja $X$ a variável aleatória definida por

$$
X=W \wedge C:=\min \{W, C\}
$$

Baseados em $X$ definimos os processos de contagem

$$
R(i):=\mathbb{1}_{\{X \leq i, X=W\}} \quad \text { e } \quad R^{C}(i):=\mathbb{1}_{\{X \leq i, X=C\}}, \quad i=0,1, \ldots, k .
$$

O processo de contagem $R\left(R^{C}\right)$ determina a categoria para o qual o evento de interesse (censura) ocorre. Tomamos $(\Omega, \mathcal{F}, \mathbf{A}, P)$ a base estocástica com filtragem gerada por $R$ e $R^{C}$, como a seguir

$$
\mathcal{A}_{0}:=\{\Omega, \emptyset\}, \quad \mathcal{A}_{i}:=\sigma\left\{\Delta R(\ell), \Delta R^{C}(\ell): 1 \leq \ell \leq i\right\}, \quad i \in \mathcal{K},
$$

no qual, $\mathbf{A}=\left\{\mathcal{A}_{i} ; i=0,1, \ldots, k\right\}$. Obtemos que $R$ é um processo $\mathbf{A}$-adaptado, não decrescente e consequentemente, obtemos da decomposição de Doob-Meyer que $R=Y+A$, no qual $Y$ é um martingale e $A$ é um processo previsível não decrescente satisfazendo

$$
Y(i)=\sum_{\ell=1}^{i}\left(R(\ell)-E\left[R(\ell) \mid \mathcal{A}_{\ell-1}\right]\right) \quad \text { e } A(i)=\sum_{\ell=1}^{i}\left(E\left[R(\ell) \mid \mathcal{A}_{\ell-1}\right]-R(\ell-1)\right),
$$

com $A(0)=0, Y(0)=0$. Calculando os processos estocásticos relacionados com a decomposição.

$$
\begin{aligned}
A(1) & =E\left[R(1) \mid \mathcal{A}_{0}\right]-R(0)=E[R(1)]=E\left[\mathbb{1}_{\{X \leq 1, X=W\}}\right]=P[W=1] \\
A(2) & =A(1)+E\left[R(2) \mid \mathcal{A}_{1}\right]-R(1)=P[W=1]+E\left[R(2)-R(1) \mid \mathcal{A}_{1}\right]= \\
& =P[W=1]+E[R(2)-R(1) \mid R(1), C]
\end{aligned}
$$

Notamos que, se $R(1)=1$, então

$$
R(2)-R(1)=\mathbb{1}_{\{X=2, X=W\}}=0
$$

Disto, obtemos

$$
\begin{aligned}
& E[R(2)-R(1) \mid R(1), C]=0, \quad \text { se } R(1)=0 \text { e } C=1 \\
& E[R(2)-R(1) \mid R(1), C] \neq 0, \quad \text { se } R(1)=0 \text { e } C \geq 2
\end{aligned}
$$


Com, $W$ e $C$ independentes obtemos

$$
\begin{aligned}
E[R(2)-R(1) \mid R(1), C] & =E[R(2)-R(1) \mid R(1)=0, C \geq 2] \mathbb{1}_{\{R(1)=0, C \geq 2\}}= \\
& =E\left[\mathbb{1}_{\{X \leq 2, X=W\}}-\mathbb{1}_{\{X \leq 1, X=W\}} \mid R(1)=0, C \geq 2\right] \mathbb{1}_{\{R(1)=0, C \geq 2\}}= \\
& =E\left[\mathbb{1}_{\{W=2\}} \mid R(1)=0, C \geq 2\right] \mathbb{1}_{\{R(1)=0, C \geq 2\}}= \\
& =\frac{P[W=2, R(1)=0, C \geq 2]}{P[R(1)=0, C \geq 2]} \mathbb{1}_{\{R(1)=0, C \geq 2\}}= \\
& =\frac{P[W=2]}{P[W \geq 2]} \mathbb{1}_{\{R(1)=0, C \geq 2\}}
\end{aligned}
$$

Assim, substituindo em (2.2) obtemos

$$
A(2)=A(1)+\frac{P[W=2]}{P[W \geq 2]} \mathbb{1}_{\{R(1)=0, C \geq 2\}}
$$

Realizando analogamente os cálculos, obtemos

$$
A(k)=A(k-1)+\frac{P[W=k]}{P[W \geq k]} \mathbb{1}_{\{R(k-1)=0, C \geq k\}}
$$

De forma geral, obtemos

$$
A(i)=\sum_{\ell=1}^{i} \frac{P[W=\ell]}{P[W \geq \ell]} \mathbb{1}_{\left\{R(\ell-1)=0, R^{C}(\ell-1)=0\right\}}=\sum_{\ell=1}^{i} \frac{P[W=\ell]}{P[W \geq \ell]} \mathbb{1}_{\{X \geq \ell\}} \text { q.c. }
$$

para qualquer $i \geq 1$.

Portanto, a função de intensidade $h:\{0,1, \cdots, k\} \rightarrow[0,1]$ associado ao processo de contagem $R$ é dada por

$$
h(i):=\frac{P[W=i]}{P[W \geq i]}
$$

com $h(0)=0,0<h(i)<1$, para qualquer $i=1, \ldots, k$ e $h(k)=1$ (caso $k$ seja finito). Com isso, obtemos que $h$ descreve unicamente a distribuição de probabilidade da variável aleatória $W$ (que descreve o evento de interesse). Além disso, temos que

$$
P[W=i]=h(i) \prod_{\ell=1}^{i-1}[1-h(\ell)]
$$

De fato,

$$
P[W \geq i-1]=\prod_{\ell=1}^{i-2}(1-h(\ell)), \quad \text { hipótese de indução }
$$


Por indução sobre $i$, obtemos que

$$
\begin{aligned}
P[W \geq i] & =1-\sum_{\ell=1}^{i-1} P[W=\ell]=1-\sum_{\ell=1}^{i-2} P[W=\ell]-P[W=i-1]= \\
& \stackrel{2.6}{=} \prod_{\ell=1}^{i-2}(1-h(\ell))-P[W=i-1]=\prod_{\ell=1}^{i-2}(1-h(\ell))-h(i-1) P[W \geq i-1]= \\
& =\prod_{\ell=1}^{i-2}(1-h(\ell))-h(i-1) \prod_{\ell=1}^{i-2}(1-h(\ell))= \\
& =\prod_{\ell=1}^{i-1}(1-h(\ell))
\end{aligned}
$$

Portanto

$$
P[W=i]=h(i) \prod_{\ell=1}^{i-1}(1-h(\ell))
$$

Assim, concluímos que a relação apresentada na equação (2.5) é válida. Agora, vamos exemplificar algumas funções de intensidade para dados discretos à partir de distribuições de probabilidade conhecidas.

\section{Exemplo 2.1. (Distribuição Geométrica)}

$P[W=i]=(1-p)^{i} p$ com parâmetro $p, 0<p<1$. Assim, obtemos pela definição a função de intensidade para a distribuição Geométrica da seguinte forma

$$
h(i):=\frac{P[W=i]}{P[W \geq i]}=\frac{(1-p)^{i} p}{1-p \underbrace{\sum_{\ell=0}^{i}(1-p)^{\ell}}_{\frac{(1-p)^{i}-1}{(1-p)-1}}}=\frac{(1-p)^{i} p}{(1-p)^{i}}=p
$$

que é uma taxa de falha constante.

\section{Exemplo 2.2. (Distribuição de Poisson)}

$P[W=i]=\frac{e^{-\lambda} \lambda^{i}}{i !}, i=1,2, \ldots, \lambda>0$. Assim, obtemos pela definição a função de intensidade para a distribuição Poisson da seguinte forma

$$
h(i):=\frac{P[W=i]}{P[W \geq i]}=\frac{\frac{e^{-\lambda} \lambda^{i}}{i !}}{1-\sum_{j=1}^{i-1} \frac{e^{-\lambda} \lambda^{j}}{j !}}=\frac{\frac{e^{-\lambda} \lambda^{i}}{i !}}{e^{-\lambda} \sum_{j=i}^{\infty} \frac{\lambda^{j}}{j !}}=\frac{\lambda^{i}}{i !}\left(\sum_{j=i}^{\infty} \frac{\lambda^{j}}{j !}\right)^{-1}
$$

\section{Exemplo 2.3. (Distribuição Binomial)}

$$
P[W=i]=\left(\begin{array}{l}
n \\
k
\end{array}\right) p^{i}(1-p)^{n-i}, \quad i=0,1,2, \ldots, n \text {. Assim, obtemos pela definição a função de }
$$
intensidade para a distribuição Binomial da seguinte forma 


$$
h(i):=\frac{P[W=i]}{P[W \geq i]}=\frac{\left(\begin{array}{c}
n \\
i
\end{array}\right) p^{i}(1-p)^{n-i}}{\sum_{j=i}^{n}\left(\begin{array}{c}
n \\
j
\end{array}\right) p^{j}(1-p)^{n-j}}=\left(\begin{array}{c}
n \\
i
\end{array}\right)\left(\sum_{j=i}^{n}\left(\begin{array}{c}
n \\
j
\end{array}\right)\left(\frac{p}{1-p}\right)^{j-i}\right)^{-1}
$$

\section{Exemplo 2.4. (Distribuição Binomial Negativa)}

$$
P[W=i]=\left(\begin{array}{c}
k+i-1 \\
i
\end{array}\right) p^{k}(1-p)^{i}, \quad 0<p<1, n>0, i=0,1,2, \ldots, n . \text { Assim, obtemos pela }
$$

definição a função de intensidade para a distribuição Binomial Negativa da seguinte forma

$h(i):=\frac{P[W=i]}{P[W \geq i]}=\frac{\left(\begin{array}{c}k+i-1 \\ i\end{array}\right) p^{k}(1-p)^{i}}{\sum_{j=i}^{n}\left(\begin{array}{c}k+j-1 \\ j\end{array}\right) p^{k}(1-p)^{j}}=\left(\begin{array}{c}k+i-1 \\ i\end{array}\right)\left(\sum_{j=i}^{n}\left(\begin{array}{c}k+j-1 \\ j\end{array}\right)(1-p)^{j-i}\right)^{-1}$

\section{Exemplo 2.5. (Distribuição Série Logarítmica)}

$P[W=i]=-\frac{\theta^{i}}{i \log (1-\theta)}, 0<\theta<1, i=1,2, \ldots$ Assim, obtemos pela definição a função de intensidade para a distribuição Série Logarítmica da seguinte forma

$$
h(i):=\frac{P[W=i]}{P[W \geq i]}=\frac{-\frac{\theta^{i}}{i \log (1-\theta)}}{-\sum_{j=i}^{\infty} \frac{\theta^{j}}{j \log (1-\theta)}}=\frac{\theta^{i}}{i}\left(\sum_{j=1}^{i-1} \frac{\theta^{j}}{j}-\log (1-\theta)\right)^{-1}
$$

Retornamos a caracterização do processo de contagem. Seja $H$ o processo de intensidade acumulado definido por $H(i):=\sum_{\ell=1}^{i} h(\ell)$ e $V(\ell):=\mathbb{1}_{\{X \geq \ell\}}$ o processo de risco, são dois processos adaptados à filtragem $\mathbf{A}=\left\{\mathcal{A}_{i} ; i=0,1, \ldots, k\right\}$.

$$
A(i)=(V . H)(i) \stackrel{2.3}{=} \sum_{\ell=1}^{i} h(\ell) \mathbb{1}_{\{X \geq \ell\}}=\sum_{\ell=1}^{i} V(\ell) \Delta H(i), \quad i=0,1, \ldots, k,
$$

Assim, temos que

$$
\begin{aligned}
R(i) & =Y(i)+A(i)= \\
& =\sum_{\ell=1}^{i}\left(R(\ell)-E\left[R(\ell) \mid \mathcal{A}_{\ell-1}\right]\right)+\sum_{\ell=1}^{i} V(\ell) \Delta H(i)= \\
& =\sum_{\ell=1}^{i}\left(R(\ell)-E\left[R(\ell) \mid \mathcal{A}_{\ell-1}\right]\right)+(V . H)(i), \quad i=0,1, \ldots, k,
\end{aligned}
$$

Além disso, temos que a representação $A(i)$ é um processo previsível representado na forma multiplicativa, no qual $\Delta H(\ell)=H(\ell)-H(\ell-1)=h(\ell)$. Como $V$ é previsível, o processo $A$ é determinado pela 
função intensidade $h$ (ou, pela função intensidade acumulada $H$ ). Desta forma, temos uma relação um-aum entre a função intensidade $h$ e a variável aleatória $W$ via relação (2.4). Portanto, para compararmos distribuições discretas vamos avaliar as funções intensidade envolvidas. O processo (2.7) é denominado modelo de intensidade multiplicativo (ver Aalen (1978)[1]) para variáveis aleatórias discretas. A seguir, apresentamos a inferência para o modelo discreto na presença de censura.

\subsection{Inferência}

Nesta seção vamos seguir Fleming e Harrington (1990) [5] para a definição do modelo discreto na presença de censura aleatória. Para isto, consideramos os pares ordenados $\left\{\left(W_{m}^{p}, C_{m}^{p}\right): m=1, \ldots, n_{p}, p=\right.$ $1, \ldots, J\}$ de variáveis aleatórias discretas, independentes e com a mesma distribuição do par de variáveis aleatórias discretas $\left(W^{p}, C^{p}\right), p=1, \ldots, J$. Algumas das variáveis aleatórias $W_{m}^{p}$ são observadas, mas o resto é apenas conhecido por ser maior do as variáveis aleatórias discretas $C_{m}^{p}$. Assumimos que $W^{p}$ e $C^{p}$ são variáveis aleatórias independentes. Assim, tomamos os processos de contagem

$$
R_{m}^{p}(i):=\mathbb{1}_{\left\{X_{m}^{p} \leq i, X_{m}^{p}=W_{m}^{p}\right\}} \quad \text { e } \quad R_{m}^{C, p}(i):=\mathbb{1}_{\left\{X_{m}^{p} \leq i, X_{m}^{p}=C_{m}^{p}\right\}}
$$

para qualquer $m=1, \ldots, n_{p}$ e $p=1, \ldots, J$. Portanto, o processo de contagem associado a $p$-ésima amostra aleatória é dado por

$$
R^{n_{p}}(i):=\sum_{m=1}^{n_{p}} R_{m}^{p}(i) \text { e } R_{m}^{C, n_{p}}(i):=\sum_{m=1}^{n_{p}} R_{m}^{C, p}(i), \quad i \in \mathcal{K} \text { e } p=1, \ldots, J
$$

Similarmente a equação (2.1), tomamos $\mathbb{F}^{n_{p}}:=\left\{\mathcal{F}_{i}^{n_{p}}, i=0,1, \ldots, k\right\}$ a filtragem definida por

$$
\mathcal{F}_{0}^{n_{p}}=\{\Omega, \emptyset\}, \quad \mathcal{F}_{i}^{n_{p}}:=\sigma\left(\Delta \mathcal{R}_{n_{p}}^{C, p}(\ell), \Delta \mathcal{R}_{n_{p}}^{p}(\ell) ; 1 \leq \ell \leq i\right)=\sigma\left\{\mathcal{A}_{1, i}^{p} \cup \cdots \cup \mathcal{A}_{n_{p}, i}^{p}\right\},
$$

no qual

$$
\mathcal{R}_{n_{p}}^{p}(i):=\left(R_{1}^{p}(i), \ldots, R_{n_{p}}^{p}(i)\right), \quad \mathcal{R}_{n_{p}}^{C, p}(i):=\left(R_{1}^{C, p}(i), \ldots, R_{n_{p}}^{C, p}(i)\right)
$$

e

$$
\mathcal{A}_{m, i}^{p}:=\sigma\left(\Delta R_{m}^{C, p}(\ell), \Delta R_{m}^{p}(\ell) ; 1 \leq \ell \leq i\right), \quad \text { para } i=0,1, \ldots, k \text { e } m=1, \ldots, n_{p}
$$

Finalmente, denotamos por $\mathbb{F}$ a filtragem gerada pelas filtragens $\left\{\mathbb{F}^{n_{p}}: n_{p} \in \mathbb{N}, p=1, \cdots, J\right\}$, que corresponde ao total de informação existente nas amostras. Através da decomposição de Doob-Meyer para $\left(R^{n_{p}}, \mathbf{F}^{n_{p}}\right)$, obtemos da equação (2.8) que 


$$
\begin{aligned}
R^{n_{p}}(i) & =\sum_{\ell=1}^{i}\left(R^{n_{p}}(\ell)-\mathbf{E}\left[R^{n_{p}}(\ell) \mid \mathcal{F}_{\ell-1}^{n_{p}}\right]\right)+\sum_{\ell=1}^{i}\left(\mathbf{E}\left[R^{n_{p}}(\ell) \mid \mathcal{F}_{\ell-1}^{n_{p}}\right]-R^{n_{p}}(\ell-1)\right)= \\
& =\sum_{\ell=1}^{i}\left(\sum_{m=1}^{n_{p}} R_{m}^{p}(\ell)-\mathbf{E}\left[\sum_{m=1}^{n_{p}} R_{m}^{p}(\ell) \mid \mathcal{F}_{\ell-1}^{n_{p}}\right]\right)+A^{n_{p}}(i)= \\
& =\sum_{m=1}^{n_{p}}\left(\sum_{\ell=1}^{i} R_{m}^{p}(\ell)-\mathbf{E}\left[\sum_{\ell=1}^{i} R_{m}^{p}(\ell) \mid \mathcal{F}_{\ell-1}^{n_{p}}\right]\right)+A^{n_{p}}(i)= \\
& =\sum_{m=1}^{n_{p}}\left(\sum_{\ell=1}^{i} R_{m}^{p}(\ell)-\mathbf{E}\left[\sum_{\ell=1}^{i} R_{m}^{p}(\ell) \mid \mathcal{A}_{m, \ell-1}^{p}\right]\right)+A^{n_{p}}(i)= \\
& =\sum_{m=1}^{n_{p}} Y_{m}^{p}(i)+A^{n_{p}}(i)=Y^{n_{p}}(i)+A^{n_{p}}(i)
\end{aligned}
$$

no qual, $R_{m}^{p}(i)=Y_{m}^{p}(i)+A_{m}^{p}(i)$ para qualquer $i=0,1, \ldots, k$ e $p=1, \cdots, J$. Assim, obtemos que $Y^{n_{p}}$ é um $\mathbf{F}^{n_{p}}$-martingale e $A^{n_{p}}$ é um processo previsível não decrescente, tal que $A^{n_{p}}(i)=\sum_{m=1}^{n_{p}} A_{m}^{p}(i)$. Além disso, o processo de risco associado é dado por

$$
V^{n_{p}}(i)=\sum_{m=1}^{n_{p}} V_{m}^{p}(i), \quad \text { em que } V_{m}^{p}(i)=\mathbb{1}_{\left\{X_{m}^{p} \geq i\right\}}
$$

com

$$
A_{m}^{p}(i)=\left(V_{m}^{p} \cdot H^{p}\right)(i), \quad A^{p}(i)=\left(V^{p} \cdot H^{p}\right)(i), \quad \pi_{i}^{p}=\mathbf{P}\left[W^{p}=i\right]=h^{p}(i) \prod_{\ell=1}^{i-1}\left[1-h^{p}(\ell)\right]
$$

$\mathrm{e}$

$$
h^{p}(j):=\frac{\mathbf{P}\left[W^{p}=j\right]}{\mathbf{P}\left[W^{p} \geq j\right]}, \quad H^{p}(i):=\sum_{j=1}^{i} h^{p}(j), \quad i=1,2,3, \cdots, k \quad \text { e } \quad p=1, \cdots, J .
$$

Para mais detalhes sobre o modelo de intensidade multiplicativo para variáveis aleatórias discretas, ver Pelissari (2009)[18]. Através de argumentos clássicos da teoria de martingale (Fleming e Harrington (1990))[5], obtemos que um estimador de $h^{p}$ é dado pelo estimador de Kaplan-Meier igualando o martingale $Y^{n_{p}}$ a zero. De fato, para $i=1$ temos

$$
Y^{n_{p}}(1)=R^{n_{p}}(1)-\hat{h}^{n_{p}}(1) \sum_{m=1}^{n_{p}} \mathbb{1}_{\left\{X_{m}^{p} \geq 1\right\}}=0 \quad \Rightarrow \quad \hat{h}^{n_{p}}(1)=\frac{R^{n_{p}}(1)}{V^{n_{p}}(1)} \mathbb{1}_{\left\{V^{n_{p}}(1)>0\right\}}
$$


Agora, para $i=2$ temos

$$
\begin{aligned}
& Y^{n_{p}}(2)=R^{n_{p}}(2)-\left[\hat{h}^{n_{p}}(2) \sum_{m=1}^{n_{p}} \mathbb{1}_{\left\{X_{m}^{p} \geq 2\right\}}-\hat{h}^{n_{p}}(1) \sum_{m=1}^{n_{p}} \mathbb{1}_{\left\{X_{m}^{p} \geq 1\right\}}\right]=0 \\
& R^{n_{p}}(2)-\left[\hat{h}^{n_{p}}(2) V^{n_{p}}(2)-\hat{h}^{n_{p}}(1) V^{n_{p}}(1)\right]=0 \\
& R^{n_{p}}(2)-\left[\hat{h}^{n_{p}}(2) V^{n_{p}}(2)-\frac{R^{n_{p}}(1)}{V^{n_{p}}(1)} V^{n_{p}}(1)\right]=0 \\
& \Rightarrow \quad \hat{h}^{n_{p}}(2)=\frac{\Delta R^{n_{p}}(2)}{V^{n_{p}}(2)} \mathbb{1}_{\left\{V^{n_{p}}(2)>0\right\}}
\end{aligned}
$$

em que $\Delta R^{n_{p}}(2)=R^{n_{p}}(2)-R^{n_{p}}(1)$. Calculando analogamente, obtemos o Kaplan-Meier

$$
\hat{h}^{n_{p}}(i)=\frac{\Delta R^{n_{p}}(i) J^{n_{p}}(i)}{V^{n_{p}}(i)} ; \quad i \geq 1,
$$

no qual $\Delta R^{n_{p}}(i)=R^{n_{p}}(i)-R^{n_{p}}(i-1)$ e $J^{n_{p}}(i)=\mathbb{1}_{\left\{V^{n_{p}}(i)>0\right\}}$ para $i \geq 1$. A seguir, apresentamos dois resultados, que são importantes na demonstração da consistência dos testes sob certas hipóteses alternativas.

\section{Hipótese 2.1. (Proporcionalidade do tamanho da amostra)}

Todos os resultados assintóticos descritos no capítulo 3, tem como hipótese o fato de que existe o limite

$b_{p}=\lim _{n^{\star} \rightarrow \infty} \frac{n_{p}}{n}$ e $X^{p}$ é integrável para todo $p \in \mathcal{J}$. Isto que dizer que as amostras aumentam de tamanho de forma proporcional.

Proposição 2.1.1. O número de ítens sob risco na categoria $\ell$, denotado por $V^{n_{p}}(\ell)$ tem distribuição binomial com parâmetros $n_{p}$ e $\theta_{\ell}^{p}$, em que $\theta_{\ell}^{p}=P\left[X^{p} \geq \ell\right]$ para cada $\ell \geq 1$.

Demonstração. Seja a variável aleatória $V_{m}^{p}$, que assume apenas os valores 0 e 1 . Assim, afirmamos que $V_{m}^{p}(i) \sim$ Bernoulli com parâmetro $\theta_{i}^{p}$.

Logo, por (2.10), temos que é soma de variáveis aleatórias com distribuição de Bernoulli independentes. Portanto, temos que $V^{n_{p}}(\ell) \sim \operatorname{Binomial}\left(n_{p}, \theta_{\ell}^{p}\right)$

Agora, apresentamos dois resultados, fixamos $p$ e para $\ell=1, \ldots, k$ temos que

$$
\mathbf{E}\left[\frac{V^{n_{p}}(\ell)}{n_{p}}\right] \stackrel{\text { Proposição }}{=}(2.1 .1) \theta_{\ell}^{p}
$$

Já para o segundo resultado, temos que para $\ell=1, \ldots, k$ e $p=1, \ldots, J$ obtemos

$$
\begin{aligned}
\frac{V^{n^{\star}}(\ell)}{n_{p}} & =\frac{\left[V^{n_{1}}(\ell)+V^{n_{2}}(\ell)+\cdots+V^{n_{J}}(\ell)\right]}{n_{p}}= \\
& =\frac{n_{1}}{n_{p}} \frac{V^{n_{1}}(\ell)}{n_{1}}+\frac{n_{2}}{n_{p}} \frac{V^{n_{2}}(\ell)}{n_{2}}+\cdots+\frac{n_{J}}{n_{p}} \frac{V^{n_{J}}(\ell)}{n_{J}}
\end{aligned}
$$


Logo, usando (2.12) e (2.13) temos o seguinte resultado

$$
\begin{array}{r}
\mathbf{E}\left[\frac{V^{n^{\star}}(\ell)}{n_{p}}\right]=\frac{n_{1}}{n_{p}} \mathbf{E}\left[\frac{V^{n_{1}}(\ell)}{n_{1}}\right]+\frac{n_{2}}{n_{p}} \mathbf{E}\left[\frac{V^{n_{2}}(\ell)}{n_{2}}\right]+\cdots+\frac{n_{J}}{n_{p}} \mathbf{E}\left[\frac{V^{n_{J}}(\ell)}{n_{J}}\right] \\
\stackrel{\text { Hipótese } 2.1}{\longrightarrow} b_{1} \theta_{\ell}^{1}+b_{2} \theta_{\ell}^{2}+\cdots+b_{J} \theta_{\ell}^{J}=\gamma^{n_{p}}(\ell)
\end{array}
$$

Nosso interesse consiste em testar a hipótese de homogeneidade das $J$ populações discretas sujeitas a censura aleatória. Neste caso, queremos testar a hipótese $H_{0}: \pi_{\ell}^{1}=\cdots=\pi_{\ell}^{J} \quad \forall \ell=1, \ldots, k-1$. Como a função intensidade caracteriza a variável aleatória discreta, a hipótese de homogeneidade é equivalente a $H_{0}: h^{1}(\ell)=\cdots=h^{J}(\ell) \quad \forall \ell=1, \ldots, k-1$, no qual $k$ pode ser finito ou não. No próximo capítulo, vamos descrever os testes não paramétricos usados neste trabalho. 


\section{Capítulo 3}

\section{Comparação de dados discretos na}

\section{presença de censura}

Este capítulo é dedicado principalmente em explicar a construção das estatísticas de Cramér-von Mises e Log-rank Ponderado, baseado no artigo de Leão e Ohashi [16], que são aplicados a quaisquer espaços amostrais discretos. Outro ponto importante neste capítulo é a construção de uma modificação no teste de Log-rank Ponderado utilizado para espaços amostrais finitos, que são aplicados principalmente em dados discretos dispostos em uma tabela de contingência. As estatísticas deste capítulo têm como base a teoria de Martingale e Processos de Contagem, e os principais detalhes matemáticos podemos encontrar no artigo de Leão e Ohashi [16]. Na primeira seção introduzimos a estatística de Log-rank Ponderado, em seguida propomos uma modificação na estatística de Log-rank, enunciamos a estatística de Cramér-von Mises e por fim, apresentamos um algoritmo para calcularmos os testes enunciados neste capítulo.

\subsection{A estatística de Log-rank Ponderado}

Nesta seção, vamos estender as estatísticas de Log-rank Ponderadas proposta por Fleming e Harrington (1990) [5] para comparar $J$ populações discretas na presença de censura arbitrária. Lembramos que $R^{n^{\star}}(i)=R^{n_{1}}(i)+\cdots+R^{n_{J}}(i)$ é o número total de eventos de interesse da categoria $i$ e que $V^{n^{\star}}(i)=V^{n_{1}}(i)+\cdots+V^{n_{J}}(i)$ é o número total de ítens sob Risco na categoria $i$, para qualquer $i=1,2, \ldots, k$. Além disso, denotamos por $n^{\star}=\left(n_{1}, n_{2}, \cdots, n_{J}\right)$ o vetor de números naturais correspondente ao tamanho da amostra retirado de cada população e $n=n_{1}+n_{2}+\cdots n_{J}$ o total de elementos nas amostras. Também dizemos que $n^{\star} \rightarrow \infty$ se $n_{p} \rightarrow \infty$ para todo $p=1 \cdots, J$. Vamos derivar uma classe de estatísticas de teste para a hipótese $H_{0}: h^{1}(\ell)=h^{2}(\ell)=\cdots=h^{J}(\ell)$, para todo $\ell=1, \ldots, i$, no qual $i$ pode ser finito ou não. A seguir, denotamos a sequência de $\mathbb{F}$ tempos de parada 


$$
\begin{aligned}
d^{u} & =\sup \left\{\ell: \min _{q \in \mathcal{J}} \theta^{q}(\ell)>0\right\}, \\
\text { em que } \theta^{q}(\ell) & :=P\left[X^{q} \geq \ell\right], \quad q \in\{1, \ldots, J\}, \ell \geq 0 \mathrm{e} \\
d_{n^{\star}}^{u} & =\sup \left\{\ell: \min _{q \in \mathcal{J}} V^{n_{q}}(\ell)>0\right\}, n^{\star} \in \mathbb{N}^{J} .
\end{aligned}
$$

Podemos verificar que $d_{n^{\star}}^{u} \rightarrow d^{u}$ em probabilidade quando $n^{\star} \rightarrow \infty$, em que $1 \leq d^{u} \leq \infty$ e $d_{n^{\star}}^{u}<$ $\infty$ para todo $n^{\star} \in \mathbb{N}^{J}$ (propriedades $S_{1}$ e $S_{2}$ do artigo de Leão e Ohashi [16]). É importante ressaltar que todos os resultados assintóticos deste trabalho são obtidos com a hipótese de proporcionalidade (2.1). Considerando o problema de duas populações $(J=2)$, Fleming e Harrington (1990)[5] propuseram as seguintes estatísticas de Log-rank Ponderada,

$$
L R\left(n^{\star}, d_{n^{\star}}^{u}\right)=\sum_{\ell=1}^{i} U_{n_{2}}^{n_{1}}\left(n^{\star}, \ell\right)\left[\hat{h}^{n_{2}}(\ell)-\hat{h}^{n_{1}}(\ell)\right]
$$

com função de ponderação dada por

$$
U_{n_{2}}^{n_{1}}\left(n^{\star}, \ell\right)=\left(\frac{1}{n}\right)^{1 / 2} u\left(n^{\star}, \ell\right)\left(\frac{V^{n_{1}}(\ell) V^{n_{2}}(\ell)}{V^{n^{\star}}(\ell)}\right) \mathbb{1}_{\left\{V^{n_{1}}(\ell)>0\right\}}
$$

em que $u$ é um processo previsível, limitado, que converge em probabilidade para uma função limitada $w:\{0,1,2, \cdots, k\} \rightarrow \mathbb{R}$ e $n=n_{1}+n_{2}$. Uma das principais ponderações é a classe de Tarone-Ware (1977) [?],

$$
u\left(n^{\star}, \ell\right)=\left(\frac{V^{n^{\star}}(\ell)}{n}\right)^{\gamma}, \quad \text { em que } n=\sum_{i=1}^{J} n_{i}, \gamma>0,
$$

e as classes introduzidas por Harrington e Fleming (1982) [10],

$$
u\left(n^{\star}, \ell\right)=\left[\prod_{j=0}^{\ell-1}\left(1-\frac{\Delta R^{n^{\star}}(j)}{V^{n^{\star}}(j)}\right)\right]^{\rho}\left[1-\prod_{j=0}^{\ell-1}\left(1-\frac{\Delta R^{n^{\star}}(j)}{V^{n^{\star}}(j)}\right)\right]^{\gamma}, \quad \gamma \geq 0, \rho \geq 0 .
$$

Se tomarmos $u\left(n^{\star}, \ell\right)=1$ para todo $\ell$, obtemos a clássica estatística de Log-rank. Na tabela (3.1) temos as funções de ponderação mais utilizadas (ver Klein e Moeschberger [21] página 210).

A escolha do processo de ponderação $u\left(n^{\star}, \ell\right)$ determina a função poder da estatística do teste contra diferentes hipóteses alternativas. A estatística escolhida será mais sensível com relação às diferenças entre as funções intensidades nas categorias em que $u\left(n^{\star}, \cdot\right)$ é grande e menos sensível nas categorias em que $u\left(n^{\star}, \cdot\right)$ é pequena. Para os processos de ponderação do tipo Fleming e Harrington com $\rho>0, \gamma=0$, intuitivamente, têm um bom poder para detectar precocemente diferenças (entre as funções intensidades) que desaparecem ao longo do tempo. Por outro lado, com o mesmo processo mas $\rho=0, \gamma>0$ será mais poderosa contra as alternativas com funções intensidade não proporcionais. O processo de ponderação do tipo log-rank, tem desempenho satisfatório para alternativas com funções de intensidade constantes ou 


\begin{tabular}{ccc}
\hline Peso & $u\left(n^{\star}, \ell\right)$ & Parâmetros \\
\hline Log-rank & 1 & \\
Gehan & $\frac{V^{n^{\star}}(\ell)}{n}$ & \\
Tarone e Ware & $\left(\frac{V^{n^{\star}}(\ell)}{n}\right)^{\gamma}$ & \\
Peto e Peto & $\prod_{j=1}^{\ell-1}\left(1-\left(\frac{\Delta R^{n^{\star}}(j)}{1+V^{n^{\star}}(j)}\right)\right)$ & \\
Peto e Peto Modificado & $\prod_{j=1}^{\ell-1}\left(1-\left(\frac{\Delta R^{n^{\star}}(j)}{1+V^{n^{\star}}(j)}\right)\right)\left[\frac{\left(V^{n^{\star}}(j)\right)}{1+\left(V^{n^{\star}}(j)\right)}\right]$ & \\
Fleming e Harrington & {$\left[\prod_{j=0}^{\ell-1}\left(1-\frac{\Delta R^{n^{\star}}(j)}{V^{n^{\star}}(j)}\right)\right]^{\rho}\left[1-\prod_{j=0}^{\ell-1}\left(1-\frac{\Delta R^{n^{\star}}(j)}{V^{n^{\star}}(j)}\right)\right]^{\gamma}$} & \\
& $\gamma \geq 0, \rho \geq 0$
\end{tabular}

Tabela 3.1: Funções de ponderação.

proporcionais.

Agora, vamos tratar para o problema de $J$ populações. Para testarmos a hipótese nula $H_{0}: h^{1}=\cdots=$ $h^{J}$, derivamos uma generalização da estatística de Log-rank ponderada proposta por Fleming e Harrington (2005)[5] que pode ser encontrada em Andersen et al. [3]. Usando a função de ponderação

$$
U_{n_{q_{1}}}^{n_{q}}\left(n^{\star}, \ell\right)=\left(\frac{1}{n}\right)^{\frac{1}{2}} u\left(n^{\star}, \ell\right)\left(\frac{V^{n_{q}}(\ell) V^{n_{q_{1}}}(\ell)}{V^{n^{\star}}(\ell)}\right) \mathbb{1}_{\left\{V^{\left.n_{q}(\ell)>0\right\}}\right.}, \quad \text { em que } n=n_{1}+\cdots+n(3.4)
$$

obtemos

$$
\begin{aligned}
L R_{q}\left(n^{\star}, j\right) & :=\sum_{q_{1} \neq q} \sum_{\ell=1}^{j} U_{n_{q_{1}}}^{n_{q}}\left(n^{\star}, \ell\right)\left[\hat{h}^{n_{q}}(\ell)-\hat{h}^{n_{q_{1}}}(\ell)\right] \\
& =\sum_{\ell=1}^{j}\left(\frac{1}{n}\right)^{1 / 2} u\left(n^{\star}, \ell\right)\left[\Delta R^{n_{q}}(\ell)-V^{n_{q}}(\ell) \frac{\Delta R^{n^{\star}}(\ell)}{V^{n^{\star}}(\ell)}\right] \\
& =\sum_{\ell=1}^{j}\left(\frac{1}{n}\right)^{1 / 2} u\left(n^{\star}, \ell\right) V^{n_{q}}(\ell)\left[\frac{\Delta R^{n_{q}}(\ell)}{V^{n_{q}}(\ell)}-\frac{\Delta R^{n^{\star}}(\ell)}{V^{n^{\star}}(\ell)}\right], n^{\star} \in \mathbb{N}^{J}, j \geq 1 .
\end{aligned}
$$

Agora, segue do Teorema (3.1) e Proposição (2.1.1) (Leão e Ohashi (2012) [16]), que sob $H_{0}$ o vetor aleatório

$$
L R\left(n^{\star}, d_{n^{\star}}^{u}\right):=\left(L R_{1}\left(n^{\star}, d_{n^{\star}}^{u}\right), \ldots, L R_{J}\left(n^{\star}, d_{n^{\star}}^{u}\right)\right)^{T}
$$

converge em distribuição para $N\left(0, \Gamma\left(d^{u}\right)\right)$ quando $n^{\star} \rightarrow \infty$, em que $\Gamma\left(d^{u}\right)$ admite um estimador consistente $\hat{\Gamma}\left(n^{\star}, d_{n^{\star}}^{u}\right)$, na forma $\hat{\Gamma}\left(n^{\star}, i\right):=\sum_{\ell=1}^{i} \hat{Q}(\ell), i \geq 1 \mathrm{com}$ 


$$
\hat{Q}(\ell)_{i, j}=\left\langle\hat{Q}(\ell) a_{i}, a_{j}\right\rangle
$$

para todo $a \in \mathbb{R}^{J} \mathrm{e}\langle\cdot, \cdot\rangle_{\mathbb{R}^{J}}$ o produto interno no $\mathbb{R}^{J}$. A forma matricial é dada por

$$
\left[\hat{Q}\left(n^{\star}, \ell\right)\right]_{r k}:=\left\{\begin{array}{cc}
\hat{\phi}_{k, n^{\star}}^{2}(\ell) ; & \text { se } r=k ; \\
\hat{\psi}_{n^{\star}}^{2}(k, r, \ell) ; & \text { se } r \neq k ;
\end{array}\right.
$$

em que

$$
\begin{aligned}
\hat{\phi}_{q, n^{\star}}^{2}(\ell) & :=\sum_{q_{1} \neq q}\left[\frac{\left|U_{n_{q_{1}}}^{n_{q}}\left(n^{\star}, \ell\right)\right|^{2}}{V_{q_{1}}(\ell)} \hat{h}^{n_{q_{1}}}(\ell)\left[1-\hat{h}^{n_{q_{1}}}(\ell)\right]+\frac{\left|U_{n_{q_{1}}}^{n_{q}}\left(n^{\star}, \ell\right)\right|^{2}}{V^{n_{q}}(\ell)} \hat{h}^{n_{q}}(\ell)\left[1-\hat{h}^{n_{q}}(\ell)\right]\right]+ \\
& +2 \sum_{q_{1}, q_{2} \in A_{q}} \frac{U_{n_{q_{1}}}^{n_{q}}\left(n^{\star}, \ell\right) U_{n_{2}}^{n_{q}}\left(n^{\star}, \ell\right)}{V^{n_{q}}(\ell)} \hat{h}^{n_{q}}(\ell)\left[1-\hat{h}^{n_{q}}(\ell)\right], \quad \ell \geq 1
\end{aligned}
$$

no qual, $A_{q}=\{(x, y) \in \mathcal{J} \times \mathcal{J} ; x \neq y, x \neq q, y \neq q, 1 \leq x<y \leq J\}$ e $\mathcal{J}=\{1, \ldots, J\}$.

Além disso,

$$
\begin{aligned}
\hat{\psi}_{n^{\star}}(k, r, \ell) & :=\sum_{q_{1}, q_{2} \in A(k, r)} \frac{U_{n_{q_{1}}}^{n_{k}}\left(n^{\star}, \ell\right) U_{n_{q_{1}}}^{n_{r}}\left(n^{\star}, \ell\right)}{V^{n_{q_{1}}(\ell)}} \mathbb{1}_{\left\{V^{\left.n_{q_{1}}(\ell)>0\right\}}\right.} \hat{h}^{q_{1}}(\ell)\left[1-\hat{h}^{q_{1}}(\ell)\right]- \\
& -\sum_{q_{1} \neq k} \frac{U_{n_{q_{1}}}^{n_{k}}\left(n^{\star}, \ell\right) U_{n_{k}}^{n_{r}}\left(n^{\star}, \ell\right)}{V^{n_{k}}(\ell)} \mathbb{1}_{\left\{V^{n_{k}}(\ell)>0\right\}} \hat{h}^{k}(\ell)\left[1-\hat{h}^{k}(\ell)\right]- \\
& -\sum_{q_{2} \neq r} \frac{U_{n_{q_{2}}}^{n_{r}}\left(n^{\star}, \ell\right) U_{n_{r}}^{n_{k}}\left(n^{\star}, \ell\right)}{V^{n_{r}}(\ell)} \mathbb{1}_{\left\{V^{\left.n_{r}(\ell)>0\right\}}\right.} \hat{h}^{r}(\ell)\left[1-\hat{h}^{r}(\ell)\right], \quad \ell \geq 1
\end{aligned}
$$

em que,

$$
A(k, r)=\left\{\left(q_{1}, q_{2}\right) \in \mathcal{J} \times \mathcal{J} ; q_{1} \neq k, q_{2} \neq r, q_{1}=q_{2}\right\} \text { e } \mathcal{J}=\{1, \ldots, J\} .
$$

No entanto, como uma consequência da definição da estatística $L R_{q}\left(n^{\star}, d_{n^{\star}}^{u}\right)$, temos que $\sum_{q=1}^{J} L R_{q}\left(n^{\star}, d_{n^{\star}}^{u}\right)=$ 0. Assim, o vetor aleatório das estatísticas de Log-rank Ponderado é linearmente dependente e a matriz de covariância assintótica $\Gamma\left(n^{\star}, d_{n^{\star}}^{u}\right)$ tem posto não superior a $J-1$. Sob condições gerais sobre $h^{p}$ $(p=1, \ldots, J)$, tal como a existência para qualquer $q, p=1, \ldots, J$ de pelo menos um índice $\ell \leq i$ tal que $h^{p}(\ell)>0$ e $h^{q}(\ell)>0$, pode ser provado que o posto de $\Gamma(i)$ é $J-1$, para qualquer $i \geq 1$ (ver, Gill (1986)[7], Apêndice 1). Da mesma forma, o estimador da covariância

$$
\hat{\Gamma}\left(n^{\star}, i\right):=\sum_{\ell=1}^{i} \hat{Q}\left(n^{\star}, \ell\right), \quad i \geq 1
$$

tem posto $J-1$. Além disso, para qualquer $q, p=1, \ldots, J$, existe pelo menos um índice $\ell \leq i$ tal que $\Delta R^{n_{p}}(\ell)$ e $\Delta R^{n_{q}}(\ell)$ são positivos. Além disso, como $\hat{\Gamma}\left(n^{\star}, i\right)$ é um estimador consistente de $\Gamma(i)$, obtemos 
que a probabilidade de $\hat{\Gamma}\left(n^{\star}, i\right)$ converge para 1 quando $n^{\star}$ tende ao infinito. Assim, sob $H_{0}$, a estatística

$$
X^{2}\left(n^{\star}, d_{n^{\star}}^{u}\right)=L R\left(n^{\star}, d_{n^{\star}}^{u}\right)^{T} \hat{\Gamma}\left(n^{\star}, d_{n^{\star}}^{u}\right)^{-1} L R\left(n^{\star}, d_{n^{\star}}^{u}\right)
$$

tem distribuição assintótica Qui-Quadrado com $J-1$ graus de liberdade, no qual $\hat{\Gamma}^{-1}\left(n^{\star}, d_{n^{\star}}^{u}\right)$ é uma inversa generalizada. Portanto, se excluirmos a última linha e última coluna de $\hat{\Gamma}\left(n^{\star}, d_{n^{\star}}^{u}\right)$, resultando em $\hat{\Gamma}_{0}\left(n^{\star}, d_{n^{\star}}^{u}\right)=\sum_{\ell=1}^{j} \hat{Q}_{0}\left(n^{\star}, \ell\right), j \geq 1$, obtemos por consistência de $\hat{\Gamma}\left(n^{\star}, d_{n^{\star}}^{u}\right)$ que o posto é $J-1$, ou seja, a probabilidade de $\hat{\Gamma}$ ter posto $J-1$ cresce para 1 quando $n^{\star} \rightarrow \infty$. Assim, tomando o vetor aleatório

$$
L R_{0}\left(n^{\star}, d_{n^{\star}}^{u}\right)=\left(L R_{1}\left(n^{\star}, d_{n^{\star}}^{u}\right), \ldots, L R_{J-1}\left(n^{\star}, d_{n^{\star}}^{u}\right)\right)^{T}
$$

concluímos que

$$
X^{2}\left(n^{\star}, d_{n^{\star}}^{u}\right)=L R_{0}\left(n^{\star}, d_{n^{\star}}^{u}\right)^{T} \hat{\Gamma}_{0}\left(n^{\star}, d_{n^{\star}}^{u}\right)^{-1} L R_{0}\left(n^{\star}, d_{n^{\star}}^{u}\right)
$$

tem distribuição assintótica Qui-Quadrado com $J-1$ graus de liberdade, no qual $\hat{\Gamma}_{0}\left(n^{\star}, d_{n^{\star}}^{u}\right)^{-1}$ é a inversa clássica.

Para investigarmos o poder do teste, removemos o pressuposto de $H_{0}$. Neste caso, temos que

$$
\begin{aligned}
& L R_{q}\left(n^{\star}, i\right)=\sum_{\ell=1}^{i} \sum_{q_{1} \neq q} U_{n_{q_{1}}}^{n_{q}}\left(n^{\star}, \ell\right)\left[\hat{h}^{n_{q}}(\ell)-\hat{h}^{n_{q_{1}}}(\ell)\right]= \\
& =\sum_{\ell=1}^{i} \sum_{q_{1} \neq q} U_{n_{q_{1}}}^{n_{q}}\left(n^{\star}, \ell\right)\left[\frac{\Delta Y^{n_{q}}(\ell) \mathbb{1}_{\left\{V^{n_{q}}(\ell)>0\right\}}}{V^{n_{q}}(\ell)}-\frac{\Delta Y^{n_{q_{1}}(\ell) \mathbb{1}_{\left\{V^{n_{q_{1}}}(\ell)>0\right\}}}}{V^{n_{q_{1}}(\ell)}}\right]+ \\
& +\sum_{\ell=1}^{i} \sum_{q_{1} \neq q} U_{n_{q_{1}}}^{n_{q}}\left(n^{\star}, \ell\right)\left[h^{q}(\ell)-h^{q_{1}}(\ell)\right], i \in \mathcal{K} .
\end{aligned}
$$

Seja $\left\{Q_{n}: n \in \mathbb{N}\right\}$ uma sequência de testes estatísticos e $\left\{R_{n}: n \in \mathbb{N}\right\}$ as respectivas regiões de rejeição associadas a um nível de significância fixado. Dizemos que uma sequência de estatísticas de teste $Q_{n}$ é consistente com uma hipótese alternativa $H_{1}$ se $\lim _{n} P\left(Q_{n} \in R_{n} \mid H_{1}\right)=1$, veja Fleming e Harrington (1991) [5] para mais detalhes. A classe de hipóteses alternativas $H_{1}: h^{1}(\ell)>h^{q}(\ell)$, para qualquer $\ell=1, \cdots, k-1$ e $q=2, \cdots, J$ é chamado de hipóteses alternativas de intensidades ordenadas.

Lema 3.1.1. Sob hipóteses alternativas de intensidades ordenadas, o teste de log-rank ponderado $X^{2}(k-$ 1) é consistente, para $k$ finito ou não.

Demonstração. Para tanto, é suficiente para provar que

$$
\sum_{\ell=1}^{k-1} \sum_{q_{1} \neq 1} U_{n_{q_{1}}}^{n_{1}}\left(n^{\star}, \ell\right)\left[h^{1}(\ell)-h^{q_{1}}(\ell)\right] \rightarrow \infty, \quad \text { em probabilidade }
$$

quando $n^{\star}$ tende ao infinito. 
Como temos por hipótese, que as alternativas de intensidade são ordenadas, então temos que

$$
\left[h^{1}(\ell)-h^{q_{1}}(\ell)\right]>0
$$

Agora, basta provar que $U_{n_{1}}^{n_{1}}\left(n^{\star}, \ell\right) \rightarrow \infty$ quando $n^{\star}$ tende ao infinito.

De fato, primeiramente vamos usar os seguinte resultados:

1. $u\left(n^{\star}, \ell\right) \longrightarrow w$ em probabilidade, em que $w: \mathcal{K} \longmapsto \mathbb{R}$ é uma função limitada;

2. Resultado (2.14) e proposição (2.1.1).

Portanto, temos que

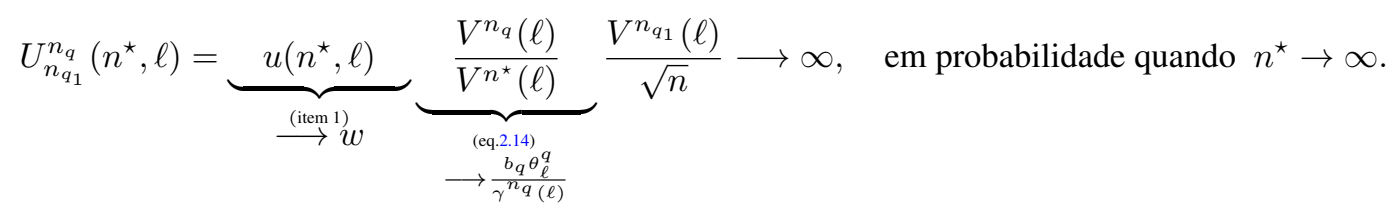

em que $n=n_{1}+\cdots+n_{J}$

\subsection{A estatística de Log-rank Modificado}

O teste de Log-rank Ponderado pode não ter um bom comportamento quando há cruzamento nas funções de intensidade. Na seção (3.2), realizamos um estudo de simulação para exemplificar este fato. Para resolver este problema, propomos uma modificação nas estatísticas de Log-rank Ponderado para tratarmos espaços amostrais finitos $(\ell=1, \cdots, k$ (Finito)). Primeiramente definimos

$$
M L R^{q}\left(n^{\star}, \ell\right)=\sum_{q_{1} \neq q} U_{n_{q_{1}}}^{n_{q}}\left(n^{\star}, \ell\right)\left[\hat{h}^{n_{q}}(\ell)-\hat{h}^{n_{q_{1}}}(\ell)\right], \ell=1, \cdots, k .
$$

Decorre do Teorema (3.1) (Leão e Ohashi [16]) que, sob $H_{0}, M L R^{q}\left(n^{\star}, \ell\right)$ tem distribuição assintótica normal com média zero e matriz de covariância $Q(\ell)$, cujo estimador $\hat{Q}(\ell)$ está definido na Equação (3.6). Além disso, o martingale $M L R^{q}\left(n^{\star}, \cdot\right)$ tem incrementos assintoticamente independentes. Nesse caso, se denotarmos

$$
M L R_{0}\left(n^{\star}, \ell\right)=\left(M L R^{1}\left(n^{\star}, \ell\right), M L R^{2}\left(n^{\star}, \ell\right), \ldots, M L R^{J-1}\left(n^{\star}, \ell\right)\right)^{T},
$$

obtemos a estatística de Log-rank Modificado

$$
M X^{2}\left(n^{\star}, k-1\right)=\sum_{\ell=1}^{k-1} M L R_{0}\left(n^{\star}, \ell\right)^{T} \hat{Q}_{0}\left(n^{\star}, \ell\right)^{-1} M L R_{0}\left(n^{\star}, \ell\right), \quad \ell \in\{1,2, \ldots, k-1\},
$$

que tem distribuição assintótica Qui-Quadrado com $(J-1) \times(k-1)$ graus de liberdade, no qual $\hat{Q}_{0}\left(n^{\star}, \ell\right)$ 
é o operador de covariância $\hat{Q}\left(n^{\star}, \ell\right)$ sem a última linha e a última coluna, assim como argumentado na seção anterior, e denotamos $\hat{Q}_{0}\left(n^{\star}, \ell\right)^{-1}$ como a inversa.

Com esse tipo de modificação em relação ao Log-rank Ponderado proposto por Fleming e Harrington (1990)[5] para duas populações (J=2) e estendido por Leão e Ohashi [16] para J populações discretas, resolvemos o problema das diferenças iniciais canceladas em favor da outra população, como dita no capítulo (1). Portanto, resolvemos o problema do cruzamento das funções de intensidade para espaços amostrais finitos. Para o caso de espaços amostrais infinitos enumeráveis, usamos a estatística de Cramér-von Mises proposta por Leão e Ohashi [16].

Ao retirarmos a hipótese $H_{0}$, chegamos a seguinte equação

$$
\begin{aligned}
& M L R^{q}\left(n^{\star}, \ell\right)=\sum_{q_{1} \neq q} U_{n_{q_{1}}}^{n_{q}}\left(n^{\star}, \ell\right)\left[\hat{h}^{n_{q}}(\ell)-\hat{h}^{n_{q_{1}}}(\ell)\right]= \\
& =\sum_{q_{1} \neq q} U_{n_{q_{1}}}^{n_{q}}\left(n^{\star}, \ell\right)\left[\frac{\Delta Y^{n_{q}}(\ell) \mathbb{1}_{\left\{V^{n_{q}}(\ell)>0\right\}}}{V^{n_{q}}(\ell)}-\frac{\Delta Y^{n_{q_{1}}}(\ell) \mathbb{1}_{\left\{V^{n_{q_{1}}}(\ell)>0\right\}}}{V^{n_{q_{1}}(\ell)}}\right]+ \\
& +\sum_{q_{1} \neq q} U_{n_{q_{1}}}^{n_{q}}\left(n^{\star}, \ell\right)\left[h^{q}(\ell)-h^{q_{1}}(\ell)\right], \ell \in \mathcal{K} \text {. }
\end{aligned}
$$

Assim como na seção (3.1), definimos $\left\{Q_{n}: n \in \mathbb{N}\right\}$ uma sequência de testes estatísticos e $\left\{R_{n}\right.$ : $n \in \mathbb{N}\}$ as respectivas regiões de rejeição associadas a um nível de significância fixado. Dizemos que uma sequência de estatísticas de teste $Q_{n}$ é consistente com uma hipótese alternativa $H_{1}$ se $\lim _{n} P\left(Q_{n} \in R_{n} \mid\right.$ $\left.H_{1}\right)=1$. Assim, a classe de hipóteses alternativas é definida como

\section{Definição 3.1. (Hipótese alternativa de intensidades)}

Existe pelo menos um par de índices $q, q_{1}=1, \cdots, J$ com $q \neq q_{1}$ para os quais $H_{1}: h^{q}(\ell) \neq h^{q_{1}}(\ell)$, para algum $\ell=1, \cdots, k-1$.

Assim, mostramos a consistência através do seguinte teorema.

Teorema 3.2.1. No caso particular de duas populações $(J=2)$, temos que o teste de Log-rank modificado $M X^{2}\left(n^{\star}, k-1\right)$ é consistente para qualquer hipótese alternativa (3.1).

Demonstração. De fato, tomamos o caso de duas populações $(\mathrm{J}=2)$.

A seguir, vamos avaliar $\mathbf{E}\left[\left(M L R^{1}\left(n^{\star}, i\right)\right)^{2}\right]$, e lembrando que $\sum_{k=1}^{J} \hat{\phi}_{k, n^{\star}}^{2}(\ell)<\infty$. Então

$$
\mathbf{E}\left[\left(M L R^{1}\left(n^{\star}, i\right)\right)^{2}\right] \geq\left[\mathbf{E}\left(M L R^{1}\left(n^{\star}, i\right)\right)\right]^{2}
$$

Calculando o valor esperado, temos que

$$
\begin{aligned}
\mathbf{E}\left[M L R^{1}\left(n^{\star}, i\right)\right]= & \mathbf{E}\left(U_{n_{2}}^{n_{1}}\left(n^{\star}, \ell\right)\left[\frac{\Delta Y^{n_{1}}(\ell) \mathbb{1}_{\left\{V^{n_{1}}(\ell)>0\right\}}}{V^{n_{1}}(\ell)}-\frac{\Delta Y^{n_{2}}(\ell) \mathbb{1}_{\left\{V^{n_{2}}(\ell)>0\right\}}}{V^{n_{2}}(\ell)}\right]\right)+ \\
& +\mathbf{E}\left(U_{n_{2}}^{n_{1}}\left(n^{\star}, \ell\right)\left[h^{1}(\ell)-h^{2}(\ell)\right]\right)
\end{aligned}
$$


Usando o fato de $Y^{n_{1}}$ e $Y^{n_{2}}$ serem martingales e o processo de ponderação $U_{n_{2}}^{n_{1}}$ ser previsível, obtemos

$$
\left[\mathbf{E}\left(M L R^{1}\left(n^{\star}, i\right)\right)\right]^{2}=\mathbf{E}\left[U_{n_{2}}^{n_{1}}\left(n^{\star}, \ell\right)\right]^{2}\left[h^{1}(\ell)-h^{2}(\ell)\right]^{2}
$$

No caso particular de duas populações, sob condições das hipóteses alternativas (3.1), temos que Logrank Modificado é consistente, pois temos $\left[h^{1}(\ell)-h^{2}(\ell)\right]^{2}>0$ para algum $\ell=1, \cdots, k-1 \mathrm{e}\left[U_{n_{q_{1}}}^{n_{q}}\left(n^{\star}, \ell\right)\right]^{2}$ $\rightarrow \infty$. Este último resultado pode ser mostrado usando as mesmas propriedades do lema (3.1.1), ou seja,

$$
\left[U_{n_{q_{1}}}^{n_{q}}\left(n^{\star}, \ell\right)\right]^{2}=u^{2}\left(n^{\star}, \ell\right)\left(\frac{V^{n_{q}}(\ell)}{V^{n^{\star}}(\ell)}\right)^{2} \frac{V^{n_{q_{1}}}(\ell)}{\sqrt{n}} \frac{V^{n_{q_{1}}}(\ell)}{\sqrt{n}} \longrightarrow \infty
$$

em probabilidade quando $n^{\star} \rightarrow \infty$,

Portanto, para $J=2$, temos que o teste de Log-rank modificado $M X^{2}\left(n^{\star}, k-1\right)$ é consistente para qualquer hipótese alternativa.

Testes baseados em estatísticas que sejam consistentes contra todas as alternativas têm o poder de convergindo para 1 conforme o tamanho das amostras convergem para o infinito. A seguir, ilustramos a estatística de Log-rank modificada através de aplicações para espaços amostrais finitos.

\section{Exemplo 3.1. (Teste de Homogeneidade em tabelas $2 \times 2$ )}

Suponhamos que $n_{1}+n_{2}$ observações de dados estão resumidos em uma tabela $2 \times 2$,

\begin{tabular}{lccc}
\hline & Categoria 1 & Categoria 2 & Total \\
& & \\
\hline Pop 1 & $\Delta R^{n_{1}}(1)$ & $\Delta R^{n_{1}}(2)$ & $n_{1}=V^{n_{1}}(1)$ \\
Pop 2 & $\Delta R^{n_{2}}(1)$ & $\Delta R^{n_{2}}(2)$ & $n_{2}=V^{n_{2}}(2)$ \\
\hline Total & $\Delta R^{n^{\star}}(1)$ & $\Delta R^{n^{\star}}(2)$ & $n_{1}+n_{2}=V^{n^{\star}}$ \\
\hline
\end{tabular}

Tabela 3.2: Tabela de contingência $2 \times 2$.

Vamos admitir que cada amostra é uma amostra aleatória, sendo elas independentes entre si e cada observação pode ser classificada na categoria 1 ou na categoria 2 , mas não em ambas. Seja $\pi_{1}^{1}$ a probabilidade de que um elemento da população 1 seja classificado na categoria 1 e $\pi_{1}^{2}$ o correspondente da população 2. Neste exemplo, queremos testar a hipótese $H_{0}: \pi_{1}^{1}=\pi_{1}^{2}$, ou equivalentemente, $H_{0}: h^{1}(1)=h^{2}(1)$. Então, temos a seguinte estatística de Log-rank $\left(u\left(n^{\star}, 1\right)=1\right)$

$$
\begin{aligned}
\operatorname{MLR}\left(n^{\star}, 1\right) & =\left(\frac{1}{n_{1}+n_{2}}\right)^{1 / 2}\left(\frac{V^{n_{1}}(1) V^{n_{2}}(1)}{V^{n^{\star}}}\right)\left[\hat{h}^{n_{2}}(1)-\hat{h}^{n_{1}}(1)\right]= \\
& =\left(\frac{1}{n_{1}+n_{2}}\right)^{1 / 2}\left(\frac{V^{n_{1}}(1) V^{n_{2}}(1)}{V^{n^{\star}}}\right)\left[\frac{\Delta R^{n_{2}}(1)}{V^{n_{2}}(1)}-\frac{\Delta R^{n_{1}}(1)}{V^{n_{1}}(1)}\right] \Delta R^{n_{2}}=\Delta \underline{R}^{n^{\star}}-\Delta R^{n_{1}} \\
& =\left(\frac{1}{n_{1}+n_{2}}\right)^{1 / 2}\left(\Delta R^{n_{1}}(1)-\frac{V^{n_{1}}(1)}{V^{n^{\star}}} \Delta R^{n^{\star}}(1)\right)
\end{aligned}
$$


que corresponde ao conhecido teste exato de Fisher. Foi provado em (Leão e Ohashi)[16] que $\frac{M L R(1)}{\sqrt{\hat{\phi}_{n^{\star}}^{2}(1)}}$ converge em distribuição para $N(0,1)$ quando $n^{\star} \rightarrow \infty$, no qual

$$
\hat{\phi}_{n^{\star}}^{2}(1):=\frac{V^{n_{2}}(1)}{V^{n^{\star}}(1)} \hat{h}^{n_{1}}(1)\left[1-\hat{h}^{n_{1}}(1)\right]+\frac{V^{n_{1}}(1)}{V^{n^{\star}}(1)} \hat{h}^{n_{2}}(1)\left[1-\hat{h}^{n_{2}}(1)\right]
$$

Às vezes é necessário combinar resultados de diversas tabelas de contingência $2 \times 2$ em uma análise geral. Esta situação ocorre quando um experimento geral consiste em vários experimentos menores conduzidos em várias situações. Neste caso, os dados são resumidos em diversas tabelas de contingência $2 \times 2$ obtidas à partir de experimentos independentes. Seja $k$ o número de tabelas e $\pi_{\ell}^{1}$ a probabilidade de uma observação na linha 1 ser classificada na coluna 1 , na $\ell$-ésima tabela de contingência, e $\pi_{\ell}^{2}$ a probabilidade correspondente da linha 2. Consideramos a hipótese nula $H_{0}: \pi_{\ell}^{1}=\pi_{\ell}^{2}$, para todo $\ell=1, \ldots, k-1$. Denotamos $M L R(\ell)$ o vetor de Log-rank Modificado para a $\ell$-ésima tabela e $\hat{\phi}_{n^{*}}^{2, \ell}(\ell)$ a variância associada. Assim, chegamos à estatística de Mantel-Haenszel para tabelas de contingência $2 \times 2$ da seguinte forma

$$
M H(1)=\frac{M L R\left(n^{\star}, 1\right)}{\sqrt{\hat{\phi}^{2}\left(n^{\star}, 1\right)}}
$$

Agora, a estatística de Log-rank Modificada é dada por

$$
M X^{2}\left(n^{\star}, 1\right)=\frac{\left(M L R\left(n^{\star}, 1\right)\right)^{2}}{\hat{\phi}^{2}\left(n^{\star}, 1\right)}
$$

Exemplo 3.2. (Teste de Homogeneidade em tabelas $J \times k$ )

Esta tabela pode ser utilizada para apresentar dados contidos em várias amostras. Temos J amostras, e em vez de cada amostra fornecer duas categorias, consideramos $k$ categorias. No entanto, estamos sob o modelo de estrutura com censura, no qual os dados observados são dadas por $\left\{\Delta R_{m}^{p}, \Delta R_{m}^{C, p}(\ell)\right\}$. Para cada categoria podemos associar a tabela (3.3).

\begin{tabular}{cccccc}
\hline & Pop 1 & $\ldots$ & Pop J & Total \\
\hline Número de Eventos & $\Delta R^{n_{1}}(\ell)$ & $\ldots$ & $\Delta R^{n_{J}}(\ell)$ & $\Delta R^{n^{\star}}(\ell)$ \\
Número de Eventos sob Risco & $V^{n_{1}}(\ell)$ & $\ldots$ & $V^{n_{J}}(\ell)$ & $V^{n^{\star}}(\ell)$ \\
\hline
\end{tabular}

Tabela 3.3: Resumo das observações para a $\ell$-ésima categoria.

Agora, vamos generalizar o exemplo (3.1) para tabelas de contingência $J \times k$. 
Segue de (3.12) e tomando o peso log-rank, ou seja, $u\left(n^{\star}, \ell\right)=1$ que

$$
\begin{aligned}
M L R^{q}\left(n^{\star}, \ell\right) & :=\sum_{q_{1} \neq q}\left(\frac{1}{n}\right)^{1 / 2}\left(\frac{V^{n_{q}}(\ell) V^{n_{q_{1}}}(\ell)}{V^{n^{\star}}(\ell)}\right)[\underbrace{\hat{h}^{n}}_{\frac{\Delta R^{n_{q_{1}(\ell)}}(\ell)}{h_{q_{1}}}\left(\hat{h}_{q_{1}}(\ell)\right.}-\underbrace{\hat{h}^{n_{q}}(\ell)}_{\frac{\Delta R^{n}(\ell)}{V n_{q}(\ell)}}]= \\
& =\left(\frac{1}{n}\right)^{1 / 2}\left[\Delta R^{n_{q}}(\ell)-\frac{V^{n_{q}}(\ell) \Delta R^{n^{\star}}(\ell)}{V^{n^{\star}}(\ell)}\right]
\end{aligned}
$$

Assim, obtemos a estatística de Log-rank Modificado

$$
M X^{2}\left(n^{\star}, k-1\right)=\sum_{\ell=1}^{k-1} M L R_{0}\left(n^{\star}, \ell\right)^{T} \hat{Q}_{0}\left(n^{\star}, \ell\right)^{-1} M L R_{0}\left(n^{\star}, \ell\right),
$$

Seja $\pi_{\ell}^{p}$ a probabilidade de um elemento selecionado aleatoriamente à partir da variável aleatória de interesse $W^{p}$ ser classificada na $\ell$-ésima categoria, para $p=1, \ldots, J$ e $\ell=1, \ldots, k$. A hipótese $H_{0}$ : $\pi_{\ell}^{1}=\cdots=\pi_{\ell}^{J}$ para todo $\ell$, afirma que a probabilidade de estar na categoria $\ell$ é o mesmo para todas as variáveis aleatórias de interesse $W^{p}$, que é equivalente a hipótese $H_{0}: h^{1}(\ell)=\cdots=h^{J}(\ell)$ para todo $\ell=1, \cdots, k-1$. Neste caso, podemos aplicar a estatística de Log-rank Ponderada $X^{2}\left(n^{\star}, k-1\right)$ e a estatística de Log-rank Modificada $M X^{2}\left(n^{\star}, k-1\right)$ para testarmos a hipótese nula $H_{0}$.

\subsection{A estatística de Cramér-von Mises}

Nesta seção, enunciamos a estatística de Cramér-von Mises proposta por Leão e Ohashi [16] para populações discretas na presença de censura. A seguir, definimos a primeira categoria observada nas amostras por

$$
d_{n^{\star}}^{l}=\inf \left\{\ell: \Delta R^{n^{\star}}(\ell)>0\right\}
$$

e assumimos que todos os processos ponderados tem a forma (3.3). Assim, $d_{n^{\star}}^{l} \rightarrow d^{l}$ em probabilidade quando $n^{\star} \rightarrow \infty$ em que,

$$
d^{l}:=\inf \left\{\ell: b_{1} h^{1}(\ell)+\cdots+b_{J} h^{J}(\ell)>0\right\}
$$

para $b_{p}=\lim _{n^{\star} \rightarrow \infty} \frac{n_{p}}{n}, p \in \mathcal{J}$ (ver Leão e Ohashi [16] pág.19).

Para testarmos a homogeneidade das populações discretas na presença de censura arbitrária com infinitas categorias, enunciamos uma versão da estatística de Cramér-von Mises proposto no trabalho de Leão e Ohashi [16] (os detalhes matemáticos podem ser vistos neste trabalho).

Primeiramente, consideramos $L R\left(n^{\star}, r\right) \operatorname{com} n^{\star} \in \mathbb{N}^{J}$ e $r \geq 1$ ponderado pela sequência $\hat{\phi}_{n^{\star}}=$ 
$\left\{\hat{\phi}_{1, n^{\star}}(r), \ldots, \hat{\phi}_{J, n^{\star}}(r): r=1,2,3, \cdots\right\}$ no espaço de Hilbert ${ }^{1} \ell^{2}(\mathbb{N})$, obtendo

$$
G L R_{q}\left(n^{\star}, \hat{\phi}_{n^{\star}}, r\right)=\hat{\phi}_{q, n^{\star}}(r) L R_{q}\left(n^{\star}, r\right) ; \quad q=1, \ldots, J-1
$$

Assim, temos que

$$
\begin{aligned}
G L R\left(n^{\star}, \hat{\phi}_{n^{\star}}, r\right)= & \left(\hat{\phi}_{1, n^{\star}}(r) L R_{1}\left(n^{\star}, r\right), \ldots, \hat{\phi}_{J-1, n^{\star}}(r) L R_{J-1}\left(n^{\star}, r\right)\right)^{T} \\
& \stackrel{(3.15)}{=}\left(G L R_{1}\left(n^{\star}, \hat{\phi}_{n^{\star}}, r\right), \ldots, G L R_{J-1}\left(n^{\star}, \hat{\phi}_{n^{\star}}, r\right)\right)^{T}
\end{aligned}
$$

para $r \geq 1$ e $n^{\star} \in \mathbb{N}^{J}$.

Construímos agora $M_{0}(\ell):=\operatorname{diag}\left(\phi_{1}(\ell), \ldots, \phi_{J-1}(\ell)\right)$ e o operador de covariância, que é o mesmo definido em (3.6), porém, usamos a notação $\Gamma_{0}$ em vez de $\Gamma$ na forma quadrática. Denotamos o operador linear $\mathcal{Y}_{0}\left(d^{l}, d^{u}\right): \ell^{2} \rightarrow \ell^{2}$ de modo que $(J-1) k\left(d^{l}, i\right)$-ésima coordenada de $\mathcal{Y}_{0}\left(d^{l}, d^{u}\right) a \doteq$ $Y_{0}\left(d^{l}, i\right)\left(a_{1}, \ldots, a_{(J-1) k\left(d^{l}, i\right)}\right)$ para $a \in \ell^{2}$ e $k\left(d^{l}, i\right)=i-d^{l}+1 ; i \geq d^{l}$. Assim, introduzimos o conjunto $L\left(d_{n^{\star}}^{l}, d_{n^{\star}}^{u}\right)=\left\{d_{n^{\star}}^{l} \leq \ell \leq d_{n^{\star}}^{u}: \Delta R^{n^{\star}}(\ell)>0\right\}$ das categorias observáveis e $L\left(n^{\star}\right)$ a cardinalidade. Para um determinado $n^{\star} \in \mathbb{N}^{J}$ e $a \in \ell^{2}$, definimos

$$
\hat{\mathcal{Y}}_{0}\left(d_{n^{\star}}^{l}, d_{n^{\star}}^{u}\right) a
$$

uma sequência real em que a $(J-1) L\left(n^{\star}\right)$-ésima coordenada é obtida por

$$
\hat{Y}_{0}\left(d_{n^{\star}}^{l}, d_{n^{\star}}^{u}\right)\left(a_{1} \ldots, a_{(J-1) L\left(n^{\star}\right)}\right) ;
$$

e $\hat{Y}_{0}\left(d_{n^{\star}}^{l}, d_{n^{\star}}^{u}\right)$ é o operador aleatório auto-adjunto definido pela seguinte forma quadrática no espaço $\mathbb{R}^{(J-1) L\left(n^{\star}\right)}$. Assim, obtemos o seguinte produto interno

$$
\begin{aligned}
\left\langle\hat{\mathcal{Y}}_{0}\left(d_{n^{\star}}^{l}, d_{n^{\star}}^{u}\right) a, a\right\rangle & =\sum_{j \in L\left(d_{n^{\star}}^{l}, d_{n^{\star}}^{u}\right)}\left\langle\hat{M}_{0}(j) \hat{\Gamma}_{0}(j) \hat{M}_{0}(j) a_{j}, a_{j}\right\rangle_{\mathbb{R}^{J-1}}+ \\
& +\sum_{\left\{\ell<j: \ell, j \in L\left(d_{n^{\star}}^{l}, d_{n^{\star}}^{u}\right)\right\}}\left\langle\hat{M}_{0}(\ell) \hat{\Gamma}_{0}(\ell) \hat{M}_{0}(j) a_{\ell}, a_{j}\right\rangle_{\mathbb{R}^{J-1}}+ \\
& +\sum_{\left\{j<\ell: \ell, j \in L\left(d_{n^{\star}}^{l}, d_{n^{\star}}^{u}\right)\right\}}\left\langle\hat{M}_{0}(\ell) \hat{\Gamma}_{0}(j) \hat{M}_{0}(j) a_{\ell}, a_{j}\right\rangle_{\mathbb{R}^{J-1}}
\end{aligned}
$$

em que $\hat{M}_{0}(\cdot):=\operatorname{diag}\left(\hat{\phi}_{1, n^{\star}}(\cdot), \ldots, \hat{\phi}_{J-1, n^{\star}}(\cdot)\right)$ e $a \in \mathbb{R}^{(J-1) L\left(n^{\star}\right)}$.

Portanto, $\hat{\mathcal{Y}}_{0}\left(d_{n^{\star}}^{l}, d_{n^{\star}}^{u}\right): \ell^{2} \rightarrow \ell^{2}$ é uma sequência bem definida de operadores aleatórios auto-adjuntos

Definição 3.2. Dizemos que H é um espaço de Hilbert se $H$ for um espaço vetorial com produto interno que é um espaço de Banach com a norma derivada do produto interno. 
de posto finito.

Por fim, definimos a estatística de Cramér-von Mises associadas aos modelos discretos na presença de censura da seguinte forma

$$
C V M\left(n^{\star}, d_{n^{\star}}^{l}, d_{n^{\star}}^{u}\right):=\left\|G L R\left(n^{\star}, \hat{\phi}_{n^{\star}}, d_{n^{\star}}^{l}, d_{n^{\star}}^{u}\right)\right\|_{\ell^{2}}^{2} ; n^{\star} \in \mathbb{N}^{J-1} .
$$

Vamos enunciar dois teoremas do artigo de Leão e Ohashi[16], que são resultados fundamentais para a estatística de Cramér-von Mises.

Teorema 3.3.1. Suponha que os pressupostos (M1, M2, M3', M4') e (H1') (Leão e Ohashi [16]) são satisfeitos e seja $1 \leq d^{l} \leq d^{u}<\infty$ em que (S1-S2) também são. Então o limite $\lim _{n^{\star} \rightarrow \infty} G L R\left(n^{\star}, \hat{\phi}_{n^{\star}}, d_{n^{\star}}^{l}, d_{n^{\star}}^{u}\right)$ é uma medida de Gauss com média zero em $\ell^{2}$ com o operador covariância $\hat{\mathcal{Y}}\left(d_{n^{\star}}^{l}, d_{n^{\star}}^{u}\right)$ em $\ell^{2}$. Em particular,

$$
\left\|G L R\left(n^{\star}, \hat{\phi}_{n^{\star}}, d_{n^{\star}}^{l}, d_{n^{\star}}^{u}\right)\right\|_{\ell^{2}}^{2} \rightarrow \sum_{s=1}^{\infty} \sum_{q=1}^{J-1} \lambda_{s q} \chi_{s q}^{2} \quad \text { em distribuição quando } n^{\star} \rightarrow \infty
$$

em que $\left\{\lambda_{s q} ; s \geq 1, q=1, \ldots, J-1\right\}$ são os auto-valores do operador de covariância $\mathcal{Y}_{0}\left(d^{l}, d^{u}\right) e$ $\left\{\chi_{s q}^{2}, s \geq 1, q=1, \ldots, J\right\}$ é um subconjunto de variáveis aleatórias i.i.d. Qui-Quadrado com um grau de liberdade

Assim, como consequência do teorema (3.3.1) e da proposição (2.1.1), obtemos o seguinte resultado.

Teorema 3.3.2. Assumimos que $U$ pertence a classe $\mathcal{K}$ e satisfaz a condição de proporcionalidade do tamanho da amostra da proposição (2.1.1) e seja $\left(d^{l}, d^{u}, d_{n^{\star}}^{l}, d_{n^{\star}}^{l}\right)$ as categorias dos tempos de parada. Então, sob $H_{0}$

$$
C V M\left(n^{\star}, d_{n^{\star}}^{l}, d_{n^{\star}}^{u}\right) \rightarrow \sum_{s=1}^{\infty} \sum_{q=1}^{J-1} \lambda_{s q} \chi_{s q}^{2} \quad \text { fracamente quando } n^{\star} \rightarrow \infty
$$

em que $\left\{\lambda_{s q} ; s \geq 1, q=1, \ldots, J-1\right\}$ são os auto-valores do operador de covariância $\mathcal{Y}_{0}\left(d^{l}, d^{u}\right)$. Em particular, se $X^{q}$ é quadrado integrável para todo $q \in \mathcal{J}$ então

$$
\Lambda\left(n^{\star}\right) \doteq \sum_{s=1}^{L\left(n^{\star}\right)} \sum_{q=1}^{J-1} \hat{\lambda}_{s q} \chi_{s q}^{2} \mathbb{1}_{\left\{A\left(n^{\star}\right)\right\}} \rightarrow \sum_{s=1}^{\infty} \sum_{q=1}^{J-1} \lambda_{s q} \chi_{s q}^{2} \quad \text { em distribuição quando } n^{\star} \rightarrow \infty
$$

em que $\left\{\hat{\lambda}_{s q} ; 1 \leq s \leq L\left(n^{\star}\right), q=1, \ldots, J-1\right\}$ são os auto-valores aleatórios do estimador do operador de covariância $\hat{\mathcal{Y}}_{0}\left(d^{l}, d^{u}\right)$, e $A\left(n^{\star}\right) \doteq\left\{\hat{\mathcal{Y}}_{0}\left(d^{l}, d^{u}\right)\right.$, é não negativo $\}$, desde que $P\left[A\left(n^{\star}\right)\right] \rightarrow 1$ quando $n^{\star} \rightarrow \infty$. 
Notamos que à partir de (3.17), o p-valor para o teste de hipótese $H_{0}$ é dada por

$$
P\left[\Lambda\left(n^{\star}\right)>C V M\left(n^{\star}, d_{n^{\star}}^{l}, d_{n^{\star}}^{u}\right) \mid H_{0}\right]
$$

com $\Lambda\left(n^{\star}\right)$ soma ponderada de variáveis aleatórias Qui-Quadrado independentes. Para calcularmos o pvalor usamos um dos algoritmos descritos na literatura, como por exemplo (Robert B. Davies [4]). Apresentadas as três estatísticas a serem utilizadas neste trabalho, a seguir vamos apresentar um algoritmo para descrever os passos dos testes.

1. Classificamos $\left\{\left(X_{1}^{1}, \delta_{1}^{1}\right), \ldots,\left(X_{n_{J}}^{J}, \delta_{n_{J}}^{J}\right)\right\}$ da menor até a maior categoria;

2. Calculamos o processo de risco e o estimador de Kaplan-Meier como nas equações (2.10) e (2.11), respectivamente. Seja $\mathcal{I}=\{1, \ldots, I\}$ é o conjunto das categorias observáveis, em que $I$ é definido por (3.2) e 1 corresponde à posição da primeira categoria observada $\left(d_{n^{\star}}^{l}\right)$. Note que a classificação definida por $\mathcal{I}$ corresponde ao conjunto aleatório $L\left(d_{n^{\star}}^{l}, d_{n^{\star}}^{u}\right)$ das categorias observáveis definidas no artigo de Leão e Ohashi [16];

3. Escolhemos o processo de ponderação $u\left(n^{\star}, \cdot\right)$ de acordo com a classe $\mathcal{K}$ e calculamos $\hat{\Gamma}\left(n^{\star}, k\right)$ por meio de (3.6) e (3.7), para cada $k \in \mathcal{I}$; Obs: para a escolha do processo de ponderação tomamos algum processo definido na literatura (ver tabela (3.1))

4. Em seguida, calculamos as estatísticas lineares $\left\{L R_{q}\left(n^{\star}, \cdot\right): q=1, \ldots, J-1\right\}$ de acordo com (3.5). Assim temos que as estatísticas de Cramér-von Mises, Log-rank Ponderado e a de Log-rank Modificado são respectivamente dadas por

$$
\begin{aligned}
& \text { - } C V M\left(n^{\star}, 1, I\right)=\sum_{k=1}^{I} \sum_{q=1}^{J-1} \hat{\phi}_{q, n^{\star}}^{2}(k) L R_{q}^{2}\left(n^{\star}, k\right) ; \quad n^{\star} \in \mathbb{N}^{J}, \\
& \text { em que } \hat{\phi}_{q, n^{\star}}^{2}(\cdot) \text { é obtido por (3.7); } \\
& \text { - } X^{2}\left(n^{\star}, 1, I\right)=L R_{0}\left(n^{\star}, d_{n^{\star}}^{u}\right)^{T} \hat{\Gamma}_{0}\left(n^{\star}, d_{n^{\star}}^{u}\right)^{-1} L R_{0}\left(n^{\star}, d_{n^{\star}}^{u}\right) ; \\
& \text { - } M X^{2}\left(n^{\star}, 1, I\right)=\sum_{\ell=1}^{k-1} M L R_{0}\left(n^{\star}, \ell\right)^{T} \hat{Q}_{0}\left(n^{\star}, \ell\right)^{-1} M L R_{0}\left(n^{\star}, \ell\right), \quad \mathcal{K}(\text { finito } \subseteq \mathbb{N} .
\end{aligned}
$$

Definimos $M_{0}(\ell):=\operatorname{diag}\left(\phi_{1}(\ell), \ldots, \phi_{J-1}(\ell)\right)$ e o operador de covariância, que é o mesmo definido em (3.6), porém, usamos a notação $M_{0}, Q_{0}$ e $\Gamma_{0}$ em vez de $M, Q$ e $\Gamma$ na forma quadrática.

5. Para calcular o $P$-valor do teste de hipótese de CVM, calculamos a matriz simétrica $I(J-1)$ dimensional $\hat{\mathcal{Y}}_{0}(1, I)$, que é o operador linear no qual é composto por blocos de dimensão $J-1$ como a seguir

$$
\left[\hat{\mathcal{Y}}_{0}(1, I)\right]_{\ell j}:= \begin{cases}\hat{M}_{0}(j) \hat{\Gamma}_{0}\left(n^{\star}, j\right) \hat{M}_{0}(j) ; & \text { se } j=\ell \\ \hat{M}_{0}(\ell) \hat{\Gamma}_{0}\left(n^{\star}, \ell\right) \hat{M}_{0}(j) ; & \text { se } j>\ell \\ \hat{M}_{0}(\ell) \hat{\Gamma}_{0}\left(n^{\star}, j\right) \hat{M}_{0}(j) ; & \text { se } j<\ell\end{cases}
$$


para $j, \ell \in \mathcal{I}$. Com a matriz simétrica $\hat{\mathcal{Y}}_{0}(1, I)$ em mãos, calculamos o $P$-valor, sendo que para este, existem muitos algoritmos para calculá-lo. Para mais detalhes veja Robert B. Davies [4].

Um breve resumo do que foi dito até agora é que se $\pi_{\ell}^{p}$ é a probabilidade de um elemento selecionado aleatoriamente à partir da variável aleatória de interesse $W^{p}$ ser classificada na $\ell$-ésima categoria, para $p=1, \ldots, J$ e $\ell=1, \ldots, k$. A hipótese $H_{0}: \pi_{\ell}^{1}=\cdots=\pi_{\ell}^{J}$ para todo $\ell$, afirma que a probabilidade de estar na categoria $\ell$ é o mesmo para todas as variáveis aleatórias de interesse $W^{p}$, que é equivalente a hipótese $H_{0}: h^{1}(\ell)=\cdots=h^{J}(\ell)$ para todo $\ell=1, \cdots, K-1$. Para este tipo de problema aplicamos as classes estatísticas de Log-rank Ponderado $X^{2}\left(n^{\star}, d_{n^{\star}}^{u}\right)$, Log-rank Modificado $M X^{2}\left(n^{\star}, d_{n^{\star}}^{u}\right)$ (caso $\mathcal{K}$ finito) e de Cramér-von Mises $C V M\left(n^{\star}, d_{n^{\star}}^{\ell}, d_{n^{\star}}^{u}\right)$ para testarmos as hipóteses. Obtido o algoritmo, no capítulo seguinte vamos estudar o comportamento das estatísticas apresentadas neste capítulo. 


\section{Capítulo 4}

\section{Estudo de Simulação}

Neste capítulo, avaliamos através de um estudo de simulação o comportamento das estatísticas propostas neste trabalho, que são testes que comparam diferenças ponderadas entre as funções de intensidade das amostras. O intuito de ponderarmos estas diferenças está em dar mais ênfase em certas partes da função intensidade. Neste capítulo, utilizamos os processos de ponderação descritos na Tabela (3.1).

A principal motivação deste trabalho, é analisar dados em que temos cruzamento das funções intensidade. Através do estudo de simulação comparamos as estatísticas propostas neste trabalho com as estatísticas de Renyi e Cramér-von Mises para dados contínuos (CCVM), que são baseadas na avaliação do supremo da diferença entre as funções intensidade e na diferença ao quadrado, integrado entre as duas funções de sobrevivência empíricas respectivamente. Estes testes, segundo Klein e Moeschberger (1997) [15], têm alto poder para detectar alternativas em que as funções intensidade cruzam e são extensões do teste de Kolmogorov-Smirnov usual para comparação de duas amostras na ausência de censura. O principal problema em dados com cruzamento nas funções de intensidade consiste no fato de que as diferenças iniciais em favor de um tratamento são cancelados em favor do outro tratamento. Segundo Klein e Moeschberger (1997) [15], a estatística de Log-rank têm poder ótimo para detectar alternativas em que as funções intensidade das $J$ populações são proporcionais, enquanto que o poder é baixo para funções intensidade não proporcionais, principalmente quando ocorrem cruzamentos.

No estudo de simulação analisamos o efeito do tamanho da amostra, as proporções de dados com censura, número de populações, níveis de cruzamento das funções de intensidade e truncamento em dados contínuos. No primeiro estudo avaliamos o comportamento das estatística em espaços amostrais finitos, na sequência, em espaços infinitos enumeráveis. 
Para o caso de espaços amostrais finitos $\mathcal{K}$, avaliamos diversas situações como funções intensidade proporcionais, não proporcionais com um e dois cruzamentos das funções de intensidade e sob a hipótese nula $\left(H_{0}\right)$. Para o caso de espaços amostrais infinitos enumeráveis $\mathcal{K} \subseteq \mathbb{N}$, avaliamos a função poder do teste para dados em que há um cruzamento das funções de intensidade, para isto tomamos três situações de cruzamentos (início, meio e fim das funções de intensidade). Por fim, avaliamos os testes em dados truncados ou arredondados e comparamos com as estatísticas de Renyi e CCVM citadas em [15]. Para ilustrar todos os passos utilizados neste capítulo, apresentamos um fluxograma (4.1) do estudo de simulação. Em todo capítulo realizamos as simulações usando o software estatístico R ([19]) versão 2.13.1. A seguir, iniciamos os estudos com espaços amostrais finitos.

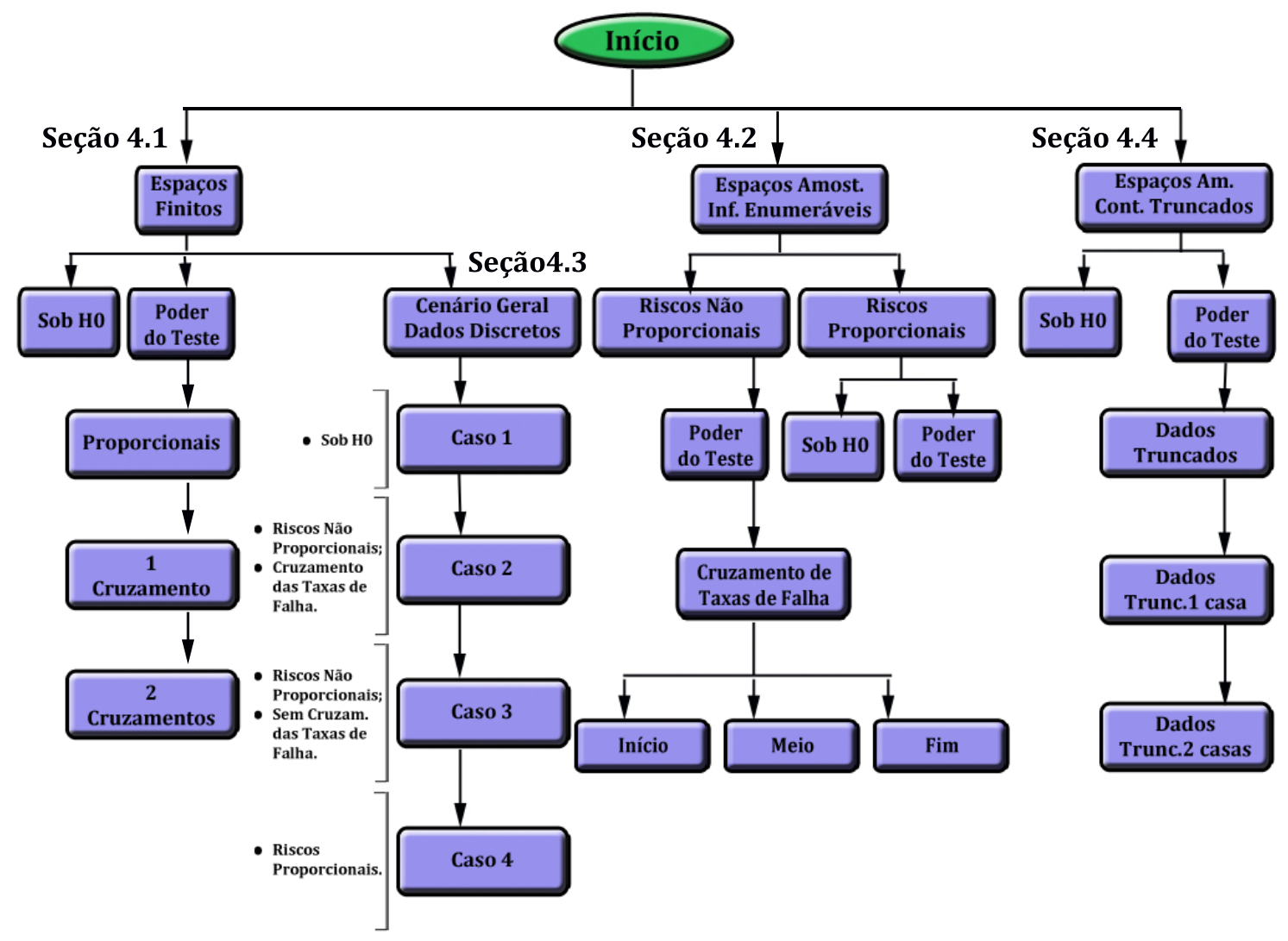

Figura 4.1: Fluxograma da Simulação. 


\subsection{Simulação para espaços amostrais finitos}

Nesta seção, avaliamos as estatísticas Log-rank Ponderado, Cramér-von Mises e Log-rank Modificado contra o clássico teste Qui-Quadrado de homogeneidade, aplicados em populações com espaço amostral finito $\mathcal{K}$ e sem censura.

No primeiro experimento, avaliamos sob a hipótese nula, em seguida avaliamos o poder do teste para três situações. A primeira delas é quando fixamos o tamanho de amostra, para na sequência incrementar valores de $\delta$ na função intensidade da população de controle. No segundo experimento, avaliamos o poder do teste para um dado valor fixado de $\delta$ para em seguida, variarmos o tamanho da amostra até um valor suficientemente grande. Para o terceiro experimento, avaliamos o cruzamento das funções de intensidade, primeiro com um cruzamento, em seguida com dois cruzamentos.

\subsubsection{Simulação sob a hipótese nula}

Aqui, tomamos duas amostras aleatórias com distribuição Multinomial com $W_{1}, W_{2} \sim M\left(n, p_{1}, p_{2}, p_{3}\right)$ em que $n=$ TA e $p_{1}=0,3, p_{2}=0,42$ e $p_{3}=0,28$. Para os estudos de simulação usamos todos os processos de ponderação definidos na tabela (3.1) e não censuramos os dados (pois vamos comparar com o teste Qui-Quadrado). Os tamanhos de amostras escolhidos para o experimento foram de TA=50, 75, 100, 125 e 200. A seguir, apresentamos o algoritmo para a simulação sob a hipótese nula:

1. Fixamos um nível de significância $\alpha=0,05$;

2. Geramos populações de tamanho TA ${ }^{1}$ com distribuição Multinomial com parâmetros $\left(n, p_{1}, p_{2}, p_{3}\right)$ dado que a hipótese nula é verdadeira;

3. Escolhemos uma das funções de ponderação descrita na tabela (3.1);

4. Realizamos o teste de hipótese usando valor crítico ao nível de significância $\alpha$;

5. Verificamos se cometeu o erro do tipo I, isto é, se a hipótese nula foi rejeitada;

$$
y_{m}= \begin{cases}1, & \text { se } H_{0} \text { é rejeitada ao nível de significância } \alpha \\ 0, & \text { caso contrário }\end{cases}
$$

6. Repetimos as etapas 2 a $4 m=10000$ vezes;

7. Estimamos a probabilidade de cometer o erro do tipo I da seguinte forma:

$$
\hat{\alpha}=\frac{1}{m} \sum_{k=1}^{m} y_{k}
$$

\footnotetext{
${ }^{1}$ Tamanho da Amostra
} 
À partir dos resultados obtidos na tabela (4.1) e figura (4.2) notamos que as estimativas da probabilidade de cometermos o erro tipo I para as todos os testes estatísticos estão muito próximas da probabilidade nominal $(\alpha)$. Porém, o teste qui-quadrado apresenta uma convergência ligeiramente melhor.

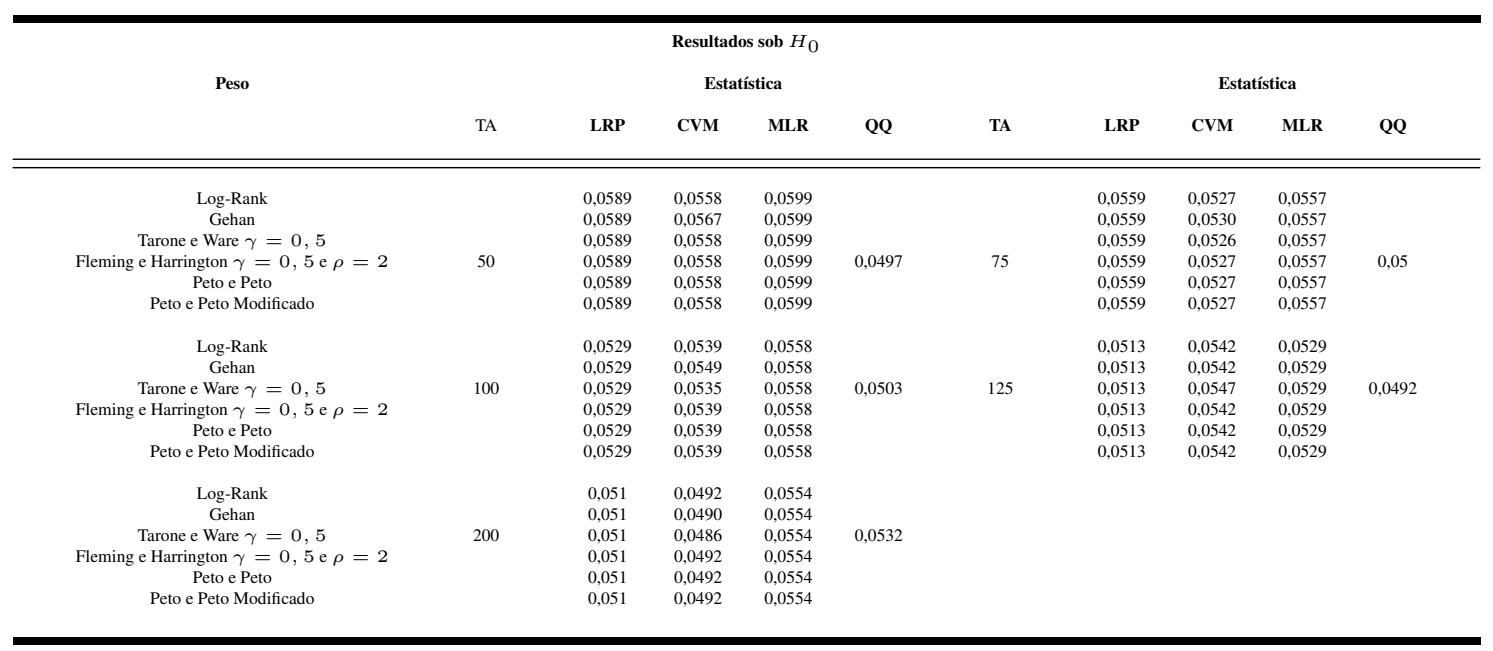

Tabela 4.1: Simulação sob a hipótese nula $H_{0}$ para espaços finitos.
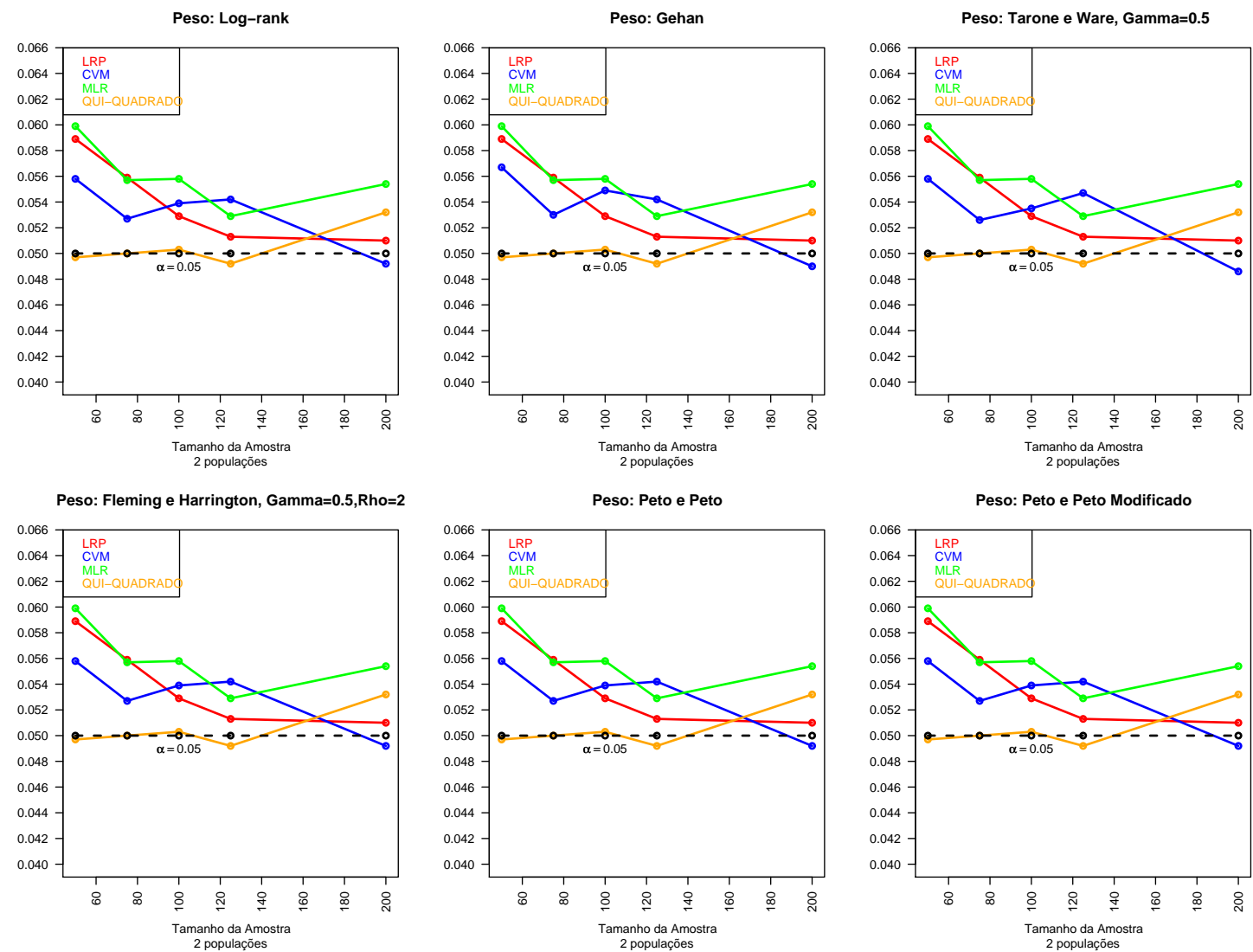

Figura 4.2: Gráfico da simulação sob a hipótese nula $H_{0}$ (sem censura) e espaço finito. 


\subsubsection{Simulação do poder do teste para funções de intensidade proporcionais}

Nesta seção, realizamos um estudo de simulação para avaliarmos o poder do teste quando a hipótese alternativa é incrementada por $\delta$ e o tamanho da amostra está fixo. Neste experimento, tomamos amostras aleatórias com distribuição Multinomial com parâmetros $\left(T A, p_{1}, p_{2}, p_{3}\right)$. Aqui, fixamos o tamanho da amostra em 500 e definimos a população de tratamento com função de intensidade $h^{n_{1}}=(0,1+\delta \quad 0,2+$ $\delta$ 1) e as populações controle com funções de intensidade $h^{n_{2}}=\cdots=h^{n_{J}}=(0,1 \quad 0,21)$. O número de populações adotadas no experimento são de 2, 3 e 5 populações e todas as funções de ponderação descritas na tabela (3.1). Além disso, os valores de $\delta$ escolhidos para a simulação são $0,0,01,0,015,0,02,0,022$, $0,025,0,03,0,035,0,04,0,05,0,055,0,06,0,065,0,07,0,08,0,09$ e 0,1 . A seguir apresentamos os passos do algoritmo.

1. Escolhemos $\delta$ e fixamos o nível de significância $\alpha=0,05$;

2. Fixamos o tamanho da amostra em 500 e definimos $J(2,3$ e 5$)$ populações com distribuição Multinomial:

- $X^{(1)}, \ldots, X^{(J)} \sim M\left(T A, p_{1}, p_{2}, p_{3}\right)$

- A função de intensidade para a população de controle $h^{n_{1}}=\left(\begin{array}{llll}0,1+\delta & 0,2+\delta \quad 1\end{array}\right)$;

- A função de intensidade para as populações de tratamento $h^{n_{2}}=\cdots=h^{n_{J}}=\left(\begin{array}{lll}0,1 & 0,2 & 1\end{array}\right)$;

- À partir das funções de intensidade, calculamos as probabilidades $p_{1}, p_{2}$ e $p_{3}$ usando a equação (2.5), ou seja, $\pi_{i}=P[W=i]=h(i) \prod_{\ell=1}^{i-1}[1-h(\ell)]$ dado que a hipótese nula é falsa;

- Escolhemos uma das funções de ponderação descrita na tabela (3.1);

3. Realizamos o teste de hipótese usando valor crítico ao nível de significância $\alpha$;

4. Para estimarmos a função poder tomamos

$$
y_{m}= \begin{cases}1, & \text { se } H_{0} \text { foi rejeitada ao nível de significância } \alpha \\ 0, & \text { caso contrário }\end{cases}
$$

5. Repetimos as etapas 2 a $4 m=10000$ vezes;

6. Estimamos o poder do teste na seguinte forma:

$$
\text { Poder }=\frac{1}{m} \sum_{k=1}^{m} y_{k}
$$




\begin{tabular}{|c|c|c|c|c|c|c|c|c|c|c|c|c|c|c|c|}
\hline \multirow[b]{2}{*}{$\delta$} & \multirow[b]{2}{*}{ Peso } & \multicolumn{4}{|c|}{ Estatística } & \multicolumn{5}{|c|}{ Estatística } & \multicolumn{5}{|c|}{ Estatística } \\
\hline & & LRP & CVM & MLR & QQ & Peso & LRP & CVM & MLR & QQ & Peso & LRP & CVM & MLR & QQ \\
\hline 0 & & 0,0494 & 0,0511 & 0,0505 & 0,0513 & & 0,0502 & 0,0512 & 0,0505 & 0,0501 & & 0,0502 & 0,0520 & 0,0505 & 0,0498 \\
\hline 0,01 & & 0,0929 & 0,0943 & 0,0804 & 0,0792 & & 0,0943 & 0,0954 & 0,0804 & 0,0791 & & 0,0939 & 0,0963 & 0,0804 & 0,0793 \\
\hline 0,015 & & 0,1488 & 0,1545 & 0,1225 & 0,1221 & & 0,1516 & 0,1569 & 0,1225 & 0,1222 & & 0,1507 & 0,1571 & 0,1225 & 0,1219 \\
\hline 0,02 & & 0,2292 & 0,2374 & 0,1842 & 0,183 & & 0,2336 & 0,2397 & 0,1842 & 0,1831 & & 0,2311 & 0,2404 & 0,1842 & 0,1825 \\
\hline 0,022 & & 0,269 & 0,2797 & 0,2206 & 0,219 & & 0,2746 & 0,2828 & 0,2206 & 0,219 & & 0,2715 & 0,2807 & 0,2206 & 0,2185 \\
\hline 0,025 & & 0,3222 & 0,3374 & 0,2678 & 0,2661 & & 0,3302 & 0,3391 & 0,2678 & 0,2662 & & 0,3267 & 0,3386 & 0,2678 & 0,2659 \\
\hline 0,03 & & 0,4308 & 0,4462 & 0,3591 & 0,3573 & & 0,4398 & 0,4505 & 0,3591 & 0,3571 & & 0,4358 & 0,4487 & 0,3591 & 0,3573 \\
\hline 0,035 & & 0,5406 & 0,5604 & 0,4609 & 0,4612 & & 0,5503 & 0,5634 & 0,4609 & 0,4621 & & 0,546 & 0,5638 & 0,4609 & 0,4595 \\
\hline 0,04 & & 0,6455 & 0,6644 & 0,5691 & 0,5661 & & 0,6559 & 0,6695 & 0,5691 & 0,5662 & & 0,6515 & 0,6675 & 0,5691 & 0,5663 \\
\hline 0,045 & Log-rank & 0,7511 & 0,7712 & 0,6782 & 0,6761 & Gehan & 0,7617 & 0,7733 & 0,6782 & 0,6764 & Tarone e & 0,7573 & 0,7725 & 0,6782 & 0,6756 \\
\hline 0,05 & & 0,8299 & 0,8459 & 0,7691 & 0,7672 & & 0,8375 & 0,8443 & 0,7694 & 0,7673 & Ware & 0,8341 & 0,8455 & 0,7691 & 0,7674 \\
\hline 0,055 & & 0,8885 & 0,9003 & 0,8387 & 0,8374 & & 0,896 & 0,9011 & 0,8387 & 0,8372 & $\gamma=0,5$ & 0,8924 & 0,9022 & 0,8387 & 0,8372 \\
\hline 0,06 & & 0,9309 & 0,9403 & 0,8949 & 0,8941 & & 0,9365 & 0,9396 & 0,8949 & 0,8942 & & 0,9346 & 0,9403 & 0,8949 & 0,8936 \\
\hline 0,065 & & 0,9631 & 0,9695 & 0,9391 & 0,9382 & & 0,9663 & 0,9701 & 0,9392 & 0,9383 & & 0,9657 & 0,9703 & 0,9392 & 0,9384 \\
\hline 0,07 & & 0,9778 & 0,9823 & 0,9619 & 0,9621 & & 0,9797 & 0,9826 & 0,9619 & 0,9623 & & 0,9786 & 0,9826 & 0,9619 & 0,9618 \\
\hline 0,08 & & 0,9946 & 0,9961 & 0,9891 & 0,9892 & & 0,9955 & 0,9955 & 0,9892 & 0,9892 & & 0,9952 & 0,9963 & 0,9894 & 0,9889 \\
\hline 0,09 & & 0,9983 & 0,9986 & 0,9966 & 0,9971 & & 0,9985 & 0,9989 & 0,9966 & 0,9974 & & 0,9983 & 0,9988 & 0,9966 & 0,9966 \\
\hline 0,1 & & 1 & 0,9999 & 0,9996 & 1 & & 1 & 0,9997 & 0,9996 & 1 & & 1 & 0,9998 & 0,9996 & 0,9996 \\
\hline 0 & & 0,0494 & 0,0511 & 0,0505 & 0,0501 & & 0,0494 & 0,0511 & 0,0505 & 0,0503 & & 0,0494 & 0,0511 & 0,0505 & 0,0498 \\
\hline 0,01 & & 0,0929 & 0,0943 & 0,0804 & 0,0791 & & 0,0929 & 0,0943 & 0,0804 & 0,0792 & & 0,0929 & 0,0943 & 0,0804 & 0,0793 \\
\hline 0,015 & & 0,1488 & 0,1545 & 0,1225 & 0,1224 & & 0,1488 & 0,1545 & 0,1225 & 0,1222 & & 0,1488 & 0,1545 & 0,1225 & 0,1219 \\
\hline 0,02 & & 0,2292 & 0,2374 & 0,1842 & 0,1832 & & 0,2292 & 0,2374 & 0,1842 & 0,1831 & & 0,2292 & 0,2374 & 0,1842 & 0,1825 \\
\hline 0,022 & & 0,269 & 0,2797 & 0,2206 & 0,219 & & 0,269 & 0,2797 & 0,2206 & 0,219 & & 0,269 & 0,2797 & 0,2206 & 0,2185 \\
\hline 0,025 & & 0,3222 & 0,3374 & 0,2678 & 0,2661 & & 0,3222 & 0,3374 & 0,2678 & 0,2662 & & 0,3222 & 0,3374 & 0,2678 & 0,2659 \\
\hline 0,03 & & 0,4308 & 0,4462 & 0,3591 & 0,3571 & & 0,4308 & 0,4462 & 0,3591 & 0,3572 & & 0,4308 & 0,4462 & 0,3591 & 0,3573 \\
\hline 0,035 & & 0,5406 & 0,5604 & 0,4609 & 0,4634 & & 0,5406 & 0,5604 & 0,4609 & 0,4611 & & 0,5406 & 0,5604 & 0,4609 & 0,4595 \\
\hline 0,04 & & 0,6455 & 0,6644 & 0,5691 & 0,5663 & & 0,6455 & 0,6644 & 0,5691 & 0,5661 & & 0,6455 & 0,6644 & 0,5691 & 0,5663 \\
\hline 0,045 & Fleming e & 0,7511 & 0,7712 & 0,6782 & 0,6762 & Peto e & 0,7513 & 0,7711 & 0,6782 & 0,6761 & Peto e & 0,7513 & 0,7713 & 0,6782 & 0,6756 \\
\hline 0,05 & Harrington & 0,8299 & 0,8459 & 0,7695 & 0,7674 & Peto & 0,8299 & 0,8459 & 0,7694 & 0,7673 & Peto & 0,8299 & 0,8459 & 0,769 & 0,7674 \\
\hline 0,055 & $\gamma=0,5 \mathrm{e}$ & 0,8885 & 0,9003 & 0,8387 & 0,8371 & & 0,8885 & 0,9003 & 0,8387 & 0,8371 & Modificado & 0,8885 & 0,9003 & 0,8387 & 0,8372 \\
\hline 0,06 & $\begin{array}{l}\rho-0 \\
\rho=2\end{array}$ & 0,9309 & 0,9403 & 0,8949 & 0,8943 & & 0,9309 & 0,9403 & 0,8949 & 0,8943 & & 0,9309 & 0,9403 & 0,8949 & 0,8936 \\
\hline 0,065 & & 0,9631 & 0,9695 & 0,9394 & 0,9383 & & 0,9631 & 0,9695 & 0,9392 & 0,9381 & & 0,9631 & 0,9695 & 0,9391 & 0,9384 \\
\hline 0,07 & & 0,9778 & 0,9823 & 0,9619 & 0,9623 & & 0,9778 & 0,9823 & 0,9619 & 0,9621 & & 0,9778 & 0,9823 & 0,9619 & 0,9618 \\
\hline 0,08 & & 0,9946 & 0,9961 & 0,9892 & 0,9894 & & 0,9946 & 0,9961 & 0,9892 & 0,9894 & & 0,9946 & 0,9961 & 0,989 & 0,9889 \\
\hline 0,09 & & 0,9983 & 0,9986 & 0,9966 & 0,9971 & & 0,9983 & 0,9986 & 0,9966 & 0,9974 & & 0,9983 & 0,9986 & 0,9966 & 0,9966 \\
\hline 0,1 & & 1 & 0,9999 & 0,9996 & 1 & & 1 & 0,9999 & 0,9996 & 1 & & 1 & 0,9999 & 0,9996 & 0,9996 \\
\hline
\end{tabular}

Tabela 4.2: Função Poder do teste para 2 populações, tamanho de amostra 500 e sem censura.
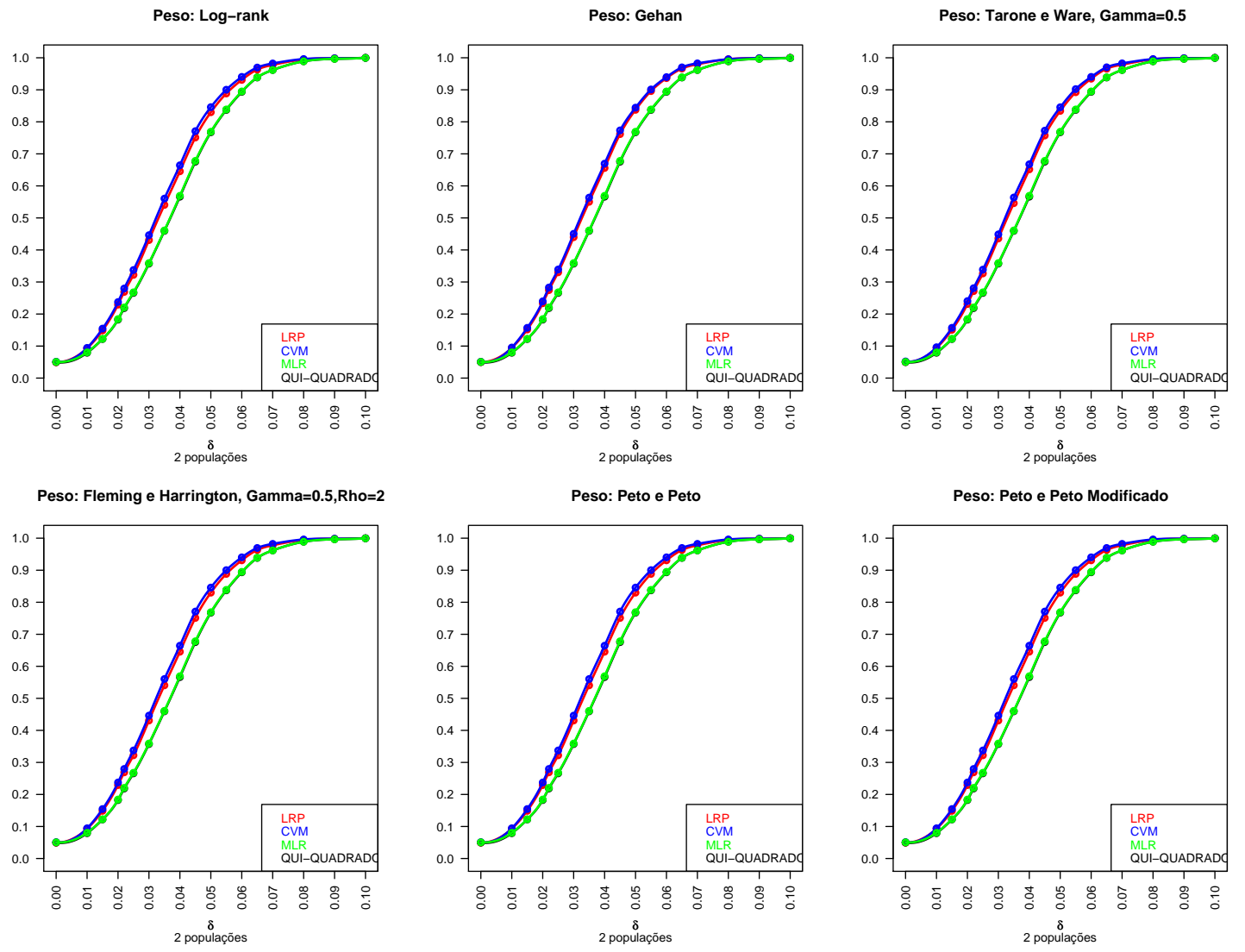

Figura 4.3: Gráfico da Função Poder do teste para 2 populações, tamanho de amostra 500 e sem censura. 


\begin{tabular}{|c|c|c|c|c|c|c|c|c|c|c|c|c|c|c|c|}
\hline \multirow[b]{2}{*}{$\delta$} & \multirow[b]{2}{*}{ Peso } & \multicolumn{4}{|c|}{ Estatística } & \multicolumn{5}{|c|}{ Estatística } & \multicolumn{5}{|c|}{ Estatística } \\
\hline & & LRP & CVM & MLR & QQ & Peso & LRP & CVM & MLR & QQ & Peso & LRP & CVM & MLR & QQ \\
\hline 0 & & 0,0487 & 0,0484 & 0,0538 & 0,0521 & & 0,0490 & 0,0486 & 0,0538 & 0,0522 & & 0,0484 & 0,0485 & 0,0538 & 0,0516 \\
\hline 0,01 & & 0,0918 & 0,1012 & 0,0841 & 0,0822 & & 0,0922 & 0,1002 & 0,0841 & 0,0821 & & 0,0921 & 0,1008 & 0,0841 & 0,0816 \\
\hline 0,015 & & 0,1414 & 0,1628 & 0,1161 & 0,1171 & & 0,1431 & 0,1634 & 0,1161 & 0,1171 & & 0,1421 & 0,1626 & 0,1161 & 0,1171 \\
\hline 0,02 & & 0,2235 & 0,2533 & 0,1786 & 0,1822 & & 0,2272 & 0,2556 & 0,1786 & 0,1822 & & 0,2247 & 0,2556 & 0,1786 & 0,1822 \\
\hline 0,022 & & 0,253 & 0,2922 & 0,2007 & 0,207 & & 0,2594 & 0,2969 & 0,2007 & 0,207 & & 0,2572 & 0,2921 & 0,2007 & 0,2068 \\
\hline 0,025 & & 0,3218 & 0,3644 & 0,2626 & 0,2672 & & 0,3258 & 0,3676 & 0,2626 & 0,2671 & & 0,3248 & 0,3665 & 0,2626 & 0,2672 \\
\hline 0,03 & & 0,4333 & 0,486 & 0,3559 & 0,3673 & & 0,4424 & 0,4912 & 0,3559 & 0,3671 & & 0,4391 & 0,4905 & 0,3559 & 0,3665 \\
\hline 0,035 & & 0,5593 & 0,6093 & 0,4707 & 0,4853 & & 0,5696 & 0,6182 & 0,4707 & 0,4854 & & 0,5644 & 0,6147 & 0,4707 & 0,4854 \\
\hline 0,04 & & 0,6773 & 0,7294 & 0,5931 & 0,6093 & & 0,6896 & 0,7321 & 0,5931 & 0,6094 & & 0,6845 & 0,7328 & 0,5931 & 0,6085 \\
\hline 0,045 & Log-rank & 0,7757 & 0,818 & 0,6956 & 0,7114 & Gehan & 0,7874 & 0,8212 & 0,6956 & 0,7114 & Tarone e & 0,7813 & 0,8195 & 0,6956 & 0,7114 \\
\hline 0,05 & & 0,8551 & 0,8876 & 0,7901 & 0,8051 & & 0,8643 & 0,8907 & 0,7901 & 0,8054 & Ware & 0,86 & 0,8889 & 0,7901 & 0,8054 \\
\hline 0,055 & & 0,9151 & 0,9343 & 0,8641 & 0,8772 & & 0,9226 & 0,9352 & 0,8641 & 0,8771 & $\gamma=0,5$ & 0,9183 & 0,9352 & 0,8641 & 0,8767 \\
\hline 0,06 & & 0,9542 & 0,9685 & 0,9189 & 0,9291 & & 0,9586 & 0,9693 & 0,9189 & 0,9293 & & 0,9563 & 0,9692 & 0,9189 & 0,9291 \\
\hline 0,065 & & 0,9744 & 0,9814 & 0,9537 & 0,9593 & & 0,9774 & 0,9824 & 0,9537 & 0,9593 & & 0,9764 & 0,9828 & 0,9537 & 0,9587 \\
\hline 0,07 & & 0,9887 & 0,9923 & 0,9754 & 0,9783 & & 0,9906 & 0,9924 & 0,9754 & 0,9784 & & 0,99 & 0,9929 & 0,9754 & 0,9783 \\
\hline 0,08 & & 0,9982 & 0,9991 & 0,9955 & 0,9973 & & 0,9986 & 0,9991 & 0,9955 & 0,9972 & & 0,9984 & 0,9991 & 0,9955 & 0,9965 \\
\hline 0,09 & & 0,9995 & 0,9998 & 0,999 & 1 & & 0,9996 & 0,9997 & 0,999 & 1 & & 0,9995 & 0,9998 & 0,999 & 0,9995 \\
\hline 0,1 & & 1 & 1 & 0,9997 & 1 & & 1 & 1 & 0,9997 & 1 & & 1 & 1 & 0,9997 & 0,9997 \\
\hline 0 & & 0,0487 & 0,0484 & 0,0538 & 0,0521 & & 0,0487 & 0,0484 & 0,0538 & 0,0523 & & 0,0487 & 0,0484 & 0,0538 & 0,0516 \\
\hline 0,01 & & 0,0918 & 0,1011 & 0,0841 & 0,0822 & & 0,0918 & 0,101 & 0,0841 & 0,0823 & & 0,0918 & 0,1012 & 0,0841 & 0,0816 \\
\hline 0,015 & & 0,1414 & 0,1628 & 0,1161 & 0,1172 & & 0,1414 & 0,1628 & 0,1161 & 0,1171 & & 0,1414 & 0,1628 & 0,1161 & 0,1171 \\
\hline 0,02 & & 0,2235 & 0,2533 & 0,1786 & 0,1823 & & 0,2235 & 0,2533 & 0,1786 & 0,1823 & & 0,2235 & 0,2533 & 0,1786 & 0,1822 \\
\hline 0,022 & & 0,253 & 0,2922 & 0,2007 & 0,207 & & 0,253 & 0,2922 & 0,2007 & 0,207 & & 0,253 & 0,2922 & 0,2007 & 0,2068 \\
\hline 0,025 & & 0,3218 & 0,3644 & 0,2626 & 0,2673 & & 0,3218 & 0,3644 & 0,2626 & 0,2673 & & 0,3218 & 0,3644 & 0,2626 & 0,2672 \\
\hline 0,03 & & 0,4332 & 0,4862 & 0,3559 & 0,3672 & & 0,4332 & 0,4862 & 0,3559 & 0,3672 & & 0,4332 & 0,4862 & 0,3559 & 0,3665 \\
\hline 0,035 & & 0,5593 & 0,6093 & 0,4707 & 0,4853 & & 0,5593 & 0,6093 & 0,4707 & 0,4852 & & 0,5593 & 0,6093 & 0,4707 & 0,4854 \\
\hline 0,04 & & 0,6772 & 0,7294 & 0,5931 & 0,6092 & & 0,6773 & 0,7294 & 0,5931 & 0,6091 & & 0,677 & 0,7294 & 0,5931 & 0,6085 \\
\hline 0,045 & Fleming e & 0,7757 & 0,818 & 0,6956 & 0,7111 & Peto e & 0,7757 & 0,818 & 0,6956 & 0,7111 & Peto e & 0,7757 & 0,818 & 0,6956 & 0,7112 \\
\hline 0,05 & Harrington & 0,8552 & 0,8876 & 0,7901 & 0,8052 & Peto & 0,8553 & 0,8876 & 0,7901 & 0,8051 & Peto & 0,8552 & 0,8876 & 0,7901 & 0,8054 \\
\hline 0,055 & $\gamma=0,5 \mathrm{e}$ & 0,9151 & 0,9343 & 0,8641 & 0,8772 & & 0,9151 & 0,9343 & 0,8641 & 0,8771 & Modificado & 0,9151 & 0,9343 & 0,8641 & 0,8767 \\
\hline 0,06 & $\rho=2$ & 0,9542 & 0,9685 & 0,9189 & 0,9291 & & 0,9542 & 0,9685 & 0,9189 & 0,9292 & & 0,9542 & 0,9685 & 0,9189 & 0,9291 \\
\hline 0,065 & & 0,9744 & 0,9814 & 0,9537 & 0,9592 & & 0,9744 & 0,9814 & 0,9537 & 0,9593 & & 0,9744 & 0,9814 & 0,9537 & 0,9587 \\
\hline 0,07 & & 0,9887 & 0,9923 & 0,9754 & 0,9781 & & 0,9887 & 0,9923 & 0,9754 & 0,9782 & & 0,9887 & 0,9923 & 0,9754 & 0,9783 \\
\hline 0,08 & & 0,9982 & 0,9991 & 0,9955 & 0,9972 & & 0,9982 & 0,9991 & 0,9955 & 0,9971 & & 0,9982 & 0,9991 & 0,9955 & 0,9965 \\
\hline 0,09 & & 0,9995 & 0,9998 & 0,9998 & 1 & & 0,9995 & 0,9998 & 0,9997 & 1 & & 0,9995 & 0,9998 & 0,999 & 0,9995 \\
\hline 0,1 & & 1 & 1 & 0,9997 & 1 & & 1 & 1 & 0,9997 & 1 & & 1 & 1 & 0,9997 & 0,9997 \\
\hline
\end{tabular}

Tabela 4.3: Função Poder do teste para 3 populações, tamanho de amostra 500 e sem censura.

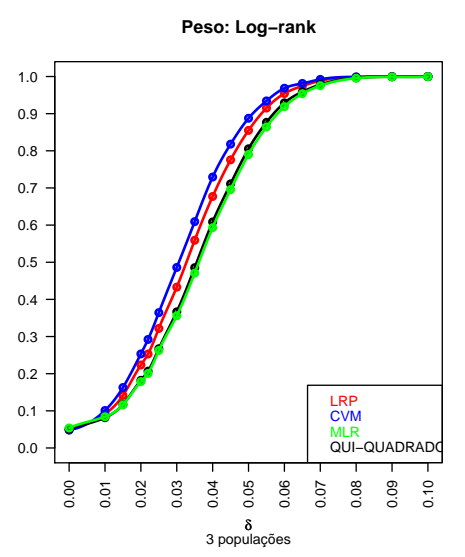

Peso: Fleming e Harrington, Gamma $=0.5$, Rho $=2$

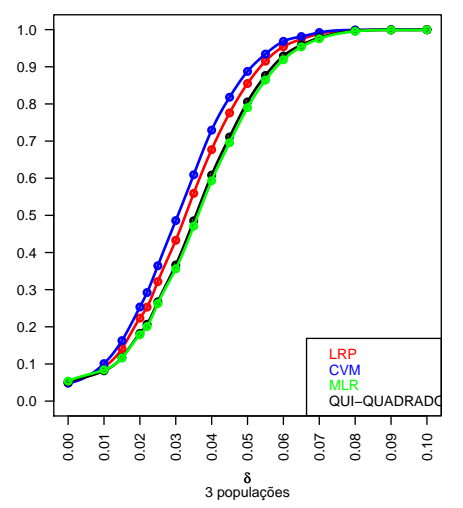

Peso: Gehan

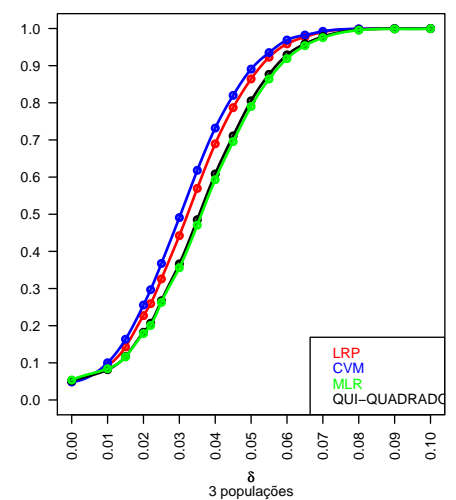

Peso: Peto e Peto

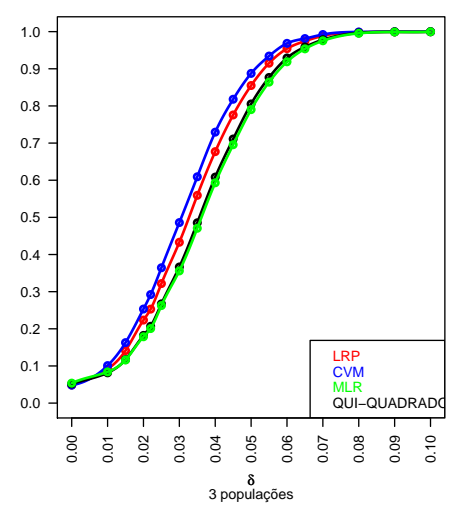

Peso: Tarone e Ware, Gamma $=0.5$

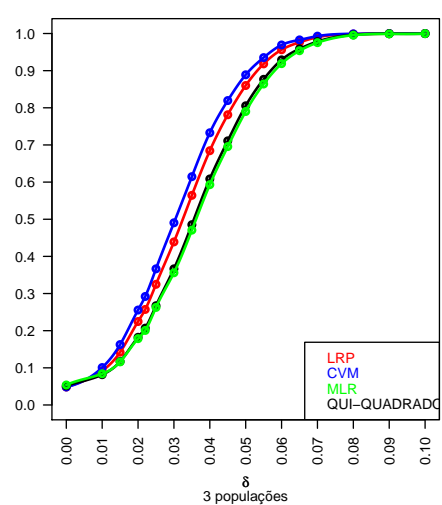

Peso: Peto e Peto Modificado

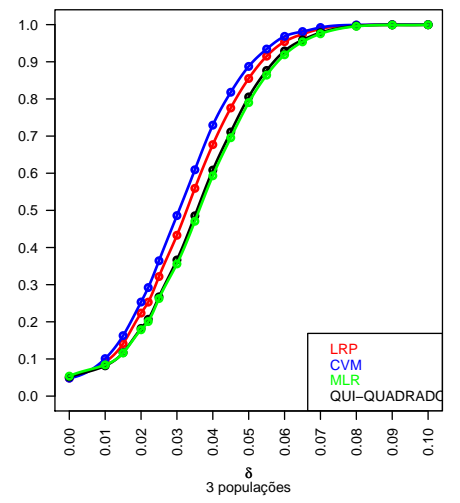

Figura 4.4: Gráfico da Função Poder do teste para 3 populações, tamanho de amostra 500 e sem censura. 


\begin{tabular}{|c|c|c|c|c|c|c|c|c|c|c|c|c|c|c|c|}
\hline \multirow[b]{2}{*}{$\delta$} & \multirow[b]{2}{*}{ Peso } & \multicolumn{4}{|c|}{ Estatística } & \multicolumn{5}{|c|}{ Estatística } & \multicolumn{5}{|c|}{ Estatística } \\
\hline & & LRP & CVM & MLR & QQ & Peso & LRP & CVM & MLR & QQ & Peso & LRP & CVM & MLR & QQ \\
\hline 0 & & 0,0539 & 0,0522 & 0,0535 & 0,0512 & & 0,0534 & 0,0529 & 0,0535 & 0,0501 & & 0,0535 & 0,0524 & 0,0535 & 0,0501 \\
\hline 0,01 & & 0,0789 & 0,0851 & 0,0728 & 0,0712 & & 0,0808 & 0,0852 & 0,0728 & 0,0721 & & 0,0801 & 0,0858 & 0,0728 & 0,0695 \\
\hline 0,015 & & 0,1232 & 0,1447 & 0,1082 & 0,1051 & & 0,1254 & 0,1453 & 0,1082 & 0,1053 & & 0,1239 & 0,1456 & 0,1082 & 0,1053 \\
\hline 0,02 & & 0,1871 & 0,2267 & 0,1553 & 0,1582 & & 0,1907 & 0,2315 & 0,1553 & 0,1582 & & 0,1891 & 0,2298 & 0,1553 & 0,1583 \\
\hline 0,025 & & 0,2832 & 0,3551 & 0,2251 & 0,2383 & & 0,2906 & 0,3578 & 0,2251 & 0,2382 & & 0,2874 & 0,3566 & 0,2251 & 0,2376 \\
\hline 0,03 & & 0,3864 & 0,4686 & 0,3087 & 0,3321 & & 0,3962 & 0,4756 & 0,3087 & 0,3321 & & 0,3915 & 0,4728 & 0,3087 & 0,3316 \\
\hline 0,035 & & 0,5041 & 0,6024 & 0,4088 & 0,4431 & & 0,5151 & 0,6087 & 0,4088 & 0,4432 & & 0,5103 & 0,6068 & 0,4088 & 0,4427 \\
\hline 0,04 & & 0,6421 & 0,7354 & 0,5418 & 0,5812 & & 0,655 & 0,7433 & 0,5418 & 0,5812 & & 0,6481 & 0,7404 & 0,5418 & 0,5805 \\
\hline 0,045 & Log-rank & 0,7455 & 0,8241 & 0,6493 & 0,6911 & Gehan & 0,7535 & 0,8286 & 0,6493 & 0,6912 & Tarone e & 0,7521 & 0,8269 & 0,6493 & 0,6912 \\
\hline 0,05 & & 0,8341 & 0,9008 & 0,7534 & 0,7921 & & 0,8448 & 0,9038 & 0,7534 & 0,7922 & Ware & 0,8397 & 0,9028 & 0,7534 & 0,7918 \\
\hline 0,055 & & 0,9005 & 0,9422 & 0,8314 & 0,8652 & & 0,9074 & 0,9442 & 0,8314 & 0,8652 & $\gamma=0,5$ & 0,9042 & 0,944 & 0,8314 & 0,8648 \\
\hline 0,06 & & 0,9477 & 0,9756 & 0,9017 & 0,9272 & & 0,9543 & 0,9766 & 0,9017 & 0,9273 & & 0,9513 & 0,9767 & 0,9017 & 0,9266 \\
\hline 0,065 & & 0,9752 & 0,9889 & 0,9482 & 0,9622 & & 0,9791 & 0,9885 & 0,9482 & 0,9623 & & 0,9772 & 0,9889 & 0,9482 & 0,9621 \\
\hline 0,07 & & 0,9878 & 0,9954 & 0,9714 & 0,9821 & & 0,9898 & 0,9957 & 0,9714 & 0,9832 & & 0,9886 & 0,9956 & 0,9714 & 0,9803 \\
\hline 0,08 & & 0,9972 & 0,9992 & 0,9945 & 0,9961 & & 0,9973 & 0,9992 & 0,9945 & 0,9962 & & 0,9973 & 0,9993 & 0,9945 & 0,9963 \\
\hline 0,09 & & 0,9999 & 1 & 0,9992 & 1 & & 1 & 1 & 0,9992 & 1 & & 1 & 1 & 0,9992 & 0,9998 \\
\hline 0,1 & & 0,9999 & 1 & 0,9999 & 1 & & 1 & 1 & 0,9999 & 1 & & 1 & 1 & 0,9999 & 0,9999 \\
\hline 0 & & 0,0539 & 0,0522 & 0,0535 & 0,0512 & & 0,0539 & 0,0522 & 0,0535 & 0,0532 & & 0,0539 & 0,0522 & 0,0535 & 0,0501 \\
\hline 0,01 & & 0,0789 & 0,0851 & 0,0728 & 0,0712 & & 0,0789 & 0,0851 & 0,0728 & 0,0732 & & 0,0789 & 0,0851 & 0,0728 & 0,0695 \\
\hline 0,015 & & 0,1231 & 0,1447 & 0,1082 & 0,1052 & & 0,1231 & 0,1447 & 0,1082 & 0,1053 & & 0,123 & 0,1447 & 0,1082 & 0,1053 \\
\hline 0,02 & & 0,1871 & 0,2267 & 0,1553 & 0,1582 & & 0,1871 & 0,2267 & 0,1553 & 0,1582 & & 0,1871 & 0,2267 & 0,1553 & 0,1583 \\
\hline 0,025 & & 0,2832 & 0,3551 & 0,2251 & 0,2382 & & 0,2832 & 0,3553 & 0,2252 & 0,2382 & & 0,2832 & 0,3551 & 0,2253 & 0,2376 \\
\hline 0,03 & & 0,3864 & 0,4686 & 0,3087 & 0,3321 & & 0,3864 & 0,4686 & 0,3087 & 0,3323 & & 0,3864 & 0,4686 & 0,3087 & 0,3316 \\
\hline 0,035 & & 0,5041 & 0,6024 & 0,4088 & 0,4431 & & 0,5041 & 0,6024 & 0,4088 & 0,4432 & & 0,5041 & 0,6024 & 0,4088 & 0,4427 \\
\hline 0,04 & & 0,6421 & 0,7354 & 0,5418 & 0,5812 & & 0,642 & 0,7354 & 0,5418 & 0,5812 & & 0,642 & 0,7354 & 0,5418 & 0,5805 \\
\hline 0,045 & Fleming e & 0,7455 & 0,8241 & 0,6493 & 0,6911 & Peto e & 0,7455 & 0,8241 & 0,6493 & 0,6912 & Peto e & 0,7455 & 0,8241 & 0,6493 & 0,6912 \\
\hline 0,05 & Harrington & 0,8341 & 0,9008 & 0,7534 & 0,7922 & Peto & 0,8341 & 0,9008 & 0,7534 & 0,7922 & Peto & 0,8341 & 0,9008 & 0,7534 & 0,7918 \\
\hline 0,055 & $\gamma=0,5 \mathrm{e}$ & 0,9005 & 0,9422 & 0,8314 & 0,865 & & 0,9005 & 0,9422 & 0,8314 & 0,8652 & Modificado & 0,9005 & 0,9422 & 0,8314 & 0,8648 \\
\hline 0,06 & $\rho=2$ & 0,9477 & 0,9756 & 0,9017 & 0,9271 & & 0,9477 & 0,9756 & 0,9017 & 0,9272 & & 0,9477 & 0,9756 & 0,9017 & 0,9266 \\
\hline 0,065 & & 0,9752 & 0,9889 & 0,9482 & 0,9622 & & 0,9752 & 0,9889 & 0,9482 & 0,9623 & & 0,9752 & 0,9889 & 0,9482 & 0,9622 \\
\hline 0,07 & & 0,9878 & 0,9954 & 0,9714 & 0,9812 & & 0,9878 & 0,9954 & 0,9714 & 0,9832 & & 0,9878 & 0,9954 & 0,9714 & 0,9803 \\
\hline 0,08 & & 0,9972 & 0,9992 & 0,9945 & 0,9962 & & 0,9972 & 0,9992 & 0,9945 & 0,9963 & & 0,9972 & 0,9992 & 0,9945 & 0,9963 \\
\hline 0,09 & & 0,9999 & 1 & 0,9992 & 1 & & 0,9999 & 1 & 0,9992 & 1 & & 0,9999 & 1 & 0,9992 & 0,9998 \\
\hline 0,1 & & 0,9999 & 1 & 0,9999 & 1 & & 0,9999 & 1 & 0,9999 & 1 & & 0,9999 & 1 & 0,9999 & 0,9999 \\
\hline
\end{tabular}

Tabela 4.4: Função Poder do teste para 5 populações, tamanho de amostra 500 e sem censura.

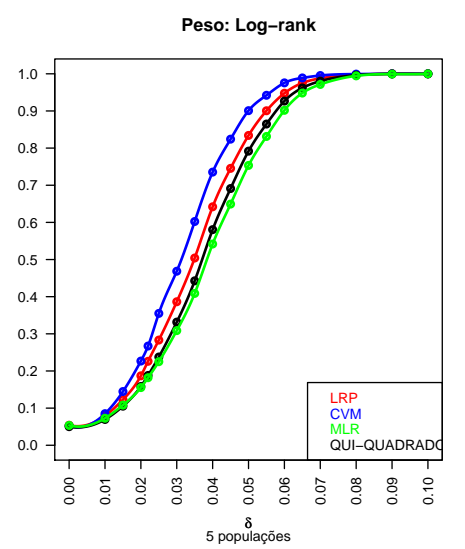

Peso: Fleming e Harrington, Gamma $=0.5, \mathrm{Rho}=2$

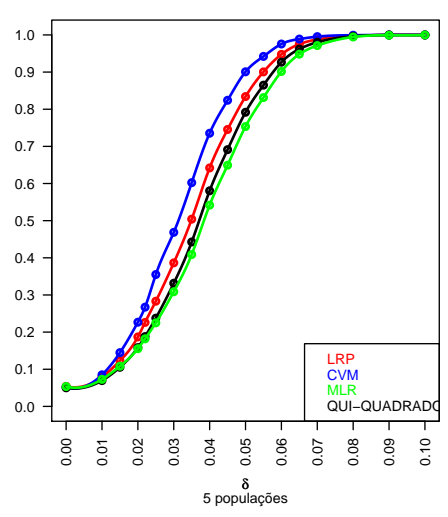

Peso: Gehan

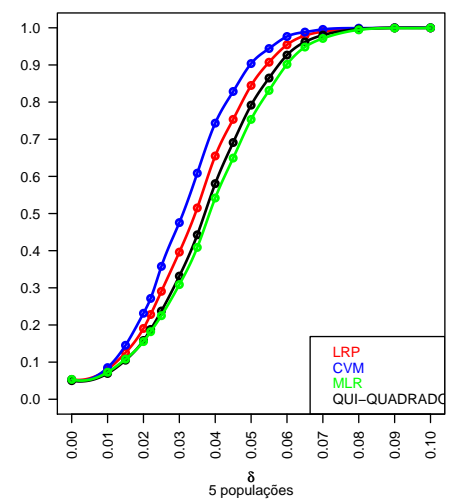

Peso: Peto e Peto

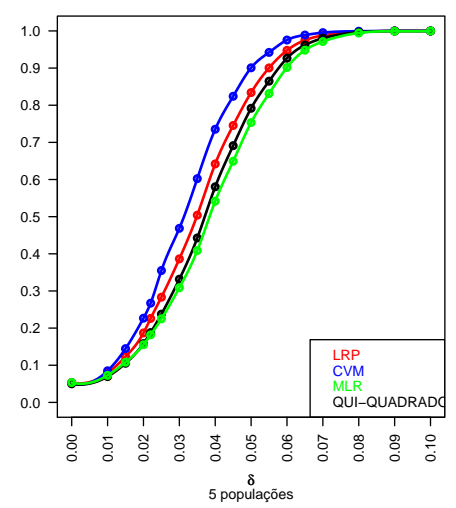

Peso: Tarone e Ware, Gamma $=0.5$

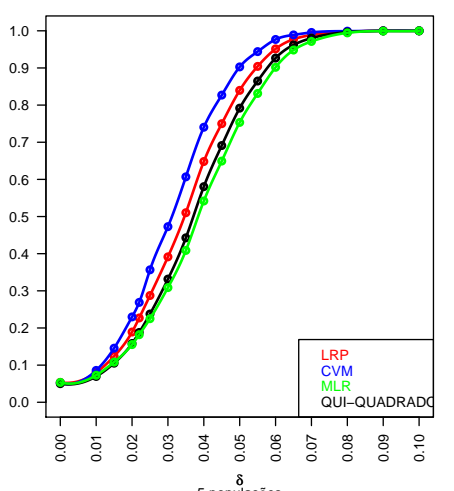

Peso: Peto e Peto Modificado

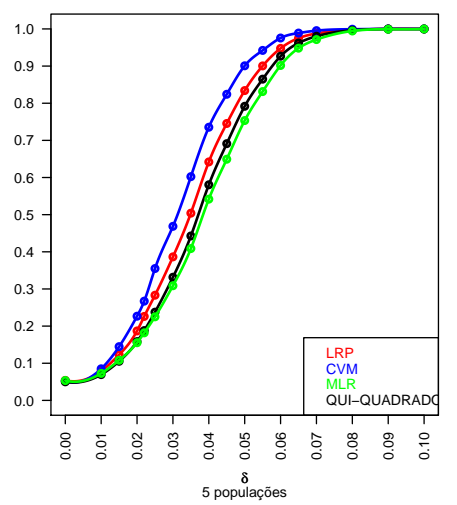

Figura 4.5: Gráfico da Função Poder do teste para 5 populações, tamanho de amostra 500 e sem censura. 
Resumindo os resultados obtidos para 2, 3 e 5 populações, observamos que as estatísticas de Cramérvon Mises e Log-rank Ponderado tem um comportamento similar e um pouco melhor que as estatística de MLR e Qui-quadrado independente do número de populações.

Na sequência, avaliamos o poder do teste quando a hipótese alternativa é fixada e variamos o tamanho de amostra. Neste experimento, adotamos a mesma metodologia utilizada no experimento anterior, porém, com a seguinte mudança. Primeiramente, fixamos o valor de $\delta$ em 0,05 , e variamos o tamanho da amostra. Assim, o objetivo é avaliar a função poder do teste quando aumentamos os tamanhos de amostra para todas as estatísticas. No experimento, os tamanhos de amostra escolhidos para a simulação são 50, 80, 100, 135, $170,180,190,200,250,300,350,400,450,500,550$ e 600.

1. Fixamos $\delta=0,05$ e o nível de significância $\alpha=0,05$;

2. Escolhemos um tamanho da amostra $T A$ e definimos $J(2,3$ e 5$)$ populações com distribuição Multinomial:

- $X^{(1)}, \ldots, X^{(J)} \sim M\left(T A, p_{1}, p_{2}, p_{3}\right)$

- A função de intensidade para a população de controle $h^{n_{1}}=\left(\begin{array}{lll}0,1+\delta & 0,2+\delta & 1\end{array}\right)$;

- A função de intensidade para as populações de tratamento $h^{n_{2}}=\cdots=h^{n_{J}}=\left(\begin{array}{lll}0,1 & 0,2 & 1\end{array}\right)$;

- À partir das funções de intensidade, calculamos as probabilidades $p_{1}, p_{2}$ e $p_{3}$ usando a equação (2.5), ou seja, $\pi_{i}=P[W=i]=h(i) \prod_{\ell=1}^{i-1}[1-h(\ell)]$ dado que a hipótese nula é falsa;

- Escolhemos uma das funções de ponderação descrita na tabela (3.1);

3. Realizamos o teste de hipótese usando valor crítico ao nível de significância $\alpha$;

4. Para estimarmos a função poder tomamos

$$
y_{m}= \begin{cases}1, & \text { se } H_{0} \text { foi rejeitada ao nível de significância } \alpha \\ 0, & \text { caso contrário }\end{cases}
$$

5. Repetimos as etapas 2 a $4 m=10000$ vezes;

6. Estimamos o poder do teste na seguinte forma:

$$
\text { Poder }=\frac{1}{m} \sum_{k=1}^{m} y_{k}
$$




\begin{tabular}{|c|c|c|c|c|c|c|c|c|c|c|c|c|c|c|c|}
\hline \multirow[b]{2}{*}{ TA } & \multirow[b]{2}{*}{ Peso } & \multicolumn{4}{|c|}{ Estatística } & \multicolumn{5}{|c|}{ Estatística } & \multicolumn{5}{|c|}{ Estatística } \\
\hline & & LRP & CVM & MLR & QQ & Peso & LRP & CVM & MLR & QQ & Peso & LRP & CVM & MLR & QQ \\
\hline 50 & & 0,1831 & 0,1845 & 0,1516 & 0,1412 & & 0,186 & 0,185 & 0,1516 & 0,1411 & & 0,185 & 0,1844 & 0,1516 & 0,1407 \\
\hline 80 & & 0,222 & 0,2304 & 0,1843 & 0,1742 & & 0,228 & 0,2311 & 0,1843 & 0,1742 & & 0,2246 & 0,2312 & 0,1843 & 0,1737 \\
\hline 100 & & 0,2599 & 0,2657 & 0,2137 & 0,2063 & & 0,2648 & 0,2654 & 0,2137 & 0,2061 & & 0,2619 & 0,2656 & 0,2137 & 0,2061 \\
\hline 120 & & 0,3042 & 0,3145 & 0,243 & 0,237 & & 0,3098 & 0,3181 & 0,243 & 0,237 & & 0,3078 & 0,3157 & 0,243 & 0,2367 \\
\hline 135 & & 0,3315 & 0,3445 & 0,2731 & 0,2653 & & 0,3372 & 0,3456 & 0,2731 & 0,2652 & & 0,3336 & 0,3461 & 0,2731 & 0,2653 \\
\hline 170 & & 0,3945 & 0,4094 & 0,3175 & 0,3132 & & 0,4027 & 0,4087 & 0,3175 & 0,3132 & & 0,398 & 0,4106 & 0,3175 & 0,3132 \\
\hline 180 & & 0,4111 & 0,4243 & 0,3368 & 0,3311 & & 0,4199 & 0,4249 & 0,3368 & 0,3312 & & 0,4147 & 0,4249 & 0,3368 & 0,3309 \\
\hline 190 & Log-rank & 0,4349 & 0,4466 & 0,3624 & 0,3582 & Gehan & 0,4445 & 0,4455 & 0,3624 & 0,3582 & Tarone e & 0,4395 & 0,4458 & 0,3624 & 0,3577 \\
\hline 200 & & 0,4487 & 0,4636 & 0,3711 & 0,3673 & & 0,4567 & 0,4611 & 0,3711 & 0,3671 & Ware & 0,454 & 0,4616 & 0,3711 & 0,367 \\
\hline 220 & & 0,4974 & 0,5135 & 0,414 & 0,41 & & 0,5075 & 0,5155 & 0,414 & 0,41 & & 0,5026 & 0,516 & 0,414 & 0,4103 \\
\hline 250 & & 0,5407 & 0,5642 & 0,4674 & 0,4644 & & 0,5525 & 0,5682 & 0,4674 & 0,4641 & & 0,5468 & 0,5681 & 0,4674 & 0,4636 \\
\hline 300 & & 0,6082 & 0,6311 & 0,5304 & 0,5274 & & 0,6212 & 0,6322 & 0,5304 & 0,5272 & & 0,6154 & 0,6338 & 0,5304 & 0,5269 \\
\hline 350 & & 0,6826 & 0,7042 & 0,6049 & 0,6024 & & 0,6966 & 0,7063 & 0,6049 & 0,6022 & & 0,6917 & 0,7072 & 0,6049 & 0,6018 \\
\hline 400 & & 0,7442 & 0,7647 & 0,6685 & 0,6674 & & 0,7562 & 0,7628 & 0,6685 & 0,6671 & & 0,7511 & 0,7658 & 0,6685 & 0,6671 \\
\hline 450 & & 0,7923 & 0,8126 & 0,7224 & 0,7223 & & 0,8045 & 0,8131 & 0,7224 & 0,7212 & & 0,7991 & 0,8147 & 0,7224 & 0,7223 \\
\hline 500 & & 0,8261 & 0,8425 & 0,7665 & 0,7653 & & 0,8347 & 0,8428 & 0,7665 & 0,7652 & & 0,8313 & 0,8441 & 0,7665 & 0,7649 \\
\hline 600 & & 0,8883 & 0,9059 & 0,8407 & 0,8444 & & 0,8982 & 0,9076 & 0,8407 & 0,8412 & & 0,8932 & 0,9082 & 0,8407 & 0,8397 \\
\hline 700 & & 0,9318 & 0,9415 & 0,8976 & 0,897 & & 0,9369 & 0,9413 & 0,8976 & 0,897 & & 0,9341 & 0,9422 & 0,8976 & 0,8972 \\
\hline 50 & & 0,1831 & 0,1845 & 0,1516 & 0,1412 & & 0,1831 & 0,1845 & 0,1516 & 0,1413 & & 0,1831 & 0,1845 & 0,1516 & 0,1407 \\
\hline 80 & & 0,2221 & 0,2304 & 0,1843 & 0,1742 & & 0,2222 & 0,2304 & 0,1843 & 0,1744 & & 0,2223 & 0,2304 & 0,1843 & 0,1737 \\
\hline 100 & & 0,2599 & 0,2657 & 0,2137 & 0,2064 & & 0,2599 & 0,2657 & 0,2137 & 0,2064 & & 0,2599 & 0,2657 & 0,2137 & 0,2061 \\
\hline 120 & & 0,3042 & 0,3145 & 0,243 & 0,237 & & 0,3042 & 0,3145 & 0,243 & 0,237 & & 0,3042 & 0,3145 & 0,243 & 0,2367 \\
\hline 135 & & 0,3315 & 0,3445 & 0,2731 & 0,2654 & & 0,3315 & 0,3445 & 0,2731 & 0,265 & & 0,3315 & 0,3445 & 0,2731 & 0,2653 \\
\hline 170 & Fleming e & 0,3945 & 0,4094 & 0,3175 & 0,3134 & Peto e & 0,3945 & 0,4094 & 0,3175 & 0,3132 & Peto e & 0,3945 & 0,4094 & 0,3175 & 0,3132 \\
\hline 180 & Harrington & 0,4111 & 0,4243 & 0,3368 & 0,3312 & Peto & 0,4111 & 0,4243 & 0,3368 & 0,3314 & Peto & 0,4111 & 0,4243 & 0,3368 & 0,3309 \\
\hline 190 & $\gamma=0,5 \mathrm{e}$ & 0,4349 & 0,4466 & 0,3624 & 0,3584 & & 0,4349 & 0,4466 & 0,3624 & 0,3582 & Modificado & 0,4349 & 0,4466 & 0,3624 & 0,3577 \\
\hline 200 & $\rho=2$ & 0,4487 & 0,4636 & 0,3711 & 0,3672 & & 0,4487 & 0,4636 & 0,3711 & 0,3672 & & 0,4487 & 0,4636 & 0,3711 & 0,3674 \\
\hline 220 & & 0,4974 & 0,5135 & 0,414 & 0,41 & & 0,4974 & 0,5135 & 0,414 & 0,41 & & 0,4974 & 0,5135 & 0,414 & 0,4103 \\
\hline 250 & & 0,5407 & 0,5642 & 0,4674 & 0,4644 & & 0,5407 & 0,5642 & 0,4674 & 0,4644 & & 0,5407 & 0,5642 & 0,4674 & 0,4636 \\
\hline 300 & & 0,6082 & 0,6311 & 0,5304 & 0,5274 & & 0,6082 & 0,6311 & 0,5304 & 0,5272 & & 0,6082 & 0,6311 & 0,5304 & 0,5269 \\
\hline 350 & & 0,6826 & 0,7044 & 0,6049 & 0,6023 & & 0,6826 & 0,704 & 0,6049 & 0,6022 & & 0,6826 & 0,7042 & 0,6049 & 0,6018 \\
\hline 400 & & 0,7442 & 0,7647 & 0,6685 & 0,6674 & & 0,7442 & 0,7647 & 0,6685 & 0,6674 & & 0,7442 & 0,7647 & 0,6685 & 0,6671 \\
\hline 450 & & 0,7923 & 0,8126 & 0,7224 & 0,7223 & & 0,7923 & 0,8126 & 0,7224 & 0,7234 & & 0,7923 & 0,8126 & 0,7224 & 0,7212 \\
\hline 500 & & 0,8261 & 0,8425 & 0,7665 & 0,7653 & & 0,8261 & 0,8425 & 0,7665 & 0,7653 & & 0,8261 & 0,8425 & 0,7665 & 0,7649 \\
\hline 600 & & 0,8883 & 0,9059 & 0,8407 & 0,8432 & & 0,8883 & 0,9059 & 0,8407 & 0,8423 & & 0,8883 & 0,9059 & 0,8407 & 0,8397 \\
\hline 700 & & 0,9318 & 0,9415 & 0,8976 & 0,897 & & 0,9318 & 0,9415 & 0,8976 & 0,897 & & 0,9318 & 0,9415 & 0,8976 & 0,8972 \\
\hline
\end{tabular}

Tabela 4.5: Função Poder do teste para 2 populações, $\delta$ de 0,05 e sem censura.

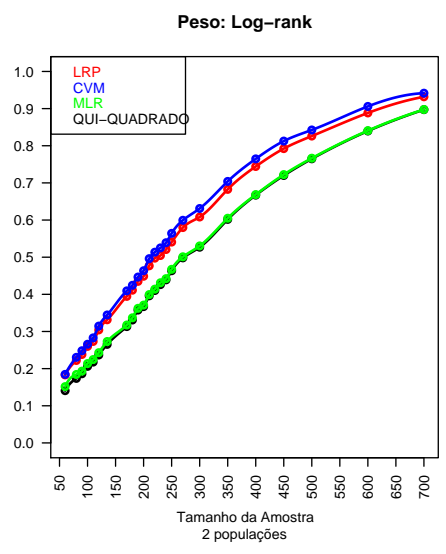

Peso: Fleming e Harrington, Gamma=0.5,Rho $=2$

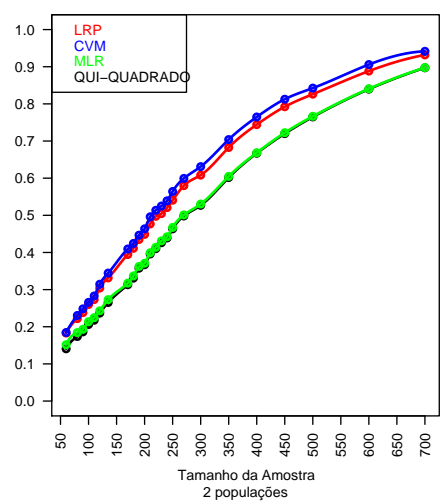

Peso: Gehan

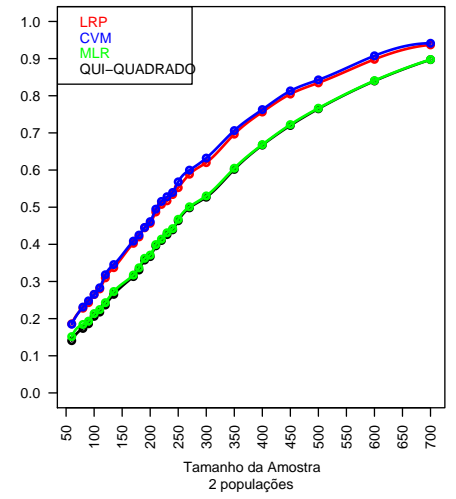

Peso: Peto e Peto

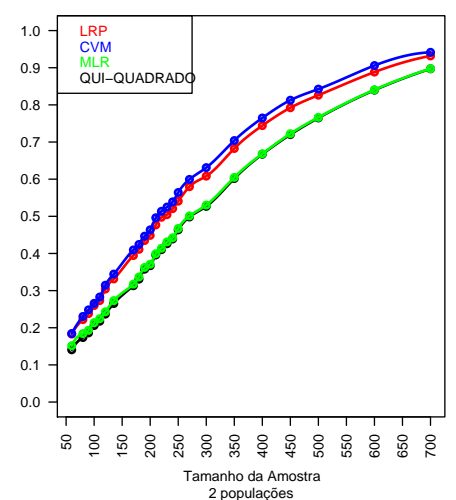

Peso: Tarone Ware, Gamma=0.5

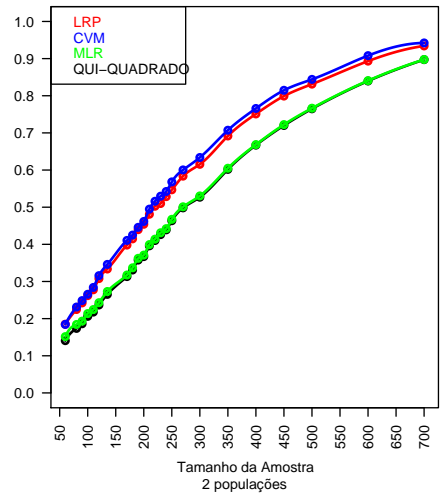

Peso: Peto e Peto Modificado

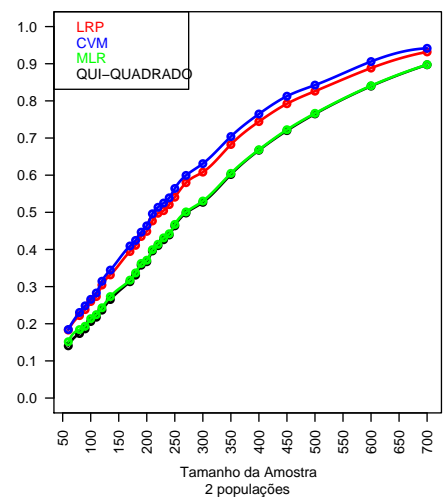

Figura 4.6: Gráfico da Função Poder do teste para 2 populações, $\delta$ de 0,05 e sem censura. 


\begin{tabular}{|c|c|c|c|c|c|c|c|c|c|c|c|c|c|c|c|}
\hline \multirow[b]{2}{*}{ TA } & \multirow[b]{2}{*}{ Peso } & \multicolumn{4}{|c|}{ Estatística } & \multicolumn{5}{|c|}{ Estatística } & \multicolumn{5}{|c|}{ Estatística } \\
\hline & & LRP & CVM & MLR & QQ & Peso & LRP & CVM & MLR & QQ & Peso & LRP & CVM & MLR & QQ \\
\hline 50 & & 0,1631 & 0,1794 & 0,1505 & 0,1311 & & 0,1662 & 0,1796 & 0,1505 & 0,1314 & & 0,1647 & 0,179 & 0,1505 & 0,1311 \\
\hline 80 & & 0,2074 & 0,238 & 0,1776 & 0,1652 & & 0,2144 & 0,2378 & 0,1776 & 0,1653 & & 0,2113 & 0,2377 & 0,1776 & 0,1653 \\
\hline 100 & & 0,2496 & 0,2863 & 0,2009 & 0,2234 & & 0,2537 & 0,2877 & 0,2009 & 0,2244 & & 0,2515 & 0,2876 & 0,2009 & 0,1998 \\
\hline 120 & & 0,2867 & 0,3272 & 0,227 & 0,229 & & 0,2926 & 0,3276 & 0,227 & 0,229 & & 0,2904 & 0,3273 & 0,227 & 0,2291 \\
\hline 135 & & 0,3268 & 0,3705 & 0,2643 & 0,2681 & & 0,3322 & 0,3699 & 0,2643 & 0,2682 & & 0,3305 & 0,3705 & 0,2643 & 0,2681 \\
\hline 170 & & 0,3861 & 0,4412 & 0,3197 & 0,3261 & & 0,3955 & 0,4435 & 0,3197 & 0,3264 & & 0,3911 & 0,4442 & 0,3197 & 0,3262 \\
\hline 180 & & 0,4108 & 0,4642 & 0,3358 & 0,3523 & & 0,4188 & 0,4677 & 0,3358 & 0,3543 & & 0,4132 & 0,4655 & 0,3358 & 0,3498 \\
\hline 190 & Log-rank & 0,4314 & 0,4876 & 0,3549 & 0,3713 & Gehan & 0,4389 & 0,4887 & 0,3549 & 0,3712 & Tarone e & 0,4352 & 0,49 & 0,3549 & 0,3707 \\
\hline 200 & & 0,4477 & 0,5094 & 0,3733 & 0,3861 & & 0,4577 & 0,5091 & 0,3733 & 0,3864 & Ware $\gamma=0,5$ & 0,4535 & 0,5117 & 0,3733 & 0,3858 \\
\hline 220 & & 0,4951 & 0,5483 & 0,4073 & 0,423 & & 0,5068 & 0,5503 & 0,4073 & 0,423 & & 0,501 & 0,5504 & 0,4073 & 0,4227 \\
\hline 250 & & 0,5461 & 0,5992 & 0,4541 & 0,4722 & & 0,5584 & 0,6043 & 0,4541 & 0,4724 & & 0,5521 & 0,6021 & 0,4541 & 0,4719 \\
\hline 300 & & 0,6298 & 0,6869 & 0,5387 & 0,5573 & & 0,6411 & 0,6893 & 0,5387 & 0,5573 & & 0,6363 & 0,6891 & 0,5387 & 0,5572 \\
\hline 350 & & 0,7066 & 0,7488 & 0,6162 & 0,6371 & & 0,7192 & 0,7534 & 0,6162 & 0,6374 & & 0,7139 & 0,7543 & 0,6162 & 0,6365 \\
\hline 400 & & 0,7607 & 0,8108 & 0,6759 & 0,6941 & & 0,7735 & 0,8136 & 0,6759 & 0,6942 & & 0,7691 & 0,8127 & 0,6759 & 0,6942 \\
\hline 450 & & 0,8165 & 0,8518 & 0,7414 & 0,7582 & & 0,8261 & 0,8562 & 0,7414 & 0,7583 & & 0,8216 & 0,8537 & 0,7414 & 0,7579 \\
\hline 500 & & 0,8542 & 0,885 & 0,7893 & 0,8042 & & 0,8642 & 0,8884 & 0,7893 & 0,8042 & & 0,8594 & 0,8874 & 0,7893 & 0,8044 \\
\hline 600 & & 0,9099 & 0,9337 & 0,8609 & 0,8732 & & 0,9169 & 0,9345 & 0,8609 & 0,8733 & & 0,9131 & 0,9346 & 0,8609 & 0,8733 \\
\hline 700 & & 0,9507 & 0,963 & 0,915 & 0,923 & & 0,9546 & 0,965 & 0,915 & 0,923 & & 0,9527 & 0,9643 & 0,915 & 0,9227 \\
\hline 50 & & 0,1631 & 0,1794 & 0,1505 & 0,1312 & & 0,1631 & 0,1794 & 0,1505 & 0,1312 & & 0,1631 & 0,1794 & 0,1505 & 0,1311 \\
\hline 80 & & 0,2074 & 0,2382 & 0,1776 & 0,1652 & & 0,2074 & 0,2381 & 0,1776 & 0,1653 & & 0,2074 & 0,238 & 0,1776 & 0,1653 \\
\hline 100 & & 0,2496 & 0,2863 & 0,2009 & 0,2234 & & 0,2496 & 0,2863 & 0,2009 & 0,2231 & & 0,2496 & 0,2863 & 0,2009 & 0,1998 \\
\hline 120 & & 0,2867 & 0,3272 & 0,227 & 0,229 & & 0,2867 & 0,3272 & 0,227 & 0,229 & & 0,2867 & 0,3272 & 0,227 & 0,2291 \\
\hline 135 & & 0,3268 & 0,3705 & 0,2643 & 0,2681 & & 0,3268 & 0,3705 & 0,2643 & 0,2683 & & 0,3268 & 0,3705 & 0,2643 & 0,2681 \\
\hline 170 & & 0,3861 & 0,4412 & 0,3197 & 0,3262 & & 0,3861 & 0,4412 & 0,3197 & 0,3263 & & 0,3861 & 0,4412 & 0,3197 & 0,3262 \\
\hline 180 & & 0,4108 & 0,4642 & 0,3358 & 0,3523 & & 0,4108 & 0,4642 & 0,3358 & 0,3543 & & 0,4108 & 0,4642 & 0,3358 & 0,3498 \\
\hline 190 & Fleming e & 0,4314 & 0,4876 & 0,3549 & 0,3712 & Peto e & 0,4314 & 0,4876 & 0,3549 & 0,3714 & Peto e & 0,4314 & 0,4876 & 0,3549 & 0,3707 \\
\hline 200 & Harrington $\gamma=0,5$ & 0,4477 & 0,5094 & 0,3733 & 0,3862 & Peto & 0,4477 & 0,5094 & 0,3733 & 0,3863 & Peto & 0,4477 & 0,5094 & 0,3733 & 0,3858 \\
\hline 220 & $\rho=2$ & 0,4951 & 0,5483 & 0,4073 & 0,423 & & 0,4951 & 0,5483 & 0,4073 & 0,423 & & 0,4951 & 0,5483 & 0,4073 & 0,4227 \\
\hline 250 & $\rho=2$ & 0,5462 & 0,5992 & 0,4541 & 0,4722 & & 0,5461 & 0,5992 & 0,4541 & 0,4723 & & 0,5462 & 0,5992 & 0,4541 & 0,4719 \\
\hline 300 & & 0,6298 & 0,6869 & 0,5387 & 0,5571 & & 0,6298 & 0,6869 & 0,5387 & 0,5572 & & 0,6298 & 0,6869 & 0,5387 & 0,5572 \\
\hline 350 & & 0,7066 & 0,7488 & 0,6162 & 0,6372 & & 0,7066 & 0,7488 & 0,6162 & 0,6372 & & 0,7066 & 0,7488 & 0,6162 & 0,6365 \\
\hline 400 & & 0,7607 & 0,8108 & 0,6759 & 0,6942 & & 0,7607 & 0,8108 & 0,6759 & 0,6943 & & 0,7607 & 0,8108 & 0,6759 & 0,6942 \\
\hline 450 & & 0,8165 & 0,8518 & 0,7414 & 0,7581 & & 0,8165 & 0,8518 & 0,7414 & 0,7582 & & 0,8165 & 0,8518 & 0,7414 & 0,7579 \\
\hline 500 & & 0,8542 & 0,885 & 0,7893 & 0,8042 & & 0,8542 & 0,8851 & 0,7893 & 0,8043 & & 0,8542 & 0,8852 & 0,7893 & 0,8044 \\
\hline 600 & & 0,9099 & 0,9337 & 0,8609 & 0,8733 & & 0,9099 & 0,9337 & 0,8609 & 0,8732 & & 0,9099 & 0,9337 & 0,8609 & 0,8733 \\
\hline 700 & & 0,9507 & 0,963 & 0,915 & 0,923 & & 0,9507 & 0,963 & 0,915 & 0,923 & & 0,9507 & 0,963 & 0,915 & 0,9227 \\
\hline
\end{tabular}

Tabela 4.6: Função Poder do teste para 3 populações, $\delta$ de 0,05 e sem censura.

Peso: Log-rank

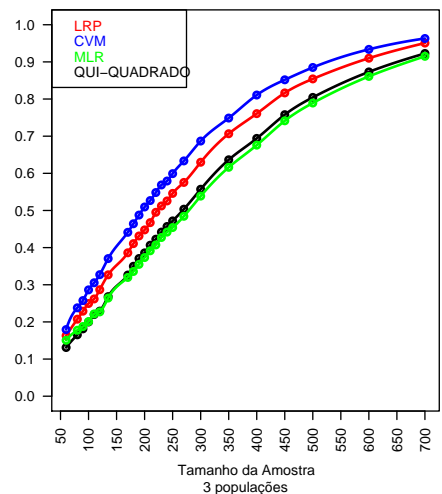

Peso: Fleming e Harrington, Gamma $=0.5, \mathrm{Rho}=2$

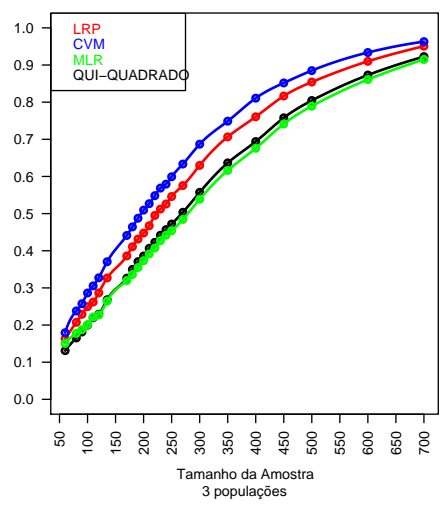

Peso: Gehan

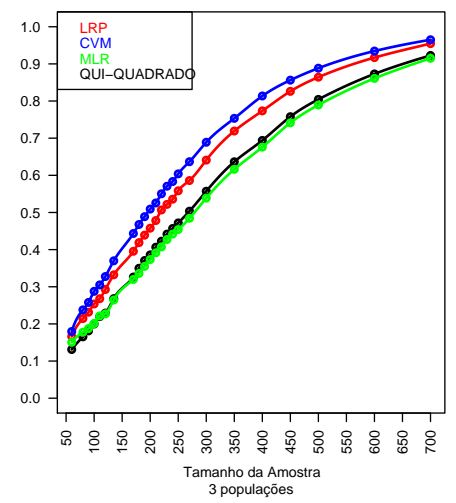

Peso: Peto e Peto

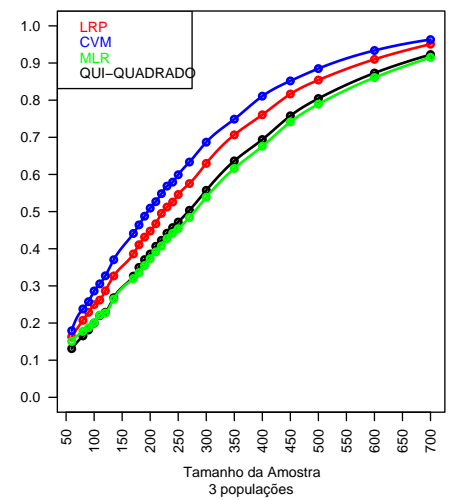

Peso: Tarone e Ware, Gamma $=0.5$

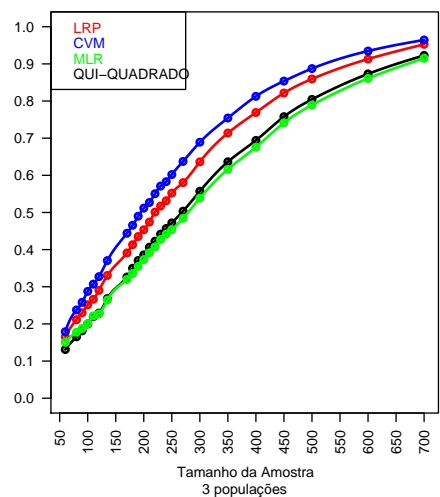

Peso: Peto e Peto Modificado

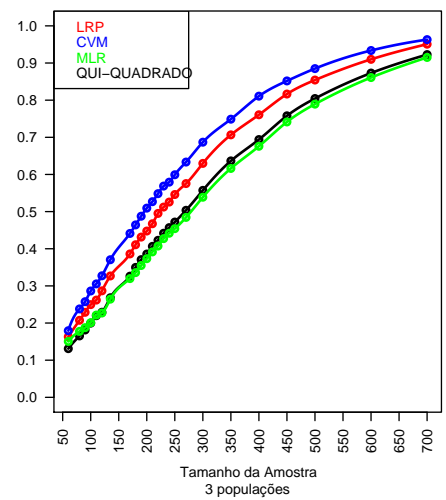

Figura 4.7: Gráfico da Função Poder do teste para 3 populações, $\delta$ de 0,05 e sem censura. 


\begin{tabular}{|c|c|c|c|c|c|c|c|c|c|c|c|c|c|c|c|}
\hline \multirow[b]{2}{*}{ TA } & \multirow[b]{2}{*}{ Peso } & \multicolumn{4}{|c|}{ Estatística } & \multicolumn{5}{|c|}{ Estatística } & \multicolumn{5}{|c|}{ Estatística } \\
\hline & & LRP & CVM & MLR & QQ & Peso & LRP & CVM & MLR & QQ & Peso & LRP & CVM & MLR & QQ \\
\hline 50 & & 0,1562 & 0,1654 & 0,1643 & 0,1212 & & 0,1596 & 0,1649 & 0,1643 & 0,1212 & & 0,1579 & 0,1661 & 0,164 & 0,1207 \\
\hline 80 & & 0,181 & 0,2126 & 0,1735 & 0,1482 & & 0,1833 & 0,2107 & 0,1735 & 0,1482 & & 0,1816 & 0,2114 & 0,1735 & 0,1482 \\
\hline 100 & & 0,2168 & 0,2625 & 0,1911 & 0,1823 & & 0,2227 & 0,2663 & 0,1911 & 0,1832 & & 0,2201 & 0,2659 & 0,1911 & 0,1795 \\
\hline 120 & & 0,2577 & 0,3177 & 0,2146 & 0,209 & & 0,2614 & 0,3205 & 0,2146 & 0,209 & & 0,2603 & 0,3204 & 0,2146 & 0,209 \\
\hline 135 & & 0,2847 & 0,3635 & 0,2419 & 0,2483 & & 0,2922 & 0,3669 & 0,2419 & 0,2482 & & 0,2886 & 0,367 & 0,2419 & 0,2482 \\
\hline 170 & & 0,3433 & 0,4364 & 0,2824 & 0,3021 & & 0,3521 & 0,4386 & 0,2824 & 0,3022 & & 0,3464 & 0,4383 & 0,2824 & 0,3023 \\
\hline 180 & & 0,3713 & 0,4645 & 0,2997 & 0,3262 & & 0,3786 & 0,4694 & 0,2997 & 0,3264 & & 0,3747 & 0,4675 & 0,2997 & 0,3261 \\
\hline 190 & Log-rank & 0,3893 & 0,4926 & 0,3147 & 0,3421 & Gehan & 0,3963 & 0,4958 & 0,3147 & 0,3422 & Tarone e & 0,3936 & 0,4952 & 0,3147 & 0,3418 \\
\hline 200 & & 0,4061 & 0,5058 & 0,3274 & 0,3573 & & 0,4153 & 0,5114 & 0,3274 & 0,3572 & Ware & 0,4107 & 0,5099 & 0,3274 & 0,3573 \\
\hline 220 & $\gamma=0,5$ & 0,4433 & 0,5414 & 0,3605 & 0,3932 & & 0,4538 & 0,5462 & 0,3605 & 0,3933 & & 0,4485 & 0,5452 & 0,3605 & 0,3933 \\
\hline 250 & & 0,4976 & 0,5992 & 0,4013 & 0,4423 & & 0,5071 & 0,6057 & 0,4013 & 0,4432 & & 0,5023 & 0,6031 & 0,4013 & 0,4404 \\
\hline 300 & & 0,5927 & 0,6933 & 0,4925 & 0,5332 & & 0,6039 & 0,7004 & 0,4925 & 0,5332 & & 0,5984 & 0,6981 & 0,4925 & 0,5333 \\
\hline 350 & & 0,6662 & 0,7602 & 0,5638 & 0,6092 & & 0,6784 & 0,7649 & 0,5638 & 0,6092 & & 0,6737 & 0,7639 & 0,5638 & 0,6087 \\
\hline 400 & & 0,7274 & 0,8183 & 0,6314 & 0,6753 & & 0,7402 & 0,8217 & 0,6314 & 0,6751 & & 0,734 & 0,8211 & 0,6314 & 0,6751 \\
\hline 450 & & 0,7865 & 0,8624 & 0,695 & 0,7371 & & 0,7974 & 0,8674 & 0,6953 & 0,7372 & & 0,7918 & 0,8657 & 0,695 & 0,7366 \\
\hline 500 & & 0,8356 & 0,8994 & 0,7511 & 0,7871 & & 0,8463 & 0,9028 & 0,7511 & 0,7872 & & 0,8422 & 0,9025 & 0,7511 & 0,7869 \\
\hline 600 & & 0,9033 & 0,9466 & 0,8404 & 0,8723 & & 0,9117 & 0,9484 & 0,8404 & 0,8712 & & 0,9082 & 0,9486 & 0,8404 & 0,8701 \\
\hline 700 & & 0,9448 & 0,9719 & 0,9065 & 0,927 & & 0,9509 & 0,9737 & 0,9065 & 0,927 & & 0,9486 & 0,973 & 0,9065 & 0,9265 \\
\hline 50 & & 0,1562 & 0,1654 & 0,1642 & 0,1211 & & 0,1562 & 0,1654 & 0,1641 & 0,1213 & & 0,1562 & 0,1654 & 0,164 & 0,1207 \\
\hline 80 & & 0,1811 & 0,2126 & 0,1735 & 0,1483 & & 0,181 & 0,2126 & 0,1735 & 0,1483 & & 0,181 & 0,2126 & 0,1735 & 0,1482 \\
\hline 100 & & 0,2168 & 0,2625 & 0,1911 & 0,1812 & & 0,2168 & 0,2625 & 0,1911 & 0,1832 & & 0,2168 & 0,2625 & 0,1911 & 0,1795 \\
\hline 120 & & 0,2577 & 0,3177 & 0,2146 & 0,209 & & 0,2577 & 0,3177 & 0,2146 & 0,209 & & 0,2577 & 0,3177 & 0,2146 & 0,209 \\
\hline 135 & & 0,2847 & 0,3635 & 0,2419 & 0,2481 & & 0,2847 & 0,3635 & 0,2419 & 0,2483 & & 0,2847 & 0,3635 & 0,2419 & 0,2482 \\
\hline 180 & & 0,3713 & 0,4645 & 0,2997 & 0,3262 & & 0,3713 & 0,4645 & 0,2997 & 0,3263 & & 0,3713 & 0,4645 & 0,2997 & 0,3263 \\
\hline 190 & Fleming e & 0,3893 & 0,4926 & 0,3147 & 0,3421 & Peto e & 0,3893 & 0,4926 & 0,3147 & 0,3422 & Peto e & 0,3893 & 0,4926 & 0,3147 & 0,3418 \\
\hline 200 & Harrington $\gamma=0,5$ & 0,4061 & 0,5058 & 0,3274 & 0,3571 & Peto & 0,4061 & 0,5058 & 0,3274 & 0,3572 & Peto & 0,4061 & 0,5058 & 0,3274 & 0,3573 \\
\hline 220 & $\rho=2$ & 0,4433 & 0,5414 & 0,3605 & 0,393 & & 0,4433 & 0,5414 & 0,3605 & 0,393 & & 0,4433 & 0,5414 & 0,3605 & 0,393 \\
\hline 250 & $\rho=2$ & 0,4976 & 0,5991 & 0,4013 & 0,4423 & & 0,4976 & 0,5991 & 0,4013 & 0,4443 & & 0,4976 & 0,5993 & 0,4013 & 0,4404 \\
\hline 300 & & 0,5927 & 0,6931 & 0,4925 & 0,5333 & & 0,5927 & 0,6931 & 0,4925 & 0,5332 & & 0,5927 & 0,6932 & 0,4925 & 0,5333 \\
\hline 350 & & 0,6662 & 0,7602 & 0,5638 & 0,6091 & & 0,6662 & 0,7602 & 0,5638 & 0,6092 & & 0,6662 & 0,7602 & 0,5638 & 0,6087 \\
\hline 400 & & 0,7274 & 0,8183 & 0,6314 & 0,6752 & & 0,7274 & 0,8183 & 0,6314 & 0,6753 & & 0,7274 & 0,8183 & 0,6314 & 0,6751 \\
\hline 450 & & 0,7865 & 0,8624 & 0,695 & 0,7372 & & 0,7865 & 0,8624 & 0,695 & 0,737 & & 0,7865 & 0,8624 & 0,6951 & 0,7366 \\
\hline 500 & & 0,8356 & 0,8994 & 0,7511 & 0,7872 & & 0,8356 & 0,8994 & 0,7511 & 0,7873 & & 0,8356 & 0,8994 & 0,7511 & 0,7869 \\
\hline 600 & & 0,9033 & 0,9466 & 0,8404 & 0,8723 & & 0,9033 & 0,9466 & 0,8404 & 0,8732 & & 0,9033 & 0,9466 & 0,8404 & 0,8701 \\
\hline 700 & & 0,9448 & 0,9719 & 0,9065 & 0,927 & & 0,9448 & 0,9719 & 0,9065 & 0,927 & & 0,9448 & 0,9719 & 0,9065 & 0,9265 \\
\hline
\end{tabular}

Tabela 4.7: Função Poder do teste para 5 populações, $\delta$ de 0,05 e sem censura.

Peso: Log-rank

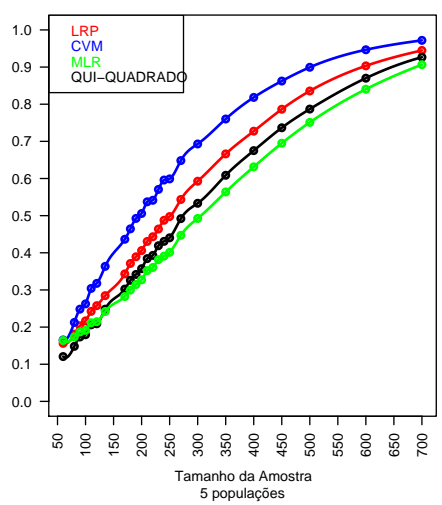

Peso: Fleming e Harrington, Gamma=0.5,Rho=2

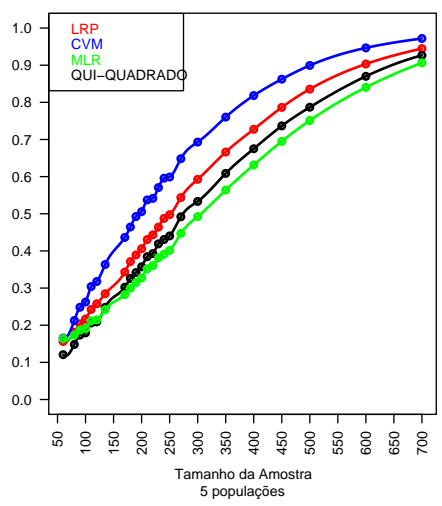

Peso: Gehan

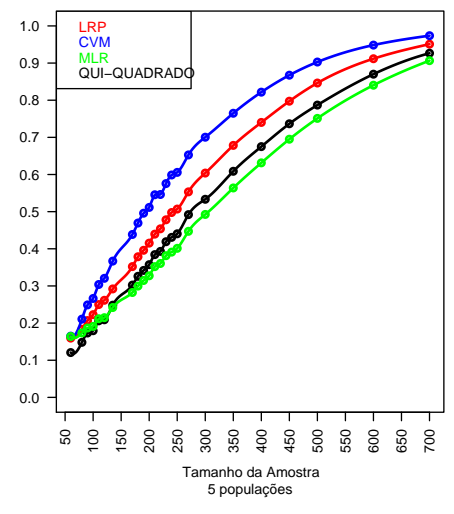

Peso: Peto e Peto

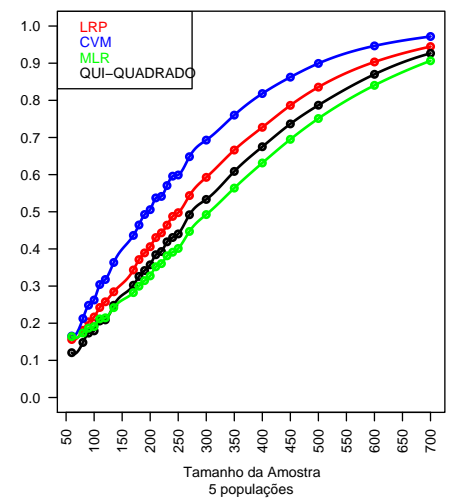

Peso: Tarone e Ware, Gamma $=0.5$

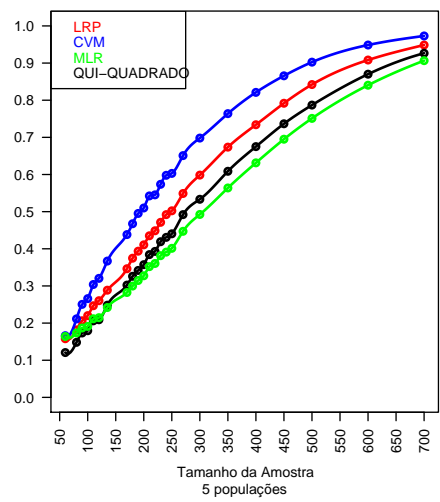

Peso: Peto e Peto Modificado

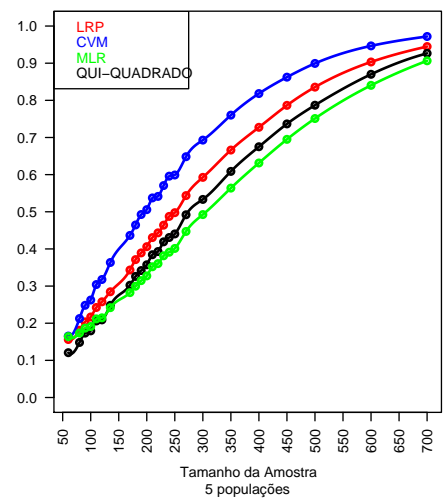

Figura 4.8: Gráfico da Função Poder do teste para 5 populações, $\delta$ de 0,05 e sem censura. 
Resumindo os resultados, observamos que a estatística de Cramér-von Mises, assim como no caso anterior, têm comportamento melhor que as demais nas três situações. Como no caso anterior, temos que as estatística de MLR e Qui-quadrado têm o mesmo comportamento nas três situações e têm o poder um pouco menor que o CVM. Por fim, notamos que a estatística de LRP têm um poder similar ao CVM para duas populações e o poder vêm diminuindo quando aumentamos o número de populações.

\subsubsection{Simulação do poder com 1 cruzamento nas funções intensidade}

Nosso objetivo agora é avaliar a função poder do teste no caso em que ocorre cruzamento das funções de intensidade das populações. Para isto, realizamos um experimento no qual a hipótese alternativa com cruzamento das funções intensidade é fixada, sendo variado o tamanho da amostra. No experimento, os tamanhos de amostra escolhidos para a simulação são 80, 100, 150, 200, 250 e 300, já para as funções de ponderação, usamos todas descritas na tabela (3.1). A seguir apresentamos os passos do algoritmo.

1. Fixamos um nível de significância $\alpha=0,05$;

2. Definimos J populações com distribuição Multinomial:

- $X^{(1)}, \ldots, X^{(J)} \sim M\left(T A, p_{1}, p_{2}, p_{3}, p_{4}, p_{5}, p_{6}\right)$

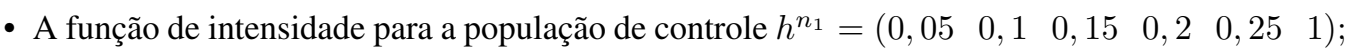

- A função de intensidade para as populações de tratamento é a função constante, ou seja $h^{n_{2}}=$ $\cdots=h^{n_{J}}=\left(\begin{array}{llllll}0,15 & 0,15 & 0,15 & 0,15 & 0,15 & 1\end{array}\right)$;

- À partir das funções de intensidade, calculamos as probabilidade $p_{1}, \ldots, p_{J}$ usando a equação (2.5), ou seja, $p_{i}=P[W=i]=h(i) \prod_{\ell=1}^{i-1}[1-h(\ell)]$ dado que a hipótese nula é falsa;

- Escolhemos uma das funções de ponderação descrita na tabela (3.1);

3. Realizamos o teste de hipótese usando valor crítico ao nível de significância $\alpha$;

4. Para estimarmos a função poder tomamos

$$
y_{m}= \begin{cases}1, & \text { se } H_{0} \text { foi rejeitada ao nível de significância } \alpha \\ 0, & \text { caso contrário }\end{cases}
$$

5. Repetimos as etapas 2 a $4 m=10000$ vezes;

6. Estimamos o poder do teste na seguinte forma:

$$
\text { Poder }=\frac{1}{m} \sum_{k=1}^{m} y_{k}
$$




\begin{tabular}{|c|c|c|c|c|c|c|c|c|c|c|c|c|c|c|c|}
\hline \multirow[t]{2}{*}{ TA } & \multirow[b]{2}{*}{ Peso } & \multicolumn{4}{|c|}{ Estatística } & \multicolumn{5}{|c|}{ Estatística } & \multicolumn{5}{|c|}{ Estatística } \\
\hline & & LRP & CVM & MLR & QQ & Peso & LRP & CVM & MLR & QQ & Peso & LRP & CVM & MLR & QQ \\
\hline 80 & & 0,0892 & 0,3348 & 0,5285 & 0,4931 & & 0,1816 & 0,5134 & 0,5285 & 0,4931 & & 0,1295 & 0,4371 & 0,5285 & 0,4931 \\
\hline 100 & & 0,0927 & 0,4107 & 0,6506 & 0,6253 & & 0,2114 & 0,6106 & 0,6506 & 0,6253 & & 0,1441 & 0,5258 & 0,6506 & 0,6253 \\
\hline 150 & Log-rank & 0,1122 & 0,5871 & 0,8438 & 0,8336 & Gehan & 0,2898 & 0,7991 & 0,8438 & 0,8336 & Tarone e & 0,1919 & 0,7156 & 0,8438 & 0,8336 \\
\hline 200 & & 0,1418 & 0,726 & 0,9372 & 0,9332 & & 0,3631 & 0,8909 & 0,9372 & 0,9332 & Ware & 0,2407 & 0,8315 & 0,9372 & 0,9332 \\
\hline 250 & & 0,1615 & 0,8276 & 0,9745 & 0,9729 & & 0,4351 & 0,9485 & 0,9745 & 0,9729 & $\gamma=0,5$ & 0,2879 & 0,9066 & 0,9745 & 0,9729 \\
\hline 300 & & 0,1844 & 0,9055 & 0,9937 & 0,9934 & & 0,5137 & 0,9768 & 0,9937 & 0,9934 & & 0,3379 & 0,9561 & 0,9937 & 0,9934 \\
\hline 80 & & 0,1111 & 0,3593 & 0,5285 & 0,4931 & & 0,1574 & 0,4761 & 0,5285 & 0,4931 & & 0,1578 & 0,4768 & 0,5285 & 0,4931 \\
\hline 100 & & 0,1163 & 0,4368 & 0,6506 & 0,6253 & & 0,1779 & 0,5691 & 0,6506 & 0,6253 & & 0,1784 & 0,5696 & 0,6506 & 0,6253 \\
\hline 150 & Fleming e & 0,1482 & 0,6073 & 0,8438 & 0,8336 & Peto e & 0,249 & 0,7615 & 0,8438 & 0,8336 & Peto e & 0,2494 & 0,7617 & 0,8438 & 0,8336 \\
\hline 200 & Harrington & 0,183 & 0,7385 & 0,9372 & 0,9332 & Peto & 0,3122 & 0,8651 & 0,9372 & 0,9332 & Peto & 0,3125 & 0,8654 & 0,9372 & 0,9332 \\
\hline 250 & $\gamma=0,5 \mathrm{e}$ & 0,2169 & 0,8322 & 0,9745 & 0,9729 & & 0,3734 & 0,9316 & 0,9745 & 0,9729 & Modificado & 0,3739 & 0,9316 & 0,9745 & 0,9729 \\
\hline 300 & $\rho=2$ & 0,2458 & 0,9057 & 0,9937 & 0,9934 & & 0,4427 & 0,9693 & 0,9937 & 0,9934 & & 0,4432 & 0,9694 & 0,9937 & 0,9934 \\
\hline
\end{tabular}

Tabela 4.8: Função Poder do teste para 2 populações e sem censura.
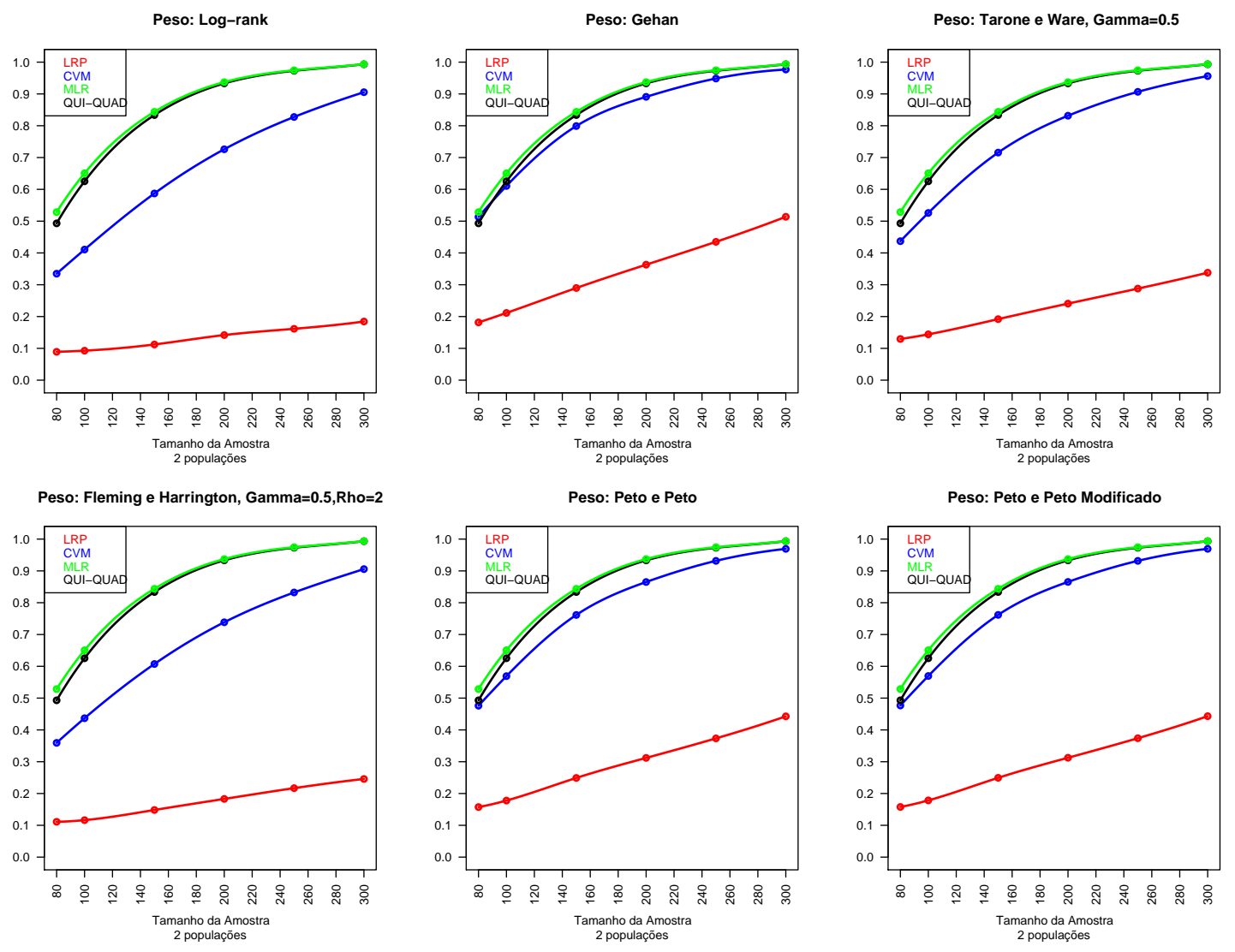

Figura 4.9: Gráfico da Função Poder do teste para 2 populações e sem censura.

Nos experimentos para cruzamento das funções intensidade e 2 populações temos que as duas funções de intensidade cruzam no $\ell=3$. Notamos que as variáveis de interesse $W^{n_{1}}$ e $W^{n_{2}}$ tem 27,3\% (30\%) de chance de estar abaixo ou igual a 2 e 72,7\% (70\%) de chance de estar localizado após ou igual ao ponto de cruzamento $\ell=3$. Assim, pela tabela (4.8), notamos que o teste de Log-rank ponderado têm poder muito baixo e a modificação do teste de Log-rank nos proporciona o melhor poder para dados finitos e com cruzamento das funções de intensidade. Note também que para a função de ponderação Gehan o teste de Cramér-von Mises têm poder similar aos testes de Log-rank modificado e Qui-quadrado. 


\begin{tabular}{|c|c|c|c|c|c|c|c|c|c|c|c|c|c|c|c|}
\hline \multirow{2}{*}{ TA } & \multirow[b]{2}{*}{ Peso } & \multicolumn{4}{|c|}{ Estatística } & \multicolumn{5}{|c|}{ Estatística } & \multicolumn{5}{|c|}{ Estatística } \\
\hline & & LRP & CVM & MLR & QQ & Peso & LRP & CVM & MLR & QQ & Peso & LRP & CVM & MLR & QQ \\
\hline 80 & & 0,0826 & 0,364 & 0,6094 & 0,4871 & & 0,1816 & 0,5552 & 0,6094 & 0,4871 & & 0,1212 & 0,4758 & 0,6094 & 0,4871 \\
\hline 100 & & 0,0938 & 0,4537 & 0,7172 & 0,6089 & & 0,2267 & 0,6652 & 0,7172 & 0,6089 & & 0,1456 & 0,5804 & 0,7172 & 0,6089 \\
\hline 150 & Log-rank & 0,1027 & 0,6376 & 0,8902 & 0,8375 & Gehan & 0,2941 & 0,8436 & 0,8902 & 0,8375 & Tarone e & 0,1809 & 0,7659 & 0,8902 & 0,8375 \\
\hline 200 & & 0,1332 & 0,7918 & 0,9651 & 0,9448 & & 0,3951 & 0,9341 & 0,9651 & 0,9448 & Ware & 0,2434 & 0,8895 & 0,9651 & 0,9448 \\
\hline 250 & & 0,1454 & 0,8861 & 0,9914 & 0,9841 & & 0,4606 & 0,9772 & 0,9914 & 0,9841 & $\gamma=0,5$ & 0,2821 & 0,9539 & 0,9914 & 0,9841 \\
\hline 300 & & 0,1632 & 0,9481 & 0,9978 & 0,9969 & & 0,5487 & 0,992 & 0,9978 & 0,9969 & & 0,3376 & 0,9811 & 0,9978 & 0,9969 \\
\hline 80 & & 0,1118 & 0,4242 & 0,6094 & 0,4871 & & 0,1514 & 0,5163 & 0,6094 & 0,4871 & & 0,1516 & 0,5168 & 0,6094 & 0,4871 \\
\hline 100 & & 0,127 & 0,5157 & 0,7172 & 0,6089 & & 0,1879 & 0,6259 & 0,7172 & 0,6089 & & 0,1882 & 0,6265 & 0,7172 & 0,6089 \\
\hline 150 & Fleming e & 0,1532 & 0,6967 & 0,8902 & 0,8375 & Peto e & 0,2398 & 0,8069 & 0,8902 & 0,8375 & Peto e & 0,2423 & 0,8072 & 0,8902 & 0,8375 \\
\hline 200 & Harrington & 0,2045 & 0,8373 & 0,9651 & 0,9448 & Peto & 0,3221 & 0,9112 & 0,9651 & 0,9448 & Peto & 0,3224 & 0,9115 & 0,9651 & 0,9448 \\
\hline 250 & $\gamma=0,5 \mathrm{e}$ & 0,2317 & 0,9173 & 0,9914 & 0,9841 & & 0,3812 & 0,9672 & 0,9914 & 0,9841 & Modificado & 0,3811 & 0,9672 & 0,9914 & 0,9841 \\
\hline 300 & $\rho=2$ & 0,2717 & 0,9623 & 0,9978 & 0,9969 & & 0,4508 & 0,9876 & 0,9978 & 0,9969 & & 0,4511 & 0,9876 & 0,9978 & 0,9969 \\
\hline
\end{tabular}

Tabela 4.9: Função Poder do teste para 3 populações e sem censura.
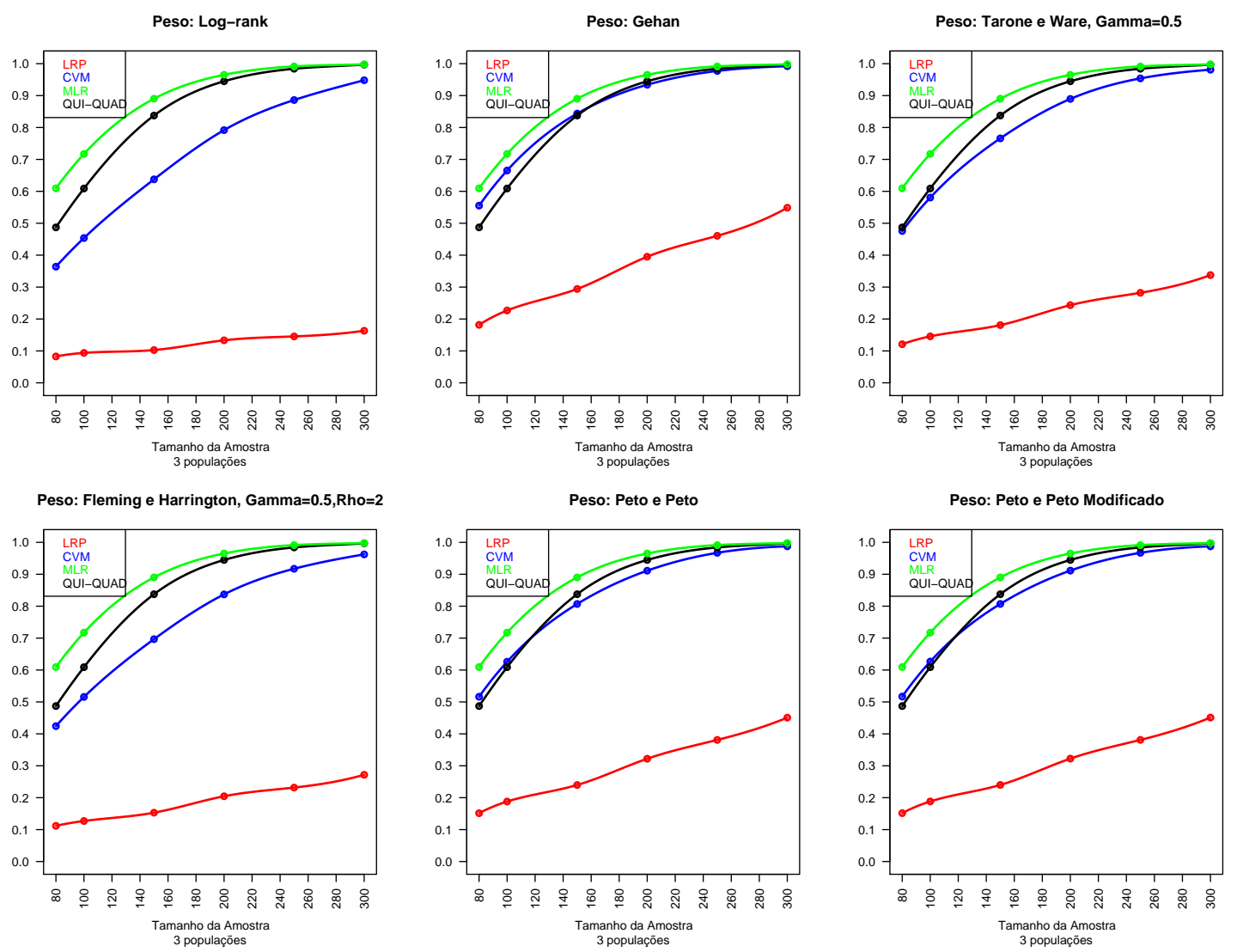

Figura 4.10: Gráfico da Função Poder do teste para 3 populações e sem censura.

Nos experimentos para cruzamento das funções de intensidade e 3 populações, pela tabela (4.9), notamos que o teste de Log-rank ponderado, assim como no caso de 2 populações, tem poder muito baixo e a modificação do teste de Log-rank nos proporciona o melhor poder para dados finitos e com cruzamento das funções de intensidade. Note também que o poder para o teste Qui-Quadrado diminui em relação ao caso de 2 populações quando inserimos mais uma população (para tamanhos de amostra pequenos) e que para a função de ponderação Gehan o teste de Cramér-von Mises têm poder maior que o teste Qui-Quadrado. 


\begin{tabular}{|c|c|c|c|c|c|c|c|c|c|c|c|c|c|c|c|}
\hline \multirow[t]{2}{*}{ TA } & \multirow[b]{2}{*}{ Peso } & \multicolumn{4}{|c|}{ Estatística } & \multicolumn{5}{|c|}{ Estatística } & \multicolumn{5}{|c|}{ Estatística } \\
\hline & & LRP & CVM & MLR & QQ & Peso & LRP & CVM & MLR & QQ & Peso & LRP & CVM & MLR & QQ \\
\hline 80 & & 0,0824 & 0,2589 & 0,652 & 0,4207 & & 0,1871 & 0,3962 & 0,6521 & 0,4207 & & 0,1231 & 0,3398 & 0,652 & 0,4207 \\
\hline 100 & & 0,084 & 0,3373 & 0,7306 & 0,5345 & & 0,2219 & 0,5181 & 0,7306 & 0,5345 & & 0,1395 & 0,4452 & 0,7306 & 0,5345 \\
\hline 150 & Log-rank & 0,102 & 0,5364 & 0,8942 & 0,7845 & Gehan & 0,3109 & 0,7708 & 0,8942 & 0,7845 & Tarone e & 0,1871 & 0,6852 & 0,8942 & 0,7845 \\
\hline 200 & & 0,1185 & 0,7233 & 0,9676 & 0,9194 & & 0,4163 & 0,9151 & 0,9676 & 0,9194 & Ware & 0,243 & 0,8529 & 0,9676 & 0,9194 \\
\hline 250 & & 0,1395 & 0,8356 & 0,9908 & 0,9767 & & 0,5007 & 0,9684 & 0,9908 & 0,9767 & $\gamma=0,5$ & 0,2957 & 0,9322 & 0,9908 & 0,9767 \\
\hline 300 & & 0,1704 & 0,9232 & 0,9978 & 0,9935 & & 0,5935 & 0,9923 & 0,9978 & 0,9935 & & 0,3526 & 0,978 & 0,9978 & 0,9935 \\
\hline 80 & & 0,1148 & 0,3166 & 0,652 & 0,4207 & & 0,1539 & 0,3676 & 0,6522 & 0,4207 & & 0,1542 & 0,3679 & 0,652 & 0,4207 \\
\hline 100 & & 0,1291 & 0,4122 & 0,7306 & 0,5345 & & 0,1796 & 0,4833 & 0,7306 & 0,5345 & & 0,1799 & 0,4835 & 0,7306 & 0,5345 \\
\hline 150 & Fleming e & 0,1685 & 0,638 & 0,8942 & 0,7845 & Peto e & 0,2452 & 0,7257 & 0,8942 & 0,7845 & Peto e & 0,2456 & 0,7257 & 0,8942 & 0,7845 \\
\hline 200 & Harrington & 0,2184 & 0,8128 & 0,9676 & 0,9194 & Peto & 0,3283 & 0,8842 & 0,9676 & 0,9194 & Peto & 0,3287 & 0,8844 & 0,9676 & 0,9194 \\
\hline 250 & $\gamma=0,5 \mathrm{e}$ & 0,2607 & 0,9043 & 0,9908 & 0,9767 & & 0,4005 & 0,9518 & 0,9908 & 0,9767 & Modificado & 0,4008 & 0,9518 & 0,9908 & 0,9767 \\
\hline 300 & $\rho=2$ & 0,3132 & 0,9632 & 0,9978 & 0,9935 & & 0,4751 & 0,985 & 0,9978 & 0,9935 & & 0,4754 & 0,9853 & 0,9978 & 0,9935 \\
\hline
\end{tabular}

Tabela 4.10: Função Poder do teste para 5 populações e sem censura.
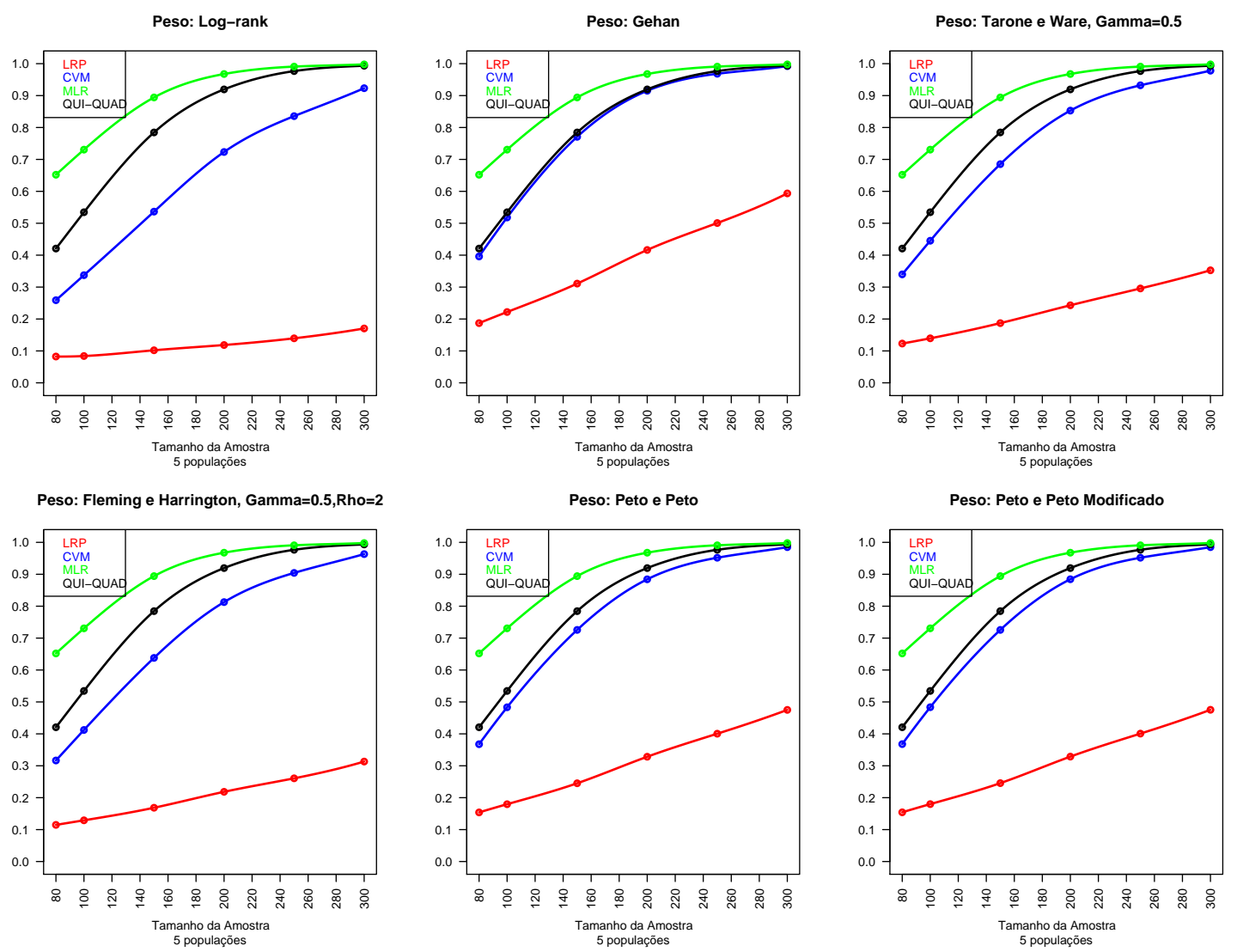

Figura 4.11: Gráfico da Função Poder do teste para 5 populações e sem censura.

Nos experimentos para cruzamento das funções de intensidade e 5 populações, pela tabela (4.10), notamos que o teste de Log-rank ponderado, assim como no caso de 2 e 3 populações, têm poder muito baixo e a modificação do teste de Log-rank nos proporciona o melhor poder para dados finitos e com cruzamento das funções de intensidade. Note também que o poder para o teste Qui-Quadrado diminui em relação ao caso de 3 populações quando inserimos mais duas populações (para tamanhos de amostra pequenos). Resumindo as três situações temos que o teste de Log-rank modificado têm o maior poder em relações a todos os testes estudados e que esse poder vêm aumentando quando aumentamos o número de populações. 


\subsubsection{Simulação do poder do teste com 2 cruzamentos das funções de intensidade}

O último experimento para espaços amostrais finitos é o caso de dois cruzamentos das funções de intensidade. Para este experimento, usamos duas e três populações, para duas usamos as funções de intensidade como mostrado na figura (4.12) e para três populações repetimos a função de intensidade constante usada para a segunda população. Com respeito aos processos de ponderação, usamos todos os processos descritos na tabela (3.1). Assim, estudamos quatro situações, dois experimentos para duas populações (sem e com censura) e dois experimentos para três populações (com e sem censura). No caso sem censura, comparamos Cramér-von Mises (CVM), Log-rank Ponderado (LRP) e Log-rank Modificado (MLR) com a estatística Qui-Quadrado (QQ).

1. Fixamos um nível de significância $\alpha=0,05$;

2. Definimos J populações com distribuição Multinomial:

- $X^{(1)}, \ldots, X^{(J)} \sim M\left(T A, p_{1}, p_{2}, \ldots, p_{20}\right)$

- No caso com censura, temos $C^{(1)}, \ldots, C^{(J)} \sim U[1,20]$ truncado;

- A função de intensidade para a população de controle $h^{n_{1}}$ e a função de intensidade para as populações de tratamento é a função constante, ou seja,$h^{n_{2}}=\cdots=h^{n_{J}}$;

- À partir das funções de intensidade, calculamos as probabilidade $p_{1}, \ldots, p_{19}$ e $p_{20}$ usando a equação (2.5), ou seja, $p_{i}=P[W=i]=h(i) \prod_{\ell=1}^{i-1}[1-h(\ell)]$ dado que a hipótese nula é falsa;

- Escolhemos uma das funções de ponderação descrita na tabela (3.1);

3. Realizamos o teste de hipótese usando valor crítico ao nível de significância $\alpha$;

4. Para estimarmos a função poder tomamos

$$
y_{m}= \begin{cases}1, & \text { se } H_{0} \text { foi rejeitada ao nível de significância } \alpha \\ 0, & \text { caso contrário }\end{cases}
$$

5. Repetimos as etapas 2 a $4 m=10000$ vezes;

6. Estimamos o poder do teste na seguinte forma:

$$
\text { Poder }=\frac{1}{m} \sum_{k=1}^{m} y_{k}
$$

Neste primeiro experimento, observamos que o teste de MLR tem melhor desempenho para qualquer função de ponderação, em seguida, temos o teste Qui-Quadrado. Uma observação importante é que LRP não se aproxima de 1 mesmo para amostras grandes. 


\section{Taxas de Falha}

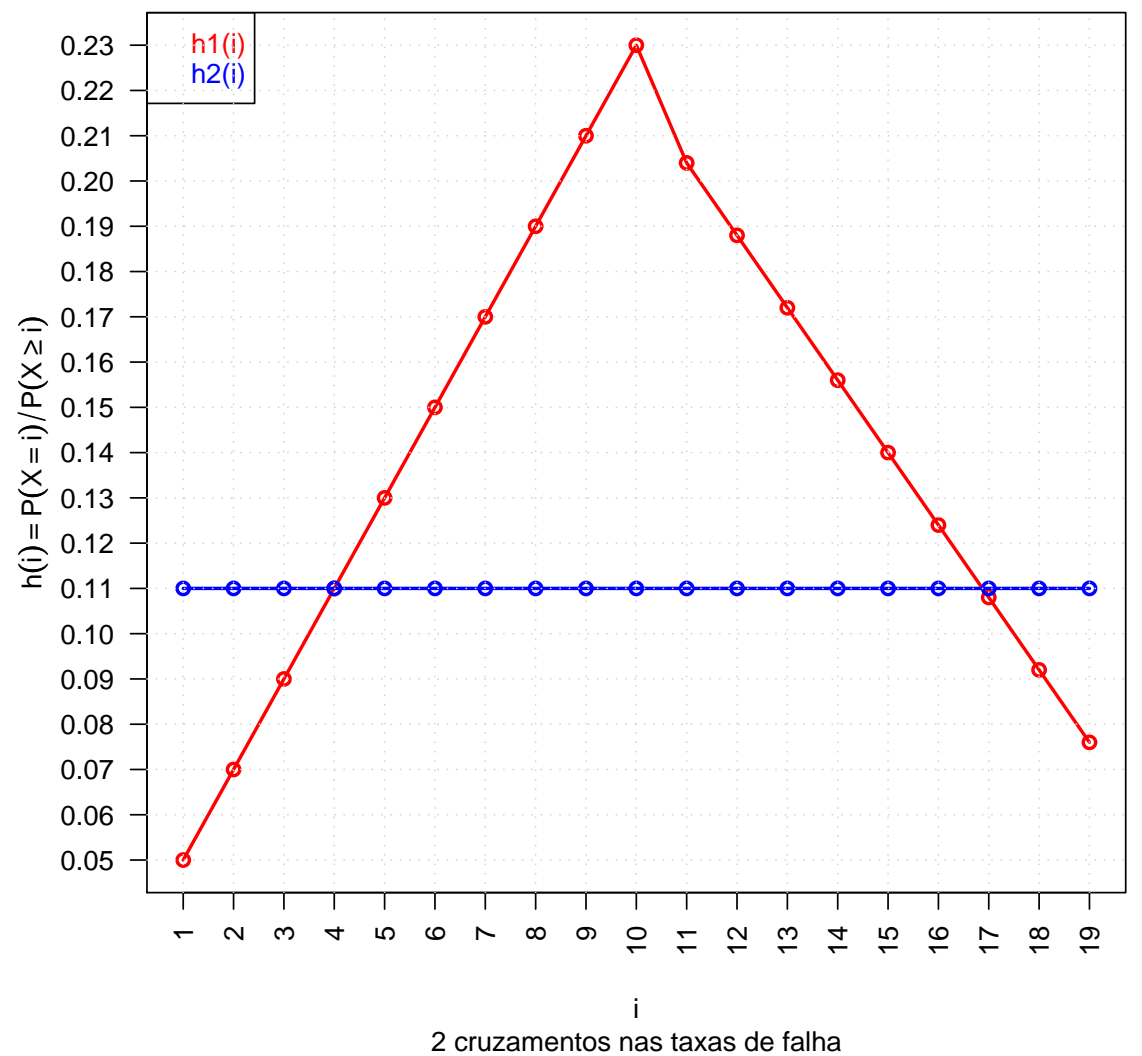

Figura 4.12: Gráfico para 2 cruzamentos em espaços amostrais finitos.

\begin{tabular}{|c|c|c|c|c|c|c|c|c|c|c|c|c|c|c|c|c|c|c|c|c|}
\hline$\ell$ & 1 & 2 & 3 & 4 & 5 & 6 & 7 & 8 & 9 & 10 & 11 & 12 & 13 & 14 & 15 & 16 & 17 & 18 & 19 & 20 \\
\hline$h^{1}(\ell)$ & 0,05 & 0,07 & 0,09 & 0,11 & 0,13 & 0,15 & 0,17 & 0,19 & 0,21 & 0,23 & 0,204 & 0,188 & 0,172 & 0,156 & 0,14 & 0,124 & 0,108 & 0,092 & 0,076 & 1 \\
\hline$h^{2}(\ell)$ & 0,11 & 0,11 & 0,11 & 0,11 & 0,11 & 0,11 & 0,11 & 0,11 & 0,11 & 0,11 & 0,11 & 0,11 & 0,11 & 0,11 & 0,11 & 0,11 & 0,11 & 0,11 & 0,11 & 1 \\
\hline$h^{3}(\ell)$ & 0,11 & 0,11 & 0,11 & 0,11 & 0,11 & 0,11 & 0,11 & 0,11 & 0,11 & 0,11 & 0,11 & 0,11 & 0,11 & 0,11 & 0,11 & 0,11 & 0,11 & 0,11 & 0,11 & 1 \\
\hline
\end{tabular}

Tabela 4.11: Funções de intensidade para experimentos de 2 cruzamentos.

\begin{tabular}{|c|c|c|c|c|c|c|c|c|c|c|c|c|c|c|c|}
\hline TA & Peso & LRP & CVM & MLR & QQ & Peso & LRP & CVM & MLR & QQ & Peso & LRP & CVM & MLR & QQ \\
\hline 60 & & 0,1105 & 0,0825 & 0,4183 & 0,2029 & & 0,0594 & 0,1582 & 0,4183 & 0,2029 & & 0,0549 & 0,2144 & 0,4183 & 0,2029 \\
\hline 80 & & 0,1509 & 0,1083 & 0,5005 & 0,3025 & & 0,0731 & 0,1932 & 0,5005 & 0,3025 & & 0,0619 & 0,2726 & 0,5005 & 0,3025 \\
\hline 100 & & 0,1672 & 0,1257 & 0,5714 & 0,4038 & & 0,0709 & 0,2307 & 0,5714 & 0,4038 & & 0,0603 & 0,3228 & 0,5714 & 0,4038 \\
\hline 150 & & 0,2241 & 0,1822 & 0,7435 & 0,6483 & & 0,0768 & 0,3295 & 0,7435 & 0,6483 & & 0,0523 & 0,4642 & 0,7435 & 0,6481 \\
\hline 200 & & 0,2804 & 0,2867 & 0,8632 & 0,8193 & & 0,0832 & 0,4526 & 0,8612 & 0,8193 & & 0,0564 & 0,5917 & 0,8632 & 0,8193 \\
\hline 250 & Log-Rank & 0,3427 & 0,4189 & 0,9358 & 0,9187 & Tarone e Ware & 0,0935 & 0,5532 & 0,9358 & 0,9187 & Peto e Peto & 0,0545 & 0,6916 & 0,9358 & 0,9187 \\
\hline 300 & & 0,3981 & 0,5704 & 0,9719 & 0,9697 & $\gamma=0,5$ & 0,1003 & 0,6601 & 0,9719 & 0,9697 & & 0,0586 & 0,7747 & 0,9719 & 0,9697 \\
\hline 350 & & 0,4527 & 0,7099 & 0,9899 & 0,9882 & & 0,1149 & 0,7491 & 0,9899 & 0,9883 & & 0,0585 & 0,8409 & 0,9899 & 0,9882 \\
\hline 400 & & 0,5112 & 0,8163 & 0,9971 & 0,9956 & & 0,1125 & 0,8273 & 0,9971 & 0,9956 & & 0,0554 & 0,8946 & 0,9971 & 0,9956 \\
\hline 450 & & 0,5546 & 0,8998 & 0,9989 & 0,9976 & & 0,1235 & 0,8819 & 0,9989 & 0,9976 & & 0,0584 & 0,9277 & 0,9989 & 0,9976 \\
\hline 500 & & 0,5942 & 0,9444 & 0,9996 & 0,9995 & & 0,1374 & 0,9219 & 0,9996 & 0,9995 & & 0,0578 & 0,9532 & 0,9996 & 0,9995 \\
\hline 60 & & 0,0569 & 0,2267 & 0,4183 & 0,2029 & & 0,0576 & 0,1691 & 0,4183 & 0,2029 & & 0,0553 & 0,2156 & 0,4183 & 0,2029 \\
\hline 80 & & 0,0633 & 0,2872 & 0,5005 & 0,3025 & & 0,0669 & 0,2098 & 0,5005 & 0,3025 & & 0,0621 & 0,2739 & 0,5005 & 0,3025 \\
\hline 100 & & 0,0638 & 0,3416 & 0,5714 & 0,4038 & & 0,0668 & 0,245 & 0,5714 & 0,4038 & & 0,0604 & 0,3235 & 0,5714 & 0,4038 \\
\hline 150 & & 0,0546 & 0,4863 & 0,7435 & 0,6482 & & 0,0576 & 0,3443 & 0,7435 & 0,6484 & & 0,0524 & 0,4652 & 0,7435 & 0,6482 \\
\hline 200 & & 0,0623 & 0,6168 & 0,8643 & 0,8193 & & 0,0579 & 0,4516 & 0,8623 & 0,8193 & & 0,0566 & 0,5928 & 0,8643 & 0,8193 \\
\hline 250 & Gehan & 0,0598 & 0,7152 & 0,9358 & 0,9187 & Fleming $\mathrm{e}$ & 0,0589 & 0,5371 & 0,9358 & 0,9187 & Peto e Peto & 0,0546 & 0,6921 & 0,9358 & 0,9187 \\
\hline 300 & & 0,0652 & 0,7962 & 0,9719 & 0,9697 & Harrington & 0,0614 & 0,6272 & 0,9719 & 0,9697 & Modificado & 0,0587 & 0,7752 & 0,9719 & 0,9697 \\
\hline 350 & & 0,0662 & 0,857 & 0,9899 & 0,9882 & $\gamma=0,5 \mathrm{e}$ & 0,0627 & 0,6987 & 0,9899 & 0,9883 & & 0,0584 & 0,8411 & 0,9899 & 0,9883 \\
\hline 400 & & 0,0663 & 0,9068 & 0,9971 & 0,9956 & $\rho=2$ & 0,0593 & 0,7739 & 0,9971 & 0,9956 & & 0,0556 & 0,8948 & 0,9971 & 0,9956 \\
\hline 450 & & 0,0683 & 0,9364 & 0,9989 & 0,9976 & & 0,0608 & 0,8212 & 0,9989 & 0,9976 & & 0,0589 & 0,9279 & 0,9989 & 0,9976 \\
\hline 500 & & 0,0682 & 0,9597 & 0,9996 & 0,9995 & & 0,0624 & 0,8602 & 0,9996 & 0,9995 & & 0,0579 & 0,9532 & 0,9996 & 0,9995 \\
\hline
\end{tabular}

Tabela 4.12: Tabela da função poder do testes para 2 cruzamentos, 2 populações e sem censura. 
Peso: Log-rank

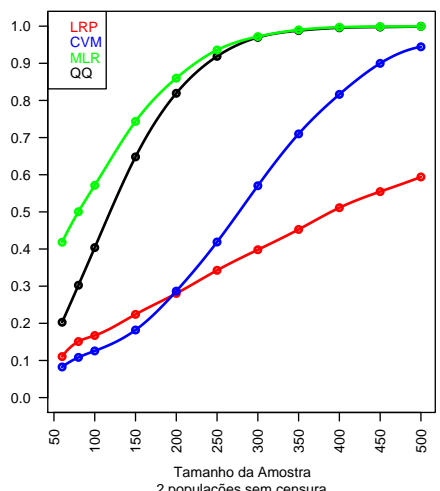

Peso: Fleming e Harrington, Gamma=0.5,Rho=2

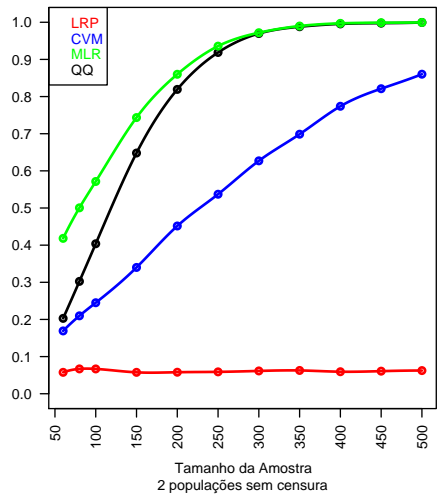

Peso: Gehan

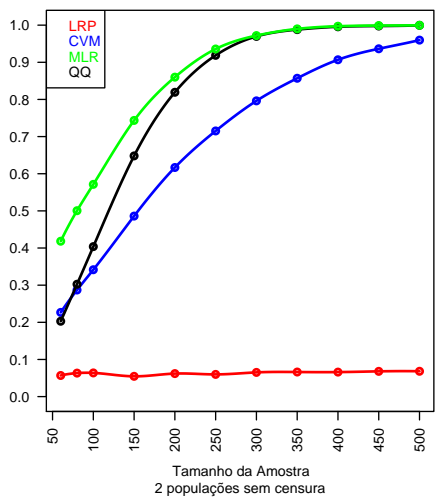

Peso: Peto e Peto

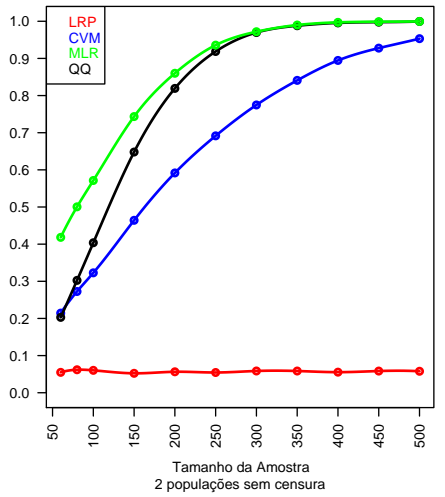

Peso: Tarone e Ware, Gamma $=0.5$

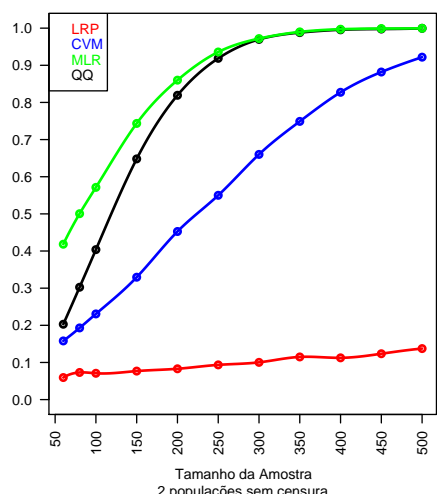

Peso: Peto e Peto Modificado

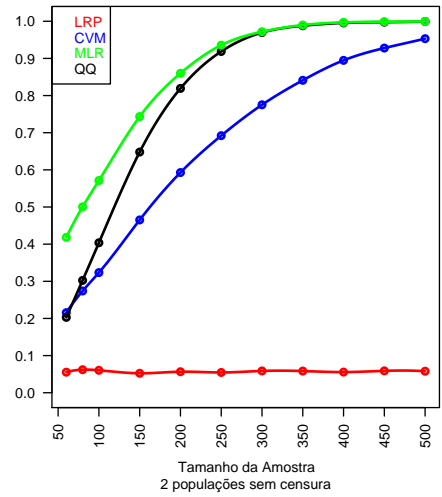

Figura 4.13: Gráfico do poder do testes para 2 cruzamentos, 2 populações e sem censura.

\begin{tabular}{|c|c|c|c|c|c|c|c|c|c|c|c|c|c|c|}
\hline TA & Peso & LRP & CVM & MLR & Peso & LRP & CVM & MLR & Peso & LRP & CVM & MLR & Cens1 \% & Cens $2 \%$ \\
\hline 60 & & 0,2226 & 0,2594 & 0,2181 & & 0,2318 & 0,2761 & 0,2181 & & 0,2355 & 0,2835 & 0,2181 & & \\
\hline 80 & & 0,2732 & 0,3311 & 0,2674 & & 0,2842 & 0,3536 & 0,2674 & & 0,2883 & 0,3624 & 0,2674 & & \\
\hline 100 & & 0,3276 & 0,3968 & 0,3282 & & 0,3411 & 0,4253 & 0,3282 & & 0,3468 & 0,4363 & 0,3282 & & \\
\hline 150 & & 0,4452 & 0,5395 & 0,4727 & & 0,4641 & 0,5746 & 0,4727 & & 0,4744 & 0,5931 & 0,4727 & & \\
\hline 200 & & 0,5554 & 0,6709 & 0,6022 & & 0,5784 & 0,7052 & 0,6022 & & 0,5914 & 0,7212 & 0,6022 & & \\
\hline 250 & Log-Rank & 0,6278 & 0,7566 & 0,7012 & Tarone e Ware & 0,6552 & 0,7963 & 0,7012 & Peto e Peto & 0,6692 & 0,8132 & 0,7012 & 54,86 & 58,23 \\
\hline 300 & & 0,6963 & 0,8301 & 0,7888 & $\gamma=0,5$ & 0,7243 & 0,8634 & 0,7888 & & 0,7393 & 0,8769 & 0,7888 & & \\
\hline 350 & & 0,7467 & 0,8757 & 0,8411 & & 0,7767 & 0,9071 & 0,8411 & & 0,7939 & 0,9198 & 0,8411 & & \\
\hline 400 & & 0,7877 & 0,9096 & 0,8922 & & 0,8157 & 0,934 & 0,8923 & & 0,8316 & 0,9438 & 0,8921 & & \\
\hline 450 & & 0,8156 & 0,9413 & 0,9264 & & 0,8467 & 0,9615 & 0,9264 & & 0,8664 & 0,9685 & 0,9264 & & \\
\hline 500 & & 0,8439 & 0,9624 & 0,9566 & & 0,8721 & 0,9751 & 0,9566 & & 0,8939 & 0,9813 & 0,9566 & & \\
\hline 60 & & 0,2391 & 0,2881 & 0,2181 & & 0,2185 & 0,2537 & 0,2181 & & 0,2356 & 0,2839 & 0,2181 & & \\
\hline 80 & & 0,2961 & 0,3689 & 0,2674 & & 0,2663 & 0,3212 & 0,2674 & & 0,2884 & 0,3626 & 0,2674 & & \\
\hline 100 & & 0,3548 & 0,4441 & 0,3282 & & 0,3176 & 0,3809 & 0,3282 & & 0,3468 & 0,4361 & 0,3282 & & \\
\hline 150 & & 0,4843 & 0,6022 & 0,4727 & & 0,4312 & 0,5225 & 0,4727 & & 0,4745 & 0,5932 & 0,4727 & & \\
\hline 200 & & 0,6037 & 0,7343 & 0,6022 & & 0,5379 & 0,6522 & 0,6022 & & 0,5914 & 0,7213 & 0,6022 & & \\
\hline 250 & Gehan & 0,6828 & 0,8201 & 0,7012 & Fleming e & 0,6119 & 0,7424 & 0,7012 & Peto e Peto & 0,6693 & 0,8101 & 0,7012 & 54,86 & 58,23 \\
\hline 300 & & 0,7513 & 0,8837 & 0,7888 & Harrington & 0,6801 & 0,8176 & 0,7888 & Modificado & 0,7394 & 0,8769 & 0,7888 & & \\
\hline 350 & & 0,8032 & 0,9246 & 0,8411 & $\gamma=0,5 \mathrm{e}$ & 0,7296 & 0,8662 & 0,8411 & & 0,7939 & 0,9243 & 0,8411 & & \\
\hline 400 & & 0,8424 & 0,9464 & 0,8924 & $\rho=2$ & 0,7735 & 0,9012 & 0,8921 & & 0,8317 & 0,9438 & 0,8922 & & \\
\hline 450 & & 0,8765 & 0,9714 & 0,9264 & & 0,7999 & 0,9327 & 0,9264 & & 0,8664 & 0,9685 & 0,9264 & & \\
\hline 500 & & 0,9021 & 0,9824 & 0,9566 & & 0,8327 & 0,9556 & 0,9566 & & 0,8939 & 0,9812 & 0,9566 & & \\
\hline
\end{tabular}

Tabela 4.13: Tabela da função poder do testes para 2 cruzamentos, 2 populações e com censura.

No segundo experimento, observamos que o teste de CVM têm desempenho um pouco melhor frente aos demais testes para qualquer função de ponderação. Notamos também que para o teste de LRP já detecta diferenças nas funções de intensidade quando censuramos os dados. 
Peso: Log-rank

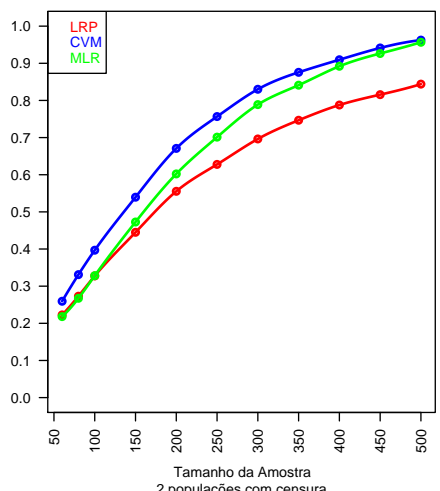

Peso: Fleming e Harrington, Gamma=0.5,Rho=2

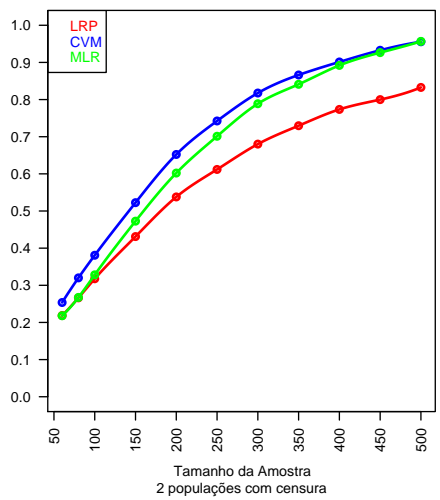

Peso: Gehan

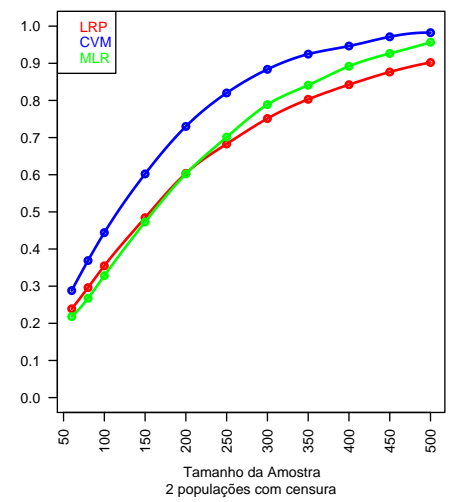

Peso: Peto e Peto

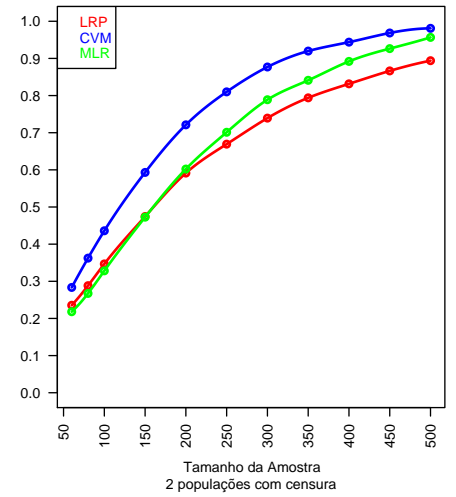

Peso: Tarone e Ware, Gamma= 0.5

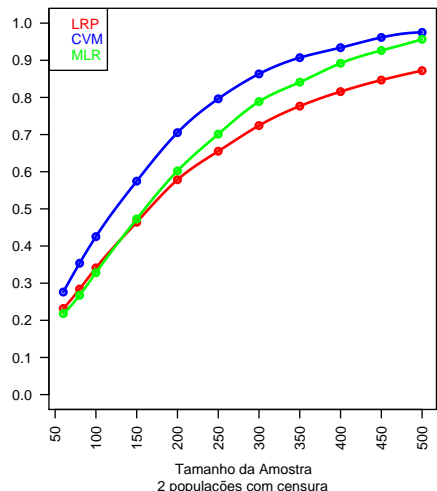

Peso: Peto e Peto Modificado

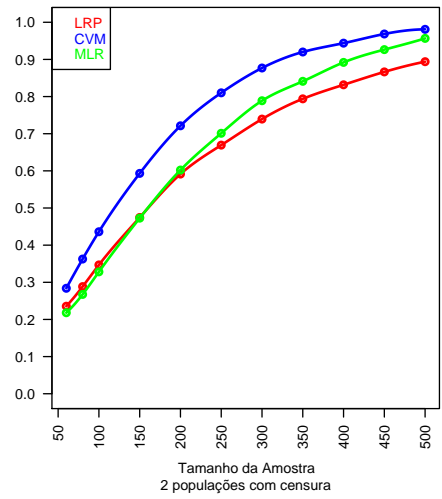

Figura 4.14: Gráfico do poder do testes para 2 cruzamentos, 2 populações e com censura.

\begin{tabular}{|c|c|c|c|c|c|c|c|c|c|c|c|c|c|c|c|}
\hline TA & Peso & LRP & CVM & MLR & $\mathrm{QQ}$ & Peso & LRP & CVM & MLR & QQ & Peso & LRP & CVM & MLR & QQ \\
\hline 60 & & 0,1024 & 0,0772 & 0,2611 & 0,2018 & & 0,0619 & 0,1504 & 0,2611 & 0,2018 & & 0,0532 & 0,2126 & 0,2611 & 0,2018 \\
\hline 80 & & 0,1302 & 0,0987 & 0,2807 & 0,3039 & & 0,0659 & 0,1956 & 0,2807 & 0,3039 & & 0,0563 & 0,2763 & 0,2807 & 0,3039 \\
\hline 100 & & 0,1421 & 0,114 & 0,3054 & 0,3953 & & 0,0641 & 0,2433 & 0,3054 & 0,3953 & & 0,0512 & 0,3448 & 0,3054 & 0,3953 \\
\hline 150 & & 0,2011 & 0,1976 & 0,3685 & 0,6443 & & 0,0723 & 0,3687 & 0,3685 & 0,6443 & & 0,0548 & 0,5025 & 0,3685 & 0,6443 \\
\hline 200 & & 0,2648 & 0,3021 & 0,4205 & 0,8237 & & 0,0785 & 0,4902 & 0,4205 & 0,8237 & & 0,0547 & 0,637 & 0,4205 & 0,8237 \\
\hline 250 & Log-Rank & 0,3209 & 0,4507 & 0,4782 & 0,9239 & Tarone e Ware & 0,0868 & 0,6183 & 0,4783 & 0,9239 & Peto e Peto & 0,0593 & 0,7523 & 0,4782 & 0,9239 \\
\hline 300 & & 0,3748 & 0,5967 & 0,5186 & 0,9687 & $\gamma=0,5$ & 0,0932 & 0,7239 & 0,5186 & 0,9687 & & 0,0557 & 0,8298 & 0,5186 & 0,9687 \\
\hline 350 & & 0,4307 & 0,7379 & 0,5852 & 0,9862 & & 0,1002 & 0,8131 & 0,5852 & 0,9863 & & 0,0569 & 0,894 & 0,5852 & 0,986 \\
\hline 400 & & 0,4777 & 0,8512 & 0,6161 & 0,9941 & & 0,1073 & 0,8795 & 0,6163 & 0,9941 & & 0,0621 & 0,9334 & 0,6163 & 0,9941 \\
\hline 450 & & 0,5439 & 0,9262 & 0,6559 & 0,9946 & & 0,1181 & 0,9277 & 0,6559 & 0,9946 & & 0,0591 & 0,9604 & 0,6559 & 0,9946 \\
\hline 500 & & 0,5912 & 0,9629 & 0,6952 & 0,9987 & & 0,1217 & 0,9544 & 0,6952 & 0,9987 & & 0,0576 & 0,9715 & 0,6952 & 0,9987 \\
\hline 60 & & 0,055 & 0,2286 & 0,2611 & 0,2018 & & 0,0575 & 0,1731 & 0,2611 & 0,2018 & & 0,0535 & 0,2138 & 0,2611 & 0,2018 \\
\hline 80 & & 0,0577 & 0,2905 & 0,2807 & 0,3039 & & 0,059 & 0,2208 & 0,2807 & 0,3039 & & 0,0563 & 0,2772 & 0,2807 & 0,3039 \\
\hline 100 & & 0,0529 & 0,3655 & 0,3054 & 0,3953 & & 0,0541 & 0,2755 & 0,3054 & 0,3953 & & 0,0513 & 0,3455 & 0,3054 & 0,3953 \\
\hline 150 & & 0,0585 & 0,5292 & 0,3685 & 0,6443 & & 0,0547 & 0,3993 & 0,3685 & 0,6443 & & 0,0549 & 0,5034 & 0,3685 & 0,6443 \\
\hline 200 & & 0,0586 & 0,6615 & 0,4205 & 0,8237 & & 0,0573 & 0,5143 & 0,4205 & 0,8237 & & 0,0547 & 0,6381 & 0,4205 & 0,8237 \\
\hline 250 & Gehan & 0,0655 & 0,7747 & 0,478 & 0,9239 & Fleming e & 0,0587 & 0,6306 & 0,4782 & 0,9239 & Peto e Peto & 0,0593 & 0,7527 & 0,478 & 0,9239 \\
\hline 300 & & 0,0627 & 0,8476 & 0,5186 & 0,9687 & Harrington & 0,0566 & 0,7182 & 0,5186 & 0,9687 & Modificado & 0,0557 & 0,8298 & 0,5186 & 0,9687 \\
\hline 350 & & 0,0664 & 0,9074 & 0,5852 & 0,986 & $\gamma=0,5 \mathrm{e}$ & 0,0579 & 0,7963 & 0,5852 & 0,9863 & & 0,0569 & 0,8941 & 0,5852 & 0,9861 \\
\hline 400 & & 0,0692 & 0,9442 & 0,616 & 0,9941 & $\rho=2$ & 0,0566 & 0,8543 & 0,6163 & 0,9941 & & 0,0622 & 0,9338 & 0,616 & 0,9941 \\
\hline 450 & & 0,0681 & 0,9682 & 0,6559 & 0,9946 & & 0,0592 & 0,8979 & 0,6559 & 0,9946 & & 0,0591 & 0,9604 & 0,6559 & 0,9946 \\
\hline 500 & & 0,0694 & 0,9765 & 0,6952 & 0,9987 & & 0,0586 & 0,9283 & 0,6952 & 0,9987 & & 0,0578 & 0,9715 & 0,6952 & 0,9987 \\
\hline
\end{tabular}

Tabela 4.14: Tabela da função poder do testes para 2 cruzamentos, 3 populações e sem censura.

No terceiro experimento, observamos que o teste Qui-Quadrado têm desempenho melhor frente aos demais testes. Além disso, a estatística CVM é mais poderosa que as estatísticas MLR e LRP, para qualquer função de ponderação. Notamos também que LRP não aproxima 1 para quaisquer funções de ponderação, quando aumentamos o tamanho da amostra. 
Peso: Log-rank

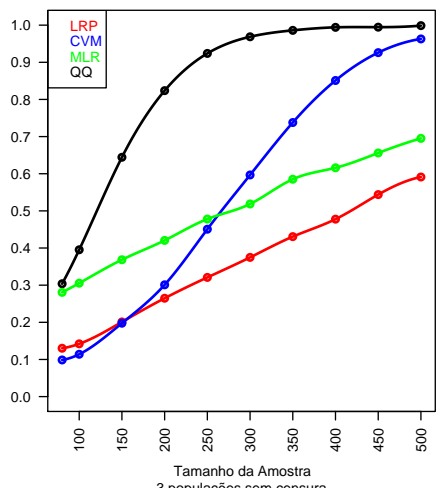

Peso: Fleming e Harrington, Gamma=0.5,Rho=2

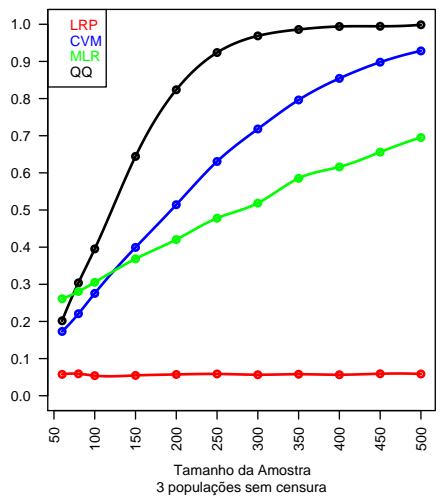

Peso: Gehan

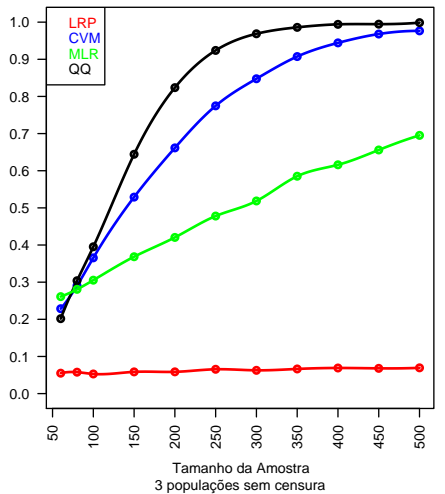

Peso: Peto e Peto

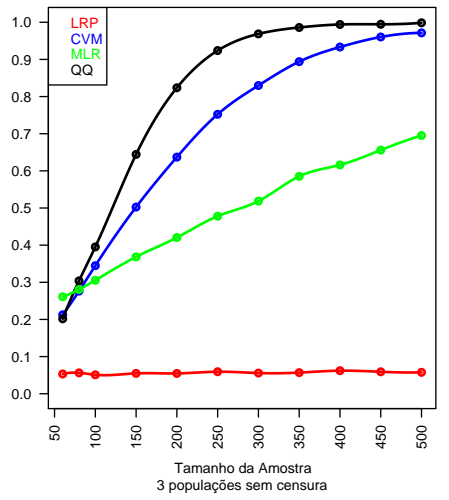

Peso: Tarone e Ware, Gamma $=0.5$

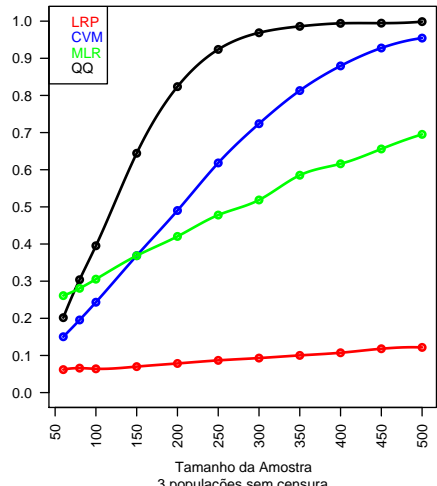

Peso: Peto e Peto Modificado

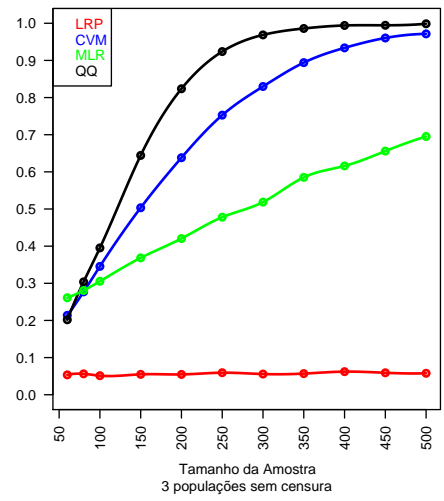

Figura 4.15: Gráfico do poder do testes para 2 cruzamentos, 3 populações e sem censura.

\begin{tabular}{|c|c|c|c|c|c|c|c|c|c|c|c|c|c|c|}
\hline TA & Peso & LRP & CVM & MLR & Peso & LRP & CVM & MLR & Peso & LRP & CVM & MLR & Cens1 \% & Cens $2 \%$ \\
\hline 60 & & 0,2176 & 0,2588 & 0,2029 & & 0,2252 & 0,2746 & 0,2029 & & 0,2287 & 0,2804 & 0,2029 & & \\
\hline 80 & & 0,2588 & 0,3227 & 0,2553 & & 0,272 & 0,3448 & 0,2553 & & 0,2759 & 0,3524 & 0,2553 & & \\
\hline 100 & & 0,329 & 0,4006 & 0,3336 & & 0,3441 & 0,4246 & 0,3336 & & 0,3487 & 0,4381 & 0,3336 & & \\
\hline 150 & & 0,4412 & 0,5502 & 0,4655 & & 0,4584 & 0,5854 & 0,4655 & & 0,4676 & 0,5995 & 0,4655 & & \\
\hline 200 & & 0,5413 & 0,6633 & 0,5937 & & 0,5681 & 0,7001 & 0,5937 & & 0,5792 & 0,7183 & 0,5937 & & \\
\hline 250 & Log-Rank & 0,6291 & 0,7558 & 0,7022 & Tarone e Ware & 0,6559 & 0,7944 & 0,7022 & Peto e Peto & 0,6711 & 0,8112 & 0,7022 & 54,86 & 58,23 \\
\hline 300 & & 0,6971 & 0,8242 & 0,7829 & $\gamma=0,5$ & 0,7233 & 0,8576 & 0,7829 & & 0,7386 & 0,8719 & 0,7829 & & \\
\hline 350 & & 0,7477 & 0,8754 & 0,8489 & & 0,7761 & 0,9043 & 0,8489 & & 0,7921 & 0,9164 & 0,8489 & & \\
\hline 400 & & 0,7854 & 0,9143 & 0,9321 & & 0,8171 & 0,9403 & 0,9321 & & 0,8342 & 0,9502 & 0,9322 & & \\
\hline 450 & & 0,8154 & 0,9447 & 0,9298 & & 0,8489 & 0,9658 & 0,9298 & & 0,8676 & 0,9708 & 0,9298 & & \\
\hline 500 & & 0,8414 & 0,9615 & 0,9577 & & 0,8728 & 0,9783 & 0,9577 & & 0,8913 & 0,9833 & 0,9577 & & \\
\hline 60 & & 0,2353 & 0,2853 & 0,2029 & & 0,2119 & 0,2538 & 0,2029 & & 0,2288 & 0,2805 & 0,2029 & & \\
\hline 80 & & 0,2837 & 0,3589 & 0,2553 & & 0,2539 & 0,3107 & 0,2553 & & 0,2764 & 0,3525 & 0,2553 & & \\
\hline 100 & & 0,3563 & 0,4459 & 0,3336 & & 0,3231 & 0,3878 & 0,3336 & & 0,3487 & 0,4381 & 0,3336 & & \\
\hline 150 & & 0,4774 & 0,6094 & 0,4655 & & 0,4295 & 0,5345 & 0,4655 & & 0,4675 & 0,5998 & 0,4655 & & \\
\hline 200 & & 0,5907 & 0,7293 & 0,5937 & & 0,5267 & 0,6503 & 0,5937 & & 0,5793 & 0,7187 & 0,5937 & & \\
\hline 250 & Gehan & 0,6824 & 0,8182 & 0,7022 & Fleming e & 0,6103 & 0,7442 & 0,7024 & Peto e Peto & 0,6711 & 0,8113 & 0,7023 & 54,86 & 58,23 \\
\hline 300 & & 0,7509 & 0,8794 & 0,7829 & Harrington & 0,6781 & 0,8123 & 0,7829 & Modificado & 0,7386 & 0,8721 & 0,7829 & & \\
\hline 350 & & 0,8016 & 0,9241 & 0,8489 & $\gamma=0,5 \mathrm{e}$ & 0,7324 & 0,8632 & 0,8489 & & 0,7921 & 0,9165 & 0,8489 & & \\
\hline 400 & & 0,8445 & 0,9536 & 0,9234 & $\rho=2$ & 0,7726 & 0,9061 & 0,9431 & & 0,8342 & 0,9504 & 0,9431 & & \\
\hline 450 & & 0,8774 & 0,9732 & 0,9298 & & 0,8008 & 0,9399 & 0,9298 & & 0,8677 & 0,9708 & 0,9298 & & \\
\hline 500 & & 0,9025 & 0,9846 & 0,9577 & & 0,8293 & 0,9573 & 0,9577 & & 0,8913 & 0,9833 & 0,9577 & & \\
\hline
\end{tabular}

Tabela 4.15: Tabela da função poder do testes para 2 cruzamentos, 3 populações e com censura.

No último experimento, observamos que o teste de CVM têm desempenho melhor frente aos demais para qualquer função de ponderação. Notamos também que o teste de LRP detecta diferenças nas funções de intensidade quando censuramos os dados. Resumindo, temos que LRP detecta diferenças nas funções de intensidade apenas no caso de censura dos dados. Já MLR têm maior poder apenas no caso de 2 populações e sem censura, já para 3 populações QQ têm maior poder, por fim, nos demais casos CVM têm poder maior. 
Peso: Log-rank

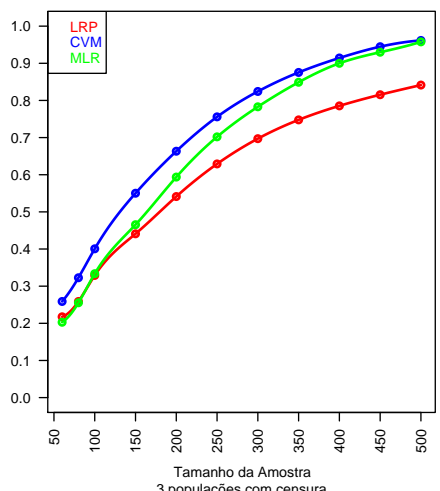

Tamanho da Amostra
populaçōes com censura

Peso: Fleming e Harrington, Gamma $=0.5$, Rho $=2$

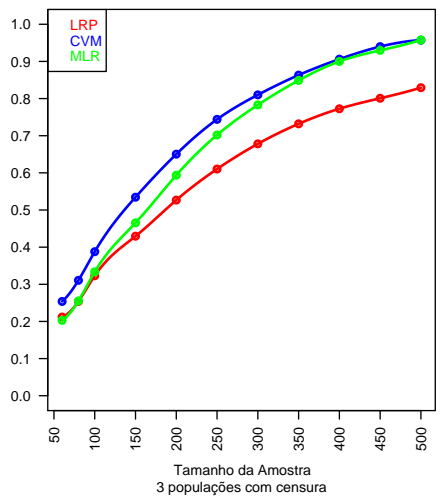

Peso: Gehan

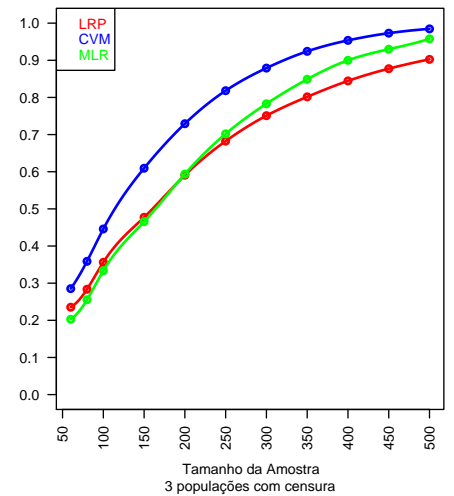

Peso: Peto e Peto

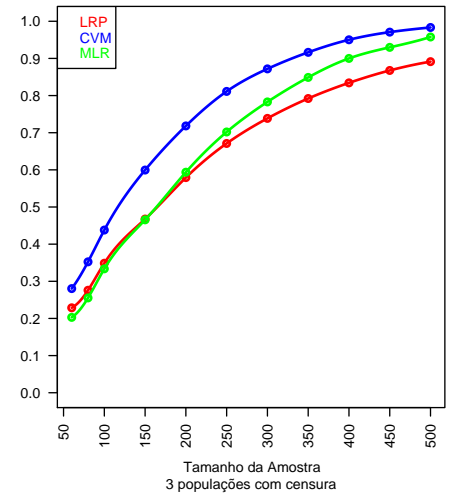

Peso: Tarone e Ware, Gamma $=0.5$

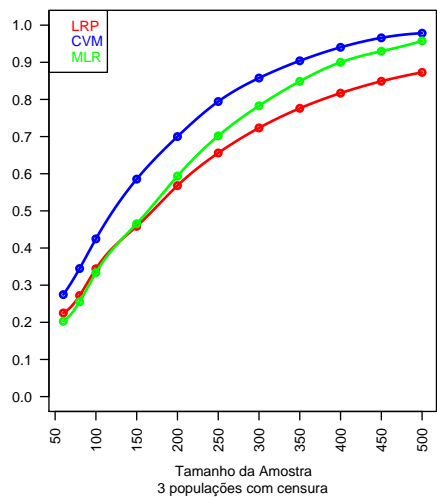

Peso: Peto e Peto Modificado

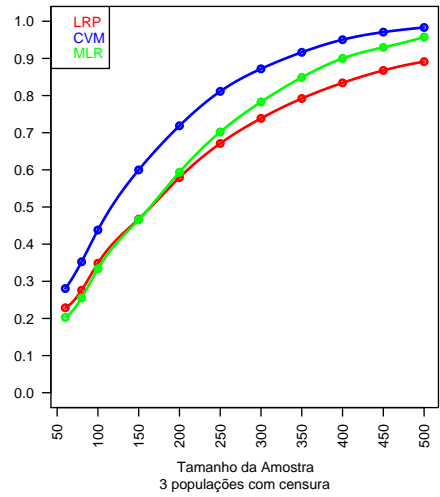

Figura 4.16: Gráfico do poder do testes para 2 cruzamentos, 3 populações e com censura.

\subsection{Espaços Amostrais Infinitos Enumeráveis}

Na seção anterior estudamos o comportamento dos testes para espaços amostrais finitos. Aqui, avaliamos o comportamento dos testes CVM e LRP em diversas situações para espaços amostrais infinitos enumeráveis. No primeiro experimento, avaliamos os testes para riscos proporcionais, em seguida, avaliamos para riscos não proporcionais (cruzamento das funções de intensidade).

\subsubsection{Simulação para riscos proporcionais}

Nesta seção, avaliamos o comportamento das estatísticas LRP e CVM em dados discretos na ausência e presença de censura e funções intensidade proporcionais. Aqui, utilizamos a distribuição Poisson para modelar as populações. No primeiro experimento desta seção, sob a hipótese nula $H_{0}$, analisamos o efeito do tamanho da amostra e do número de populações. Os números de tamanhos de amostra escolhidos para o primeiro experimento são de 50, 100, 150, 200, 250 e 300, já o número de populações são 2,3 , . . e 8 . O parâmetro usado para a distribuição Poisson é $\lambda=100$ e simulamos em situações sem censura e censura à direita em 90,100, 105 e 110. No segundo experimento, avaliamos o poder do teste quando a hipótese alternativa é fixada e com incrementos de $\delta$ adicionada a esta hipótese. A seguir, apresentamos o algoritmo para a simulação sob a hipótese nula:

1. Fixamos um nível de significância $\alpha=0,05$; 
2. Geramos populações de tamanho TA com distribuição Poisson com parâmetro $(\lambda)$ dado que a hipótese nula é verdadeira;

3. Usamos o processo de ponderação $\log -\operatorname{rank} u\left(n^{\star},.\right)=1$;

4. Realizamos o teste de hipótese usando valor crítico ao nível de significância $\alpha$;

5. Verificamos se cometeu o erro do tipo I, isto é, se a hipótese nula foi rejeitada;

$$
y_{m}= \begin{cases}1, & \text { se } H_{0} \text { é rejeitada ao nível de significância } \alpha \\ 0, & \text { caso contrário }\end{cases}
$$

6. Repetimos as etapas 2 a $4 m=10000$ vezes;

7. Estimamos a probabilidade de cometer o erro do tipo I da seguinte forma: $\hat{\alpha}=\frac{1}{m} \sum_{k=1}^{m} y_{k}$

\begin{tabular}{|c|c|c|c|c|c|c|c|c|c|c|c|c|}
\hline \multicolumn{13}{|c|}{ Sem Censura } \\
\hline TA & npop $^{3}$ & CVM & LRP & npop & CVM & LRP & npop & CVM & LRP & npop & CVM & LRP \\
\hline 50 & & 0,0493 & 0,0564 & & 0,0541 & 0,0604 & & 0,0478 & 0,0569 & & 0,0521 & 0,0529 \\
\hline 100 & & 0,0492 & 0,0531 & & 0,0493 & 0,0578 & & 0,0495 & 0,0516 & & 0,0517 & 0,0496 \\
\hline 150 & 2 & 0,054 & 0,0549 & 3 & 0,049 & 0,0479 & 4 & 0,0526 & 0,0501 & 5 & 0,0514 & 0,0525 \\
\hline 200 & & 0,0478 & 0,0493 & & 0,0475 & 0,0516 & & 0,0536 & 0,0527 & & 0,0523 & 0,0472 \\
\hline 250 & & 0,051 & 0,0524 & & 0,0501 & 0,0534 & & 0,0526 & 0,0528 & & 0,0551 & 0,0517 \\
\hline 300 & & 0,0493 & 0,0528 & & 0,0509 & 0,0524 & & 0,0534 & 0,0523 & & 0,0504 & 0,0489 \\
\hline $\mathbf{T A}$ & npop & CVM & LRP & npop & CVM & LRP & npop & CVM & LRP & & & \\
\hline 50 & & 0,0556 & 0,0528 & & 0,0597 & 0,0571 & & 0,0706 & 0,072 & & & \\
\hline 100 & & 0,0585 & 0,0527 & & 0,0551 & 0,056 & & 0,0652 & 0,0602 & & & \\
\hline 150 & 6 & 0,0541 & 0,0504 & 7 & 0,0553 & 0,0489 & 8 & 0,0599 & 0,0593 & & & \\
\hline 200 & & 0,0521 & 0,0501 & & 0,0583 & 0,0537 & & 0,0595 & 0,0544 & & & \\
\hline 250 & & 0,0575 & 0,0524 & & 0,0543 & 0,0509 & & 0,0549 & 0,0563 & & & \\
\hline 300 & & 0,0544 & 0,0499 & & 0,0509 & 0,0445 & & 0,0545 & 0,0541 & & & \\
\hline
\end{tabular}

Tabela 4.16: Avaliação do Erro do Tipo I para riscos proporcionais na ausência de censura.

Nesta primeira situação, estamos na ausência de censura e pelos resultados obtidos na tabela (4.16) notamos que a probabilidade de cometer o erro tipo I para as duas estatísticas estão muito próximas da probabilidade nominal $(\alpha)$. Note que, para $\mathrm{CVM}^{2}$ temos uma instabilidade na convergência para tamanhos de amostras pequenos quando aumentamos o número de populações. Este mesmo fenômeno também ocorre para $\mathrm{LRP}^{3}$.

\footnotetext{
${ }^{3}$ Número de populações

${ }^{2}$ Cramér-von Mises

${ }^{3}$ Log-rank Ponderado
} 
2 populações

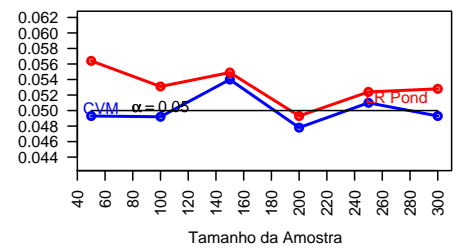

5 populações

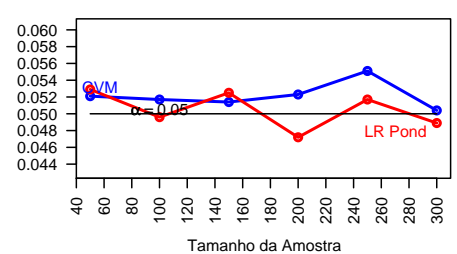

8 populações

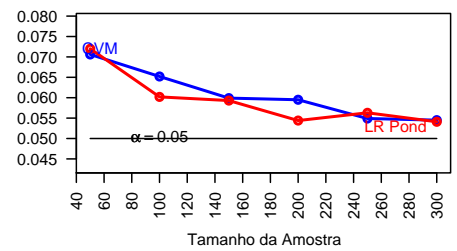

3 populações

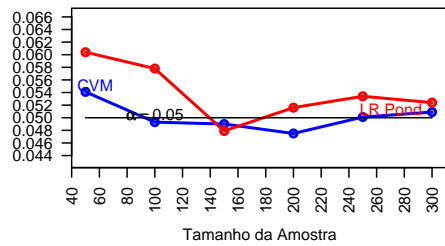

6 populações

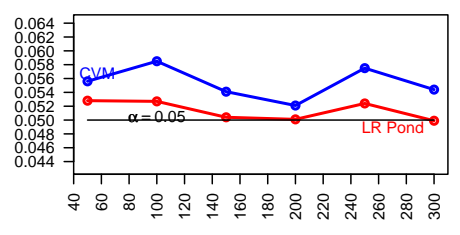

4 populações

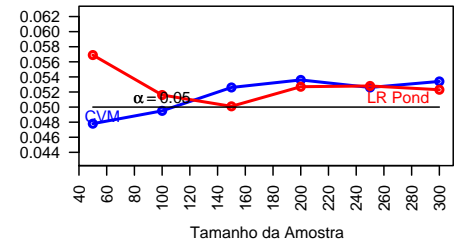

7 populações

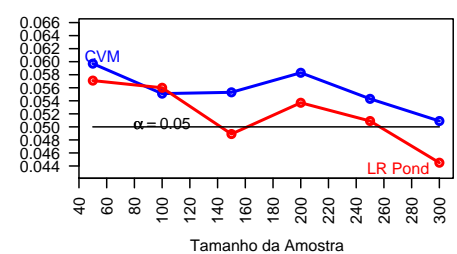

Figura 4.17: Gráfico da simulação sob a hipótese nula $H_{0}$ (sem censura).

\begin{tabular}{|c|c|c|c|c|c|c|c|c|c|c|c|c|}
\hline \multicolumn{13}{|c|}{ Censura 90 à direita } \\
\hline TA & npop & CVM & LRP & npop & CVM & LRP & npop & CVM & LRP & npop & CVM & LRP \\
\hline 50 & & 0,0491 & 0,0477 & & 0,0416 & 0,0567 & & 0,0285 & 0,0711 & & 0,0316 & 0,0787 \\
\hline 100 & & 0,0483 & 0,0484 & & 0,0447 & 0,0502 & & 0,038 & 0,0578 & & 0,0372 & 0,0663 \\
\hline 150 & 2 & 0,048 & 0,0485 & 3 & 0,0465 & 0,0518 & 4 & 0,0457 & 0,0531 & 5 & 0,0499 & 0,057 \\
\hline 200 & & 0,0474 & 0,0482 & & 0,0459 & 0,0456 & & 0,043 & 0,0552 & & 0,0424 & 0,0557 \\
\hline 250 & & 0,0516 & 0,0532 & & 0,0474 & 0,0501 & & 0,0441 & 0,0506 & & 0,0454 & 0,0553 \\
\hline 300 & & 0,0481 & 0,048 & & 0,0476 & 0,0492 & & 0,0429 & 0,0532 & & 0,0412 & 0,0521 \\
\hline TA & npop & CVM & LRP & npop & CVM & LRP & npop & CVM & LRP & & & \\
\hline 50 & & 0,029 & 0,0861 & & 0,0324 & 0,0957 & & 0,0326 & 0,1051 & & & \\
\hline 100 & & 0,0369 & 0,0644 & & 0,0385 & 0,0682 & & 0,0387 & 0,072 & & & \\
\hline 150 & 6 & 0,0458 & 0,0554 & 7 & 0,0446 & 0,0626 & 8 & 0,0446 & 0,0575 & & & \\
\hline 200 & & 0,0401 & 0,056 & & 0,0439 & 0,0587 & & 0,0451 & 0,0635 & & & \\
\hline 250 & & 0,0439 & 0,0584 & & 0,0447 & 0,0576 & & 0,0425 & 0,0569 & & & \\
\hline 300 & & 0,045 & 0,0547 & & 0,0466 & 0,0618 & & 0,0451 & 0,0591 & & & \\
\hline
\end{tabular}

Tabela 4.17: Avaliação do Erro do Tipo I para riscos proporcionais com Censura 90 à direita.

Para os resultados obtidos com censura 90 à direita (ver tabela (4.17)) notamos que os testes convergem para a probabilidade nominal $(\alpha)$. No entanto, quando aumentamos o número de populações e temos censura severa dos dados a convergência é obtida quando aumentamos bastante o tamanho de amostra. Nesta situação os testes têm comportamento inverso, ou seja, quando aumentamos o número de populações LRP 4 têm proporções altas enquanto $\mathrm{CVM}^{5}$ baixas, e ambos convergindo para tamanhos de amostra grandes.

\footnotetext{
${ }^{4}$ Log-rank Ponderado

${ }^{5}$ Cramér-von Mises
} 
2 populações

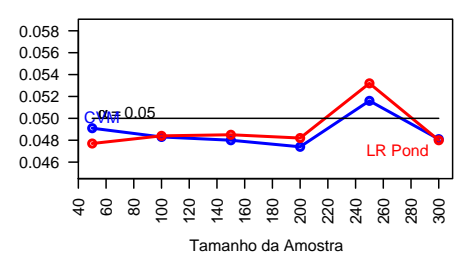

5 populações

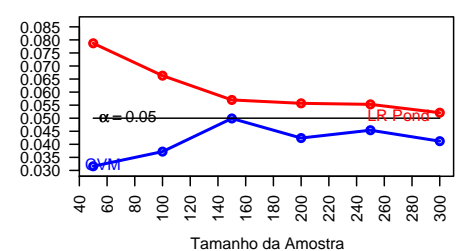

8 populações

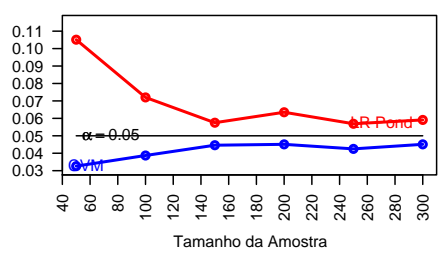

3 populações

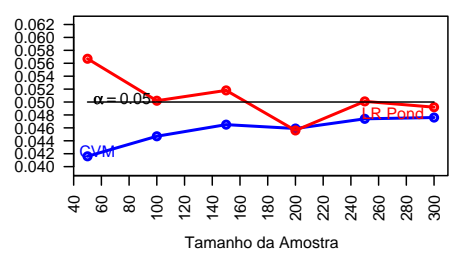

6 populações

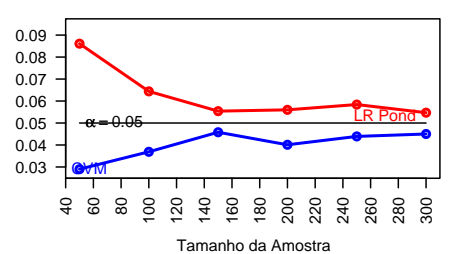

4 populações

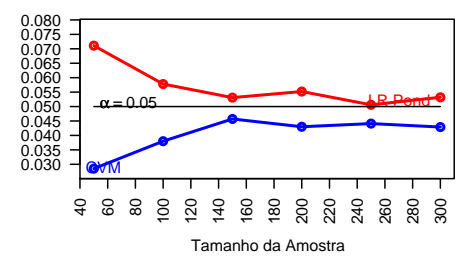

7 populações

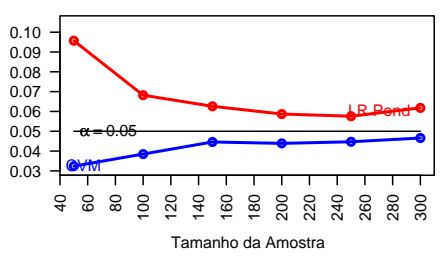

Figura 4.18: Gráfico da simulação sob a hipótese nula $H_{0}$ (censura 90).

\begin{tabular}{|c|c|c|c|c|c|c|c|c|c|c|c|c|}
\hline \multicolumn{13}{|c|}{ Censura 100 à direita } \\
\hline TA & npop & CVM & LRP & npop & CVM & LRP & npop & CVM & LRP & npop & CVM & LRP \\
\hline 50 & & 0,0506 & 0,0513 & & 0,052 & 0,0512 & & 0,0457 & 0,0588 & & 0,0481 & 0,0606 \\
\hline 100 & & 0,0534 & 0,0507 & & 0,0524 & 0,0532 & & 0,0454 & 0,0538 & & 0,0497 & 0,0576 \\
\hline 150 & 2 & 0,0526 & 0,0504 & 3 & 0,051 & 0,0519 & 4 & 0,0497 & 0,0558 & 5 & 0,0502 & 0,0561 \\
\hline 200 & & 0,0504 & 0,0519 & & 0,049 & 0,0491 & & 0,0482 & 0,0525 & & 0,0506 & 0,0526 \\
\hline 250 & & 0,0475 & 0,0487 & & 0,0518 & 0,0501 & & 0,0504 & 0,0525 & & 0,0534 & 0,0542 \\
\hline 300 & & 0,0517 & 0,0547 & & 0,0503 & 0,0498 & & 0,0482 & 0,0499 & & 0,0493 & 0,0526 \\
\hline TA & npop & CVM & LRP & npop & CVM & LRP & npop & CVM & LRP & & & \\
\hline 50 & & 0,0457 & 0,0573 & & 0,0517 & 0,064 & & 0,0535 & 0,0682 & & & \\
\hline 100 & & 0,0477 & 0,0526 & & 0,0513 & 0,0615 & & 0,0512 & 0,0595 & & & \\
\hline 150 & 6 & 0,0449 & 0,0518 & 7 & 0,0466 & 0,0548 & 8 & 0,0535 & 0,0581 & & & \\
\hline 200 & & 0,0517 & 0,056 & & 0,0494 & 0,0486 & & 0,0529 & 0,0558 & & & \\
\hline 250 & & 0,0497 & 0,051 & & 0,0474 & 0,0491 & & 0,0456 & 0,051 & & & \\
\hline 300 & & 0,051 & 0,0547 & & 0,0496 & 0,0503 & & 0,0505 & 0,0539 & & & \\
\hline
\end{tabular}

Tabela 4.18: Avaliação do Erro do Tipo I para riscos proporcionais com Censura 100 à direita.

Para os resultados obtidos com censura 100 à direita (ver tabela (4.18)) notamos que as duas estatísticas convergem para a probabilidade nominal $(\alpha)$. Neste caso, com censura moderada, notamos um comportamento melhor da estatística CVM que da estatística LRP. 
2 populações

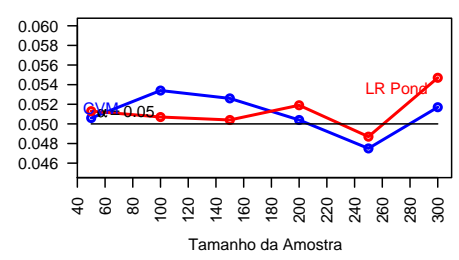

5 populações

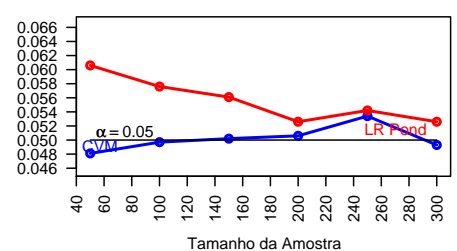

8 populações

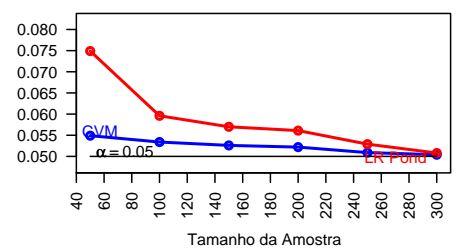

3 populações

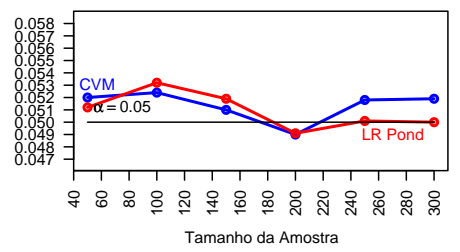

6 populações

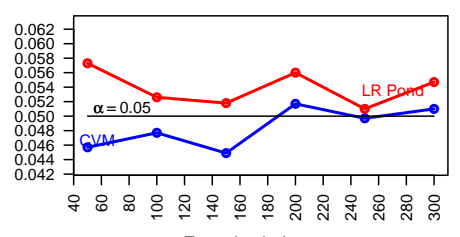

4 populações

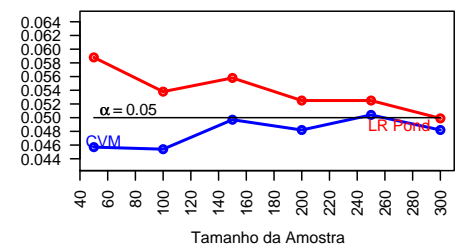

7 populações

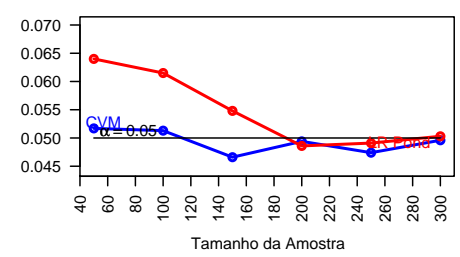

Figura 4.19: Gráfico da simulação sob a hipótese nula $H_{0}$ (censura 100).

\begin{tabular}{|c|c|c|c|c|c|c|c|c|c|c|c|c|}
\hline \multicolumn{13}{|c|}{ Censura 105 à direita } \\
\hline TA & npop & CVM & LRP & npop & CVM & LRP & npop & CVM & LRP & npop & CVM & LRP \\
\hline 50 & & 0,0515 & 0,0505 & & 0,047 & 0,049 & & 0,0519 & 0,057 & & 0,0526 & 0,0588 \\
\hline 100 & & 0,0505 & 0,0481 & & 0,0505 & 0,0497 & & 0,0534 & 0,0567 & & 0,0508 & 0,0499 \\
\hline 150 & 2 & 0,0482 & 0,0497 & 3 & 0,0516 & 0,05 & 4 & 0,0485 & 0,0533 & 5 & 0,0517 & 0,0519 \\
\hline 200 & & 0,0532 & 0,0519 & & 0,0482 & 0,0482 & & 0,0478 & 0,0514 & & 0,0466 & 0,0489 \\
\hline 250 & & 0,0509 & 0,051 & & 0,0506 & 0,0479 & & 0,0502 & 0,0484 & & 0,0512 & 0,0514 \\
\hline 300 & & 0,0527 & 0,0533 & & 0,0474 & 0,0461 & & 0,0513 & 0,0501 & & 0,0453 & 0,0487 \\
\hline TA & npop & CVM & LRP & npop & CVM & LRP & npop & CVM & LRP & & & \\
\hline 50 & & 0,0535 & 0,0578 & & 0,061 & 0,0645 & & 0,0626 & 0,0708 & & & \\
\hline 100 & & 0,0532 & 0,0538 & & 0,0546 & 0,0551 & & 0,0534 & 0,0583 & & & \\
\hline 150 & 6 & 0,0488 & 0,0558 & 7 & 0,0543 & 0,0541 & 8 & 0,0581 & 0,059 & & & \\
\hline 200 & & 0,0516 & 0,0497 & & 0,052 & 0,0533 & & 0,0527 & 0,0554 & & & \\
\hline 250 & & 0,0534 & 0,0499 & & 0,0501 & 0,0509 & & 0,0518 & 0,0551 & & & \\
\hline 300 & & 0,0529 & 0,0539 & & 0,0494 & 0,0498 & & 0,051 & 0,056 & & & \\
\hline
\end{tabular}

Tabela 4.19: Avaliação do Erro do Tipo I para riscos proporcionais com Censura 105 à direita.

Para os resultados obtidos com censura 105 à direita (ver tabela (4.19)) notamos que as duas estatísticas aproximam a probabilidade nominal $(\alpha)$ de forma similar. 
2 populações

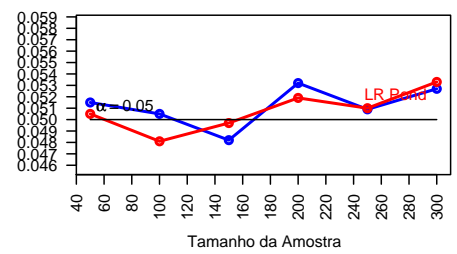

5 populações

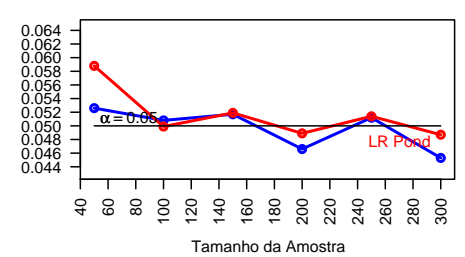

8 populações

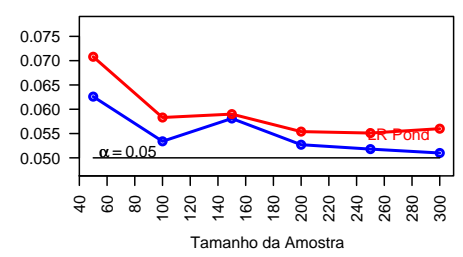

3 populações

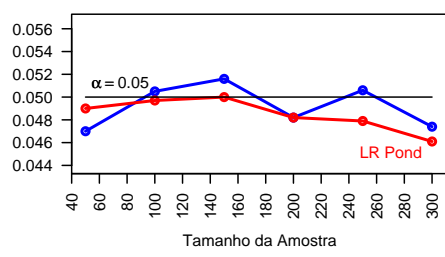

6 populações

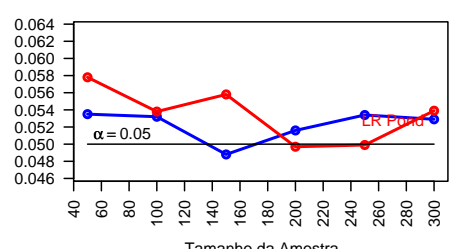

4 populações

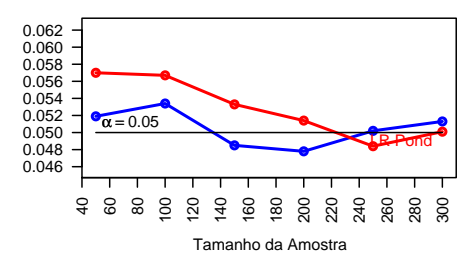

7 populações

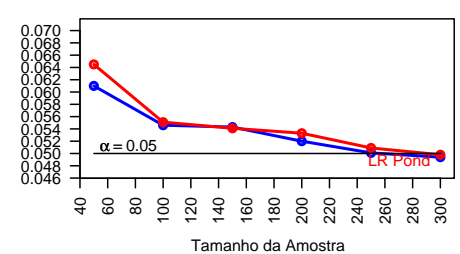

Figura 4.20: Gráfico da simulação sob a hipótese nula $H_{0}$ (censura 105).

\begin{tabular}{|c|c|c|c|c|c|c|c|c|c|c|c|c|}
\hline \multicolumn{13}{|c|}{ Censura 110 à direita } \\
\hline TA & npop & CVM & LRP & npop & CVM & LRP & npop & CVM & LRP & npop & CVM & LRP \\
\hline 50 & & 0,0556 & 0,0537 & & 0,0562 & 0,0523 & & 0,0562 & 0,0523 & & 0,0554 & 0,0552 \\
\hline 100 & & 0,0508 & 0,0506 & & 0,0501 & 0,0468 & & 0,0501 & 0,0468 & & 0,0557 & 0,0521 \\
\hline 150 & 2 & 0,0511 & 0,048 & 3 & 0,0501 & 0,0511 & 4 & 0,0501 & 0,0511 & 5 & 0,052 & 0,0479 \\
\hline 200 & & 0,0545 & 0,0515 & & 0,0492 & 0,0528 & & 0,0492 & 0,0528 & & 0,0566 & 0,0549 \\
\hline 250 & & 0,052 & 0,0523 & & 0,0504 & 0,0514 & & 0,0504 & 0,0514 & & 0,0538 & 0,0528 \\
\hline 300 & & 0,0503 & 0,0504 & & 0,0512 & 0,0529 & & 0,0512 & 0,0529 & & 0,047 & 0,0475 \\
\hline TA & npop & CVM & LRP & npop & CVM & LRP & npop & CVM & LRP & & & \\
\hline 50 & & 0,0579 & 0,0545 & & 0,0608 & 0,0664 & & 0,0712 & 0,0743 & & & \\
\hline 100 & & 0,054 & 0,0544 & & 0,0506 & 0,0594 & & 0,0563 & 0,0636 & & & \\
\hline 150 & 6 & 0,0542 & 0,0511 & 7 & 0,0545 & 0,052 & 8 & 0,0589 & 0,0603 & & & \\
\hline 200 & & 0,0525 & 0,0526 & & 0,0494 & 0,049 & & 0,0495 & 0,0543 & & & \\
\hline 250 & & 0,0511 & 0,0497 & & 0,0514 & 0,051 & & 0,0527 & 0,0562 & & & \\
\hline 300 & & 0,051 & 0,0489 & & 0,052 & 0,051 & & 0,0528 & 0,054 & & & \\
\hline
\end{tabular}

Tabela 4.20: Avaliação do Erro do Tipo I para riscos proporcionais com Censura 110 à direita.

Para os resultados obtidos com censura 110 à direita (ver tabela (4.20)) notamos que as duas estatísticas aproximam a probabilidade nominal $(\alpha)$ de forma similar. O resumo dos resultados obtidos é que a probabilidade de cometer o erro tipo I para as duas estatísticas estão muito próximas da probabilidade nominal $(\alpha)$, ou seja, quando fixamos o nível de significância do teste, a probabilidade de cometer um erro do tipo I está sob controle, mesmo para tamanhos amostrais e número de populações variadas. Notamos também que a convergência é comprometida para tamanhos de amostra pequenos, quando aumentamos o número de populações e também para proporções de censura nos dados, porém, em uma visão global, temos um comportamento satisfatório para as duas estatísticas. 


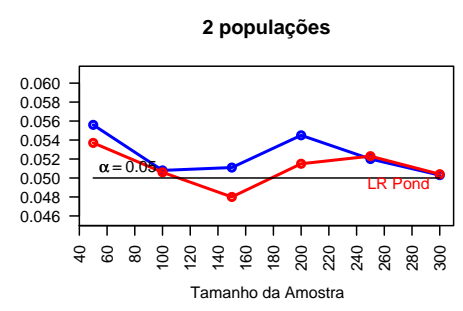

5 populações

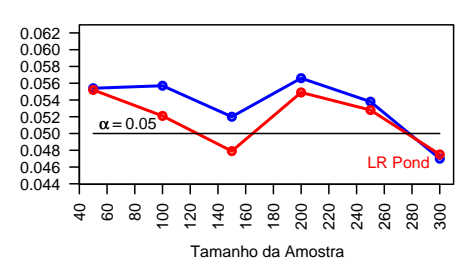

8 populações

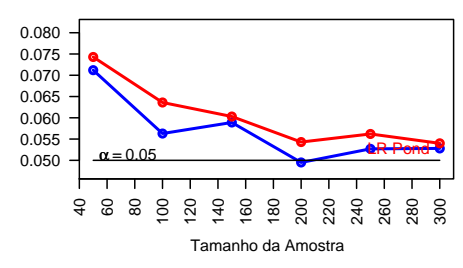

3 populações

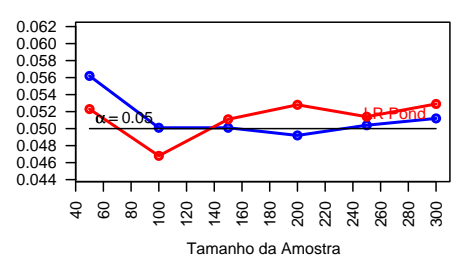

6 populações

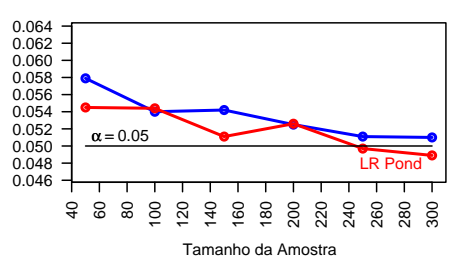

4 populações

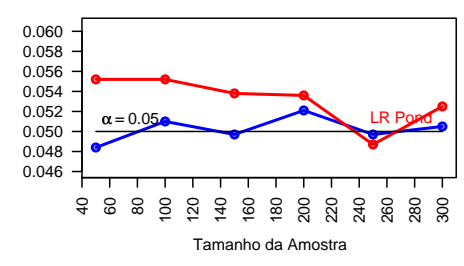

7 populações

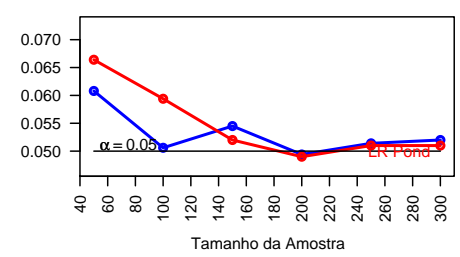

Figura 4.21: Gráfico da simulação sob a hipótese nula $H_{0}$ (censura 110).

O objetivo agora é avaliar a função poder do teste para as estatísticas propostas para dados discretos na ausência e presença de censura, mais especificamente, realizamos um estudo de simulação para avaliar o poder do teste quando a hipótese alternativa é fixada e analisar o efeito do aumento das variações $\delta$ e da censura. Para isto, tomamos duas amostras aleatórias, uma com distribuição Poisson com parâmetro $\lambda_{1}=100$ e outra com a mesma distribuição, porém com parâmetro $\lambda_{2}=\lambda_{1}+\delta$. Assim, o objetivo é avaliar a função poder do teste quando aumentamos o valor de $\delta$ para as estatísticas LRP e CVM. Para o estudo de simulação, os valores de $(\delta)$ escolhidos são $0,2,4,6,8,10,11,12,13,14$ e os de censura à direita 90, 100, 105, 110 e sem censura. Os passos do algoritmo são apresentados à seguir:

1. Fixamos um nível de significância $\alpha=0,05$;

2. Usamos o processo de ponderação log-rank $u\left(n^{\star},.\right)=1$;

3. Fixamos o tamanho da amostra em 100 e definimos duas populações com distribuição Poisson:

- $X^{(1)} \sim P(\lambda)$

- $X^{(2)} \sim P(\lambda+\delta), \operatorname{com} \lambda=100$ dado que a hipótese nula é falsa;

4. Realizamos o teste de hipótese usando valor crítico ao nível de significância $\alpha$;

5. Para estimarmos a função poder tomamos:

$$
y_{m}= \begin{cases}1, & \text { se } H_{0} \text { foi rejeitada ao nível de significância } \alpha \\ 0, & \text { caso contrário }\end{cases}
$$


6. Repetimos as etapas 2 a $4 m=10000$ vezes;

7. Estimamos o poder do teste da seguinte forma:

$$
\text { Poder }=\frac{1}{m} \sum_{k=1}^{m} y_{k}
$$

\begin{tabular}{|c|c|c|c|c|c|c|c|c|c|c|}
\hline \multirow{3}{*}{$\delta$} & \multicolumn{8}{|c|}{ Presença de Censura } & \multicolumn{2}{|c|}{ Ausência de Censura } \\
\hline & \multicolumn{2}{|c|}{90} & \multicolumn{2}{|c|}{100} & \multicolumn{2}{|c|}{105} & \multicolumn{2}{|c|}{110} & \multirow[b]{2}{*}{ LRP } & \multirow[b]{2}{*}{ CVM } \\
\hline & LRP & CVM & LRP & CVM & LRP & CVM & LRP & CVM & & \\
\hline 0 & 0,0477 & 0,0494 & 0,0505 & 0,0524 & 0,0558 & 0,0532 & 0,0544 & 0,0541 & 0,047 & 0,0539 \\
\hline 2 & 0,1272 & 0,1357 & 0,2151 & 0,224 & 0,2391 & 0,2517 & 0,2504 & 0,2664 & 0,2546 & 0,2742 \\
\hline 4 & 0,3487 & 0,3672 & 0,6331 & 0,658 & 0,6987 & 0,7235 & 0,732 & 0,7617 & 0,7409 & 0,7806 \\
\hline 6 & 0,5785 & 0,6166 & 0,9132 & 0,9291 & 0,9521 & 0,9638 & 0,9675 & 0,9753 & 0,9749 & 0,9824 \\
\hline 8 & 0,7655 & 0,8098 & 0,9905 & 0,9931 & 0,9972 & 0,9987 & 0,9983 & 0,9994 & 0,9994 & 0,9998 \\
\hline 10 & 0,8789 & 0,9129 & 0,9995 & 0,9998 & 0,9999 & 0,9999 & 0,9999 & 0,9999 & 1 & 1 \\
\hline 11 & 0,9167 & 0,9503 & 0,9998 & 1 & 1 & 1 & 1 & 1 & 1 & 1 \\
\hline 12 & 0,9381 & 0,9656 & 0,9999 & 1 & 1 & 1 & 1 & 1 & 1 & 1 \\
\hline 13 & 0,9572 & 0,977 & 1 & 1 & 1 & 1 & 1 & 1 & 1 & 1 \\
\hline 14 & 0,9693 & 0,9836 & 1 & 1 & 1 & 1 & 1 & 1 & 1 & 1 \\
\hline
\end{tabular}

Tabela 4.21: Avaliação da função Poder do Teste para Riscos Proporcionais.

Compração entre os Testes

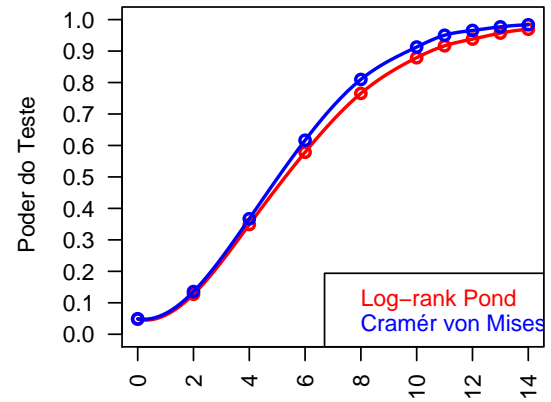

$\stackrel{\delta}{\text { Censura } 90}$

Compração entre os Testes

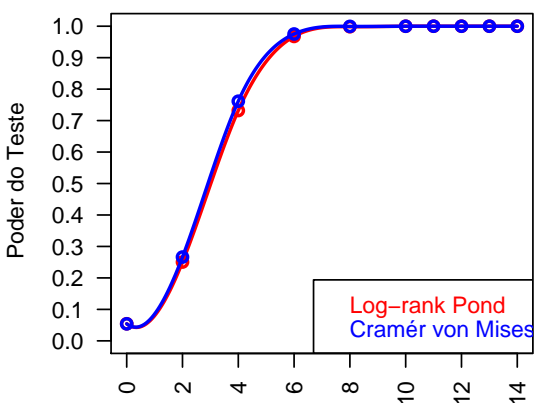

$\stackrel{\delta}{\text { Censura } 110}$
Compração entre os Testes

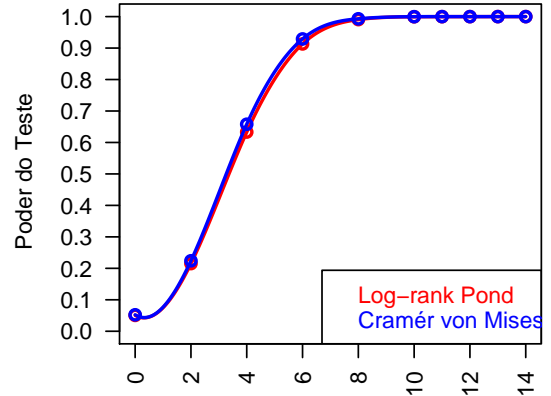

$\stackrel{\delta}{\text { Censura }} 100$

Compração entre os Testes

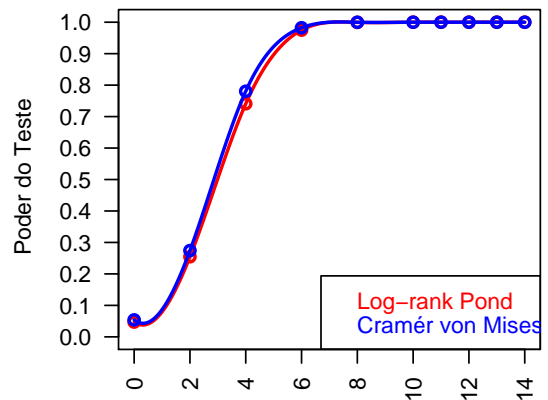

$\stackrel{\delta}{\text { Sem Censura }}$

Figura 4.22: Gráficos da Função Poder $\lambda_{1}=\lambda=100$ e $\lambda_{2}=\lambda+\delta$ 
Na tabela (4.21), temos resumo dos resultados simulados e nela observamos que a taxa de convergência da função poder para os dois testes são similares, sendo um pouco maior para a estatística de Cramérvon Mises quando estamos sob censura severa (censura 90). Notamos também, aumento da função poder quando diminuímos a proporção de censura dos dados.

\subsubsection{Simulação com cruzamento das funções de intensidade}

Os testes apresentados neste trabalho visam comparar as funções de intensidade ou taxas de falha para duas ou mais populações. Todos estes testes foram baseados no "martingale array difference" entre a estimativa acumulada das funções de intensidade nas amostras. Quando estes testes são aplicados a amostras de populações, em que cruzamos as funções de intensidade, estes testes têm poder baixo, porque as diferenças iniciais em favor de um grupo são canceladas por diferenças anteriores em favor do outro tratamento. Nesta seção, vamos comparar as estatísticas propostas neste trabalho com outras estatísticas como a de Renyi (ver em Klein and Moeschberger (1997)[15]), para isto, foi feito um estudo de simulação em dados discretos na ausência de censura para populações com distribuição Poisson e a outra com distribuição Geométrica ${ }^{6}$, mais especificamente, avaliamos o comportamento da função poder em relação aos tamanhos de amostra. A seguir, apresentamos o algoritmo.

1. Fixamos um nível de significância $\alpha=0,05$;

2. Usamos o processo de ponderação log-rank $u\left(n^{\star},.\right)=1$;

3. Geramos populações de tamanho da amostra TA e definimos duas populações com distribuições:

- $X^{(1)} \sim P(\lambda)$ em que $\lambda=1$

- $X^{(2)} \sim \mathrm{G}(p)$ em que $p=0,08$ para o $1^{\circ}$ experimento, $p=0,3$ para o $2^{\circ}$ e $p=0,45$ para o $3^{\circ}$ dado que a hipótese nula é falsa;

4. Realizamos o teste de hipótese usando valor crítico ao nível de significância $\alpha$;

5. Para estimarmos a função poder tomamos:

$$
y_{m}= \begin{cases}1, & \text { se } H_{0} \text { foi rejeitada ao nível de significância } \alpha \\ 0, & \text { caso contrário }\end{cases}
$$

6. Repetimos as etapas 2 a $4 m=10000$ vezes;

7. Estimamos o poder do teste da seguinte forma:

$$
\text { Poder }=\frac{1}{m} \sum_{k=1}^{m} y_{k}
$$




\section{Taxas de Falha}

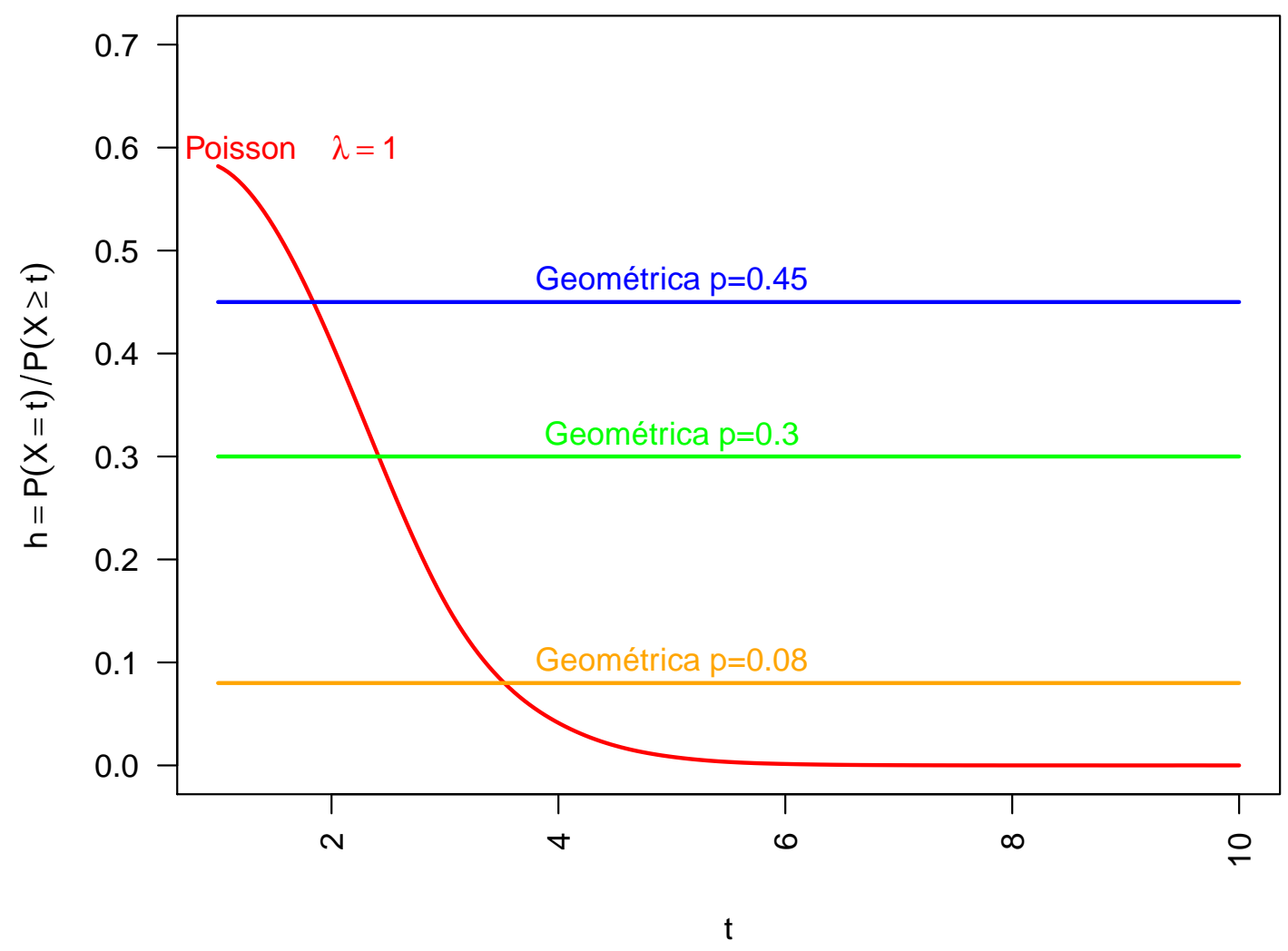

Figura 4.23: Cruzamento das funções de intensidade.

No exemplo (2.1), foi mostrado que a taxa de falha da distribuição Geométrica é dado pelo escalar $p$. Nosso objetivo é avaliar o comportamento dos testes para "cruzamento no início", " no meio" e " no fim" das funções de intensidade. Para isto, tomamos uma distribuição Poisson com parâmetro $\lambda=1$ para a população de controle e outras três populações de tratamentos com distribuição Geométrica como na figura (4.23), com parâmetros $p_{1}=0,45, p_{2}=0,3$ e $p_{3}=0,08$.

Na tabela (4.22), temos um resumo dos resultados simulados e nela observamos que a função poder empírica para a estatística de CVM é melhor em todas as situações em que há cruzamento das funções de intensidade, exceto no caso do cruzamento no fim, em que os dois testes são similares.

\subsubsection{Comparação das funções de ponderação para cruzamento das funções de in- tensidade}

No livro de Klein and Moeschberger (1997)[15] para tratar dados com cruzamentos da função de intensidade é proposto a estatística de Renyi que é análogo a estatística de Kolmogorov-Smirnov para comparar duas amostras na ausência de censura. A construção do teste é baseado no teste estatístico da equação (4.1)

${ }^{6}$ A parametrização da distribuição Geométrica usada está no Apêndice (A.2) 


\begin{tabular}{|c|c|c|c|c|c|c|}
\hline $\begin{array}{l}\text { Poisson } \\
\text { Geométrica } \\
\text { TA }\end{array}$ & \multicolumn{2}{|c|}{$\begin{array}{c}\text { Cruzamento no início } \\
\begin{array}{c}\lambda=1 \\
p=0,45\end{array}\end{array}$} & \multicolumn{2}{|c|}{$\begin{array}{c}\text { Cruzamento no meio } \\
\lambda=1 \\
p=0,3\end{array}$} & \multicolumn{2}{|c|}{$\begin{array}{c}\text { Cruzamento no fim } \\
\lambda=1 \\
p=0,08\end{array}$} \\
\hline 10 & 0,0614 & 0,0933 & 0,1424 & 0,2183 & 0,6042 & 0,6454 \\
\hline 15 & 0,4831 & 0,7821 & 0,15 & 0,2469 & 0,8242 & 0,8379 \\
\hline 30 & 0,6702 & 0,961 & 0,1692 & 0,3842 & 0,9394 & 0,937 \\
\hline 50 & 0,8272 & 0,9982 & 0,2314 & 0,6032 & 0,9911 & 0,993 \\
\hline 100 & 0,9737 & 1 & 0,3132 & 0,9656 & 0,9986 & 0,9997 \\
\hline 150 & 0,9959 & 1 & 0,3701 & 0,9996 & 0,9992 & 1 \\
\hline
\end{tabular}

Tabela 4.22: Função Poder do Teste para cruzamento das funções de intensidade.
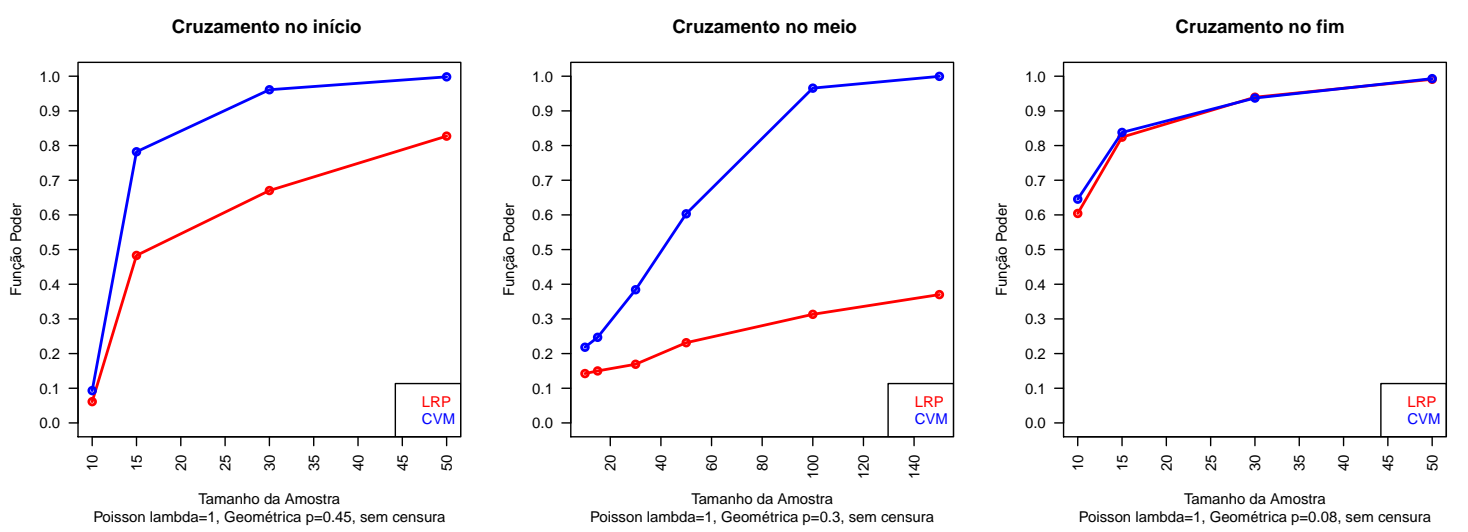

Figura 4.24: Função Poder para cruzamento das funções de intensidade.

para alguma função peso $u\left(n^{\star}, \cdot\right)$.

$$
\begin{aligned}
Z^{q}\left(n^{\star}, i\right) & :=\sum_{\ell=1}^{k} u\left(n^{\star}, \ell\right)\left[\Delta R^{n_{q}}(\ell)-V^{n_{q}}(\ell) \frac{\Delta R^{n^{\star}}(\ell)}{V^{n^{\star}}(\ell)}\right], n^{\star} \in \mathbb{N}^{J}, 1 \leq i \leq k . \\
& \equiv \sqrt{n} L R_{q}\left(n^{\star}, i\right), \quad n^{\star} \in \mathbb{N}^{J}, 1 \leq i \leq k .
\end{aligned}
$$

Para construir o teste, tomamos o caso de dois tratamentos. Suponhamos duas amostras independentes de tamanhos de amostra $n_{1}$ e $n_{2}$. Para a j-ésima amostra, temos que $\Delta R^{n_{j}}(\ell)$ o número de falhas e $V^{n_{j}}(\ell)$ a quantidade sob risco para as categorias $\ell \in \mathcal{K}$. A variância é dada pela equação (4.3).

$$
\sigma^{2}(k):=\sum_{\ell=1}^{k} u^{2}\left(n^{\star}, \ell\right)\left(\frac{V^{n_{1}}(\ell) V^{n_{2}}(\ell)}{V^{n^{\star}}(\ell)}\right)\left(\frac{V^{n^{\star}}(\ell)-\Delta R^{n^{\star}}(\ell)}{V^{n^{\star}}(\ell)-1}\right), n^{\star} \in \mathbb{N}^{J} .
$$

em que $k$ é a maior categoria com $V^{n_{1}}(\ell)>0, V^{n_{2}}(\ell)>0$.

Assim, a estatística de Renyi é dada por

$$
Q=\frac{\sup \left\{\left|Z^{q}\left(n^{\star}, \ell\right)\right|, \quad \ell \leq k\right\}}{\sigma^{2}(k)}
$$

Quando a hipótese nula é verdadeira, então, a distribuição de Q pode ser aproximada pela distribuição do $\sup (|B(x)|, 0<x<1)$, em que $B$ é o movimento Browniano. Os valores críticos de Q são encontrados 
na Tabela C.5 no Apêndice C do livro de Klein and Moeschberger (1997)[15].

Afim de comparar o teste de Cramér-von Mises para dados discretos com outros testes, como o de Logrank ponderado e o de Renyi, usamos o mesmo parâmetro e distribuição para a população de controle no experimento da seção (4.2.2), ou seja, Poisson com parâmetro $\lambda=1$, já para a população de tratamento tomamos ela com distribuição Geométrica e parâmetro $p=0,3$ (cruzamento no meio). Para os experimentos usamos diversas funções de ponderação apresentadas na tabela (3.1) da seção (3.1) na ausência e presença de censura. No primeiro experimento estamos sob a hipótese nula $H_{0}$, para avaliarmos a convergência de todos os testes utilizados. A seguir, apresentamos o algoritmo.

1. Fixamos um nível de significância $\alpha=0,05$;

2. Escolhemos uma das funções de ponderação descrita na tabela (3.1);

3. Geramos populações de tamanho da amostra TA e definimos duas populações com distribuições:

- $X^{(1)} \sim P(\lambda)$ em que $\lambda=1$

- $X^{(2)} \sim \mathrm{G}(p)$ em que $p=0,3$ dado que a hipótese nula é falsa;

4. Realizamos o teste de hipótese usando valor crítico ao nível de significância $\alpha$;

5. Para estimarmos a função poder tomamos: $y_{m}=\left\{\begin{array}{cc}1, & \text { se } H_{0} \text { foi rejeitada ao nível de significância } \alpha \\ 0, & \text { caso contrário }\end{array}\right.$

6. Repetimos as etapas 2 a $4 m=10000$ vezes;

7. Estimamos o poder do teste da seguinte forma: Poder $=\frac{1}{m} \sum_{k=1}^{m} y_{k}$

No primeiro experimento, estamos sob a hipótese nula, e simulamos para duas populações assumindo um número enumerável de valores. Assumimos que $W_{1}$ e $W_{2}$ tem distribuição Poisson com parâmetro $\lambda=1$ na ausência e presença de censura $\left(C_{i} \sim \operatorname{Poi}(\lambda=1)\right)$ com tamanhos de amostra 50, 75, 100, 125 e 200. Observe na tabela (4.23) e na figura (4.25) que os níveis de significância empíricos para as LRP e CVM estão muito próximos do nível de significância nominal 0,05, mas para o teste de Renyi não temos o mesmo comportamento, o nível de significância empírico está abaixo do valor nominal. Assim, para a avaliação da função poder, não vamos considerar a estatística de Renyi.

\begin{tabular}{|c|c|c|c|c|c|c|c|c|c|}
\hline \multirow[t]{2}{*}{ Peso } & \multirow[t]{2}{*}{$\mathbf{T A}$} & \multicolumn{3}{|c|}{ Ausência de Censura } & \multicolumn{3}{|c|}{ Presença de Censura } & \multicolumn{2}{|c|}{$\%$ Censura } \\
\hline & & LRP & CVM & Renyi & LRP & CVM & Renyi & Referência & Controle \\
\hline \multirow[t]{5}{*}{ Log-Rank } & 50 & 0,0521 & 0,0573 & 0,0351 & 0,0569 & 0,0583 & 0,0342 & & \\
\hline & 75 & 0,0494 & 0,0548 & 0,0356 & 0,0537 & 0,0577 & 0,0311 & 45,47 & 45,39 \\
\hline & 100 & 0,049 & 0,0488 & 0,0337 & 0,0476 & 0,046 & 0,0256 & & \\
\hline & 125 & 0,0533 & 0,0519 & 0,037 & 0,0518 & 0,0489 & 0,0284 & & \\
\hline & 200 & 0,0505 & 0,0461 & 0,0283 & 0,0506 & 0,0502 & 0,0275 & & \\
\hline
\end{tabular}

Tabela 4.23: Simulação sob $H_{0}$ para vários tamanhos de amostra e $u\left(n^{\star}, \cdot\right)=1$. 
Resultados sob H_O (Ausência de Censura)

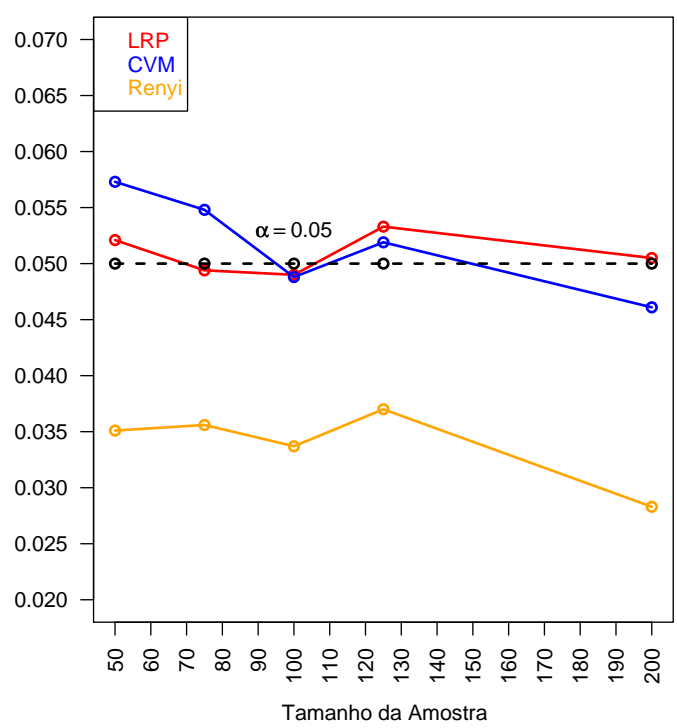

Resultados sob H_O (Presença de Censura)

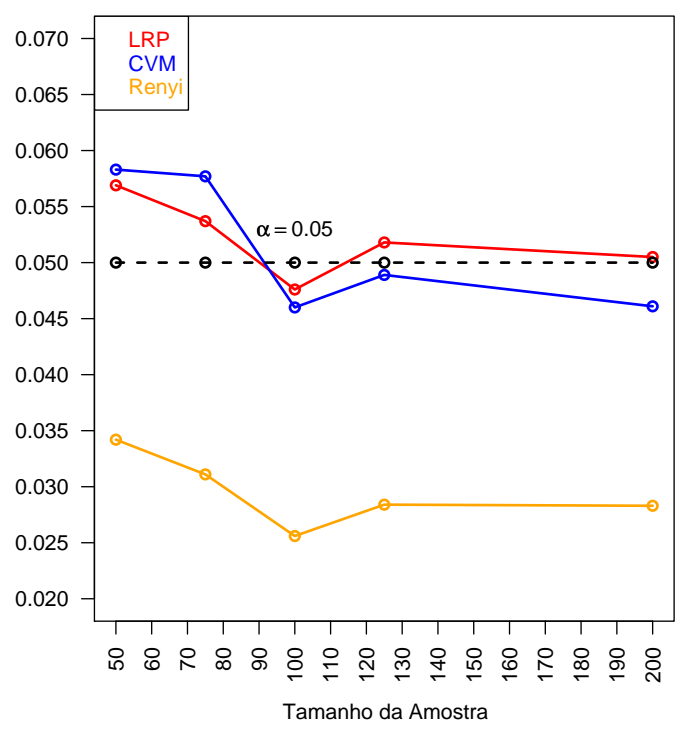

Figura 4.25: Gráfico da Simulação sob $H_{0}$ para vários tamanhos de amostra e $u\left(n^{\star}, \cdot\right)=1$.

A seguir, tomamos o caso mais severo de cruzamento das funções de intensidade ("cruzamento no meio") mostrado na figura (4.23). O cruzamento severo das funções de intensidade tem como consequência cancelamento das diferenças positivas e negativas entre as funções de intensidade estimadas, ocorrendo dificuldade na detecção de diferenças entre os tratamentos. Para o caso em que censuramos os dados, adotamos censura à direita igual a 2 que corresponde a censurarmos 26,66\% para os dados com distribuição Poisson e 42,12\% para os dados com distribuição Geométrica.

Nos experimentos apresentados, geramos para $W^{n_{1}}$ amostras aleatórias com distribuição de Poisson com parâmetro $\lambda=1$, já para $W^{n_{2}}$, geramos amostras aleatórias com distribuição geométrica com parâmetro $p=0,3$. Neste caso, as duas funções de intensidade cruzam entre $\ell=0 \mathrm{e} \ell=1$. Notamos que as variáveis de interesse $W^{n_{1}}$ e $W^{n_{2}}$ tem 36,8\% (40\%) de chance de ser 0 e 63,2\% (60\%) de chance de estar localizado após o ponto de cruzamento $\ell=0$. Notamos que o teste de Cramér-von Mises têm desempenho melhor do que a Log-rank Ponderado, fato ocorrido devido ao cruzamento das funções de intensidade. No entanto, quando os dados são censurados, o teste de Log-rank Ponderado têm desempenho tão bom quanto o teste de Cramér-von Mises, exceto quando usamos a função de ponderação Tarone e Ware $(\gamma=0,5)$, em que Cramér-von Mises tem desempenho bem melhor. Neste experimento foram considerados um tipo de esquema de censura cujo valor é $\ell=2$. Assim, somente o evento localizado após o ponto de cruzamento tem chance de ser censurado. Como consequência, quando calculamos a estatística de Log-rank Ponderado, temos menos cancelamento de diferenças positivas e negativas entre as duas funções intensidade estimadas.

\footnotetext{
${ }^{6}$ Cramér-von Mises.
} 


\begin{tabular}{|c|c|c|c|c|c|c|c|c|c|c|c|}
\hline \multirow{3}{*}{ Peso } & \multirow{3}{*}{ TA } & \multicolumn{7}{|c|}{ Resultados para cruzamento das funções de intensidade na ausência de censura } & \multirow{3}{*}{ TA } & \multirow{2}{*}{\multicolumn{2}{|c|}{ Estatísticas }} \\
\hline & & \multicolumn{2}{|c|}{ Estatísticas } & \multirow[t]{2}{*}{ Peso } & \multirow[t]{2}{*}{ TA } & \multicolumn{2}{|c|}{ Estatísticas } & \multirow[t]{2}{*}{ Peso } & & & \\
\hline & & LRP & CVM & & & LRP & $\mathbf{C V M}^{6}$ & & & LRP & CVM \\
\hline \multirow[t]{11}{*}{ Log-Rank } & 10 & 0,352 & 0,499 & \multirow[t]{11}{*}{ Gehan } & 10 & 0,463 & 0,649 & Fleming & 10 & 0,392 & 0,565 \\
\hline & 15 & 0,343 & 0,610 & & 15 & 0,556 & 0,797 & $\mathbf{e}$ & 15 & 0,445 & 0,704 \\
\hline & 20 & 0,367 & 0,716 & & 20 & 0,632 & 0,889 & Harrington & 20 & 0,539 & 0,822 \\
\hline & 30 & 0,399 & 0,860 & & 30 & 0,773 & 0,971 & $\gamma=0,5 \mathbf{e} \rho=2$ & 30 & 0,670 & 0,933 \\
\hline & 40 & 0,453 & 0,942 & & 40 & 0,861 & 0,994 & & 40 & 0,778 & 0,98 \\
\hline & 45 & 0,473 & 0,961 & & 45 & 0,895 & 0,997 & & 45 & 0,819 & 0,988 \\
\hline & 50 & 0,486 & 0,979 & & 50 & 0,923 & 0,999 & & 50 & 0,849 & 0,993 \\
\hline & 60 & 0,535 & 0,992 & & 60 & 0,958 & 1 & & 60 & 0,898 & 0,998 \\
\hline & 80 & 0,621 & 0,999 & & 80 & 0,988 & 1 & & 80 & 0,961 & 1 \\
\hline & 100 & 0,691 & 1 & & 100 & 0,996 & 1 & & 100 & 0,986 & 1 \\
\hline & 150 & 0,815 & 1 & & 150 & 1 & 1 & & 150 & 0,999 & 1 \\
\hline \multirow{11}{*}{$\begin{array}{c}\text { Tarone } \\
\text { e } \\
\text { Ware } \\
\gamma=0,5\end{array}$} & 10 & 0,088 & 0,193 & \multirow{11}{*}{$\begin{array}{l}\text { Peto } \\
\text { e } \\
\text { Peto }\end{array}$} & 10 & 0,372 & 0,558 & Peto & 10 & 0,244 & 0,366 \\
\hline & 15 & 0,089 & 0,212 & & 15 & 0,441 & 0,710 & e & 15 & 0,442 & 0,708 \\
\hline & 20 & 0,097 & 0,250 & & 20 & 0,511 & 0,821 & Peto & 20 & 0,513 & 0,823 \\
\hline & 30 & 0,114 & 0,334 & & 30 & 0,644 & 0,939 & Modificado & 30 & 0,648 & 0,939 \\
\hline & 40 & 0,130 & 0,425 & & 40 & 0,752 & 0,983 & & 40 & 0,757 & 0,982 \\
\hline & 45 & 0,147 & 0,481 & & 45 & 0,797 & 0,991 & & 45 & 0,794 & 0,990 \\
\hline & 50 & 0,155 & 0,523 & & 50 & 0,837 & 0,996 & & 50 & 0,832 & 0,995 \\
\hline & 60 & 0,181 & 0,62 & & 60 & 0,888 & 0,999 & & 60 & 0,893 & 0,999 \\
\hline & 80 & 0,224 & 0,81 & & 80 & 0,953 & 1 & & 80 & 0,956 & 1 \\
\hline & 100 & 0,277 & 0,92 & & 100 & 0,981 & 1 & & 100 & 0,982 & 1 \\
\hline & 150 & 0,373 & 1 & & 150 & 0,999 & 1 & & 150 & 0,999 & 1 \\
\hline
\end{tabular}

Tabela 4.24: Tabela da função poder para caso discreto $\left(W_{1} \sim \operatorname{Poi}(\lambda=1)\right.$ e $\left.W_{2} \sim \operatorname{Geo}(p=0,3)\right)$ sem censura.
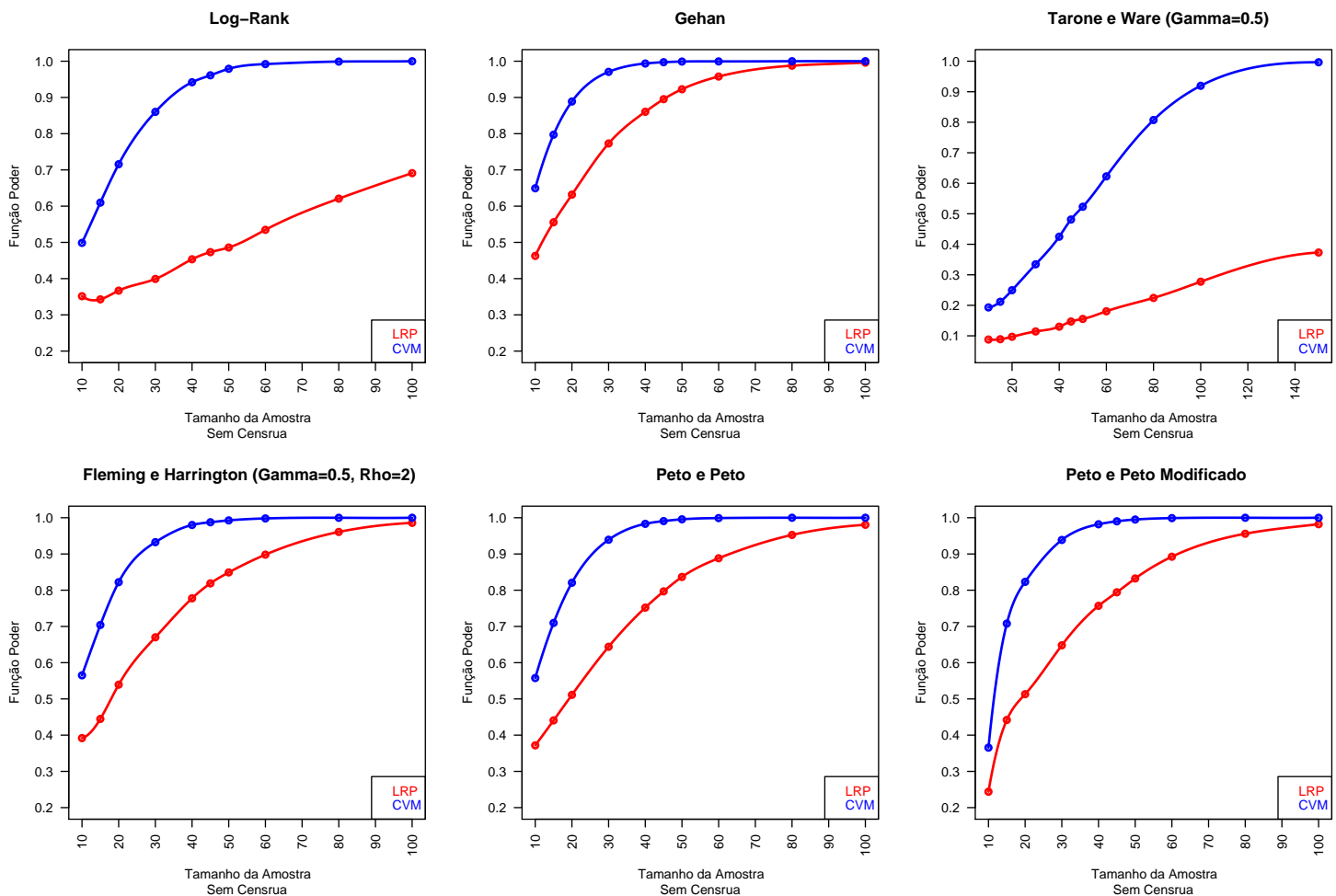

Figura 4.26: Gráfico do função poder para caso discreto $\left(W_{1} \sim \operatorname{Poi}(\lambda=1)\right.$ e $\left.W_{2} \sim \operatorname{Geo}(p=0,3)\right)$ sem censura. 


\begin{tabular}{|c|c|c|c|c|c|c|c|c|c|c|c|c|c|}
\hline \multirow{3}{*}{ Peso } & \multirow{3}{*}{ TA } & \multicolumn{11}{|c|}{ Resultados para cruzamento das funções de intensidade na presença de censura } & \multirow{3}{*}{$\%$ Cens 2} \\
\hline & & \multicolumn{2}{|c|}{ Estatísticas } & \multirow[t]{2}{*}{ Peso } & \multirow[t]{2}{*}{ TA } & \multicolumn{2}{|c|}{ Estatísticas } & \multirow[t]{2}{*}{ Peso } & \multirow[t]{2}{*}{ TA } & \multicolumn{2}{|c|}{ Estatísticas } & \multirow[t]{2}{*}{$\%$ Cens1 } & \\
\hline & & LRP & CVM & & & LRP & CVM & & & LRP & CVM & & \\
\hline \multirow{11}{*}{ Log-Rank } & 10 & 0,085 & 0,094 & Gehan & 10 & 0,099 & 0,118 & Fleming & 10 & 0,085 & 0,102 & & \\
\hline & 15 & 0,602 & 0,667 & & 15 & 0,685 & 0,807 & e & 15 & 0,602 & 0,719 & & \\
\hline & 20 & 0,707 & 0,762 & & 20 & 0,792 & 0,889 & Harrington & 20 & 0,707 & 0,815 & & \\
\hline & 30 & 0,863 & 0,902 & & 30 & 0,915 & 0,970 & $\gamma=0,5 \mathbf{e} \rho=2$ & 30 & 0,863 & 0,936 & & \\
\hline & 40 & 0,939 & 0,964 & & 40 & 0,968 & 0,993 & & 40 & 0,939 & 0,98 & 26,66 & 42,12 \\
\hline & 45 & 0,961 & 0,979 & & 45 & 0,983 & 0,997 & & 45 & 0,961 & 0,990 & & \\
\hline & 50 & 0,975 & 0,986 & & 50 & 0,989 & 0,999 & & 50 & 0,975 & 0,994 & & \\
\hline & 60 & 0,992 & 0,997 & & 60 & 0,997 & 1 & & 60 & 0,992 & 0,999 & & \\
\hline & 80 & 0,998 & 1,000 & & 80 & 1 & 1 & & 80 & 0,998 & 1 & & \\
\hline & 100 & 1 & 1 & & 100 & 1 & 1 & & 100 & 1 & 1 & & \\
\hline & 150 & 1 & 1 & & 150 & 1 & 1 & & 150 & 1 & 1 & & \\
\hline Tarone & 10 & 0,145 & 0,191 & Peto & 10 & 0,085 & 0,103 & Peto & 10 & 0,085 & 0,103 & & \\
\hline e & 15 & 0,157 & 0,226 & e & 15 & 0,602 & 0,723 & $\mathbf{e}$ & 15 & 0,602 & 0,723 & & \\
\hline Ware & 20 & 0,190 & 0,272 & Peto & 20 & 0,707 & 0,822 & Peto & 20 & 0,707 & 0,823 & & \\
\hline \multirow[t]{8}{*}{$\gamma=0,5$} & 30 & 0,246 & 0,362 & & 30 & 0,863 & 0,941 & Modificado & 30 & 0,863 & 0,941 & 26,66 & 42,12 \\
\hline & 40 & 0,302 & 0,457 & & 40 & 0,939 & 0,982 & & 40 & 0,939 & 0,982 & & \\
\hline & 45 & 0,329 & 0,498 & & 45 & 0,961 & 0,991 & & 45 & 0,961 & 0,991 & & \\
\hline & 50 & 0,352 & 0,548 & & 50 & 0,975 & 0,996 & & 50 & 0,975 & 0,996 & & \\
\hline & 60 & 0,403 & 0,646 & & 60 & 0,992 & 0,999 & & 60 & 0,992 & 0,999 & & \\
\hline & 80 & 0,512 & 0,806 & & 80 & 0,998 & 1 & & 80 & 0,998 & 1 & & \\
\hline & 100 & 0,600 & 0,906 & & 100 & 1 & 1 & & 100 & 1 & 1 & & \\
\hline & 150 & 0,773 & 0,992 & & 150 & 1 & 1 & & 150 & 1 & 1 & & \\
\hline
\end{tabular}

Tabela 4.25: Tabela da função poder para caso discreto $\left(W_{1} \sim \operatorname{Poi}(\lambda=1)\right.$ e $\left.W_{2} \sim \operatorname{Geo}(p=0.3)\right)$ com censura 2 à direita.
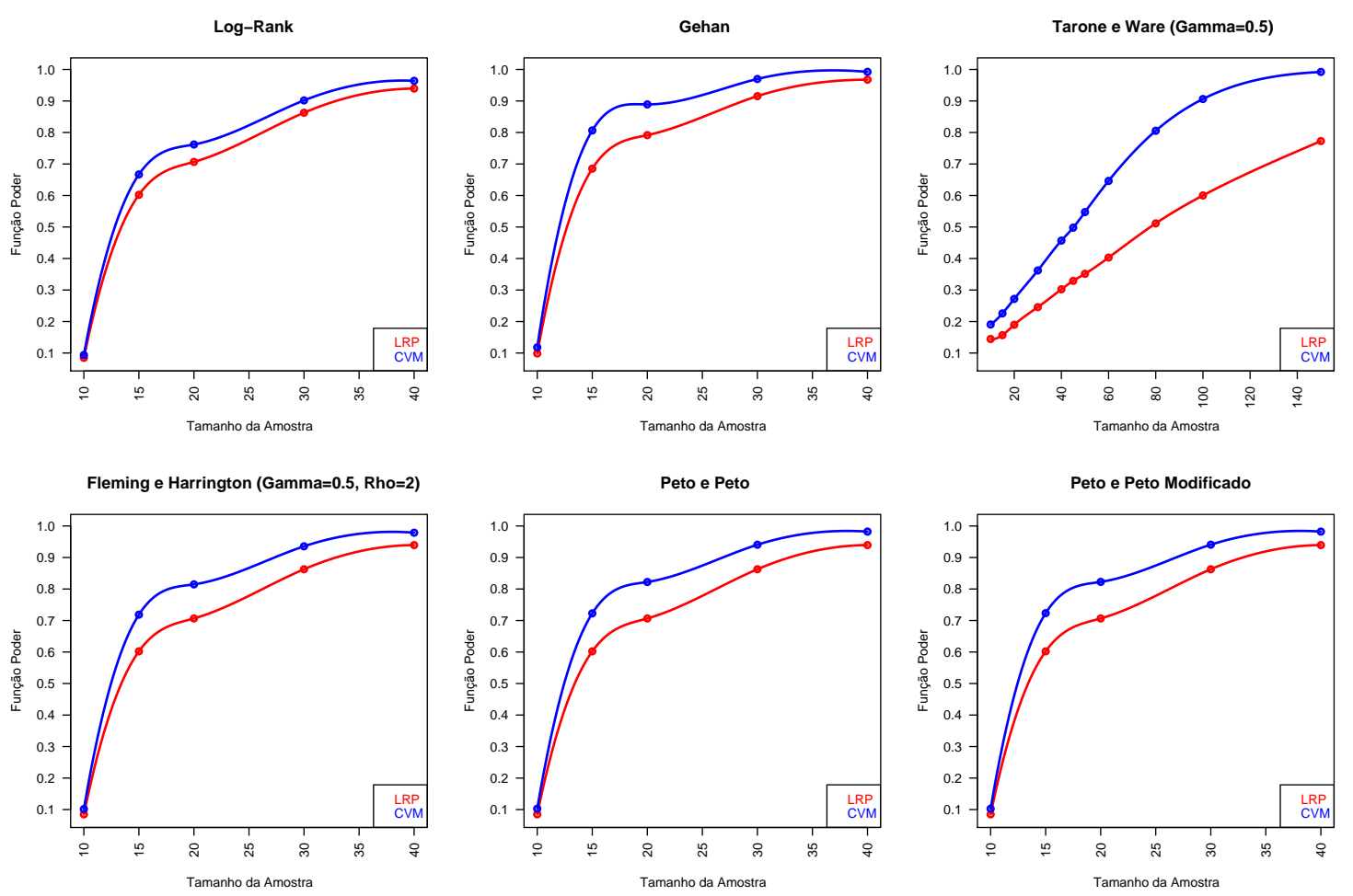

Figura 4.27: Gráfico do função poder para caso discreto $\left(W_{1} \sim \operatorname{Poi}(\lambda=1)\right.$ e $\left.W_{2} \sim \operatorname{Geo}(p=0.3)\right)$ com censura 2 à direita.

\subsection{Cenário geral para dados discretos}

O intuito desta seção é mostrar um cenário geral das estatísticas estudas neste trabalho. Para isto, fixamos a função de intensidade do grupo de controle em $h^{n_{1}}(\ell)=0,15 \forall \ell \in\{1,2, \ldots, 10\}$, e a função 
de intensidade para o grupo de tratamento é $h^{n_{2}}(\ell)=\beta_{0}+\beta_{1} \ell, \quad \ell \in\{1,2, \ldots, 10\}$ sendo $\beta_{0}$ e $\beta_{1}$ dois parâmetros que são usados para tratar casos importantes na avaliação de situações que acontecem com as funções de intensidade. Neste experimento, como temos apenas dez categorias, vamos adicionar o teste Log-rank Modificado na comparação com os testes de Log-rank Ponderado, Cramér-von Mises e o de Renyi. Assim, dividimos o experimento em quatro casos:

\section{Taxas de Falha}

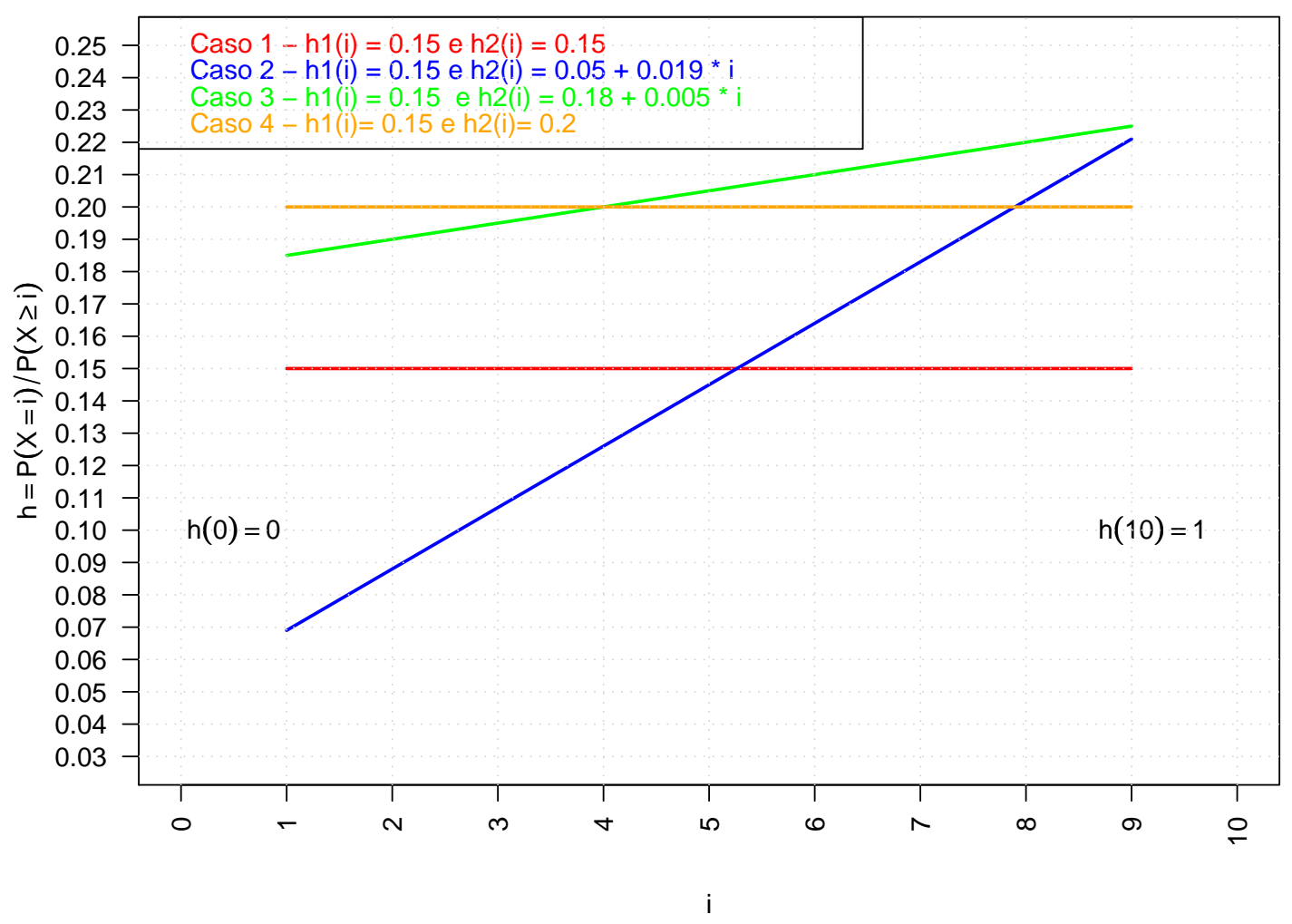

Figura 4.28: Gráfico do cenário geral para dados discretos.

(1) Para $\beta_{0}=0,15$ e $\beta_{1}=0$, obtemos,

$$
h^{n_{2}}(\ell)=0,15, \quad \forall \ell \in\{1,2, \ldots, 10\}
$$

(2) Para $\beta_{0}=0,05$ e $\beta_{1}=0,019$, obtemos,

$$
h^{n_{2}}(\ell)=0,05+0,019 \ell, \quad \ell \in\{1,2, \ldots, 10\}
$$

(3) Para $\beta_{0}=0,18$ e $\beta_{1}=0,005$, obtemos,

$$
h^{n_{2}}(\ell)=0,18+0,005 \ell, \quad \ell \in\{1,2, \ldots, 10\}
$$


(4) Para $\beta_{0}=0,2, \beta_{1}=0$, obtemos,

$$
h^{n_{2}}(\ell)=0,2, \quad \forall \ell \in\{1,2, \ldots, 10\}
$$

Na situação de cruzamento das funções de intensidade, ou seja, no caso (2), temos que o cruzamento acontece em $\ell=5$. A seguir apresentamos o algoritmo.

1. Fixamos um nível de significância $\alpha=0,05$;

2. Definimos 2 populações com distribuição Multinomial:

- $X^{(1)}, \ldots, X^{(J)} \sim M\left(T A, p_{1}, p_{2}, \ldots, p_{10}\right)$;

- Para o caso com censura, temos $C^{(1)}, \ldots, C^{(J)} \sim U[1,10]$ truncado;

- A função de intensidade para a população de controle $h^{n_{1}}(\ell)=0,15 \forall \ell \in\{1,2, \ldots, 10\}$ e a função de intensidade é $h^{n_{2}}(\ell)=\beta_{0}+\beta_{1} \ell, \quad \ell \in\{1,2, \ldots, 10\}$ sendo $\beta_{0}$ e $\beta_{1}$ dois parâmetros escolhidos em um dos casos (1 a 4);

- À partir das funções de intensidade, calculamos as probabilidade $p_{1}, \ldots, p_{9}$ e $p_{10}$ usando a equação (2.5), ou seja, $p_{i}=P[W=i]=h(i) \prod_{\ell=1}^{i-1}[1-h(\ell)]$ dado que a hipótese nula é falsa (verdadeira no caso 1);

- Escolhemos uma das funções de ponderação descrita na tabela (3.1);

3. Realizamos o teste de hipótese usando valor crítico ao nível de significância $\alpha$;

4. Para estimarmos a função poder tomamos

$$
y_{m}= \begin{cases}1, & \text { se } H_{0} \text { foi rejeitada ao nível de significância } \alpha \\ 0, & \text { caso contrário }\end{cases}
$$

Obs: Verificamos se cometeu o erro do tipo I no caso 1.

5. Repetimos as etapas 2 a $4 m=10000$ vezes;

6. Estimamos o poder do teste na seguinte forma:

$$
\text { Poder }=\frac{1}{m} \sum_{k=1}^{m} y_{k}
$$




\begin{tabular}{|c|c|c|c|c|c|c|c|c|c|c|c|c|c|c|c|}
\hline \multicolumn{16}{|c|}{ Caso 1 sem censura } \\
\hline TA & Peso & LRP & CVM & MLR & Renyi & Peso & LRP & CVM & MLR & Renyi & Peso & LRP & CVM & MLR & Renyi \\
\hline 50 & Log-Rank & 0,0521 & 0,0573 & 0,0433 & 0,0351 & Tarone & 0,0555 & 0,0558 & 0,0433 & 0,0365 & Peto & 0,0546 & 0,0563 & 0,0433 & 0,0388 \\
\hline 75 & & 0,0494 & 0,0548 & 0,0522 & 0,0356 & e & 0,0548 & 0,0566 & 0,0522 & 0,0379 & e & 0,0521 & 0,056 & 0,0522 & 0,0362 \\
\hline 100 & & 0,049 & 0,0488 & 0,0498 & 0,0337 & Ware & 0,051 & 0,0476 & 0,0498 & 0,0328 & Peto & 0,0473 & 0,0514 & 0,0498 & 0,0337 \\
\hline 125 & & 0,0533 & 0,0519 & 0,0521 & 0,037 & $\gamma=0,5$ & 0,0485 & 0,048 & 0,0521 & 0,032 & & 0,0515 & 0,049 & 0,0521 & 0,035 \\
\hline 200 & & 0,0521 & 0,0502 & 0,0499 & 0,0302 & & 0,0502 & 0,0495 & 0,0499 & 0,0354 & & 0,0511 & 0,0512 & 0,0499 & 0,039 \\
\hline 50 & Gehan & 0,0557 & 0,0552 & 0,0433 & 0,0381 & Fleming & 0,0544 & 0,0566 & 0,0433 & 0,0376 & Peto & 0,0547 & 0,0563 & 0,0433 & 0,0363 \\
\hline 75 & & 0,0523 & 0,0566 & 0,0522 & 0,0375 & e & 0,0515 & 0,0546 & 0,0522 & 0,0331 & e & 0,0523 & 0,0558 & 0,0522 & 0,0337 \\
\hline 100 & & 0,049 & 0,0514 & 0,0498 & 0,035 & Harrington & 0,0488 & 0,0514 & 0,0498 & 0,0329 & Peto & 0,0471 & 0,0514 & 0,0498 & 0,0349 \\
\hline 125 & & 0,0516 & 0,0488 & 0,0521 & 0,0345 & $\gamma=0,5 \mathbf{e} \rho=2$ & 0,0519 & 0,049 & 0,0521 & 0,036 & Modificado & 0,0516 & 0,049 & 0,0521 & 0,0344 \\
\hline 200 & & 0,051 & 0,0519 & 0,0499 & 0,0354 & & 0,0512 & 0,0501 & 0,0499 & 0,0354 & & 0,051 & 0,0512 & 0,0499 & 0,0354 \\
\hline
\end{tabular}

Tabela 4.26: Tratamento versus Controle para o caso 1 sem censura.
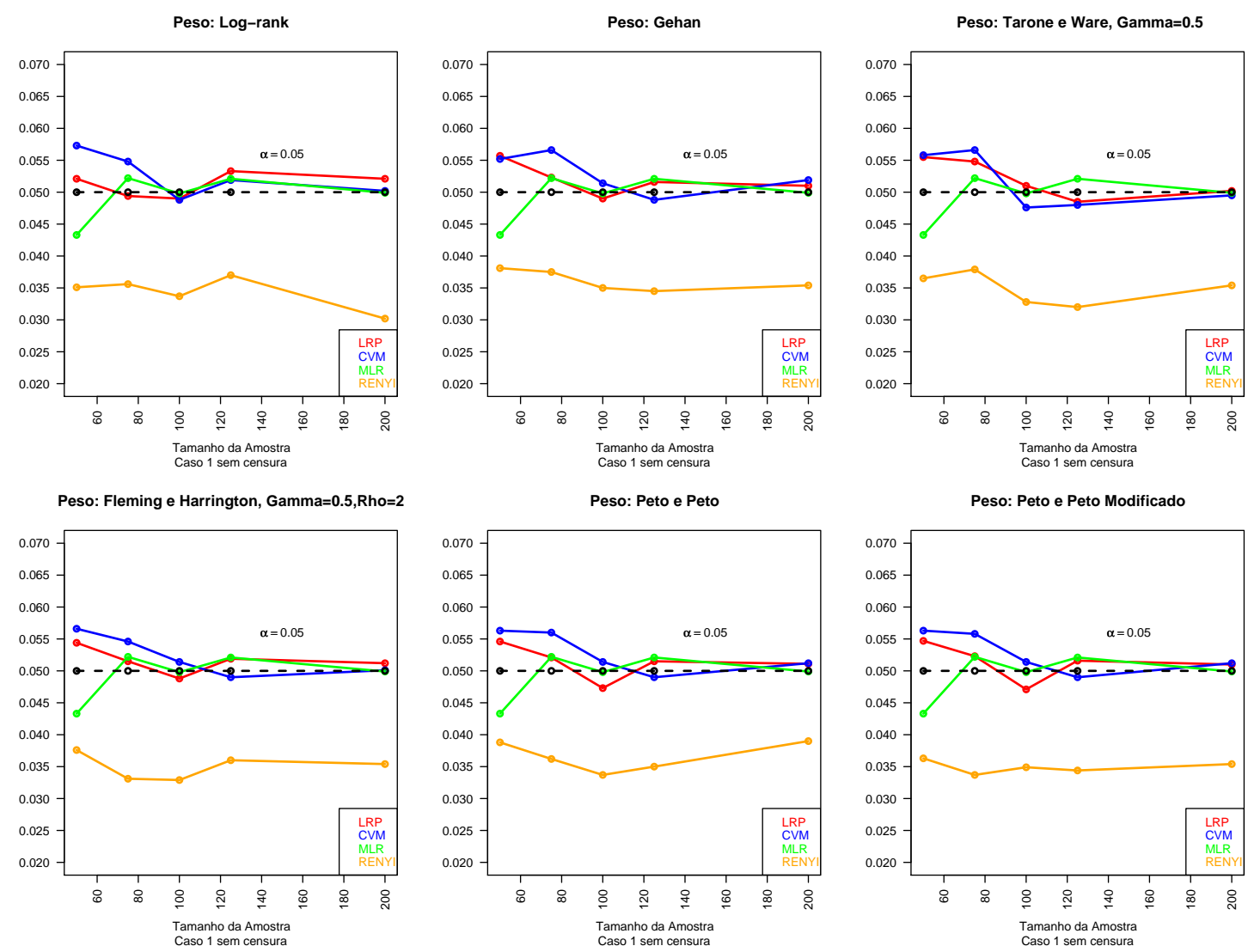

Figura 4.29: Tratamento versus Controle para o caso 1 sem censura.

Os resultados obtidos na tabela (4.26) são referentes ao caso 1 em que estamos sob a hipótese nula, no qual $W^{n_{1}}$ e $W^{n_{2}}$ tem funções de intensidade dadas por $h^{n_{1}}(\ell)=h^{n_{2}}(\ell)=0,15, \quad \forall \ell=1, \cdots, 10$ na ausência de censura. Um ponto importante dos resultados obtidos foi que a estatística de Renyi não se aproxima do nível de significância nominal de 0,05 . 


\begin{tabular}{|c|c|c|c|c|c|c|c|c|c|c|c|c|c|c|c|c|c|}
\hline \multicolumn{18}{|c|}{ Caso 1 com censura } \\
\hline TA & Peso & LRP & CVM & MLR & Renyi & Peso & LRP & CVM & MLR & Renyi & Peso & LRP & CVM & MLR & Renyi & \%Cens1 & $\%$ Cens \\
\hline 50 & Log-Rank & 0,0497 & 0,0538 & 0,0534 & 0,0312 & Tarone & 0,0508 & 0,0538 & 0,0534 & 0,0315 & Peto & 0,0507 & 0,054 & 0,0534 & 0,0323 & & \\
\hline 75 & & 0,049 & 0,0532 & 0,0522 & 0,0299 & e & 0,0498 & 0,052 & 0,0522 & 0,0301 & e & 0,0492 & 0,0519 & 0,0522 & 0,0287 & & \\
\hline 100 & & 0,0548 & 0,0564 & 0,0489 & 0,0351 & Ware & 0,0555 & 0,0568 & 0,0489 & 0,0349 & Peto & 0,0556 & 0,0556 & 0,0489 & 0,0343 & 43,92 & 43,8 \\
\hline 125 & & 0,0511 & 0,0513 & 0,0512 & 0,0304 & $\gamma=0,5$ & 0,0499 & 0,0505 & 0,0512 & 0,0299 & & 0,0501 & 0,0502 & 0,0512 & 0,0304 & & \\
\hline 200 & & 0,0526 & 0,0498 & 0,0489 & 0,0315 & & 0,0524 & 0,0516 & 0,0489 & 0,0319 & & 0,0522 & 0,0509 & 0,0489 & 0,032 & & \\
\hline 50 & Gehan & 0,0514 & 0,0524 & 0,0534 & 0,0317 & Fleming & 0,0506 & 0,0548 & 0,0534 & 0,0311 & Peto & 0,0507 & 0,054 & 0,0534 & 0,0323 & & \\
\hline 75 & & 0,0497 & 0,0519 & 0,0522 & 0,0298 & & 0,0492 & 0,0519 & 0,0522 & 0,0291 & e & 0,0493 & 0,0517 & 0,0522 & 0,0286 & & \\
\hline 100 & & 0,0541 & 0,0566 & 0,0489 & 0,0336 & Harrington & 0,0549 & 0,056 & 0,0489 & 0,035 & Peto & 0,0556 & 0,0556 & 0,0489 & 0,0343 & 43,92 & 43,8 \\
\hline 125 & & 0,0495 & 0,0504 & 0,0512 & 0,0301 & $\gamma=0,5 \mathbf{e} \rho=2$ & 0,0503 & 0,0504 & 0,0512 & 0,0303 & Modificado & 0,0501 & 0,0503 & 0,0512 & 0,0304 & & \\
\hline 200 & & 0,0518 & 0,0523 & 0,0489 & 0,0318 & & 0,0532 & 0,0508 & 0,0489 & 0,0322 & & 0,0522 & 0,0509 & 0,0489 & 0,032 & & \\
\hline
\end{tabular}

Tabela 4.27: Tratamento versus Controle para o caso 1 com censura.

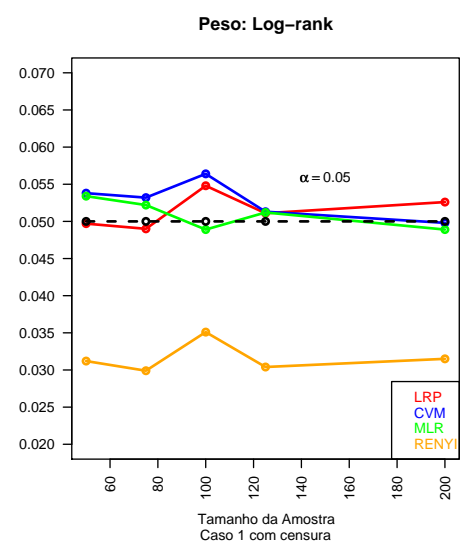

Peso: Fleming e Harrington, Gamma $=0.5$, Rho $=2$

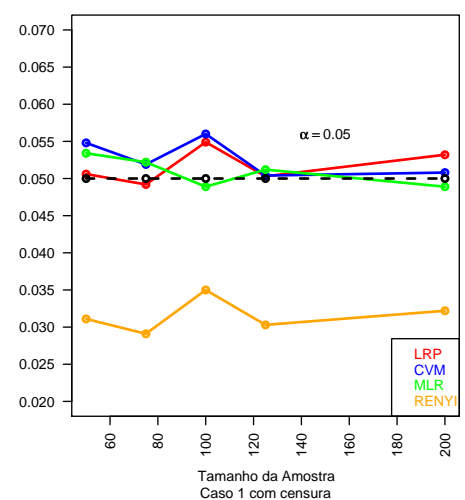

Peso: Gehan

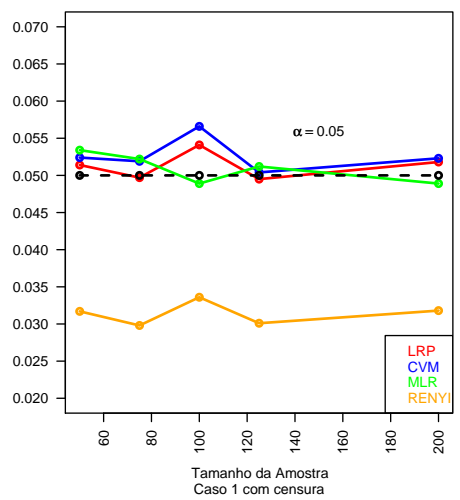

Peso: Peto e Peto

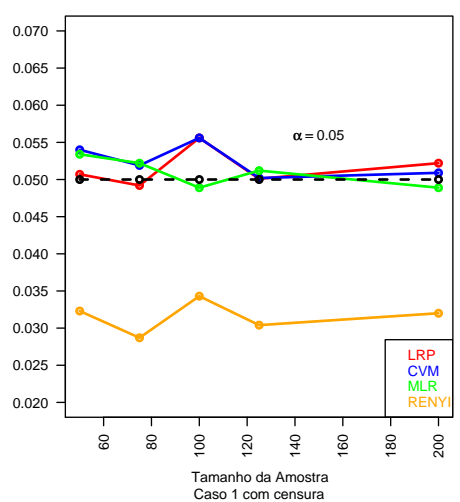

Peso: Tarone e Ware, Gamma=0.5

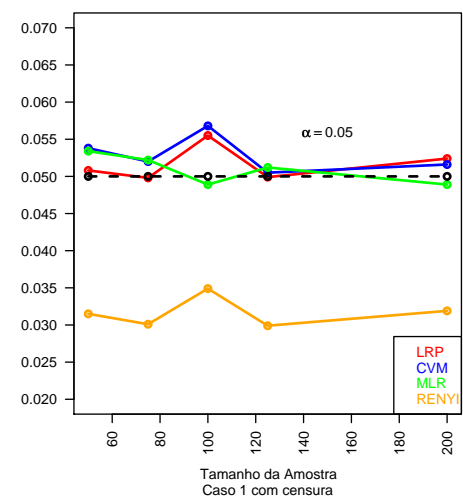

Peso: Peto e Peto Modificado

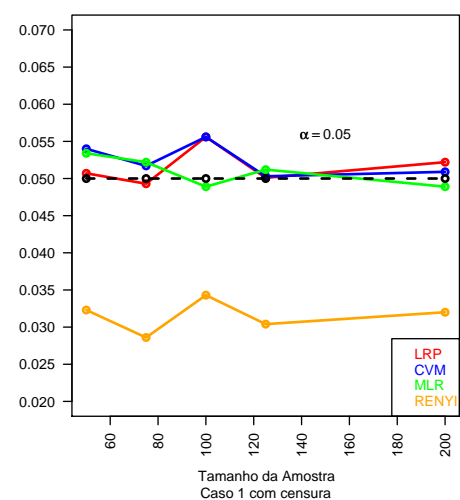

Figura 4.30: Tratamento versus Controle para o caso 1 com censura.

Os resultados obtidos na tabela (4.27) são referentes ao caso 1 e $W^{n_{1}}$ e $W^{n_{2}}$ que agora estão na presença de censura. Aqui, utilizamos censura aleatória com distribuição uniforme, no qual são censurados cerca de $44 \%$ dos dados para cada população. Assim, como nos resultados obtidos na ausência de censura, a estatística de Renyi também não se aproxima do nível de significância nominal de 0,05 quando estamos na presença de censura. Com os resultados obtidos nas duas situações, excluímos o teste de Renyi da nossa comparação em relação aos casos 2 a 4. 


\begin{tabular}{|c|c|c|c|c|c|c|c|c|c|c|c|c|}
\hline \multicolumn{13}{|c|}{ Caso 2 sem censura } \\
\hline TA & Peso & LRP & CVM & MLR & Peso & LRP & CVM & MLR & Peso & LRP & CVM & MLR \\
\hline 50 & Log-Rank & 0,1366 & 0,3066 & 0,2408 & Tarone & 0,1998 & 0,3682 & 0,2408 & Peto & 0,2401 & 0,3889 & 0,2408 \\
\hline 75 & & 0,1758 & 0,4281 & 0,3227 & e & 0,2721 & 0,5133 & 0,3227 & e & 0,3417 & 0,54 & 0,3227 \\
\hline 100 & & 0,2147 & 0,5377 & 0,4106 & Ware & 0,3347 & 0,6291 & 0,4106 & Peto & 0,421 & 0,6592 & 0,4106 \\
\hline 125 & & 0,2517 & 0,6363 & 0,5157 & $\gamma=0,5$ & 0,412 & 0,7315 & 0,5157 & & 0,5154 & 0,7602 & 0,5157 \\
\hline 200 & & 0,3718 & 0,8396 & 0,7591 & & 0,5884 & 0,9079 & 0,7591 & & 0,7103 & 0,9258 & 0,7591 \\
\hline 50 & Gehan & 0,2532 & 0,3945 & 0,2408 & Fleming & 0,2274 & 0,37 & 0,2408 & Peto & 0,2416 & 0,3896 & 0,2408 \\
\hline 75 & & 0,3611 & 0,5448 & 0,3227 & e & 0,3182 & 0,5168 & 0,3227 & e & 0,3431 & 0,5401 & 0,3227 \\
\hline 100 & & 0,4437 & 0,6696 & 0,4106 & Harrington & 0,3911 & 0,6326 & 0,4106 & Peto & 0,4218 & 0,6601 & 0,4106 \\
\hline 125 & & 0,54 & 0,7659 & 0,5157 & $\gamma=0,5 \mathbf{e} \rho=2$ & 0,4768 & 0,7344 & 0,5157 & Modificado & 0,5158 & 0,7607 & 0,5157 \\
\hline 200 & & 0,7365 & 0,9299 & 0,7591 & & 0,6679 & 0,9097 & 0,7591 & & 0,7109 & 0,9258 & 0,7591 \\
\hline
\end{tabular}

Tabela 4.28: Tratamento versus Controle para o caso 2 sem censura.

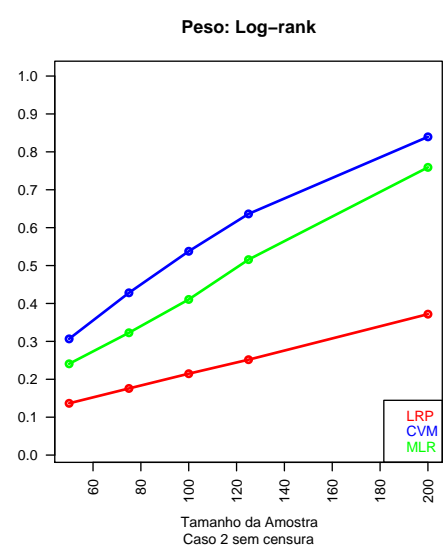

Peso: Fleming e Harrington, Gamma=0.5,Rho=2

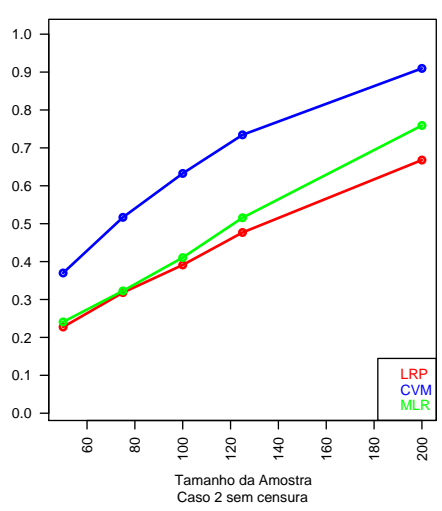

Peso: Gehan

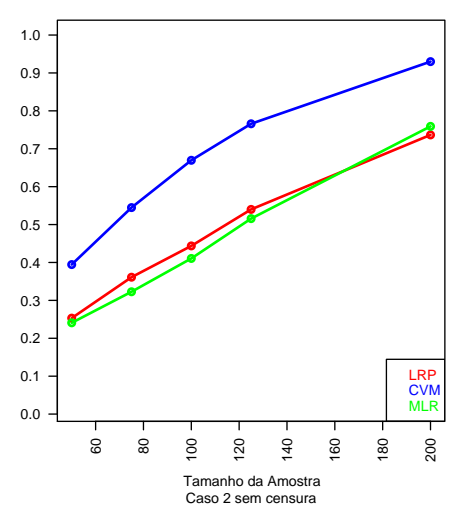

Peso: Peto e Peto

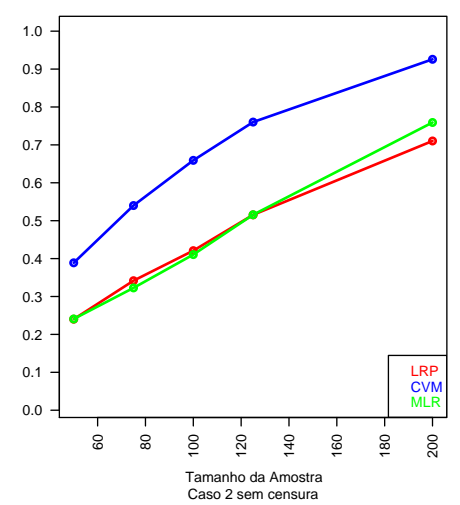

Peso: Tarone e Ware, Gamma $=0.5$

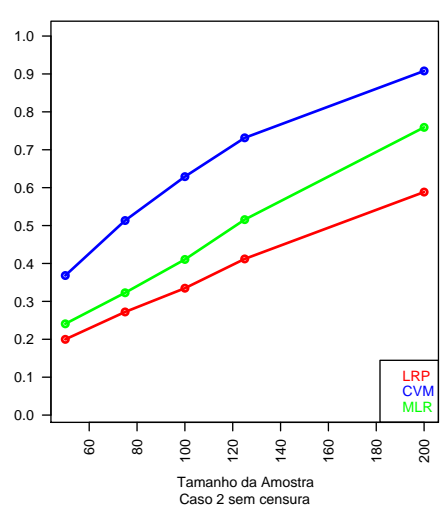

Peso: Peto e Peto Modificado

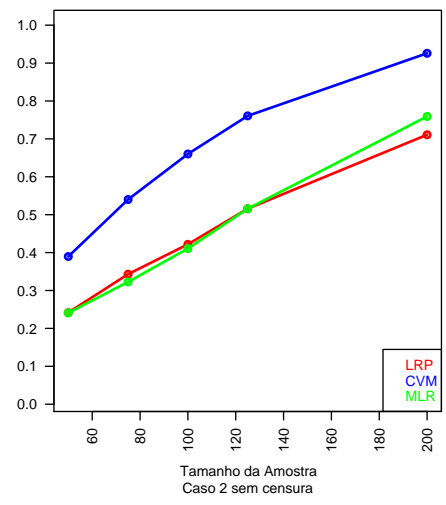

Figura 4.31: Tratamento versus Controle para o caso 2 sem censura.

Na tabela (4.28) temos resultados do caso 2 em que $W^{n_{1}}$ tem taxa constante $h^{n_{1}}(\ell)=0,15$ e $W^{n_{2}}$ tem taxa linear $h^{n_{2}}(\ell)=0,05+0,019 \ell$, para as duas taxas temos $\ell=1, \cdots, 10$ na ausência de censura. Nos resultados obtidos, notamos que em todos os processos de ponderação utilizados, CVM é mais poderosa que LRP e MLR, exceto para o peso $\log$-rank $\left(u\left(n^{\star}, \ell\right)=1\right)$ em que MLR têm comportamento similar ao CVM. Apesar de MLR ter um bom desempenho quando cruzamos as funções de intensidade, o fato de aumentarmos o número de categorias (10 categorias) em relação ao experimento dos casos finitos, pode ser a causa na diminuição do poder em relação ao CVM. 


\begin{tabular}{|c|c|c|c|c|c|c|c|c|c|c|c|c|c|c|}
\hline \multirow[b]{2}{*}{ TA } & \multirow[b]{2}{*}{ Peso } & \multirow[b]{2}{*}{ LRP } & \multirow[b]{2}{*}{ CVM } & \multirow[b]{2}{*}{ MLR } & \multirow[b]{2}{*}{ Peso } & \multicolumn{3}{|c|}{ Caso 2 com censura } & \multirow[b]{2}{*}{ Peso } & \multirow[b]{2}{*}{ LRP } & \multirow[b]{2}{*}{ CVM } & \multirow[b]{2}{*}{ MLR } & \multirow[b]{2}{*}{$\%$ Cens1 } & \multirow[b]{2}{*}{$\%$ Cens 2} \\
\hline & & & & & & LRP & CVM & MLR & & & & & & \\
\hline 50 & Log-Rank & 0,31 & 0,3733 & 0,2527 & Tarone & 0,3242 & 0,3826 & 0,2527 & Peto & 0,327 & 0,387 & 0,2527 & & \\
\hline 75 & & 0,4324 & 0,5267 & 0,3614 & e & 0,4516 & 0,5422 & 0,3614 & e & 0,4596 & 0,5445 & 0,3614 & & \\
\hline 100 & & 0,5442 & 0,6416 & 0,4674 & Ware & 0,5651 & 0,6546 & 0,4674 & Peto & 0,5742 & 0,6583 & 0,4674 & 43,2 & 53,3 \\
\hline 125 & & 0,639 & 0,7464 & 0,5711 & $\gamma=0,5$ & 0,6649 & 0,7604 & 0,5711 & & 0,6733 & 0,7647 & 0,5711 & & \\
\hline 200 & & 0,8256 & 0,9109 & 0,7892 & & 0,8458 & 0,9199 & 0,7892 & & 0,854 & 0,9221 & 0,7892 & & \\
\hline 50 & Gehan & 0,3337 & 0,3863 & 0,2527 & Fleming & 0,3128 & 0,3757 & 0,2527 & Peto & 0,327 & 0,3874 & 0,2527 & & \\
\hline 75 & & 0,4697 & 0,542 & 0,3614 & e & 0,4397 & 0,533 & 0,3614 & e & 0,4596 & 0,5444 & 0,3614 & & \\
\hline 100 & & 0,5833 & 0,656 & 0,4674 & Harrington & 0,5529 & 0,6483 & 0,4674 & Peto & 0,5742 & 0,6584 & 0,4674 & 43,2 & 53,3 \\
\hline 125 & & 0,6843 & 0,7629 & 0,5711 & $\gamma=0,5 \mathbf{e} \rho=2$ & 0,6492 & 0,7548 & 0,5711 & Modificado & 0,6736 & 0,7649 & 0,5711 & & \\
\hline 200 & & 0,8611 & 0,9211 & 0,7892 & & 0,8372 & 0,9155 & 0,7892 & & 0,8541 & 0,9221 & 0,7892 & & \\
\hline
\end{tabular}

Tabela 4.29: Tratamento versus Controle para o caso 2 com censura.

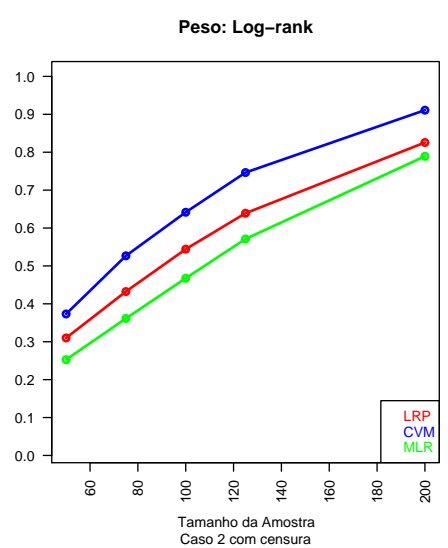

Peso: Fleming e Harrington, Gamma $=0.5, \mathrm{Rho}=2$

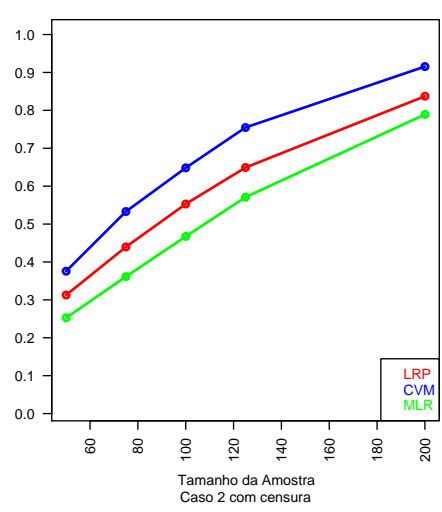

Peso: Gehan

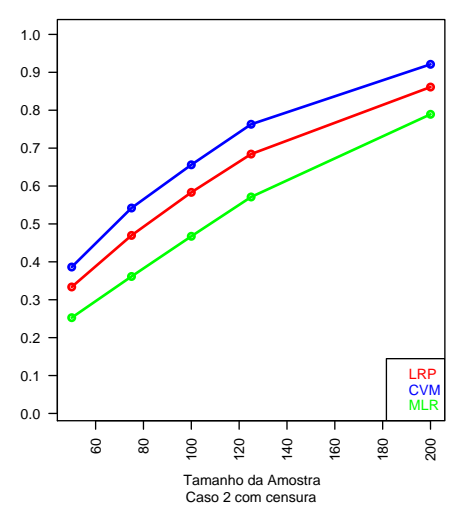

Peso: Peto e Peto

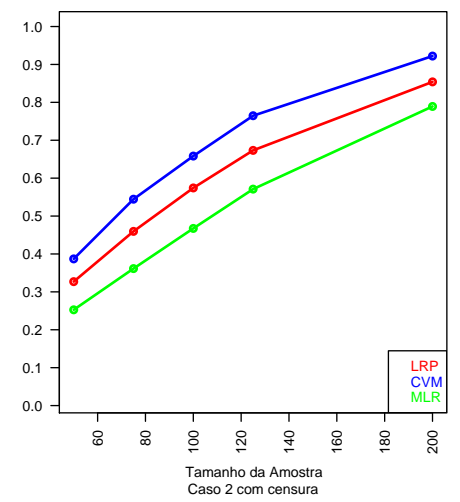

Peso: Tarone e Ware, Gamma=0.5

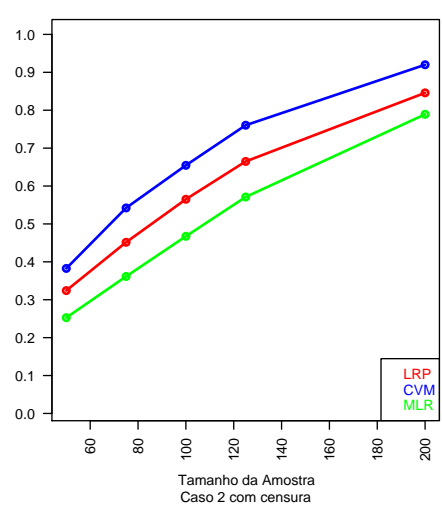

Peso: Peto e Peto Modificado

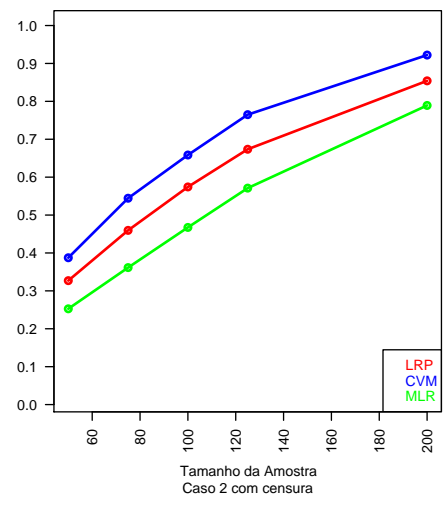

Figura 4.32: Tratamento versus Controle para o caso 2 com censura.

Na tabela (4.29) temos resumo dos resultados e neste caso, as duas funções de intensidade cruzam em $\ell=5$, em que as variáveis de interesse $W^{n_{1}}$ e $W^{n_{2}}$ tem $50 \%$ de chance de estar localizado antes do cruzamento e 50\% após. Em todos os processos de ponderação utilizados a estatística de CVM têm poder levemente maior que as demais. Neste experimento, estamos na presença de censura aleatória $C_{i} \sim$ $U[0,10]$ truncado, nesta situação temos que o ponto de cruzamento é antecipado para $\ell=4$ (devido à censura), diminuindo o cancelamento das diferenças positivas e negativas entre as duas funções intensidade estimadas. 


\begin{tabular}{|c|c|c|c|c|c|c|c|c|c|c|c|c|}
\hline \multicolumn{13}{|c|}{ Caso 3 sem censura } \\
\hline TA & Peso & LRP & CVM & MLR & Peso & LRP & CVM & MLR & Peso & LRP & CVM & MLR \\
\hline 50 & Log-Rank & 0,2134 & 0,2931 & 0,1732 & Tarone & 0,1623 & 0,2576 & 0,1732 & Peto & 0,1447 & 0,2346 & 0,1732 \\
\hline 75 & & 0,3012 & 0,4066 & 0,1935 & $\mathbf{e}$ & 0,2242 & 0,3592 & 0,1935 & e & 0,193 & 0,3254 & 0,1935 \\
\hline 100 & & 0,3822 & 0,5163 & 0,2359 & Ware & 0,2831 & 0,4622 & 0,2359 & Peto & 0,2413 & 0,4166 & 0,2359 \\
\hline 125 & & 0,4543 & 0,6098 & 0,2791 & $\gamma=0,5$ & 0,3298 & 0,5472 & 0,2791 & & 0,2836 & 0,4929 & 0,2791 \\
\hline 200 & & 0,6515 & 0,8044 & 0,4516 & & 0,4904 & 0,746 & 0,4516 & & 0,4165 & 0,6868 & 0,4516 \\
\hline 50 & Gehan & 0,1325 & 0,2253 & 0,1732 & Fleming & 0,1468 & 0,2239 & 0,1732 & Peto & 0,1442 & 0,234 & 0,1732 \\
\hline 75 & & 0,1773 & 0,3126 & 0,1935 & e & 0,1959 & 0,3133 & 0,1935 & e & 0,1925 & 0,3248 & 0,1935 \\
\hline 100 & & 0,2146 & 0,4003 & 0,2359 & Harrington & 0,2445 & 0,3971 & 0,2359 & Peto & 0,2408 & 0,416 & 0,2359 \\
\hline 125 & & 0,2502 & 0,4715 & 0,2791 & $\gamma=0,5 \mathbf{e} \rho=2$ & 0,2856 & 0,4708 & 0,2791 & Modificado & 0,2828 & 0,4922 & 0,2791 \\
\hline 200 & & 0,372 & 0,6627 & 0,4516 & & 0,4187 & 0,662 & 0,4516 & & 0,4159 & 0,6859 & 0,4516 \\
\hline
\end{tabular}

Tabela 4.30: Tratamento versus Controle para o caso 3 sem censura.

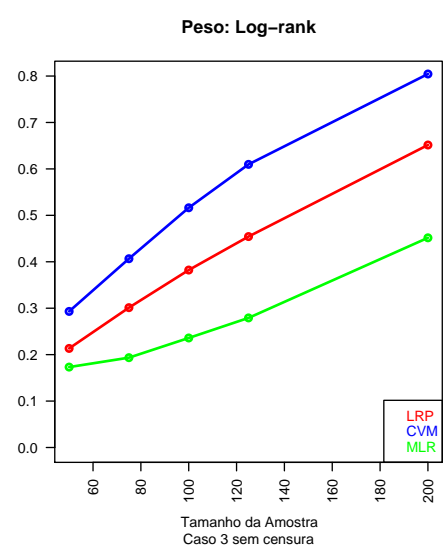

Peso: Fleming e Harrington, Gamma=0.5,Rho=2

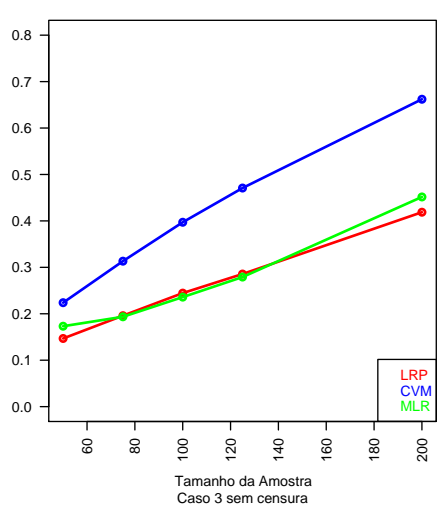

Peso: Gehan

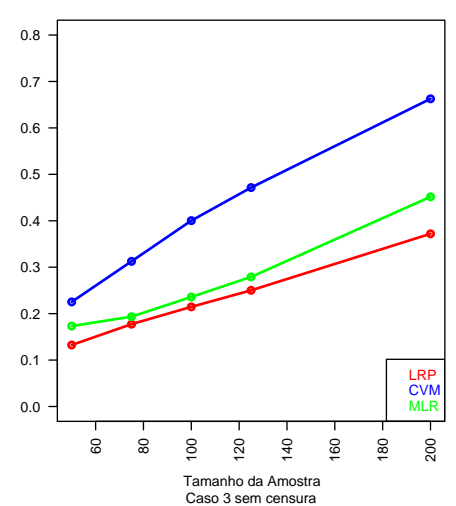

Peso: Peto e Peto

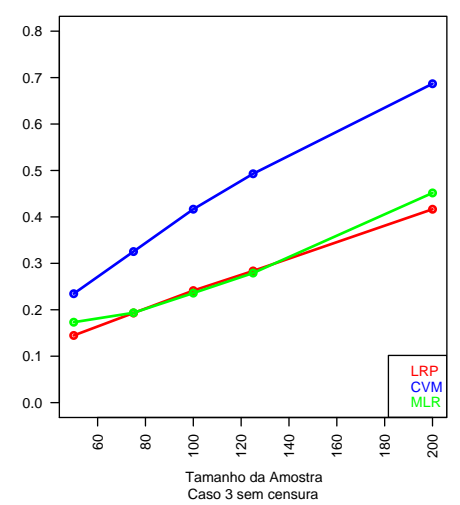

Peso: Tarone e Ware, Gamma $=0.5$

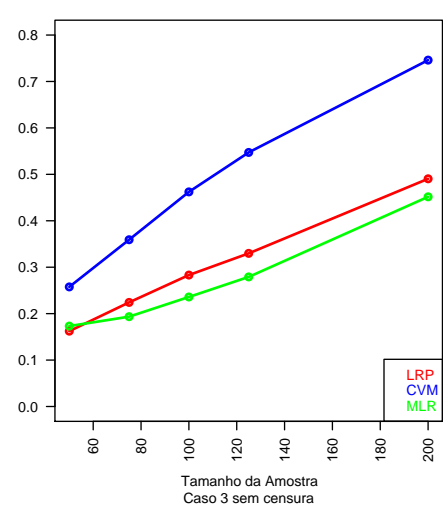

Peso: Peto e Peto Modificado

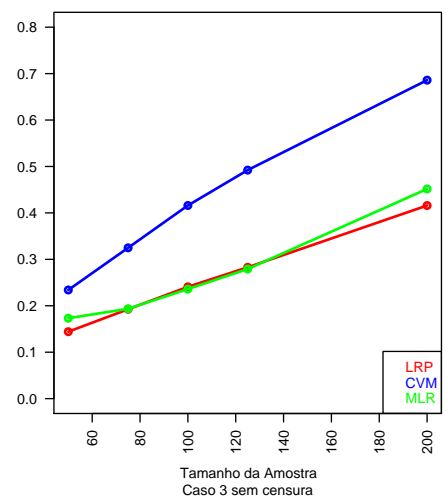

Figura 4.33: Tratamento versus Controle para o caso 3 sem censura.

Na tabela (4.30) temos resultados do caso 3 em que $W^{n_{1}}$ tem taxa constante $h^{n_{1}}(\ell)=0,15$ e $W^{n_{2}}$ tem taxa linear $h^{n_{2}}(\ell)=0,18+0,005 \ell$, para as duas taxas temos $\ell \in \mathcal{A}=\{1, \ldots, 10\} \subset \mathbb{N}$ na ausência de censura. Pelos resultados obtidos, notamos que, em todos os processos de ponderação utilizados a estatística CVM é mais poderosa que a de LRP e MLR, exceto no caso do peso log-rank $\left(u\left(n^{\star}, \ell\right)=1\right)$ em que LRP é melhor que MLR. 


\begin{tabular}{|c|c|c|c|c|c|c|c|c|c|c|c|c|c|c|}
\hline \multirow[b]{2}{*}{ TA } & \multirow[b]{2}{*}{ Peso } & \multirow[b]{2}{*}{ LRP } & \multirow[b]{2}{*}{ CVM } & \multirow[b]{2}{*}{ MLR } & \multirow[b]{2}{*}{ Peso } & \multicolumn{3}{|c|}{ Caso 3 com censura } & \multirow[b]{2}{*}{ Peso } & \multirow[b]{2}{*}{ LRP } & \multirow[b]{2}{*}{ CVM } & \multirow[b]{2}{*}{ MLR } & \multirow[b]{2}{*}{$\%$ Cens1 } & \multirow[b]{2}{*}{$\%$ Cens 2} \\
\hline & & & & & & LRP & CVM & MLR & & & & & & \\
\hline 50 & Log-Rank & 0,1426 & 0,1431 & 0,102 & Tarone & 0,1289 & 0,1389 & 0,102 & Peto & 0,1259 & 0,1362 & 0,102 & & \\
\hline 75 & & 0,1867 & 0,1825 & 0,12 & e & 0,1625 & 0,1771 & 0,12 & e & 0,1566 & 0,1722 & 0,12 & & \\
\hline 100 & & 0,2356 & 0,2283 & 0,1478 & Ware & 0,2077 & 0,2217 & 0,1478 & Peto & 0,1975 & 0,2174 & 0,1478 & 43,92 & 43,8 \\
\hline 125 & & 0,2833 & 0,2712 & 0,1759 & $\gamma=0,5$ & 0,2453 & 0,2639 & 0,1759 & & 0,2348 & 0,2557 & 0,1759 & & \\
\hline 200 & & 0,412 & 0,39 & 0,2628 & & 0,3555 & 0,3762 & 0,2628 & & 0,3385 & 0,3713 & 0,2628 & & \\
\hline 50 & Gehan & 0,1151 & 0,133 & 0,102 & Fleming & 0,1286 & 0,137 & 0,102 & Peto & 0,1256 & 0,1362 & 0,102 & & \\
\hline 75 & & 0,1437 & 0,168 & 0,12 & e & 0,1627 & 0,1735 & 0,12 & e & 0,1566 & 0,1722 & 0,12 & 43,92 & 43,8 \\
\hline 100 & & 0,1793 & 0,2121 & 0,1478 & Harrington & 0,2043 & 0,2179 & 0,1478 & Peto & 0,1973 & 0,2173 & 0,1478 & & \\
\hline 125 & & 0,2109 & 0,2531 & 0,1759 & $\gamma=0,5 \mathbf{e} \rho=2$ & 0,2427 & 0,2587 & 0,1759 & Modificado & 0,2347 & 0,2557 & 0,1759 & & \\
\hline 200 & & 0,3042 & 0,361 & 0,2628 & & 0,3522 & 0,3731 & 0,2628 & & 0,3383 & 0,3711 & 0,2628 & & \\
\hline
\end{tabular}

Tabela 4.31: Tratamento versus Controle para o caso 3 com censura.

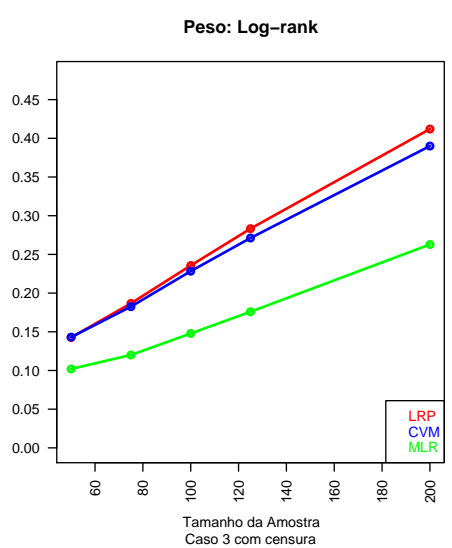

Peso: Fleming e Harrington, Gamma=0.5,Rho=2

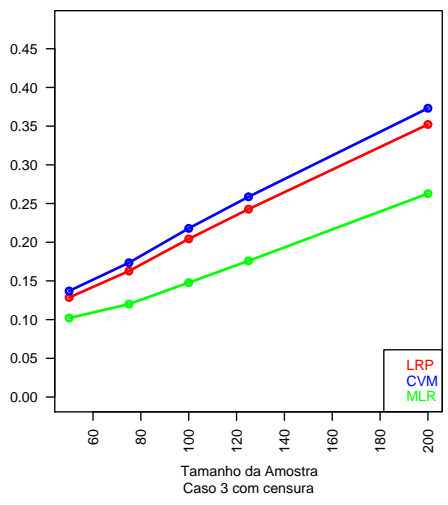

Peso: Gehan

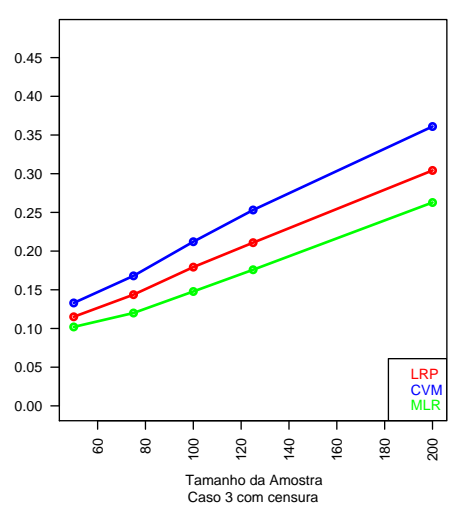

Peso: Peto e Peto

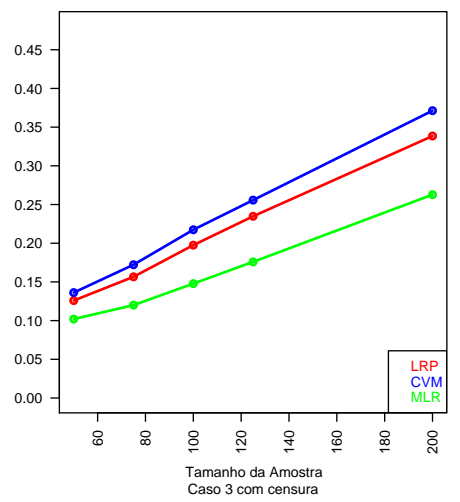

Peso: Tarone e Ware, Gamma $=0.5$

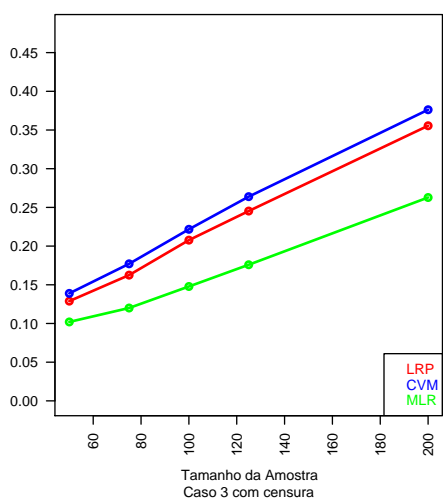

Peso: Peto e Peto Modificado

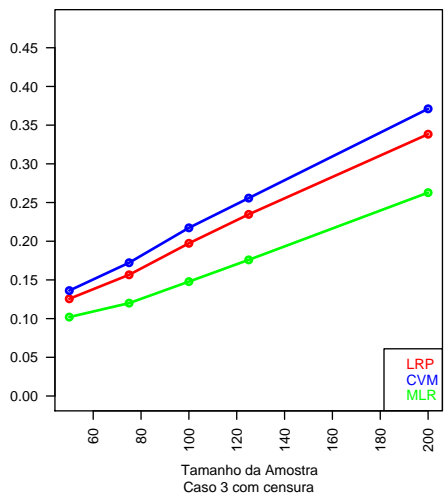

Figura 4.34: Tratamento versus Controle para o caso 3 com censura.

Na tabela (4.31) temos resultados do caso 3 na presença de censura. Notamos que em todos os processos de ponderação utilizados aa estatística de CVM e LRP são similares e ambas são mais poderosas em relação a MLR. 


\begin{tabular}{|c|c|c|c|c|c|c|c|c|c|c|c|c|}
\hline \multicolumn{13}{|c|}{ Caso 4 sem censura } \\
\hline TA & Peso & LRP & CVM & MLR & Peso & LRP & CVM & MLR & Peso & LRP & CVM & MLR \\
\hline 50 & Log-Rank & 0,3024 & 0,2603 & 0,1826 & Tarone & 0,2882 & 0,2134 & 0,1826 & Peto & 0,2763 & 0,1929 & 0,1826 \\
\hline 75 & & 0,4223 & 0,3641 & 0,2022 & e & 0,4058 & 0,2933 & 0,2022 & e & 0,3847 & 0,2642 & 0,2022 \\
\hline 100 & & 0,5304 & 0,4592 & 0,2301 & Ware & 0,5105 & 0,373 & 0,2301 & Peto & 0,4849 & 0,3369 & 0,2301 \\
\hline 125 & & 0,6224 & 0,5514 & 0,2923 & $\gamma=0,5$ & 0,6041 & 0,4505 & 0,2923 & & 0,5767 & 0,4049 & 0,2923 \\
\hline 200 & & 0,8168 & 0,7429 & 0,459 & & 0,7985 & 0,6361 & 0,459 & & 0,7695 & 0,5814 & 0,459 \\
\hline 50 & Gehan & 0,2681 & 0,1766 & 0,1826 & Fleming & 0,2679 & 0,1951 & 0,1826 & Peto & 0,2753 & 0,1919 & 0,1826 \\
\hline 75 & & 0,3732 & 0,2399 & 0,2022 & e & 0,3753 & 0,2637 & 0,2022 & e & 0,384 & 0,2635 & 0,2022 \\
\hline 100 & & 0,4737 & 0,3066 & 0,2301 & Harrington & 0,4722 & 0,3391 & 0,2301 & Peto & 0,4847 & 0,3364 & 0,2301 \\
\hline 125 & & 0,5639 & 0,3674 & 0,2923 & $\gamma=0,5 \mathbf{e} \rho=2$ & 0,564 & 0,4045 & 0,2923 & Modificado & 0,5763 & 0,404 & 0,2923 \\
\hline 200 & & 0,7564 & 0,5328 & 0,459 & & 0,7544 & 0,5797 & 0,459 & & 0,7692 & 0,5812 & 0,459 \\
\hline
\end{tabular}

Tabela 4.32: Tratamento versus Controle para o caso 4 sem censura.

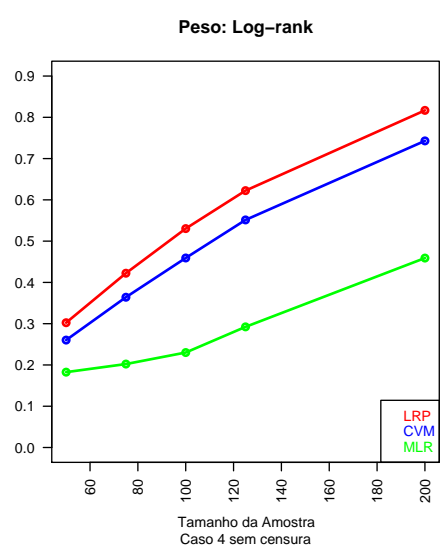

Peso: Fleming e Harrington, Gamma=0.5,Rho=2

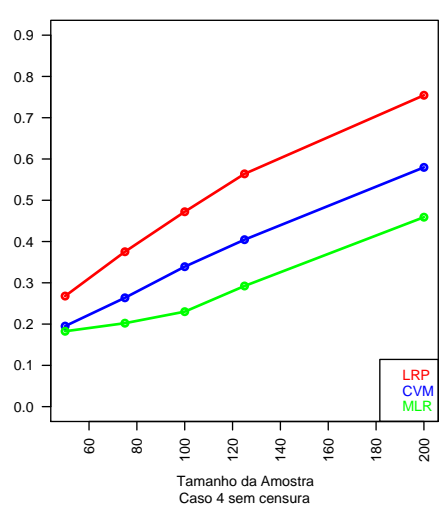

Peso: Gehan

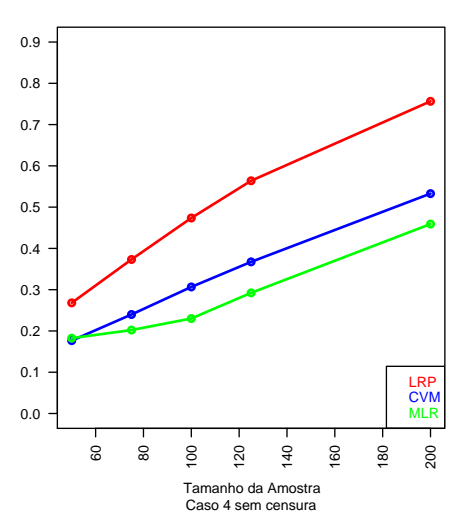

Peso: Peto e Peto

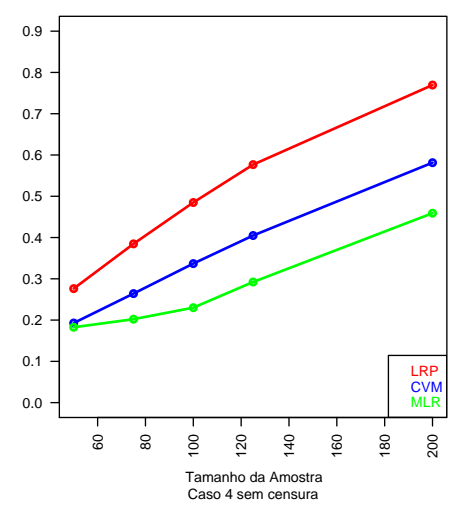

Peso: Tarone e Ware, Gamma=0.5

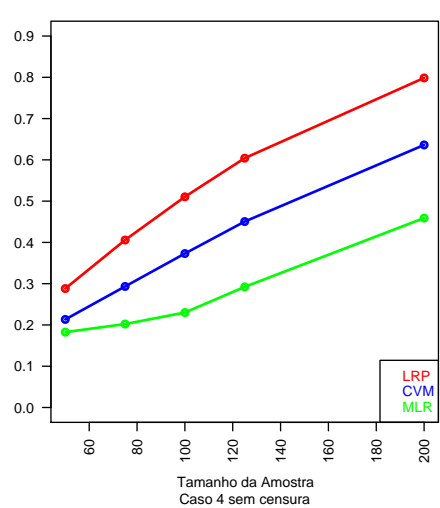

Peso: Peto e Peto Modificado

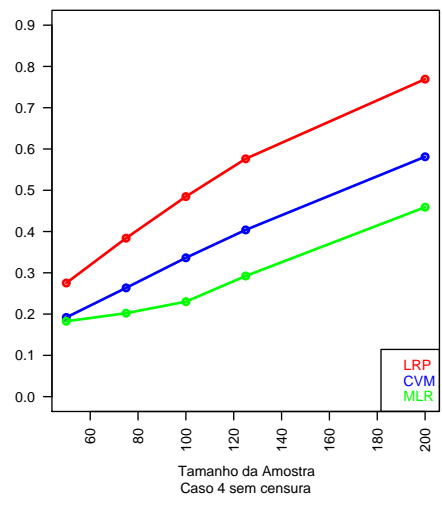

Figura 4.35: Tratamento versus Controle para o caso 4 sem censura.

Na tabela (4.32) temos resultados do caso 4 em que $W^{n_{1}}$ tem taxa constante $h^{n_{1}}(\ell)=0,15$ e $W^{n_{2}}$ tem taxa constante $h^{n_{2}}(\ell)=0,2$, para as duas taxas temos $\ell \in \mathcal{A}=\{1, \ldots, 10\} \subset \mathbb{N}$ na ausência de censura. Pelos resultados obtidos, notamos que em todos os processos de ponderação utilizados a estatística LRP é mais poderosa que as demais, exceto no processo de ponderação log-rank, em que LRP e CVM estão bem próximos. Como estamos no caso de riscos proporcionais, LRP é mais poderosa pois ela têm poder ótimo para estes tipos riscos das funções de intensidade (ver Klein e Moeschberger (1997)[15]). 


\begin{tabular}{|c|c|c|c|c|c|c|c|c|c|c|c|c|c|c|}
\hline \multirow[b]{2}{*}{ TA } & \multirow[b]{2}{*}{ Peso } & \multirow[b]{2}{*}{ LRP } & \multirow[b]{2}{*}{ CVM } & \multirow[b]{2}{*}{ MLR } & \multirow[b]{2}{*}{ Peso } & \multicolumn{3}{|c|}{ Caso 4 com censura } & \multirow[b]{2}{*}{ Peso } & \multirow[b]{2}{*}{ LRP } & \multirow[b]{2}{*}{ CVM } & \multirow[b]{2}{*}{ MLR } & \multirow[b]{2}{*}{$\%$ Cens1 } & \multirow[b]{2}{*}{$\%$ Cens 2} \\
\hline & & & & & & LRP & CVM & MLR & & & & & & \\
\hline 50 & Log-Rank & 0,1831 & 0,1919 & 0,1268 & Tarone & 0,1819 & 0,1762 & 0,1268 & Peto & 0,1798 & 0,1733 & 0,1268 & & \\
\hline 75 & & 0,2411 & 0,2573 & 0,1646 & e & 0,2398 & 0,2314 & 0,1646 & e & 0,2375 & 0,2249 & 0,1646 & & \\
\hline 100 & & 0,3091 & 0,3321 & 0,2009 & Ware & 0,3054 & 0,299 & 0,2009 & Peto & 0,3021 & 0,2931 & 0,2009 & 44,04 & 33,39 \\
\hline 125 & & 0,3645 & 0,3959 & 0,2455 & $\gamma=0,5$ & 0,363 & 0,3543 & 0,2455 & & 0,3592 & 0,3471 & 0,2455 & & \\
\hline \multirow[t]{2}{*}{200} & & 0,5211 & 0,5649 & 0,3706 & & & & & & & & & & \\
\hline & & & & & & 0,5186 & 0,5187 & 0,3706 & & 0,5158 & 0,5061 & 0,3706 & & \\
\hline 50 & Gehan & 0,1767 & 0,1606 & 0,1268 & Fleming & 0,1812 & 0,1755 & 0,1268 & Peto & 0,1797 & 0,1729 & 0,1268 & & \\
\hline 75 & & 0,234 & 0,2059 & 0,1646 & e & 0,2371 & 0,232 & 0,1646 & e & 0,2376 & 0,2246 & 0,1646 & & \\
\hline 100 & & 0,2974 & 0,2684 & 0,2009 & Harrington & 0,303 & 0,2978 & 0,2009 & Peto & 0,302 & 0,2929 & 0,2009 & 44,04 & 33,39 \\
\hline 125 & & 0,3527 & 0,314 & 0,2455 & $\gamma=0,5 \mathbf{e} \rho=2$ & 0,3606 & 0,3531 & 0,2455 & Modificado & 0,3592 & 0,3469 & 0,2455 & & \\
\hline 200 & & 0,5082 & 0,4634 & 0,3706 & & 0,5163 & 0,5156 & 0,3706 & & 0,5158 & 0,506 & 0,3706 & & \\
\hline
\end{tabular}

Tabela 4.33: Tratamento versus Controle para o caso 4 com censura.

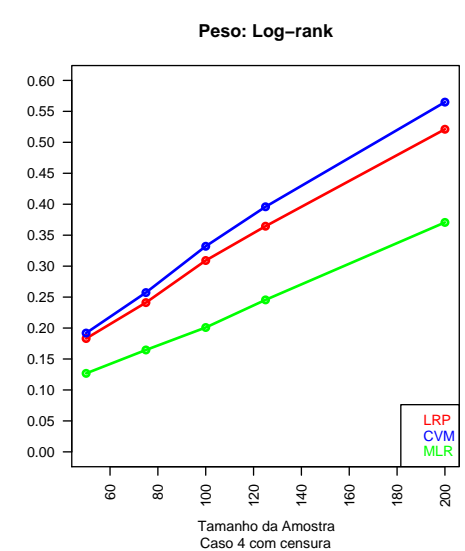

Peso: Fleming e Harrington, Gamma=0.5,Rho=2

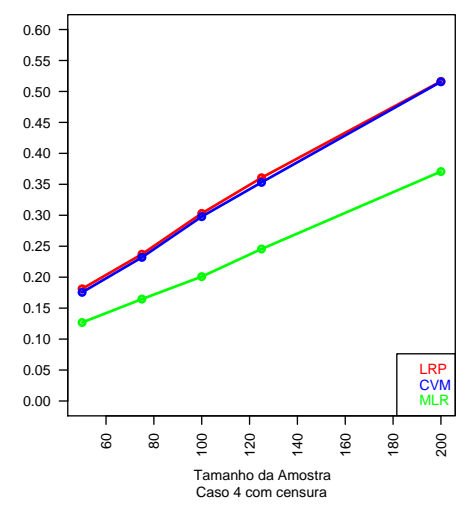

Peso: Gehan

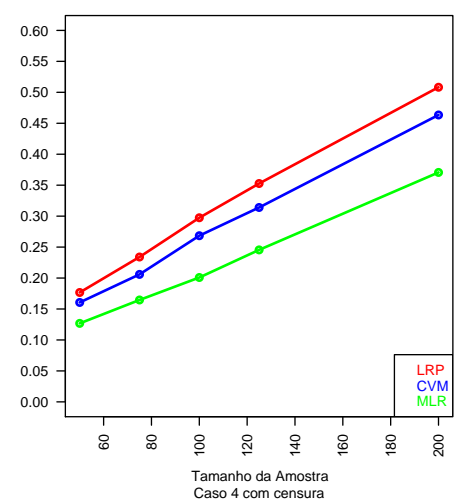

Peso: Peto e Peto

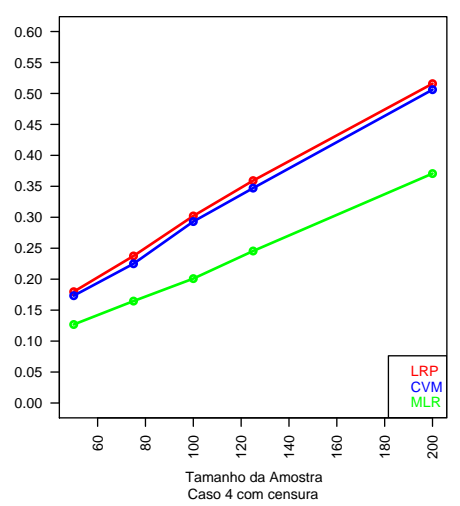

Peso: Tarone e Ware, Gamma=0.5

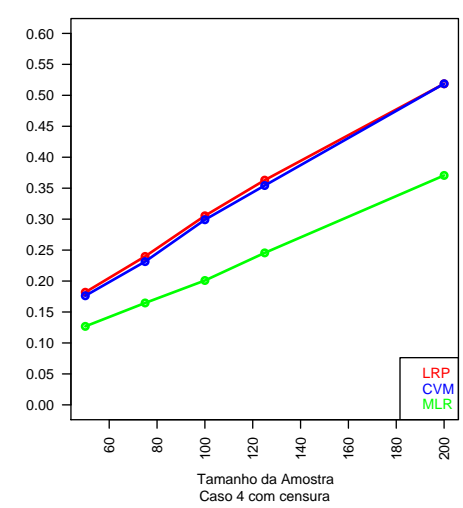

Peso: Peto e Peto Modificado

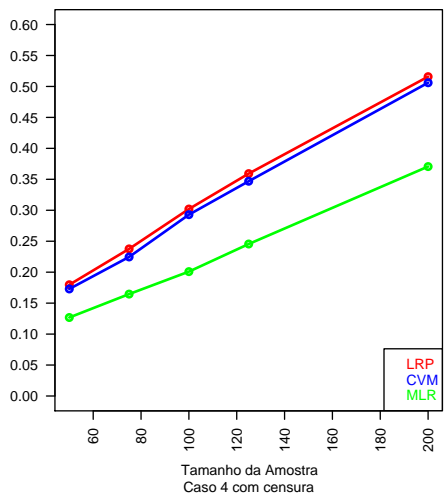

Figura 4.36: Tratamento versus Controle para o caso 4 com censura.

Na tabela (4.33) temos resultados do caso 4 na presença de censura. Notamos que em todos os processos de ponderação utilizados as estatísticas CVM e LRP são similares, e ambos são mais poderosos que MLR.

Um balanço geral dos casos estudados é que apenas o caso $4\left(h^{n_{1}}=0,15\right.$ e $\left.h^{n_{1}}=0,2\right)$ sem censura, a estatística LRP é mais poderosa que as demais. Nos demais casos, temos que CVM é mais poderosa que LRP e MLR. 


\subsection{Simulação em populações para dados truncados}

Os testes propostos neste trabalho têm como hipótese o fato das populações envolvidas terem distribuições discretas, porém, nesta seção avaliamos os testes para distribuições contínuas truncadas, e nela escolhemos as situações truncamento total, truncado com uma, duas casas depois da vírgula e o caso puramente contínuo. Assim, o objetivo é avaliar o comportamento das estatísticas de Cramér-von Mises (CVM) e Log-rank Ponderado (LRP) em comparação com as estatísticas de Cramér-von Mises para dados contínuos (CCVM) e o de Renyi citado na seção (4.2.3) na ausência e presença de censura. No livro de Klein and Moeschberger (1997)[15] para tratar dados com cruzamentos da função de intensidade, assim como a de Renyi, temos a estatística de CCVM para duas populações $(J=2)$ na presença de censura, que são apresentadas em duas versões. Nos experimentos desta seção, vamos utilizar a primeira versão apresentada em [15]. Para a construção da estatística, utilizamos respectivamente o estimador da função de intensidade acumulada $\hat{H}^{n_{p}}(i)$ e o estimador de variância de $\hat{H}^{n_{p}}(i)$ dadas por

$$
\hat{H}^{n_{p}}(i)=\sum_{\ell=1}^{i} \hat{h}^{n_{p}}(\ell), \text { e } \sigma_{n_{p}}^{2}(i)=\sum_{\ell=1}^{i} \frac{\Delta R^{n_{p}}(\ell)}{V^{n_{p}}(\ell)\left[1-V^{n_{p}}(\ell)\right]}, \quad 0 \leq i \leq k, \quad p=1,2 .
$$

Assim, o estimator da variância de $\hat{H}^{n_{1}}(i)-\hat{H}^{n_{2}}(i)$ é dada por

$$
\sigma^{2}(i)=\sigma_{n_{1}}^{2}(i)+\sigma_{n_{2}}^{2}(i)
$$

Com isso, chegamos a primeira versão da estatística de Cramér-von Mises para dados contínuos e duas populações

$$
Q_{1}=\left(\frac{1}{\sigma^{2}(k)}\right)^{2} \sum_{\ell=1}^{i}\left[\hat{H}^{n_{1}}(i)-\hat{H}^{n_{2}}(i)\right]\left[\sigma^{2}(i)-\sigma^{2}(i-1)\right], \quad 0 \leq i \leq k
$$

Quando a hipótese nula é verdadeira, para tamanhos de amostras grandes a distribuição de $Q_{1}$ é a mesma que o de $R_{1}=\int_{0}^{1}[B(x)]^{2} d x$, em que $B(x)$ é um processo do movimento Browniano. A função de sobrevivência de $R_{1}$ é encontrada na Tabela C.6 do Apêndice C do livro de Klein and Moeschberger (1997)[15].

Nesta seção avaliamos os testes nas situações de truncamento dos dados em dois experimentos. No primeiro experimento, tomamos $W^{n_{1}}$ com distribuição Weibull ${ }^{7}$ com parâmetros de forma $\delta=3,14 \mathrm{e}$ de escala $\alpha=1$, em seguida tomamos outra $W^{n_{2}}$ com distribuição Exponencial $^{8} \lambda=2$. No segundo experimento, tomamos $W^{n_{1}} \sim \operatorname{Weibull}(\delta=2, \alpha=100)$ e $W^{n_{2}} \sim \operatorname{Exponencial}(\lambda=0,01)$.

\footnotetext{
${ }^{7}$ A parametrização da distribuição Weibull usada encontra-se no Apêndice (A.4)

${ }^{8}$ A parametrização da distribuição Exponencial e o cálculo da função de intensidade encontra-se no Apêndice (A.3)
} 
Inicialmente, avaliamos $W^{n_{1}}$ e $W^{n_{2}}$ sob a hipótese nula $H_{0}$, em seguida, avaliamos o comportamento da função poder em relação aos tamanhos de amostra.O gráfico das funções de intensidade das duas amostras estão na figura (4.37).

\section{Taxas de Falha}

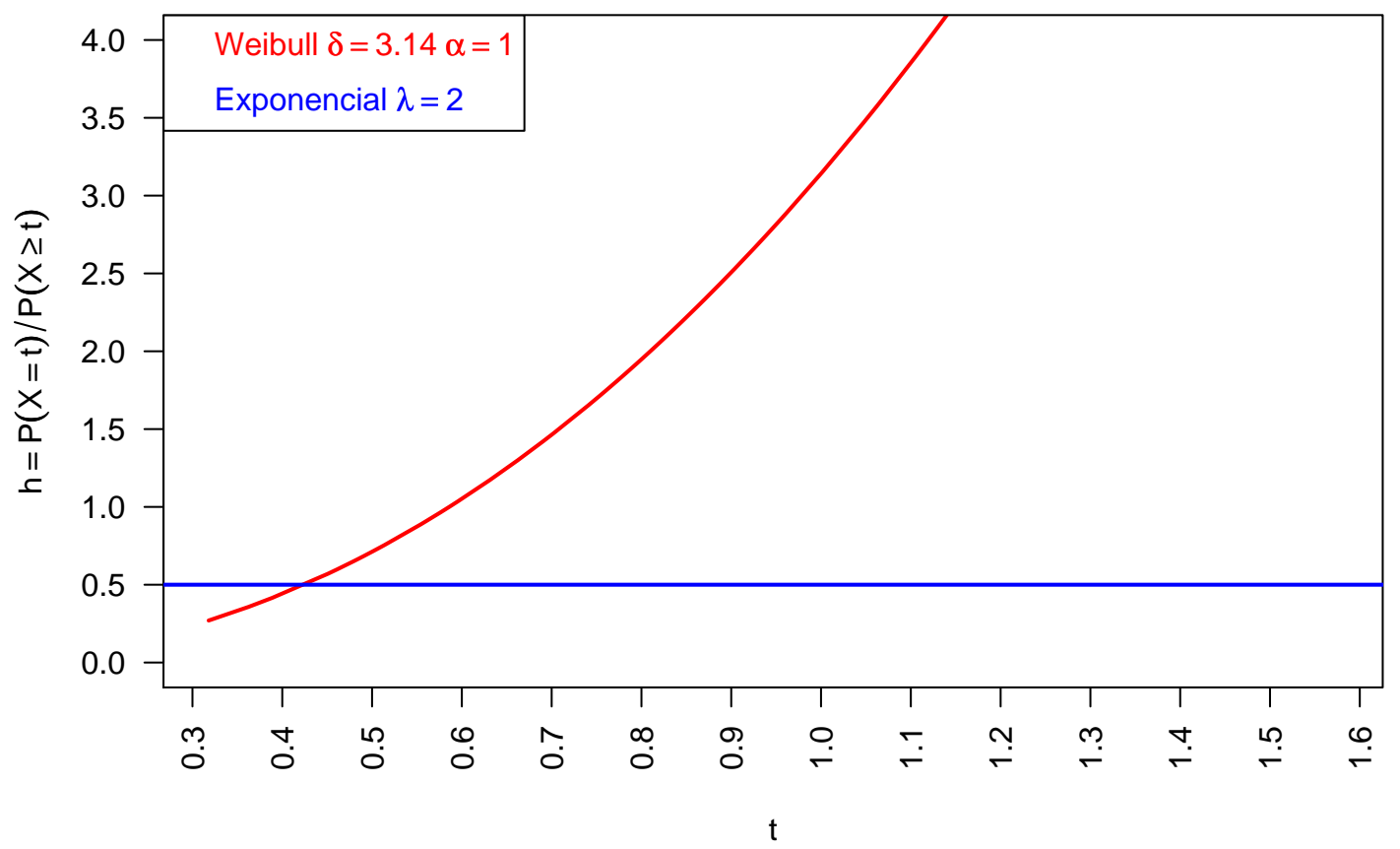

Figura 4.37: Taxa de falha das distribuições Weibull e Exponencial para o $1^{\circ}$ experimento.

Na primeira situação, sob a hipótese nula, vamos seguir o seguinte algoritmo:

1. Fixamos um nível de significância $\alpha=0,05$;

2. Geramos $W^{(1)}, W^{(2)}$ de tamanho TA com distribuição Exponencial com parâmetro $(\lambda=2)$ dado que a hipótese nula é verdadeira;

3. Escolhemos uma das funções de ponderação descrita na tabela (3.1);

4. No caso com censura, temos $C^{(1)}, C^{(2)} \sim \operatorname{Exp}(\lambda), \operatorname{com} \lambda=2$;

5. Realizamos o teste de hipótese usando valor crítico ao nível de significância $\alpha$;

6. Verificamos se cometeu o erro do tipo I, isto é, se a hipótese nula foi rejeitada;

$$
y_{m}= \begin{cases}1, & \text { se } H_{0} \text { é rejeitada ao nível de significância } \alpha \\ 0, & \text { caso contrário }\end{cases}
$$

7. Repetimos as etapas 2 a $4 m=10000$ vezes; 
8. Estimamos a probabilidade de cometer o erro do tipo I da seguinte forma:

$$
\hat{\alpha}=\frac{1}{m} \sum_{k=1}^{m} y_{k}
$$

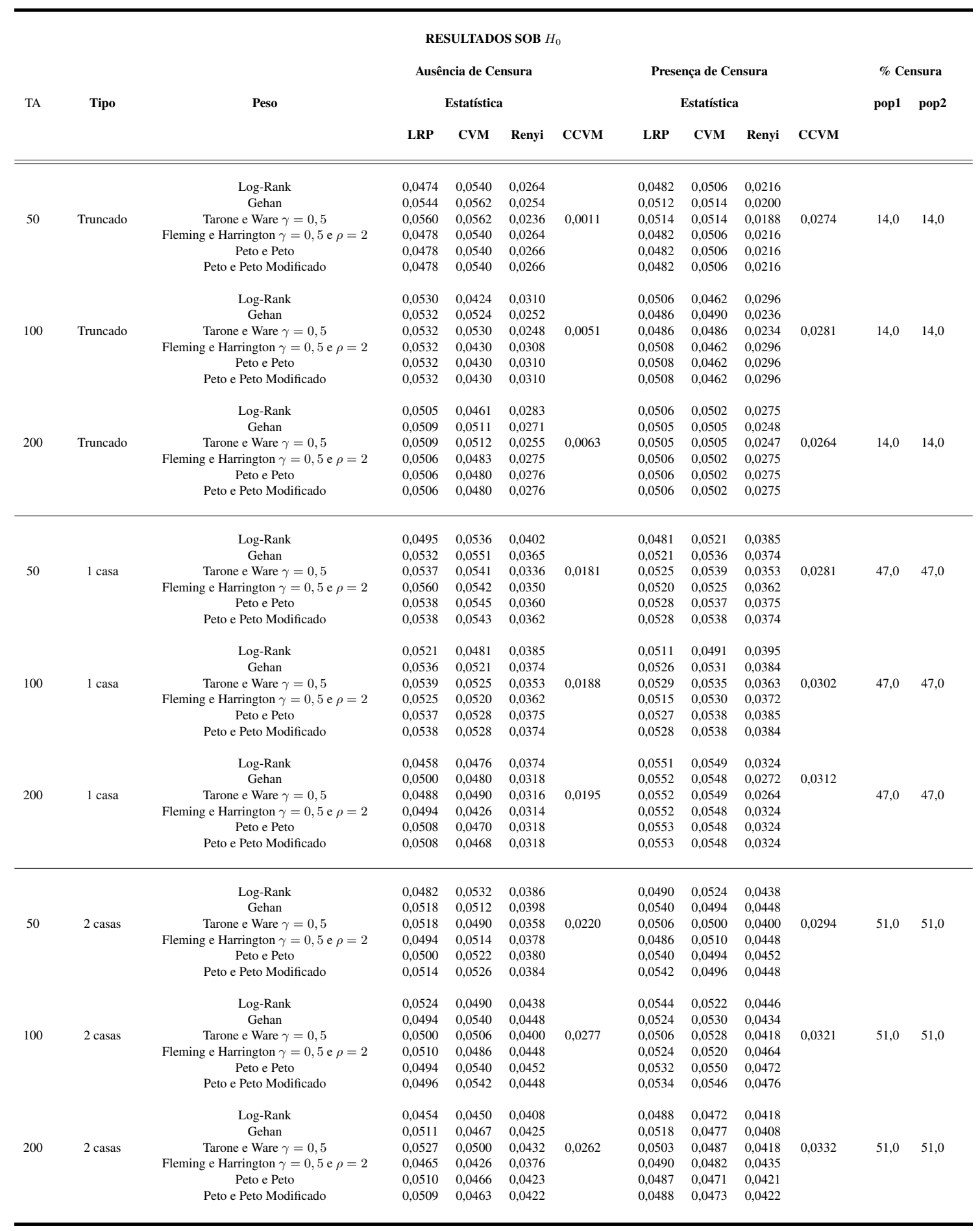

Tabela 4.34: Resultado sob $H_{0}$ para dados truncados e contínuos. 
Log-rank

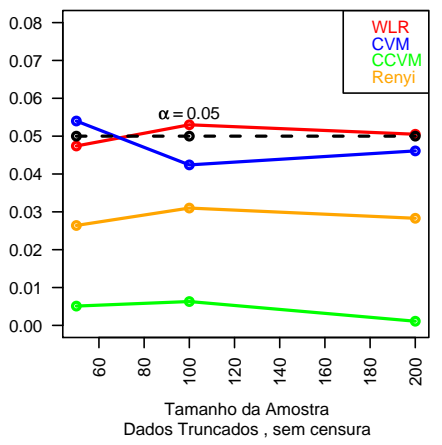

Fleming e Harrington $\gamma=0.5 \rho=2$

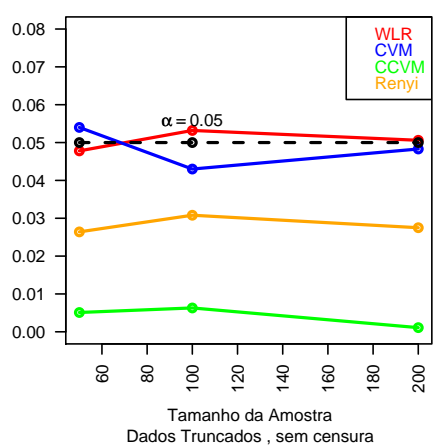

Gehan

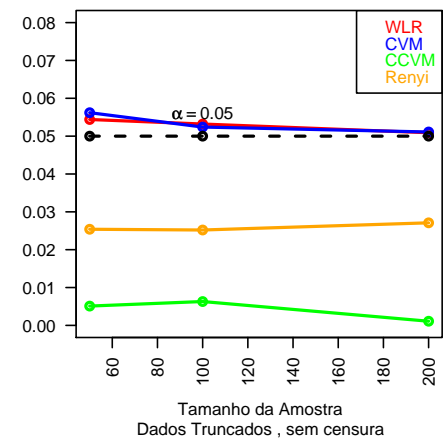

Peto e Peto

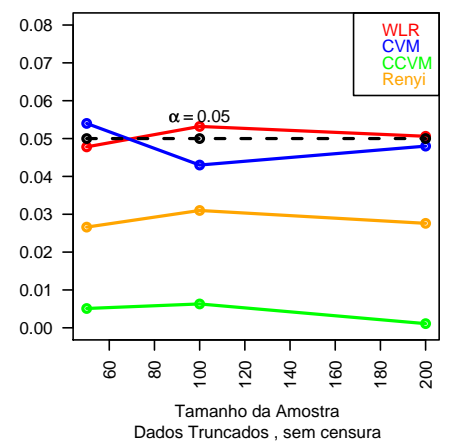

Tarone e Ware $\gamma=0.5$

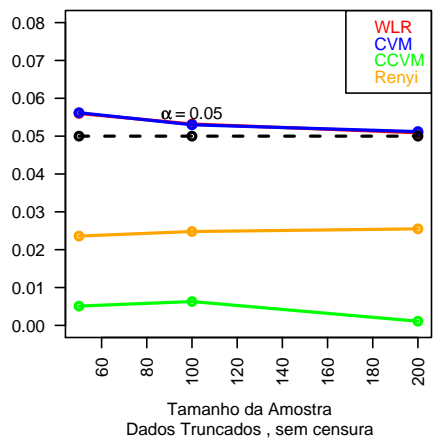

Peto e Peto Mod

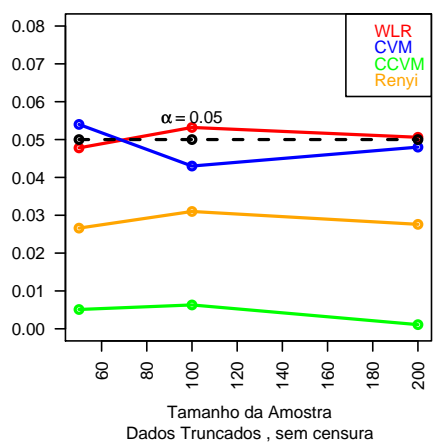

Figura 4.38: Gráfico da Simulação sob $H_{0}$ para dados truncados e sem censura.

Log-rank

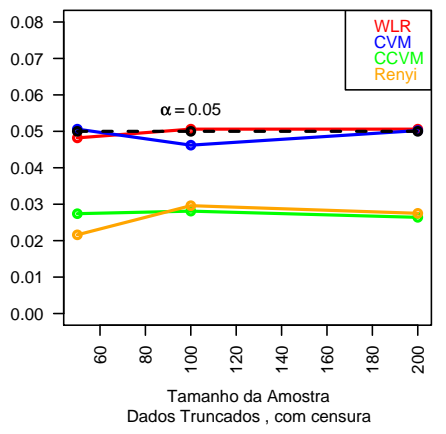

Fleming e Harrington $\gamma=0.5 \rho=2$

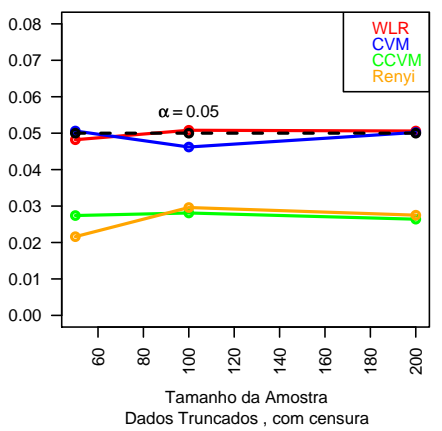

Gehan

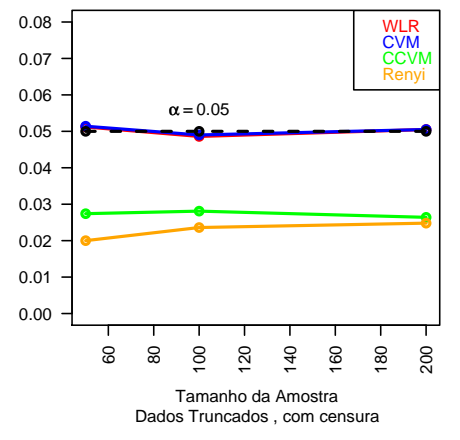

Peto e Peto

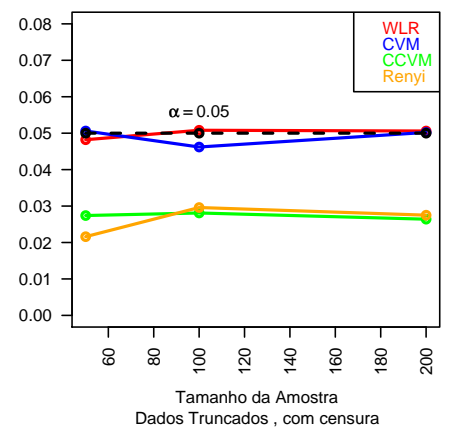

Tarone e Ware $\gamma=0.5$

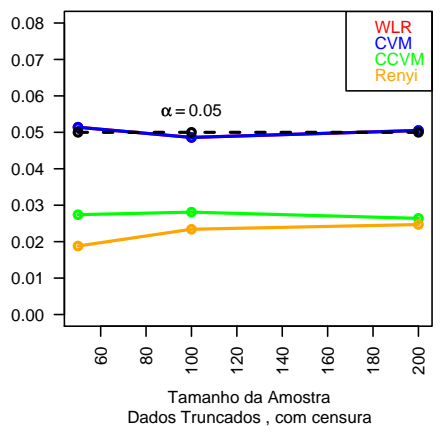

Peto e Peto Mod

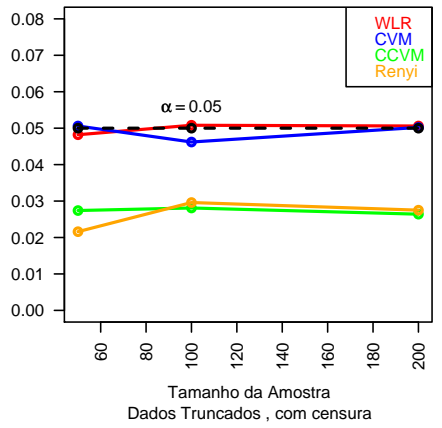

Figura 4.39: Gráfico da Simulação sob $H_{0}$ para dados truncados e com censura. 
Log-rank

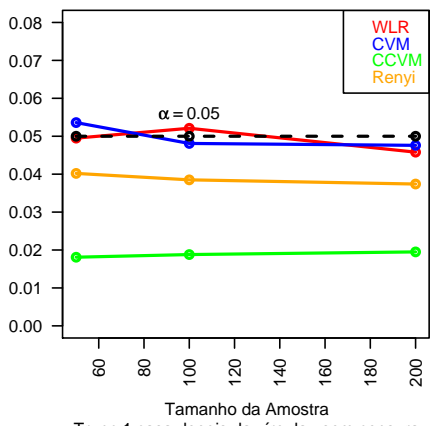

Trunc. 1 casa depois da vírgula, sem censura

Fleming e Harrington $\gamma=0.5 \rho=2$

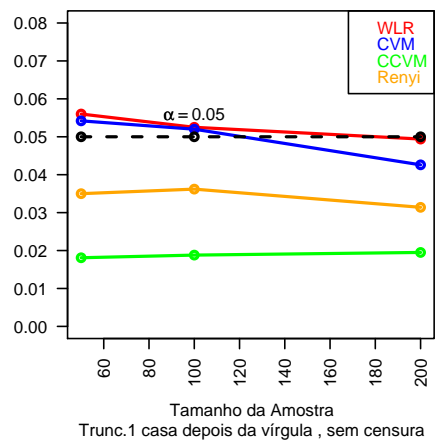

Gehan

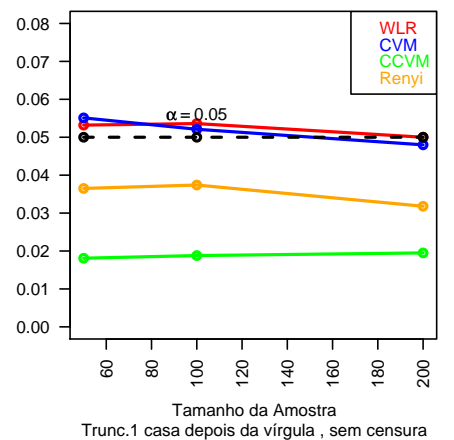

Peto e Peto

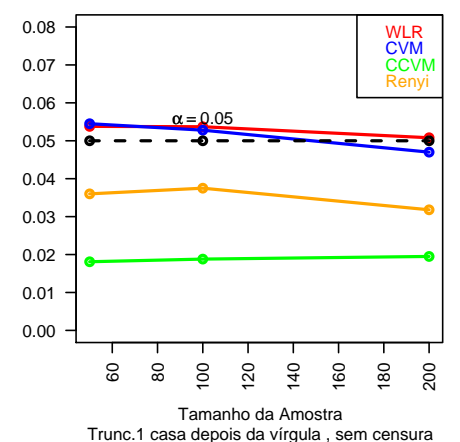

Tarone e Ware $\gamma=0.5$

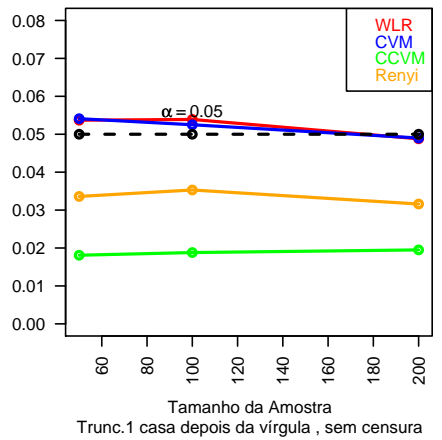

Peto e Peto Mod

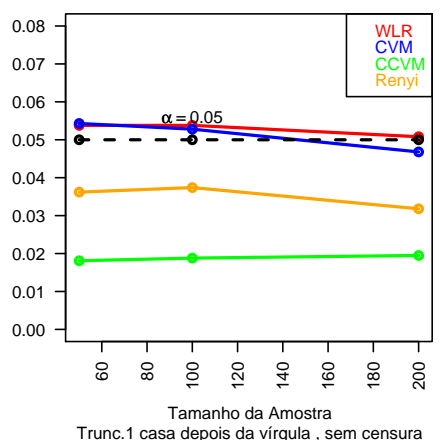

Figura 4.40: Gráfico da Simulação sob $H_{0}$ para dados truncados com 1 casa e sem censura.

Log-rank

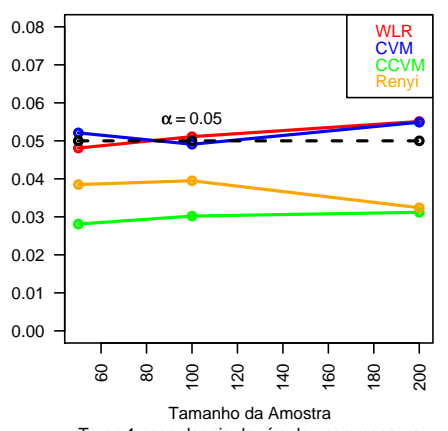

Trunc. 1 casa depois da vírgula , com censura

Fleming e Harrington $\gamma=0.5 \rho=2$

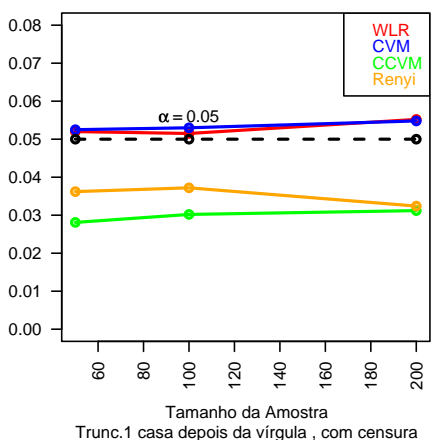

Gehan

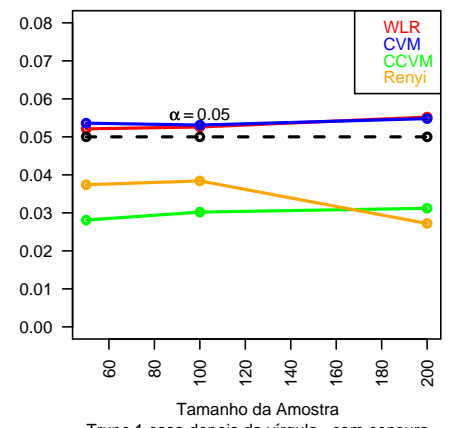

Peto e Peto

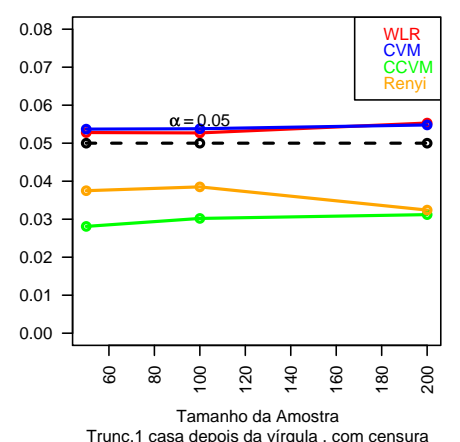

Tarone e Ware $\gamma=0.5$

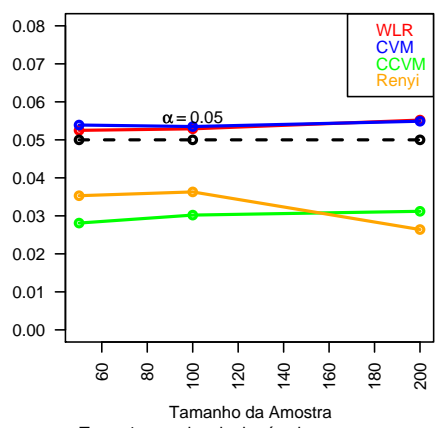

Trunc. 1 casa depois da virgula , com censura

Peto e Peto Mod

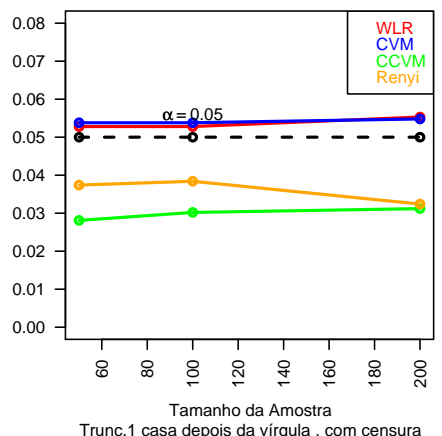

Figura 4.41: Gráfico da Simulação sob $H_{0}$ para dados truncados com 1 casa e com censura.

Assim como nos resultados obtidos para o caso discreto, a estatística de Renyi também não se aproxima do nível de significância nominal de 0,05 quando truncamos completamente os dados (“discretos"), com 
Log-rank

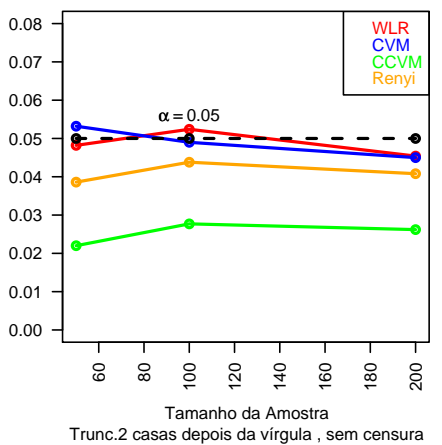

Fleming e Harrington $\gamma=0.5 \rho=2$

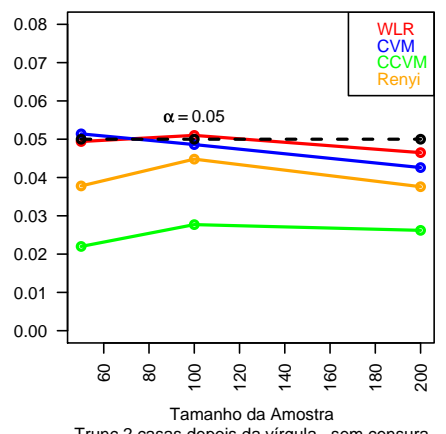

Gehan

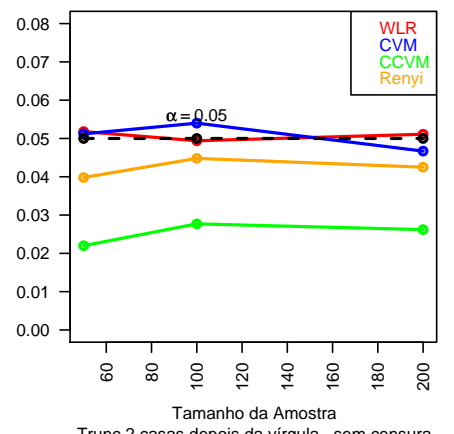

Peto e Peto

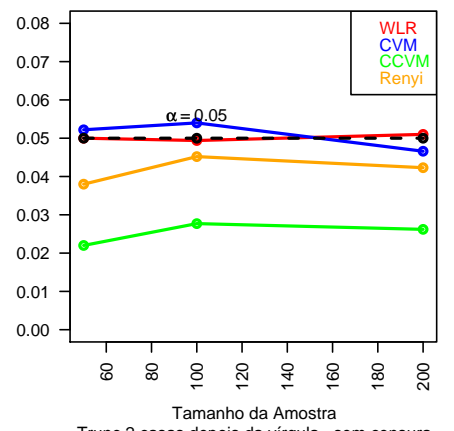

Tarone e Ware $\gamma=0.5$

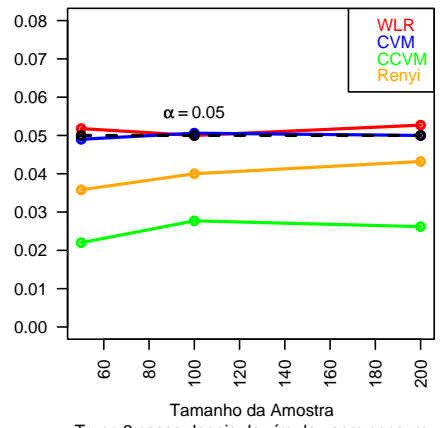

Tamanho da Amostra
Trunc. 2 casas depois da vírgula, sem censura

Peto e Peto Mod.

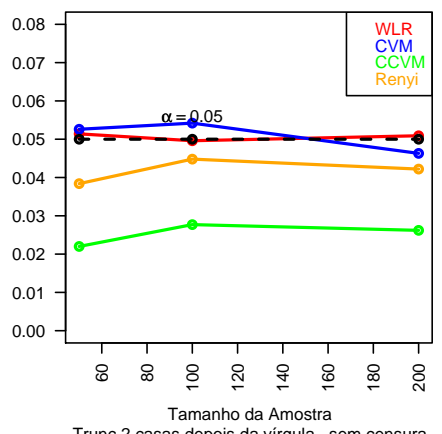

Tamanho da Amostra
Trunc.2 casas depois da virgula, sem censura

Figura 4.42: Gráfico da Simulação sob $H_{0}$ para dados truncados com 2 casas e sem censura.

Log-rank

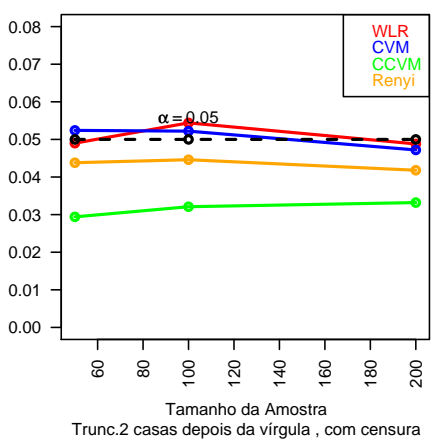

Fleming e Harrington $\gamma=0.5 \rho=2$

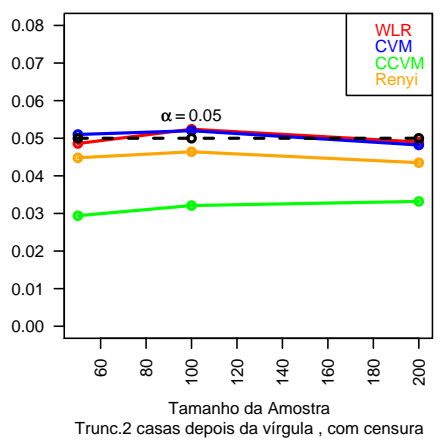

Gehan
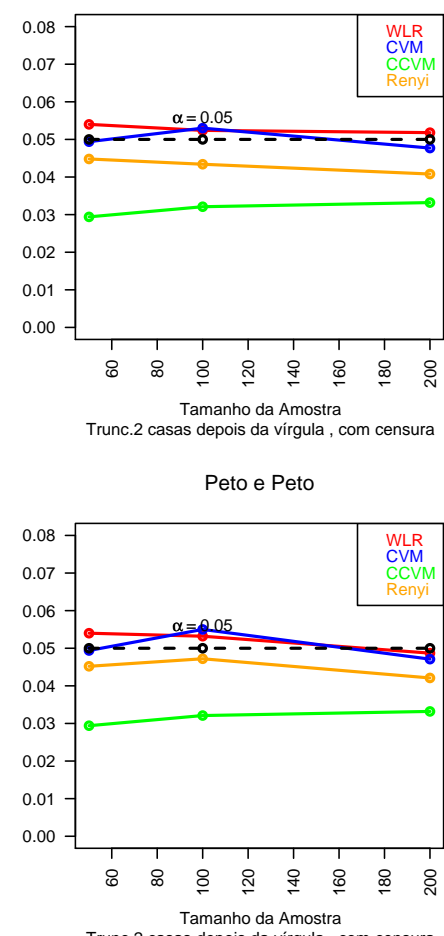

Trunc.2 casas depois da virgula, com censura
Tarone e Ware $\gamma=0.5$

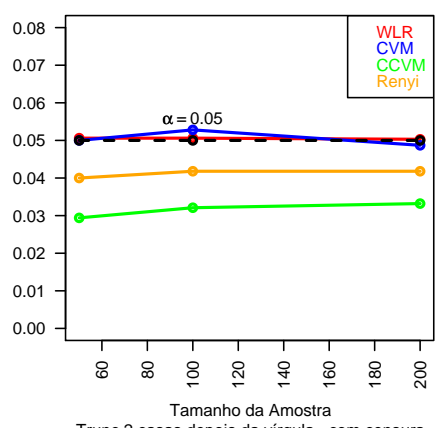

Peto e Peto Mod.

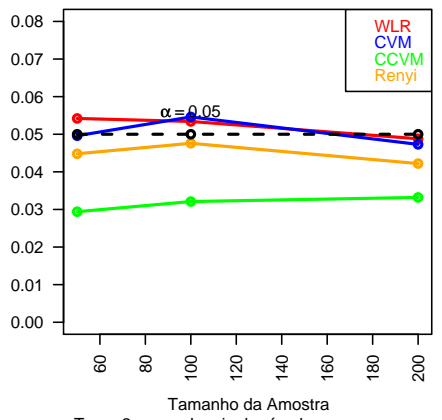

Tamanho da Amostra
Trunc. 2 casas depois da virgula , com censura

Figura 4.43: Gráfico da Simulação sob $H_{0}$ para dados truncados com 2 casas e com censura.

uma casa e com duas casas depois da vírgula. Observamos que a estatística de Cramér-von Mises para dados contínuos (CCVM), assim como a de Renyi, não se aproxima do nível de significância nominal de 
0,05 em nenhum dos três tipos de truncamento. Assim, não vamos comparar as estatísticas de Renyi e CCVM em nenhum dos casos. Ao contrário das estatísticas Cramér-von Mises e Log-rank Ponderado, que estão muito próximas da probabilidade nominal $\alpha=0,05$, tanto na ausência como na presença de censura dos dados.

A seguir, para estudar o comportamento do problema de cruzamento das funções de intensidade, comparamos o teste de Log-rank Ponderado (LRP) e o de Cramér-von Mises (CVM) para dados discretos, ambos na presença e ausência de censura arbitrária nos casos de truncamento completo, com uma casa e duas casas depois da vírgula dos dados contínuos.

Neste primeiro experimento, vamos seguir o seguinte algoritmo.

1. Fixamos um nível de significância $\alpha=0,05$;

2. Escolhemos uma das funções de ponderação descrita na tabela (3.1);

3. Geramos populações de tamanho da amostra TA e definimos duas populações com distribuições:

- $W^{(1)} \sim \operatorname{Wei}(\delta=3,14 ; \alpha=1)$

- $W^{(2)} \sim \operatorname{Exp}(\lambda=2)$ dado que a hipótese nula é falsa;

4. Para o caso com censura, temos $C^{(1)}, C^{(2)} \sim \operatorname{Exp}(\lambda=0,15)$ truncado com o número de casas do experimento;

5. Realizamos o teste de hipótese usando valor crítico ao nível de significância $\alpha$;

6. Para estimarmos a função poder tomamos:

$$
y_{m}=\left\{\begin{array}{cc}
1, & \text { se } H_{0} \text { foi rejeitada ao nível de significância } \alpha \\
0, & \text { caso contrário }
\end{array}\right.
$$

7. Repetimos as etapas 2 a $4=10000$ vezes;

8. Estimamos o poder do teste da seguinte forma:

$$
\text { Poder }=\frac{1}{m} \sum_{k=1}^{m} y_{k}
$$




\begin{tabular}{|c|c|c|c|c|c|c|c|c|c|}
\hline \multicolumn{10}{|c|}{ Caso Truncado sem censura } \\
\hline $\mathbf{T A}$ & Peso & CVM & LRP & Peso & CVM & LRP & Peso & CVM & LRP \\
\hline 10 & & 0,3404 & 0,3353 & & 0,3425 & 0,3423 & & 0,3405 & 0,3354 \\
\hline 15 & & 0,4452 & 0,4321 & & 0,3475 & 0,3472 & & 0,4453 & 0,4322 \\
\hline 20 & Log-rank & 0,5414 & 0,5251 & Tarone e Ware & 0,4441 & 0,4442 & Peto e Peto & 0,5415 & 0,5251 \\
\hline 30 & & 0,5865 & 0,5725 & $\gamma=0,5$ & 0,5954 & 0,5951 & & 0,5866 & 0,5726 \\
\hline 40 & & 0,6922 & 0,6864 & & 0,6968 & 0,6967 & & 0,6921 & 0,6865 \\
\hline 45 & & 0,7501 & 0,7438 & & 0,7436 & 0,7433 & & 0,7502 & 0,7439 \\
\hline 10 & & 0,3404 & 0,3404 & & 0,3404 & 0,3353 & & 0,3404 & 0,3353 \\
\hline 15 & & 0,4453 & 0,4454 & & 0,4452 & 0,4321 & & 0,4455 & 0,4323 \\
\hline 20 & Gehan & 0,5417 & 0,5277 & Fleming e Harrington & 0,5414 & 0,5251 & Peto e Peto Modificado & 0,5418 & 0,5283 \\
\hline 30 & & 0,5869 & 0,5872 & $\gamma=0,5$ e $\rho=2$ & 0,5865 & 0,5725 & & 0,5869 & 0,5731 \\
\hline 40 & & 0,6925 & 0,6927 & & 0,692 & 0,6864 & & 0,6925 & 0,6863 \\
\hline 45 & & 0,7505 & 0,7506 & & 0,7501 & 0,7438 & & 0,7512 & 0,7439 \\
\hline
\end{tabular}

Tabela 4.35: Dados com truncamento completo na ausência de censura.

Log-rank

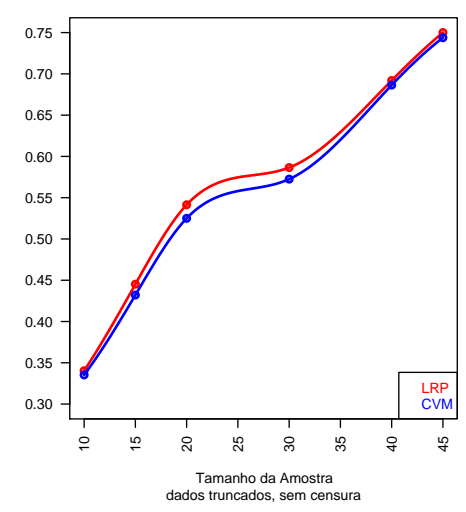

Fleming e Harrington (Gamma=0.5, rho=2)

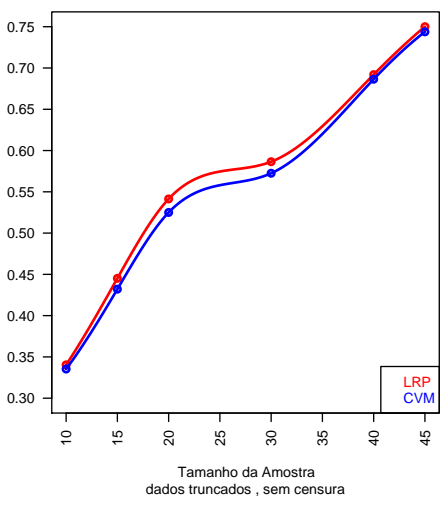

Gehan

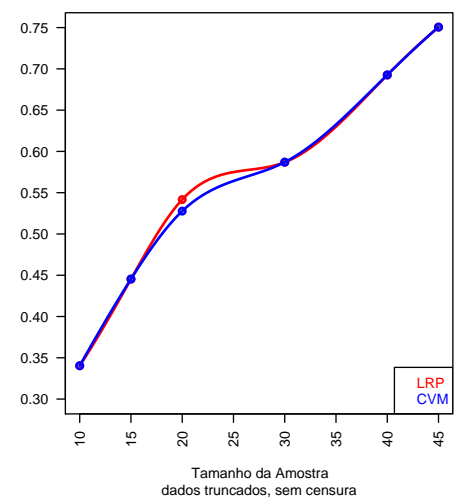

Peto e Peto

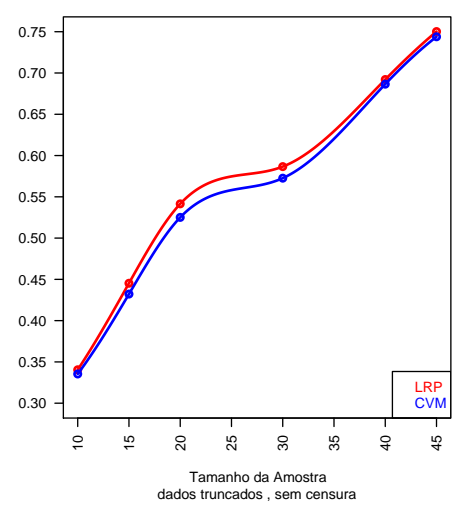

Tarone e Ware (Gamma=0.5)

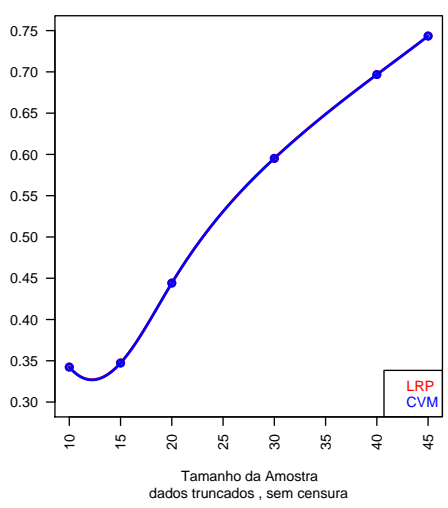

Peto e Peto Modificado

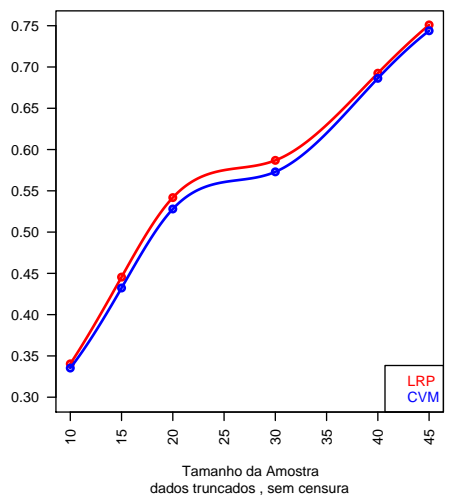

Figura 4.44: Gráfico para dados com truncamento completo na ausência de censura.

Nesta situação, estamos no caso de truncamento completo dos dados e ausência de censura. Note que, ambos têm um comportamento similar, fato devido a um cruzamento não tão severo, como no caso de cruzamento no meio da função de intensidade como mostrado na seção (4.2.2). Assim, ambos têm um bom comportamento para dados completamente truncados (“discretos”) na ausência de censura dos dados. 


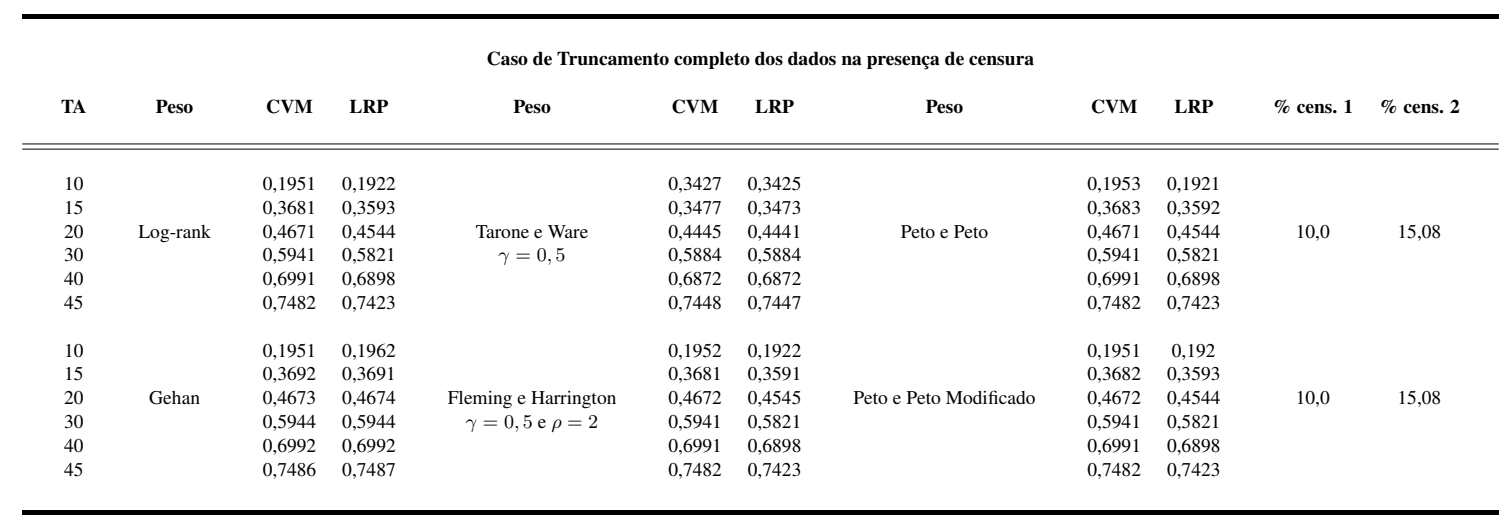

Tabela 4.36: Dados com truncamento completo na presença de censura.

Log-rank

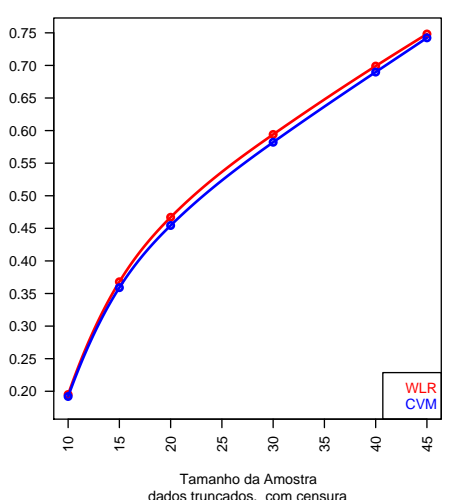

Fleming e Harrington (Gamma $=0.5$, Rho $=2$ )

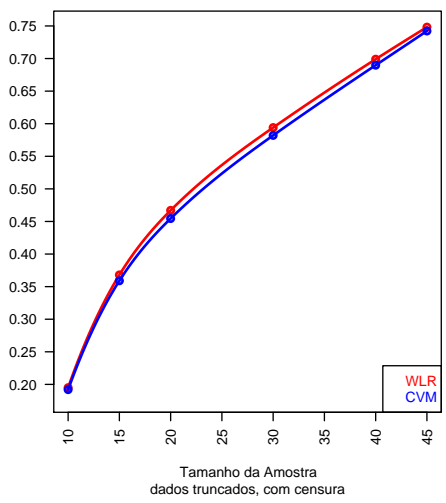

Gehan

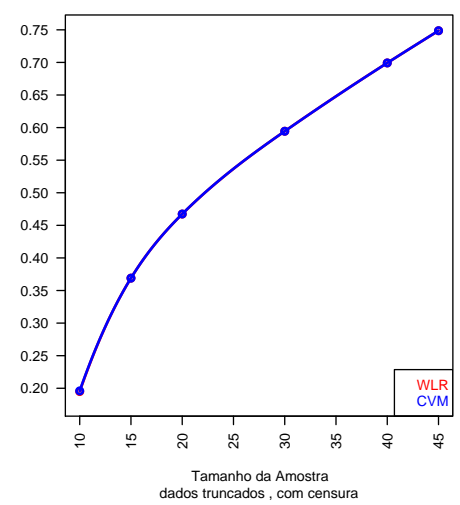

Peto e Peto

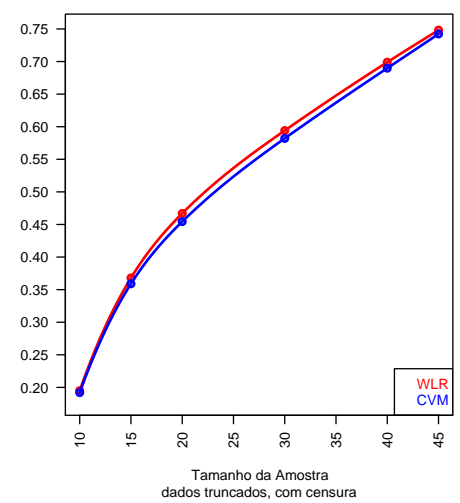

Tarone e Ware (Gamma=0.5)

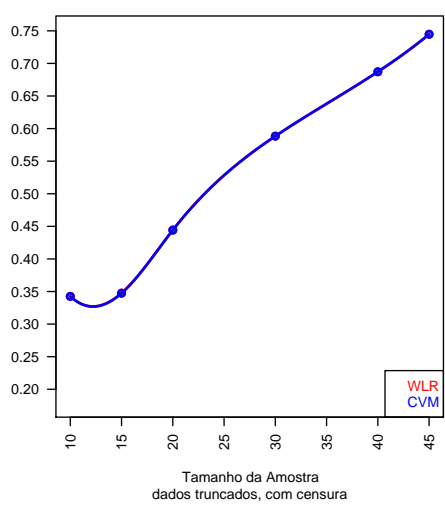

Peto e Peto Modificado

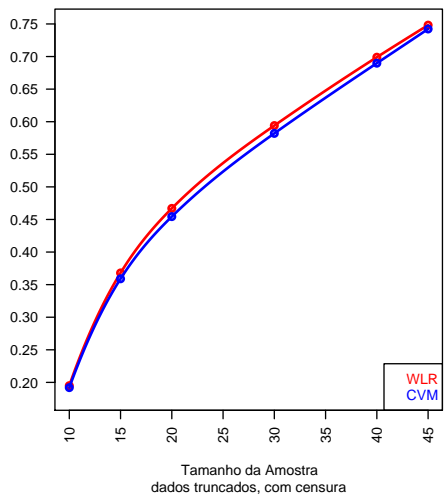

Figura 4.45: Gráfico para dados com truncamento completo na presença de censura.

Agora, estamos no caso de truncamento completo dos dados na presença de censura e como no caso em que não censuramos os dados, ambos têm um comportamento similar, fato devido a um cruzamento não tão severo. Logo, para dados com truncamento completo dos dados as duas estatísticas têm um bom comportamento tanto na presença como na ausência de censura dos dados. 


\begin{tabular}{|c|c|c|c|c|c|c|c|c|c|}
\hline \multicolumn{10}{|c|}{ Caso Truncado 1 casa na ausência de censura } \\
\hline TA & Peso & CVM & LRP & Peso & CVM & LRP & Peso & CVM & LRP \\
\hline 10 & & 0,5412 & 0,2501 & & 0,6266 & 0,3547 & & 0,6587 & 0,4195 \\
\hline 15 & & 0,6740 & 0,2831 & & 0,7864 & 0,4409 & & 0,8255 & 0,5485 \\
\hline 20 & Log-Rank & 0,7800 & 0,3065 & Tarone e Ware $\gamma=0,5$ & 0,8846 & 0,5131 & Peto e Peto & 0,9160 & 0,6563 \\
\hline 30 & & 0,9057 & 0,3418 & & 0,9685 & 0,6446 & & 0,9830 & 0,8147 \\
\hline 40 & & 0,9696 & 0,3897 & & 0,9923 & 0,7558 & & 0,9972 & 0,9069 \\
\hline 45 & & 0,9816 & 0,4189 & & 0,9968 & 0,8046 & & 0,9993 & 0,9384 \\
\hline 10 & & 0,6665 & 0,4424 & & 0,6080 & 0,3868 & & 0,6623 & 0,4275 \\
\hline 15 & & 0,8322 & 0,5690 & & 0,7461 & 0,4781 & & 0,8281 & 0,5580 \\
\hline 20 & Gehan & 0,9207 & 0,6765 & Fleming e Harrington $\gamma=0,5$ e $\rho=2$ & 0,8412 & 0,5633 & Peto e Peto Modificado & 0,9179 & 0,6661 \\
\hline 30 & & 0,9840 & 0,8257 & & 0,9420 & 0,6984 & & 0,9831 & 0,8197 \\
\hline 40 & & 0,9972 & 0,9143 & & 0,9816 & 0,8091 & & 0,9972 & 0,9106 \\
\hline 45 & & 0,9994 & 0,9437 & & 0,9898 & 0,8540 & & 0,9993 & 0,9407 \\
\hline
\end{tabular}

Tabela 4.37: Dados truncados com 1 casa na ausência de censura.
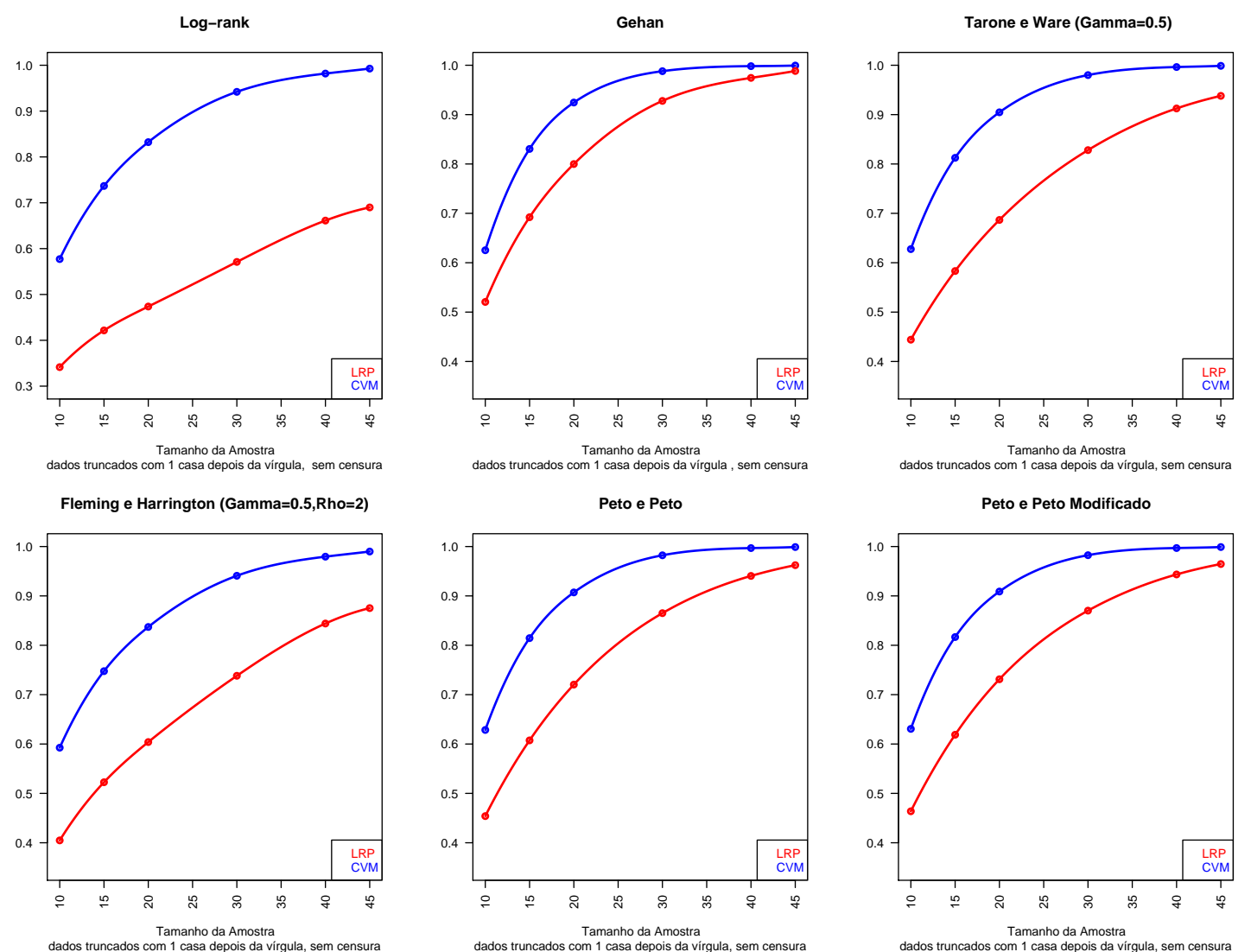

Figura 4.46: Gráfico para dados truncados com 1 casa na ausência de censura.

Agora, estamos no caso em que truncamos os dados com uma casa depois da vírgula e ausência de censura. Note que, a estatística de Cramér-von Mises têm função poder maior com destaque para o peso $\log$-rank $\left(u\left(n^{\star}, \ell\right)=1\right)$, em que a estatística de Log-rank Ponderado têm poder muito baixo para tamanhos de amostra até 45 . 


\begin{tabular}{|c|c|c|c|c|c|c|c|c|c|c|c|}
\hline \multicolumn{12}{|c|}{ Caso Truncado com 1 casa na presença de censura } \\
\hline TA & Peso & CVM & LRP & Peso & CVM & LRP & Peso & CVM & LRP & $\%$ cens 1 & $\%$ cens 2 \\
\hline 10 & & 0,5772 & 0,3415 & & 0,6276 & 0,4441 & & 0,6285 & 0,4540 & & \\
\hline 15 & & 0,7367 & 0,4216 & & 0,8125 & 0,5832 & & 0,8145 & 0,6074 & & \\
\hline 20 & Log-Rank & 0,8323 & 0,4737 & Tarone e Ware $\gamma=0,5$ & 0,9047 & 0,6867 & Peto e Peto & 0,9071 & 0,7204 & 23,4 & 33,41 \\
\hline 30 & & 0,9420 & 0,5710 & & 0,9801 & 0,8281 & & 0,9823 & 0,8651 & & \\
\hline 40 & & 0,9819 & 0,6612 & & 0,9964 & 0,9125 & & 0,9969 & 0,9405 & & \\
\hline 45 & & 0,9927 & 0,6899 & & 0,9988 & 0,9380 & & 0,9990 & 0,9623 & & \\
\hline 10 & & 0,6253 & 0,5206 & & 0,5926 & 0,4048 & & 0,6307 & 0,4638 & & \\
\hline 15 & & 0,8305 & 0,6922 & & 0,7476 & 0,5226 & & 0,8167 & 0,6188 & & \\
\hline 20 & Gehan & 0,9245 & 0,7999 & Fleming e Harrington $\gamma=0,5$ e $\rho=2$ & 0,8371 & 0,6040 & Peto e Peto Modificado & 0,9089 & 0,7312 & 23,4 & 33,41 \\
\hline 30 & & 0,9880 & 0,9278 & & 0,9408 & 0,7382 & & 0,9825 & 0,8702 & & \\
\hline 40 & & 0,9982 & 0,9744 & & 0,9795 & 0,8441 & & 0,9970 & 0,9435 & & \\
\hline 45 & & 0,9994 & 0,9884 & & 0,9899 & 0,8753 & & 0,9990 & 0,9646 & & \\
\hline
\end{tabular}

Tabela 4.38: Dados truncados com 1 casa na presença de censura.

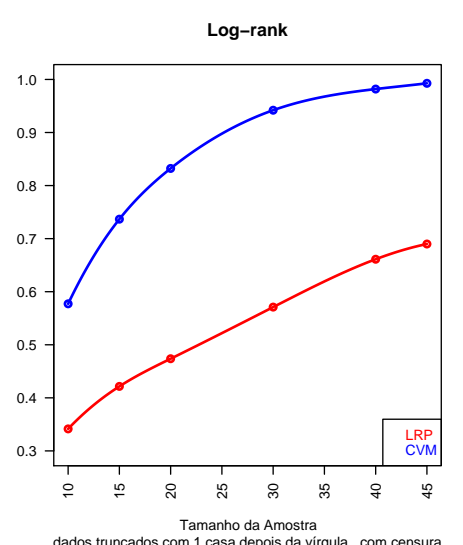

dados truncados com 1 casa depois da virgula, com censura

Fleming e Harrington (Gamma=0.5,Rho=2)

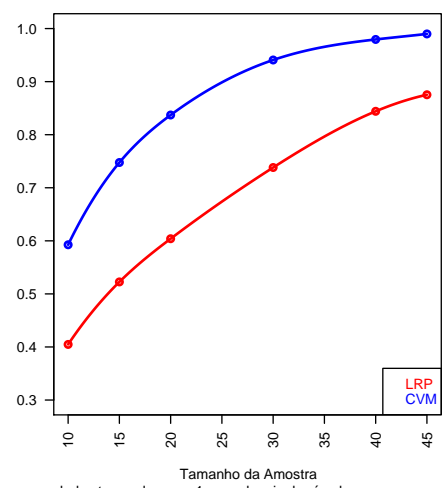

Tamanho da Amostra
dados truncados com 1 casa depois da virgula, com censura
Gehan

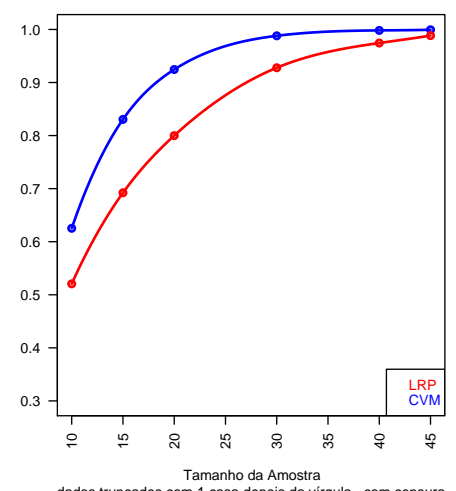

Peto e Peto

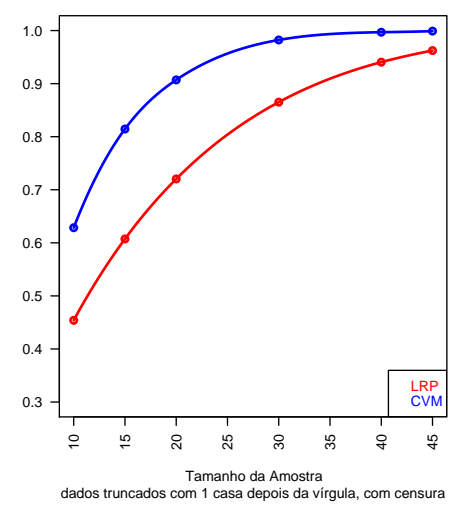

Tarone e Ware (Gamma=2)

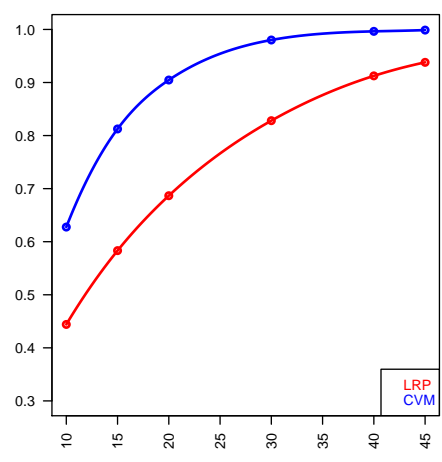

Tamanho da Amostra
dados truncados com 1 casa depois da virgula, com censura

Peto e Peto Modified

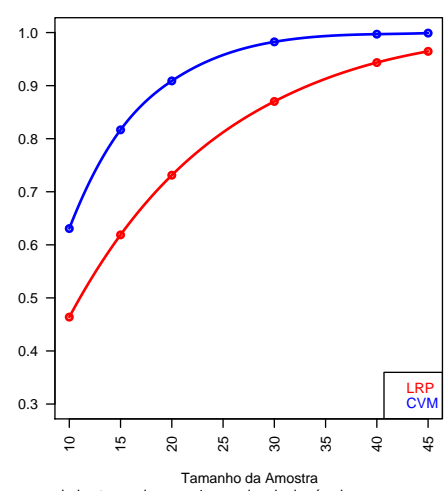

Tamanho da Amostra
dados truncados com 1 casa depois da virgula, com censura

Figura 4.47: Gráfico para dados truncados com 1 casa na presença de censura.

Agora, estamos no caso em que truncamos os dados com uma casa depois da vírgula e presença de censura. Note que, temos um comportamento similar ao caso sem censura. 


\begin{tabular}{|c|c|c|c|c|c|c|c|c|c|}
\hline \multicolumn{10}{|c|}{ Caso Truncado com 2 casas sem censura } \\
\hline TA & Peso & CVM & LRP & Peso & CVM & LRP & Peso & CVM & LRP \\
\hline 10 & & 0,7734 & 0,4828 & & 0,8278 & 0,6095 & & 0,8416 & 0,6868 \\
\hline 15 & & 0,9041 & 0,5833 & & 0,9504 & 0,7659 & & 0,9598 & 0,8533 \\
\hline 20 & Log-Rank & 0,9641 & 0,6653 & Tarone e Ware & 0,9863 & 0,8647 & Peto e Peto & 0,9912 & 0,936 \\
\hline 30 & & 0,9955 & 0,7901 & $\gamma=0,5$ & 0,9995 & 0,9552 & & 0,9999 & 0,9895 \\
\hline 40 & & 0,9996 & 0,8603 & & 1 & 0,9877 & & 1 & 0,9983 \\
\hline 45 & & 1 & 0,8983 & & 1 & 0,9924 & & 1 & 0,9999 \\
\hline 10 & & 0,8426 & 0,7032 & & 0,8141 & 0,6553 & & 0,8420 & 0,6951 \\
\hline 15 & & 0,9606 & 0,8625 & & 0,9350 & 0,8087 & & 0,9605 & 0,8600 \\
\hline 20 & Gehan & 0,9914 & 0,9404 & Fleming e Harrington & 0,9806 & 0,9000 & Peto e Peto Modificado & 0,9913 & 0,9393 \\
\hline 30 & & 0,9999 & 0,9902 & $\gamma=0,5$ e $\rho=2$ & 0,9987 & 0,9762 & & 0,9999 & 0,9902 \\
\hline 40 & & 1 & 0,9983 & & 0,9998 & 0,9943 & & 1 & 0,9983 \\
\hline 45 & & 1 & 0,9999 & & 1 & 0,9979 & & 1 & 0,9999 \\
\hline
\end{tabular}

Tabela 4.39: Dados truncados com 2 casas na ausência de censura.

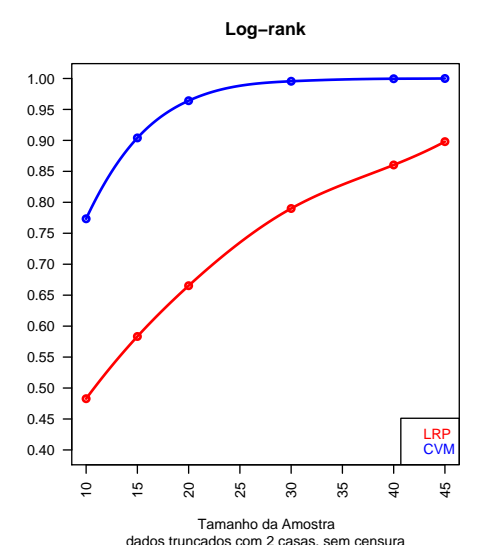

dados truncados com 2 casas, sem censura

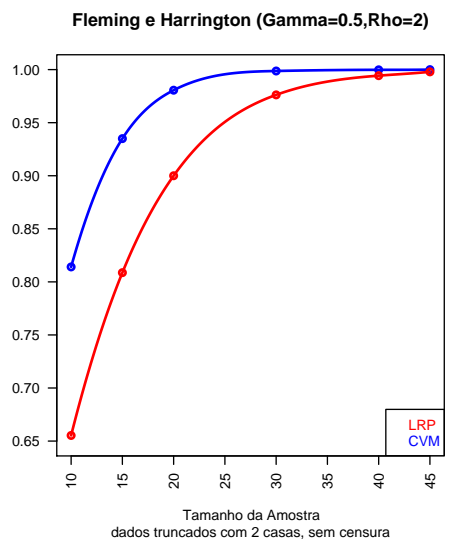

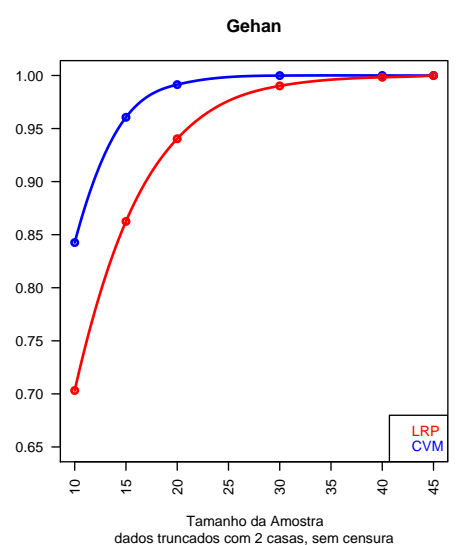

Tamanho da Amostra
dados truncados com 2 casas, sem censura

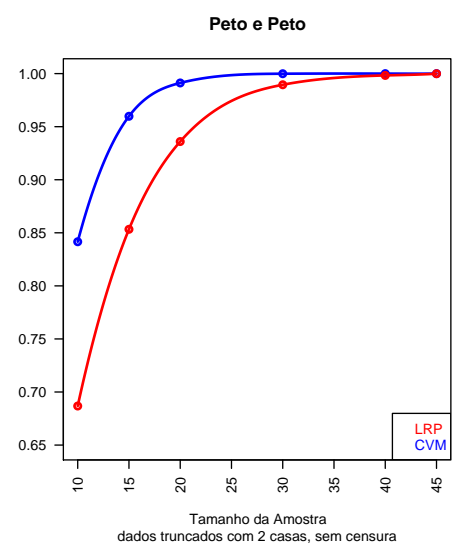

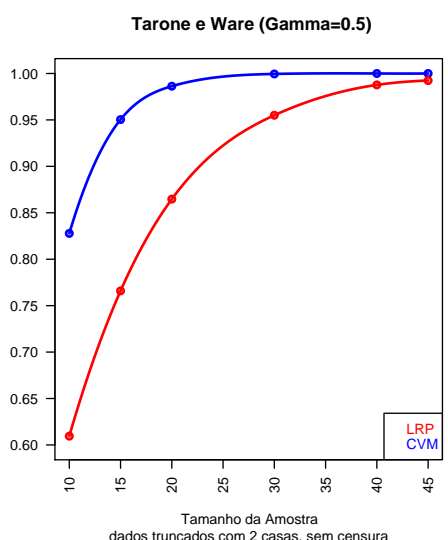

Tamanho da Amostra
dados truncados com 2 casas, sem censura

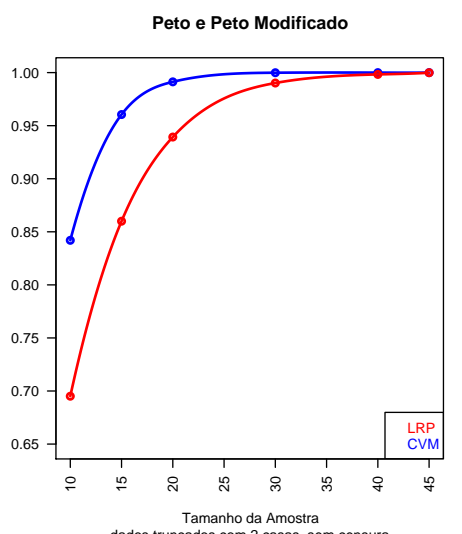

Tamanho da Amostra
dados truncados com 2 casas, sem censura

Figura 4.48: Gráfico para dados truncados com 2 casas na ausência de censura.

Nesta situação, estamos no caso em que truncamos os dados com duas casas depois da vírgula e ausência de censura. Note que, o teste de Cramér-von Mises têm poder maior que o de Log-rank Ponderado para qualquer função de ponderação. Observamos também que, o poder aumenta gradativamente, quando aumentamos o truncamento das casas decimais. 


\begin{tabular}{|c|c|c|c|c|c|c|c|c|c|c|c|}
\hline \multirow[b]{2}{*}{ TA } & \multirow[b]{2}{*}{ Peso } & \multirow[b]{2}{*}{ CVM } & \multirow[b]{2}{*}{ LRP } & \multicolumn{4}{|c|}{ Caso Truncado 2 casas na presença de censura } & \multirow[b]{2}{*}{ CVM } & \multirow[b]{2}{*}{ LRP } & \multirow[b]{2}{*}{$\%$ cens 1} & \multirow[b]{2}{*}{$\%$ cens 2} \\
\hline & & & & Peso & CVM & LRP & Peso & & & & \\
\hline 10 & \multirow{6}{*}{ Log-Rank } & 0,7761 & 0,4782 & \multirow{6}{*}{$\begin{array}{c}\text { Tarone e Ware } \\
\quad \gamma=0,5\end{array}$} & 0,7993 & 0,6674 & \multirow{6}{*}{ Peto e Peto } & 0,8472 & 0,6898 & \multirow{6}{*}{23,4} & \multirow{6}{*}{33,4} \\
\hline 15 & & 0,9052 & 0,5778 & & 0,9363 & 0,8259 & & 0,9623 & 0,8544 & & \\
\hline 20 & & 0,9622 & 0,6679 & & 0,9851 & 0,9202 & & 0,9911 & 0,9336 & & \\
\hline 30 & & 0,9962 & 0,7863 & & 0,9984 & 0,9832 & & 0,9998 & 0,9893 & & \\
\hline 40 & & 0,9995 & 0,8679 & & 0,9999 & 0,9969 & & 1 & 0,9979 & & \\
\hline 45 & & 1 & 0,8958 & & 1 & 0,9993 & & 1 & 0,9997 & & \\
\hline 10 & \multirow{6}{*}{ Gehan } & 0,8477 & 0,7047 & \multirow{6}{*}{$\begin{array}{c}\text { Fleming e Harrington } \\
\gamma=0,5 \text { e } \rho=2\end{array}$} & 0,8181 & 0,6577 & \multirow{6}{*}{ Peto e Peto Modificado } & 0,8482 & 0,6984 & \multirow{6}{*}{23,4} & \multirow{6}{*}{33,4} \\
\hline 15 & & 0,9628 & 0,8643 & & 0,9362 & 0,809 & & 0,9626 & 0,8615 & & \\
\hline 20 & & 0,9913 & 0,9391 & & 0,9803 & 0,9002 & & 0,9913 & 0,9383 & & \\
\hline 30 & & 0,9998 & 0,9904 & & 0,9988 & 0,9762 & & 0,9998 & 0,9904 & & \\
\hline 40 & & 1 & 0,998 & & 0,9998 & 0,9946 & & 1 & 0,9982 & & \\
\hline 45 & & 1 & 0,9997 & & 1 & 0,9978 & & 1 & 0,9997 & & \\
\hline
\end{tabular}

Tabela 4.40: Dados truncados com 2 casas na presença de censura.

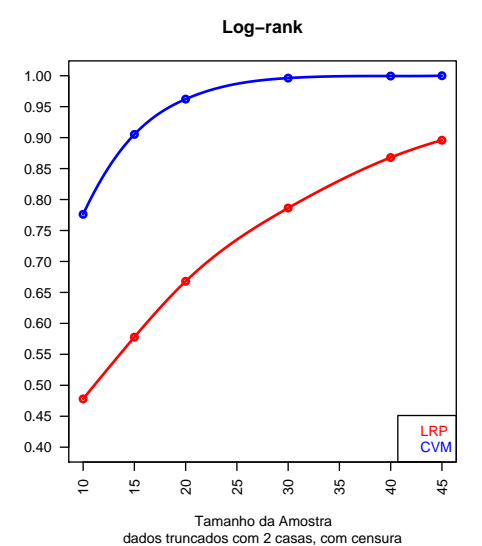

dados truncados com 2 casas, com censura

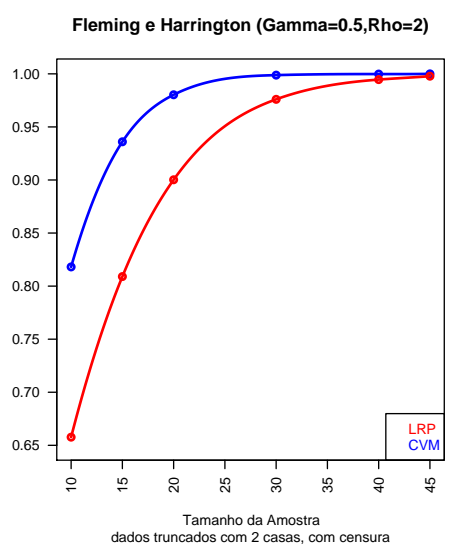

Gehan

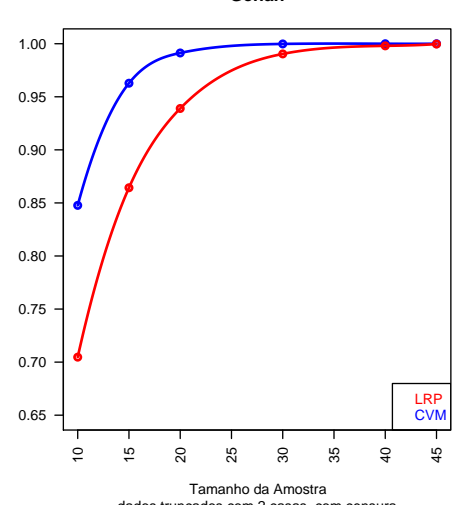

Tamanho da Amostra
dados truncados com 2 casas, com censura

Peto e Peto

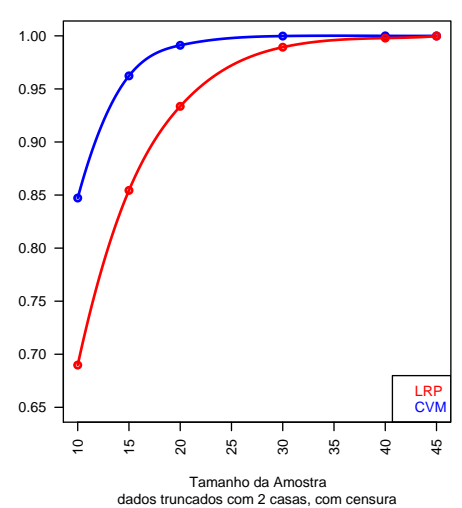

Tarone e Ware (Gamma=0.5)

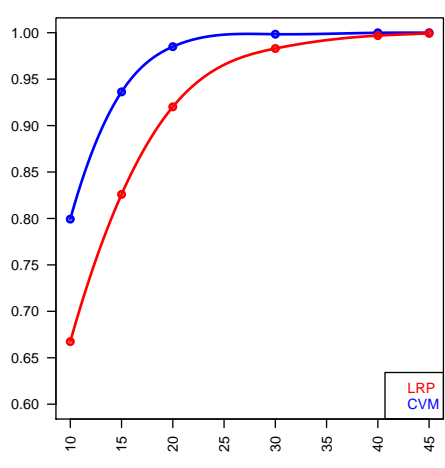

Tamanho da Amostra
dados truncados com 2 casas, com censura

Peto e Peto Modificado

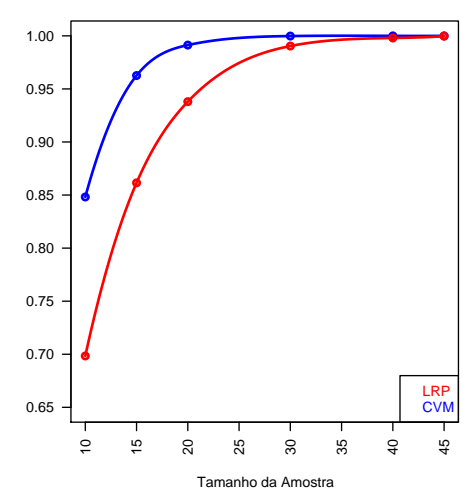

Tamanho da Amostra
dados truncados com 2 casas, com censura

Figura 4.49: Gráfico para dados truncados com 2 casas na presença de censura.

Na situação em que censuramos os dados, notamos um aumento imperceptível para as duas estatísticas, fato devido a porcentagem baixa de censura. Um aumento significativo do poder ocorre quando temos uma porcentagem alta de censura e este fato pode ser visto na seção (4.3), em que censuramos aproximadamente $50 \%$ dos dados. À medida que aumentamos as casas decimais, aumentamos bastante o número de categorias. 
Agora, no segundo experimento, tomamos $W^{n_{1}}$ com distribuição Weibull com parâmetros de forma $\delta=$ 2 e de escala $\alpha=100$, em seguida tomamos outra $W^{n_{2}}$ com distribuição Exponencial $\lambda=0,01$. Assim como no primeiro experimento, avaliamos $W^{n_{1}}$ e $W^{n_{2}}$ sob a hipótese nula $H_{0}$, em seguida, avaliamos o comportamento da função poder em relação aos tamanhos de amostra. O gráfico das funções de intensidade das duas amostras estão na figura (4.50).

\section{Taxas de Falha}

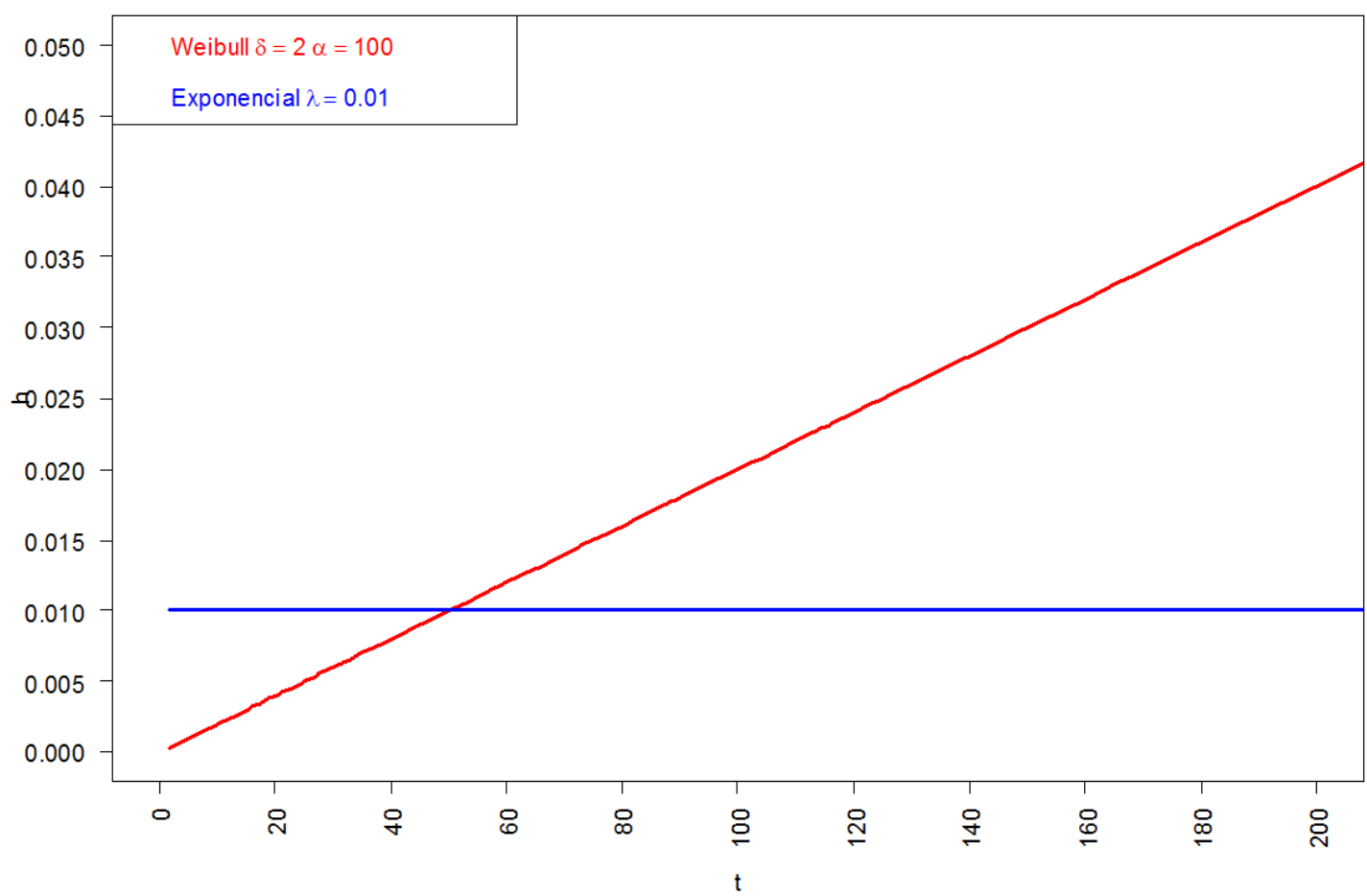

Figura 4.50: Taxa de falha das distribuições Weibull e Exponencial para o $2^{\circ}$ experimento.

Na primeira situação, estamos sob a hipótese nula e vamos seguir o seguinte algoritmo:

1. Fixamos um nível de significância $\alpha=0,05$;

2. Geramos $W^{(1)}, W^{(2)} \sim \operatorname{Wei}(\delta=2, \alpha=100)$ de tamanho TA dado que a hipótese nula é verdadeira;

3. Escolhemos uma das funções de ponderação descrita na tabela (3.1);

4. No caso com censura, temos censura à direita fixado em 100;

5. Realizamos o teste de hipótese usando valor crítico ao nível de significância $\alpha$;

6. Verificamos se cometeu o erro do tipo I, isto é, se a hipótese nula foi rejeitada;

$$
y_{m}= \begin{cases}1, & \text { se } H_{0} \text { não é rejeitada ao nível de significância } \alpha \\ 0, & \text { caso contrário }\end{cases}
$$

7. Repetimos as etapas 2 a $4 m=10000$ vezes; 
8. Estimamos a probabilidade de cometer o erro do tipo I da seguinte forma:

$$
\hat{\alpha}=\frac{1}{m} \sum_{k=1}^{m} y_{k}
$$

\begin{tabular}{|c|c|c|c|c|c|c|c|c|c|c|c|c|}
\hline \multirow{4}{*}{ TA } & \multirow{4}{*}{ Tipo } & \multirow{4}{*}{ Peso } & \multicolumn{4}{|c|}{ RESULTADOS SOB $H_{0}$} & & & & & \multirow{2}{*}{\multicolumn{2}{|c|}{$\%$ Censura }} \\
\hline & & & \multirow{2}{*}{\multicolumn{4}{|c|}{$\begin{array}{c}\text { Ausência de Censura } \\
\text { Estatística }\end{array}$}} & \multirow{2}{*}{\multicolumn{4}{|c|}{$\begin{array}{c}\text { Presença de Censura } \\
\text { Estatística }\end{array}$}} & & \\
\hline & & & & & & & & & & & \multirow[t]{2}{*}{ pop1 } & \multirow[t]{2}{*}{ pop2 } \\
\hline & & & LRP & CVM & Renyi & CCVM & LRP & CVM & Renyi & CCVM & & \\
\hline \multirow{6}{*}{50} & \multirow{6}{*}{ Truncado } & Log-Rank & 0,0539 & 0,0519 & 0,0425 & \multirow{6}{*}{0,0522} & 0,0519 & 0,0539 & 0,0425 & \multirow{6}{*}{0,0522} & \multirow{6}{*}{40,00} & \multirow{6}{*}{40,00} \\
\hline & & Gehan & 0,0530 & 0,0528 & 0,0446 & & 0,0528 & 0,0530 & 0,0446 & & & \\
\hline & & Tarone e Ware $\gamma=0,5$ & 0,0534 & 0,0535 & 0,0447 & & 0,0535 & 0,0534 & 0,0447 & & & \\
\hline & & Fleming e Harrington $\gamma=0,5$ e $\rho=2$ & 0,0556 & 0,0540 & 0,0446 & & 0,0540 & 0,0556 & 0,0446 & & & \\
\hline & & Peto e Peto & 0,0530 & 0,0526 & 0,0446 & & 0,0526 & 0,0530 & 0,0446 & & & \\
\hline & & Peto e Peto Modificado & 0,0532 & 0,0528 & 0,0444 & & 0,0528 & 0,0532 & 0,0444 & & & \\
\hline \multirow{6}{*}{100} & \multirow{6}{*}{ Truncado } & Log-Rank & 0,0477 & 0,0519 & 0,0440 & & 0,0483 & 0,0510 & 0,0453 & & & \\
\hline & & Gehan & 0,0511 & 0,0488 & 0,0433 & & 0,0494 & 0,0513 & 0,0437 & & & \\
\hline & & Tarone e Ware $\gamma=0,5$ & 0,0488 & 0,0493 & 0,0446 & 0,0497 & 0,0499 & 0,0504 & 0,0442 & 0,0516 & 40,00 & 40,00 \\
\hline & & Fleming e Harrington $\gamma=0,5$ e $\rho=2$ & 0,0483 & 0,0481 & 0,0425 & & 0,0507 & 0,0516 & 0,0455 & & & \\
\hline & & Peto e Peto & 0,0508 & 0,0489 & 0,0434 & & 0,0494 & 0,0513 & 0,0437 & & & \\
\hline & & Peto e Peto Modificado & 0,0511 & 0,0488 & 0,0434 & & 0,0494 & 0,0512 & 0,0437 & & & \\
\hline & & Log-Rank & 0,0522 & 0,0539 & 0,0445 & & 0,0483 & 0,0510 & 0,0453 & & & \\
\hline & & Gehan & 0,0508 & 0,0529 & 0,0460 & & 0,0494 & 0,0513 & 0,0437 & & & \\
\hline 50 & 1 casa & Tarone e Ware $\gamma=0,5$ & 0,0518 & 0,0524 & 0,0461 & 0,0519 & 0,0499 & 0,0504 & 0,0442 & 0,0516 & 40,00 & 40,00 \\
\hline & & Fleming e Harrington $\gamma=0,5$ e $\rho=2$ & 0,0534 & 0,0548 & 0,0459 & & 0,0507 & 0,0516 & 0,0455 & & & \\
\hline & & Peto e Peto & 0,0511 & 0,0527 & 0,0457 & & 0,0494 & 0,0513 & 0,0437 & & & \\
\hline & & Peto e Peto Modificado & 0,0510 & 0,0527 & 0,0460 & & 0,0494 & 0,0512 & 0,0437 & & & \\
\hline & & Log-Rank & 0,0493 & 0,0513 & 0,0475 & & 0,0519 & 0,0539 & 0,0425 & & & \\
\hline & & Gehan & 0,0494 & 0,0506 & 0,0458 & & 0,0528 & 0,0530 & 0,0446 & & & \\
\hline 100 & 1 casa & Tarone e Ware $\gamma=0,5$ & 0,0504 & 0,0503 & 0,0459 & 0,0512 & 0,0535 & 0,0534 & 0,0447 & 0,0522 & 40,00 & 40,00 \\
\hline & & Fleming e Harrington $\gamma=0,5$ e $\rho=2$ & 0,0515 & 0,0515 & 0,0484 & & 0,0540 & 0,0556 & 0,0446 & & & \\
\hline & & Peto e Peto & 0,0495 & 0,0509 & 0,0459 & & 0,0526 & 0,0530 & 0,0446 & & & \\
\hline & & Peto e Peto Modificado & 0,0494 & 0,0506 & 0,0458 & & 0,0528 & 0,0532 & 0,0444 & & & \\
\hline & & Log-Rank & 0,0531 & 0,0519 & 0,0425 & & 0,0522 & 0,0541 & 0,0448 & & & \\
\hline & & Gehan & 0,0530 & 0,0528 & 0,0446 & & 0,0507 & 0,0527 & 0,0459 & & & \\
\hline 50 & 2 casas & Tarone e Ware $\gamma=0,5$ & 0,0532 & 0,0535 & 0,0447 & 0,0522 & 0,0523 & 0,0523 & 0,0459 & 0,0518 & 40,00 & 40,00 \\
\hline & & Fleming e Harrington $\gamma=0,5$ e $\rho=2$ & 0,0556 & 0,0520 & 0,0446 & & 0,0532 & 0,0554 & 0,0464 & & & \\
\hline & & Peto e Peto & 0,0530 & 0,0526 & 0,0446 & & 0,0514 & 0,0529 & 0,0457 & & & \\
\hline & & Peto e Peto Modificado & 0,0532 & 0,0528 & 0,0444 & & 0,0512 & 0,0527 & 0,0457 & & & \\
\hline & & Log-Rank & 0,0487 & 0,0519 & 0,0440 & & 0,0492 & 0,0514 & 0,0477 & & & \\
\hline & & Gehan & 0,0514 & 0,0488 & 0,0433 & & 0,0496 & 0,0508 & 0,0456 & & & \\
\hline 100 & 2 casas & Tarone e Ware $\gamma=0,5$ & 0,0488 & 0,0495 & 0,0446 & 0,0497 & 0,0505 & 0,0504 & 0,0459 & 0,0509 & 40,00 & 40,00 \\
\hline & & Fleming e Harrington $\gamma=0,5$ e $\rho=2$ & 0,0487 & 0,0481 & 0,0425 & & 0,0512 & 0,0514 & 0,0486 & & & \\
\hline & & Peto e Peto & 0,0510 & 0,0489 & 0,0434 & & 0,0497 & 0,0507 & 0,0459 & & & \\
\hline & & Peto e Peto Modificado & 0,0515 & 0,0489 & 0,0434 & & 0,0495 & 0,0507 & 0,0457 & & & \\
\hline & & Log-Rank & 0,0539 & 0,0519 & 0,0425 & & 0,0522 & 0,0540 & 0,0448 & & & \\
\hline & & Gehan & 0,0530 & 0,0512 & 0,0449 & & 0,0507 & 0,0526 & 0,0459 & & & \\
\hline 50 & Contínuo & Tarone e Ware $\gamma=0,5$ & 0,0522 & 0,0535 & 0,0447 & 0,0522 & 0,0524 & 0,0522 & 0,0459 & 0,0518 & 40,00 & 40,00 \\
\hline & & Fleming e Harrington $\gamma=0,5$ e $\rho=2$ & 0,0562 & 0,0544 & 0,0448 & & 0,0534 & 0,0556 & 0,0466 & & & \\
\hline & & Peto e Peto & 0,0520 & 0,0526 & 0,0446 & & 0,0501 & 0,0529 & 0,0458 & & & \\
\hline & & Peto e Peto Modificado & 0,0512 & 0,0528 & 0,0444 & & 0,0518 & 0,0526 & 0,0458 & & & \\
\hline & & Log-Rank & 0,0497 & 0,0522 & 0,0440 & & 0,0495 & 0,0511 & 0,0477 & & & \\
\hline & & Gehan & 0,0511 & 0,0493 & 0,0433 & & 0,0495 & 0,0507 & 0,0457 & & & \\
\hline 100 & Contínuo & Tarone e Ware $\gamma=0,5$ & 0,0478 & 0,0499 & 0,0446 & 0,0498 & 0,0501 & 0,0507 & 0,0459 & 0,0519 & 40,00 & 40,00 \\
\hline & & Fleming e Harrington $\gamma=0,5$ e $\rho=2$ & 0,0495 & 0,0488 & 0,0425 & & 0,0515 & 0,0516 & 0,0498 & & & \\
\hline & & Peto e Peto & 0,0508 & 0,0498 & 0,0433 & & 0,0491 & 0,0507 & 0,0479 & & & \\
\hline & & Peto e Peto Modificado & 0,0512 & 0,0483 & 0,0484 & & 0,0498 & 0,0509 & 0,0467 & & & \\
\hline
\end{tabular}

Tabela 4.41: Resultados sob $H_{0}$ para o $2^{\circ}$ experimento.

No segundo experimento, quando estamos sob a hipótese nula, as estatísticas CVM, LRP, Renyi e CCVM estão muito próximas ao nível de significância nominal $\alpha=0,05$, quando truncamos os dados totalmente, com uma casa, com duas casas e no caso puramente contínuo. Notamos que para este experimento os testes de Renyi e CCVM também têm comportamento satisfatório tanto quanto as propostas neste trabalho, fato devido a maior variabilidade dos dados, pois neste experimento usamos os parâmetros de 
escala 2 e forma 100 para a distribuição Weibull, causando aumento do intervalo de dados. Assim, utilizamos as quatro estatísticas para avaliar o cruzamento das funções de intensidade nos casos de truncamento dos dados. Neste experimento, usamos os tamanhos de amostra TA de 50 e 100 na ausência e presença de censura. No caso de censura dos dados usamos censura à direita fixado em 100. A seguir, apresentamos o algoritmo.

1. Fixamos um nível de significância $\alpha=0,05$;

2. Escolhemos uma das funções de ponderação descrita na tabela (3.1);

3. Geramos populações de tamanho da amostra TA e definimos duas populações com distribuições:

- $W^{(1)} \sim \operatorname{Wei}(\delta=2 ; \alpha=100)$;

- $W^{(2)} \sim \operatorname{Exp}(\lambda=0,01)$ dado que a hipótese nula é falsa;

4. Para o caso com censura, temos censura à direita fixado em 100;

5. Realizamos o teste de hipótese usando valor crítico ao nível de significância $\alpha$;

6. Para estimarmos a função poder tomamos:

$$
y_{m}=\left\{\begin{array}{cc}
1, & \text { se } H_{0} \text { foi rejeitada ao nível de significância } \alpha \\
0, & \text { caso contrário }
\end{array}\right.
$$

7. Repetimos as etapas 2 a $4 m=10000$ vezes;

8. Estimamos o poder do teste da seguinte forma:

$$
\text { Poder }=\frac{1}{m} \sum_{k=1}^{m} y_{k}
$$

Os resultados do último experimento do capítulo é apresentada na tabela (4.42). No caso de ausência de censura observamos que CCVM tem poder levemente maior nas quatro situações (truncamento total, 1 casa, 2 casas, puramente contínuo) em comparação com o teste de Renyi e CVM usando os pesos Gehan, Peto e Peto e Peto e Peto Modificado. Já LRP não se aproxima de 1 para tamanhos da amostra até 100, fato devido ao cruzamento severo das funções de intensidade, pois neste experimento temos cruzamento em $t=2$ para o caso de truncamento total e em torno de $t=2,014$ para os demais casos. No caso de truncamento total temos $63,2 \%(60 \%)$ de ser 2 e 36,8\% (40\%) de estar localizado após o ponto do cruzamento $t=2$. Já nos demais casos temos 86,5\% (85\%) de ser 2,014 e 13,5\% (15\%) de estar localizado após o ponto do cruzamento $t=2,014$.

Agora, no caso de censura dos dados temos que CVM e Renyi têm maior poder e comportamentos similares que os demais. Notamos também, com censura dos dados, CCVM e LRP não se aproxima de 1 
para tamanhos de amostra até 100, porém, temos que LRP dobra seu valor em comparação com o caso de ausência de censura, ao contrário de CCVM que diminui substancialmente seu valor. A censura do tipo I fixada em 100 faz com que o ponto do cruzamento seja antecipado para $t=1$ no caso de truncamento total e $t=1,31$ nos demais casos, diminuindo o cancelamento das diferenças positivas e negativas entre as duas funções intensidade estimadas, fato este a causa da melhora do poder para LRP.

\begin{tabular}{|c|c|c|c|c|c|c|c|c|c|c|c|c|}
\hline \multirow{4}{*}{ TA } & \multicolumn{10}{|c|}{ Resultados para cruzamento das funções de intensidade na ausência e presença de censura } & \multirow{2}{*}{\multicolumn{2}{|c|}{$\%$ Censura }} \\
\hline & \multirow{3}{*}{ Tipo } & \multirow{3}{*}{ Peso } & \multirow{2}{*}{\multicolumn{4}{|c|}{$\begin{array}{c}\text { Ausência de Censura } \\
\text { Estatística }\end{array}$}} & \multirow{2}{*}{\multicolumn{4}{|c|}{$\begin{array}{c}\text { Presença de Censura } \\
\text { Estatística }\end{array}$}} & & \\
\hline & & & & & & & & & & & \multirow[t]{2}{*}{ pop1 } & \multirow[t]{2}{*}{ pop2 } \\
\hline & & & CVM & LRP & Renyi & CCVM & CVM & LRP & Renyi & CCVM & & \\
\hline \multirow{6}{*}{50} & \multirow{6}{*}{ Truncado } & Log-Rank & 0,1975 & 0,0934 & 0,4633 & \multirow{6}{*}{0,4876} & 0,4373 & 0,1232 & 0,4884 & \multirow{6}{*}{0,1457} & \multirow{6}{*}{38,0} & \multirow{6}{*}{30,0} \\
\hline & & Gehan & 0,6162 & 0,1283 & 0,6083 & & 0,6435 & 0,2770 & 0,6122 & & & \\
\hline & & Tarone e Ware $\gamma=0,5$ & 0,4606 & 0,0530 & 0,5492 & & 0,5596 & 0,1930 & 0,5588 & & & \\
\hline & & Fleming e Harrington $\gamma=0,5$ e $\rho=2$ & 0,3959 & 0,0859 & 0,3863 & & 0,4146 & 0,1422 & 0,3927 & & & \\
\hline & & Peto e Peto & 0,6112 & 0,1234 & 0,6071 & & 0,6417 & 0,2733 & 0,6105 & & & \\
\hline & & Peto e Peto Modificado & 0,6149 & 0,1275 & 0,6074 & & 0,6429 & 0,2755 & 0,6113 & & & \\
\hline \multirow{6}{*}{100} & \multirow{6}{*}{ Truncado } & Log-Rank & 0,4256 & 0,1951 & 0,8116 & & 0,7307 & 0,1676 & 0,8235 & & & \\
\hline & & Gehan & 0,8991 & 0,2080 & 0,9089 & & 0,9145 & 0,4666 & 0,9061 & & & \\
\hline & & Tarone e Ware $\gamma=0,5$ & 0,7773 & 0,0511 & 0,8759 & 0,9226 & 0,8578 & 0,3077 & 0,8775 & 0,2592 & 38,0 & 30,0 \\
\hline & & Fleming e Harrington $\gamma=0,5$ e $\rho=2$ & 0,6598 & 0,1098 & 0,6769 & & 0,6764 & 0,2032 & 0,6747 & & & \\
\hline & & Peto e Peto & 0,8979 & 0,2025 & 0,9085 & & 0,9136 & 0,4636 & 0,9060 & & & \\
\hline & & Peto e Peto Modificado & 0,8990 & 0,2071 & 0,9086 & & 0,9142 & 0,4664 & 0,9061 & & & \\
\hline & & Log-Rank & 0,1981 & 0,0936 & 0,4636 & & 0,4421 & 0,1251 & 0,4994 & & & \\
\hline & & Gehan & 0,6112 & 0,1287 & 0,6084 & & 0,6474 & 0,2776 & 0,6201 & & & \\
\hline 50 & 1 casa & Tarone e Ware $\gamma=0,5$ & 0,4608 & 0,0533 & 0,5496 & 0,4882 & 0,5649 & 0,1956 & 0,5685 & 0,1535 & 37,0 & 36,0 \\
\hline & & Fleming e Harrington $\gamma=0,5$ e $\rho=2$ & 0,3962 & 0,0862 & 0,3867 & & 0,4196 & 0,1452 & 0,4027 & & & \\
\hline & & Peto e Peto & 0,6118 & 0,1234 & 0,6078 & & 0,6444 & 0,2744 & 0,6189 & & & \\
\hline & & Peto e Peto Modificado & 0,6151 & 0,1277 & 0,6085 & & 0,6465 & 0,2773 & 0,6193 & & & \\
\hline & & Log-Rank & 0,4264 & 0,1956 & 0,8118 & & 0,7369 & 0,1716 & 0,8328 & & & \\
\hline & & Gehan & 0,8997 & 0,2088 & 0,9140 & & 0,9154 & 0,4679 & 0,9109 & & & \\
\hline 100 & 1 casa & Tarone e Ware $\gamma=0,5$ & 0,7781 & 0,0516 & 0,8763 & 0,9231 & 0,8606 & 0,3096 & 0,8833 & 0,2749 & 37,0 & 36,0 \\
\hline & & Fleming e Harrington $\gamma=0,5$ e $\rho=2$ & 0,6602 & 0,1104 & 0,6812 & & 0,688 & 0,2086 & 0,6944 & & & \\
\hline & & Peto e Peto & 0,8984 & 0,2029 & 0,9089 & & 0,9149 & 0,4659 & 0,9105 & & & \\
\hline & & Peto e Peto Modificado & 0,8993 & 0,2081 & 0,9109 & & 0,9154 & 0,4677 & 0,9108 & & & \\
\hline & & Log-Rank & 0,1999 & 0,0902 & 0,4708 & & 0,4433 & 0,1252 & 0,5013 & & & \\
\hline & & Gehan & 0,6094 & 0,1325 & 0,6117 & & 0,6487 & 0,2787 & 0,6223 & & & \\
\hline 50 & 2 casas & Tarone e Ware $\gamma=0,5$ & 0,4563 & 0,0533 & 0,5525 & 0,4825 & 0,5659 & 0,1956 & 0,5708 & 0,1545 & 37,0 & 36,0 \\
\hline & & Fleming e Harrington $\gamma=0,5$ e $\rho=2$ & 0,3972 & 0,0917 & 0,3948 & & 0,4212 & 0,1457 & 0,4052 & & & \\
\hline & & Peto e Peto & 0,6051 & 0,1275 & 0,6098 & & 0,6459 & 0,2751 & 0,6212 & & & \\
\hline & & Peto e Peto Modificado & 0,6086 & 0,1324 & 0,6113 & & 0,6481 & 0,2781 & 0,6215 & & & \\
\hline & & Log-Rank & 0,4261 & 0,1957 & 0,8124 & & 0,7375 & 0,1706 & 0,8327 & & & \\
\hline & & Gehan & 0,8993 & 0,2082 & 0,9112 & & 0,9154 & 0,4672 & 0,9112 & & & \\
\hline 100 & 2 casas & Tarone e Ware $\gamma=0,5$ & 0,7769 & 0,0518 & 0,8764 & 0,9251 & 0,8602 & 0,3091 & 0,8836 & 0,2759 & 37,0 & 36,0 \\
\hline & & Fleming e Harrington $\gamma=0,5$ e $\rho=2$ & 0,6601 & 0,1103 & 0,6782 & & 0,6881 & 0,2093 & 0,6963 & & & \\
\hline & & Peto e Peto & 0,8981 & 0,2121 & 0,9076 & & 0,9151 & 0,4649 & 0,9111 & & & \\
\hline & & Peto e Peto Modificado & 0,8993 & 0,2084 & 0,9089 & & 0,9153 & 0,4667 & 0,9112 & & & \\
\hline & & Log-Rank & 0,1963 & 0,0912 & 0,4699 & & 0,4422 & 0,1253 & 0,5007 & & & \\
\hline & & Gehan & 0,6131 & 0,1318 & 0,6144 & & 0,6476 & 0,2775 & 0,6206 & & & \\
\hline 50 & Contínuo & Tarone e Ware $\gamma=0,5$ & 0,4571 & 0,0582 & 0,5583 & 0,4918 & 0,5648 & 0,1954 & 0,5698 & 0,1544 & 37,0 & 36,0 \\
\hline & & Fleming e Harrington $\gamma=0,5 \mathrm{e} \rho=2$ & 0,3991 & 0,0916 & 0,4003 & & 0,4202 & 0,1454 & 0,4043 & & & \\
\hline & & Peto e Peto & 0,6088 & 0,1275 & 0,6138 & & 0,6447 & 0,2739 & 0,6197 & & & \\
\hline & & Peto e Peto Modificado & 0,6124 & 0,1314 & 0,6142 & & 0,6469 & 0,2768 & 0,6202 & & & \\
\hline & & Log-Rank & 0,4265 & 0,1959 & 0,8126 & & 0,7376 & 0,1707 & 0,8329 & & & \\
\hline & & Gehan & 0,8998 & 0,2084 & 0,9116 & & 0,9154 & 0,4666 & 0,9114 & & & \\
\hline 100 & Contínuo & Tarone e Ware $\gamma=0,5$ & 0,7776 & 0,0526 & 0,8751 & 0,9226 & 0,8602 & 0,3086 & 0,8838 & 0,2756 & 37,0 & 36,0 \\
\hline & & Fleming e Harrington $\gamma=0,5 \mathrm{e} \rho=2$ & 0,6613 & 0,1165 & 0,6771 & & 0,6879 & 0,2091 & 0,6967 & & & \\
\hline & & Peto e Peto & 0,8983 & 0,2126 & 0,9086 & & 0,9151 & 0,4644 & 0,9113 & & & \\
\hline & & Peto e Peto Modificado & 0,8994 & 0,2078 & 0,9089 & & 0,9154 & 0,4662 & 0,9114 & & & \\
\hline
\end{tabular}

Tabela 4.42: Resultados para cruzamento das funções de intensidade na ausência e presença de censura do $2^{\circ}$ experimento.

O intuito desta seção foi medir o desempenho dos testes para um número infinito enumerável de categorias. Observando os resultados dos estudos de simulação, as duas estatísticas têm um bom desempenho para $\mathcal{K} \subseteq \mathbb{N}$ com $\mathcal{K}$ infinito enumerável de categorias.

Resumindo os estudos de simulação desta seção, observamos no primeiro experimento que tanto a es- 
tatística de CVM, quanto LRP, têm bom desempenho para um número infinito enumerável de categorias e o poder cresce à medida que aumentamos as casas decimais. Porém, uma observação importante é que o poder para CVM cresce em proporção maior que o de LRP à medida que aumentamos as casas decimais. Neste mesmo experimento vimos que os testes de CCVM e Renyi não se aproximam da probabilidade nominal de $(\alpha=0,05)$, fato devido a pequena variabilidade neste experimento, ocorrendo uma concentração dos dados, ou seja, diminuindo bastante o intervalo dos dados e como isso retiramos elas da avaliação do poder.

No segundo experimento, todas as estatísticas se aproximam do nível de significância nominal sob a hipótese nula, fato devido a maior variabilidade neste experimento, com isso aumentando o intervalo dos dados. Na avaliação do poder destes testes, notamos comportamento satisfatório de CVM, CCVM e Renyi para o caso de cruzamento severo das funções de intensidade, não sendo satisfatório apenas para LRP. Um balanço geral desta seção é que para CVM tem comportamento satisfatório para cruzamento das funções de intensidade independente dos parâmetros escolhidos para as distribuições de probabilidade, não ocorrendo o mesmo com as estatísticas propostas no trabalho de Klein e Moeschberger (1997)[15] que no primeiro experimento não tem bom comportamento sob a hipótese nula. 


\section{Capítulo 5}

\section{Aplicações}

Afim de sedimentar os conceitos e resultados obtidos nas seções anteriores, vamos aplicar as estatísticas propostas em alguns exemplos, que serviram de motivação para este trabalho. A primeira aplicação é a principal motivação deste trabalho, que é baseado no cruzamento das funções de intensidade. A principal dificuldade nestes dados são que as diferenças iniciais em favor de uma população (antes do cruzamento) são canceladas após o cruzamento pelas diferenças em favor da outra população, isto faz com que os testes tradicionais tenham dificuldade em detectar diferenças.

A seguir, aplicamos os testes propostos para análise da evasão dos alunos de graduação da USP, que também apresenta cruzamento das funções de intensidade. Estes dados foram extraídos do trabalho de Pelissari [18].

\subsection{Aplicação para grupo de tumores gastrointestinais}

Os dados do livro de Klein e Moeschberger (1997)[15] são referentes a um Grupo de Estudo de Tumores Gastrointestinais (1982) que reportou os resultados do estudo comparando a quimioterapia versus quimioterapia combinada com a radioterapia no tratamento do câncer gástrico. Como dito na seção (1.1), a principal motivação deste conjunto de dados é o cruzamento das funções de intensidade, em que temos dificuldade em detectar diferenças entre as populações. Na seção (4.2.2), comprovamos através do estudo de simulação, que a função poder empírica do teste para a estatística de Cramér-von Mises é maior que o teste de Log-Rank Ponderado. Assim, vamos tomar esta aplicação para comprovar os resultados obtidos do estudo de simulação feito no capítulo anterior, comparando os testes propostos com os testes enunciados em [15] como por exemplo, o teste de Renyi. Neste exemplo, não utilizamos o teste de Log-rank Modificado pelo número grande de categorias. O tempo de sobrevivência é medido em dias, para os 45 pacientes em cada tratamento. Os dados da aplicação estão resumidos na tabela (5.1). 


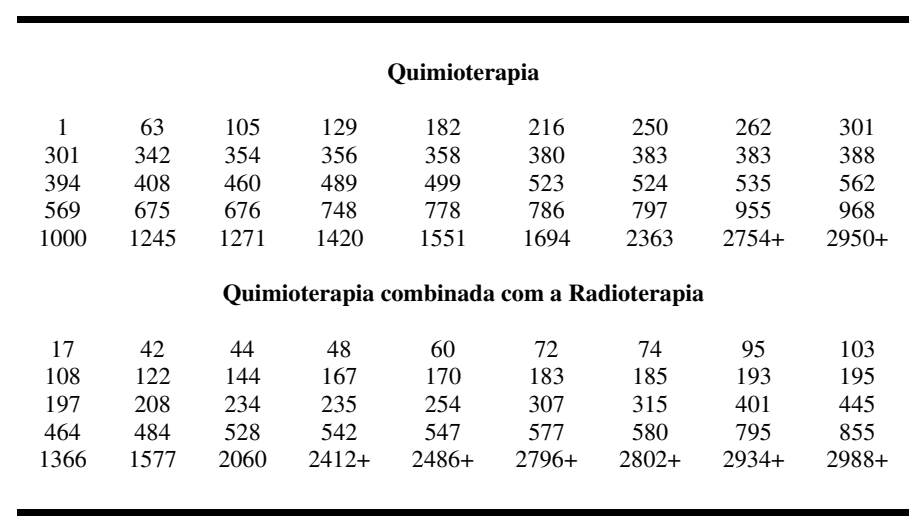

Tabela 5.1: Aplicação do livro de Klein e Moeschberger (1997)[15] páginas 18 e 19.
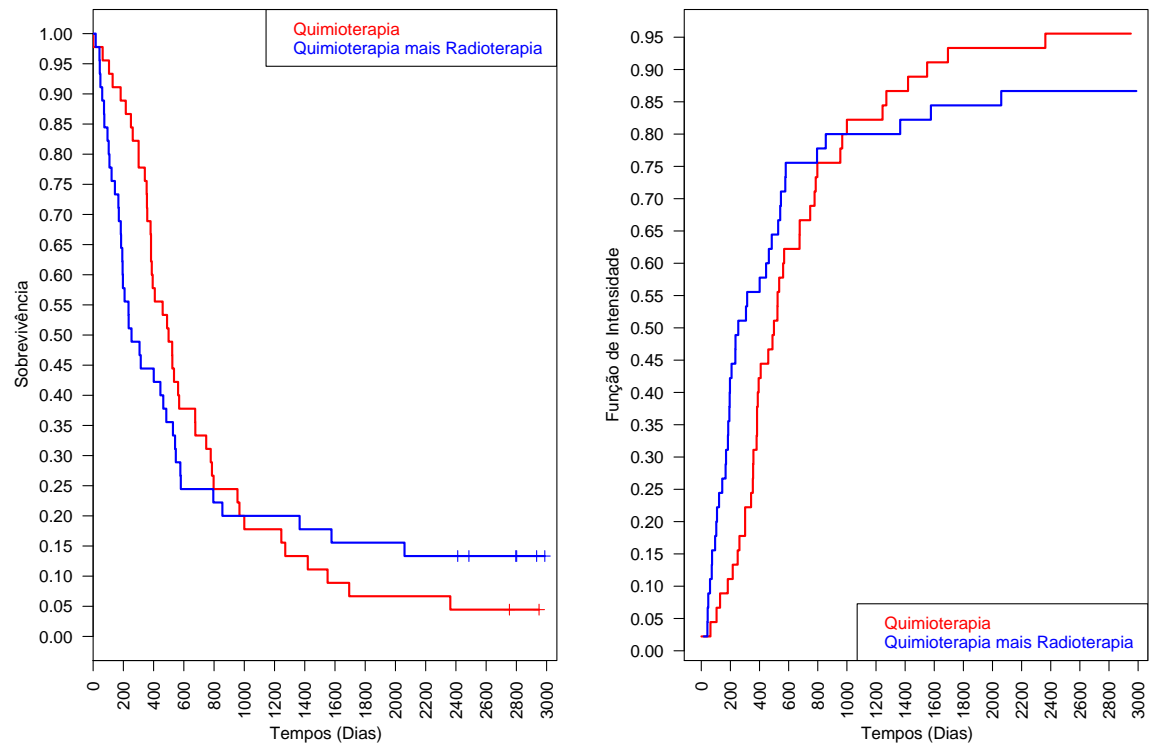

Figura 5.1: Estimativa para a função de sobrevivência e função de intensidade.

Pelos gráficos da função de sobrevivência e das funções de intensidade, notamos um cruzamento severo (aproximadamente em 1000 dias) e pode causar uma dificuldade em detectar diferenças significativas entre $W_{1}$ e $W_{2}$. Porém, vamos comprovar isto com a aplicação dos testes. Na primeira comparação usamos os testes propostos em comparação com os testes apresentados em Klein e Moeschberger[15].

\begin{tabular}{|c|c|c|c|c|c|c|}
\hline Peso & CVM & $\begin{array}{c}\text { p-valor } \\
\text { LRP }\end{array}$ & Renyi & CVM & $\begin{array}{c}\text { Estatísticas } \\
\text { LRP }\end{array}$ & Renyi \\
\hline Log-Rank & 0,02855894 & 0,6227514 & 0,053 & 0,09259588 & 0,2420193 & 2,200066 \\
\hline Gehan & 0,004171 & 0,0472824 & 0,007 & 0,02476226 & 3,9353101 & 2,951879 \\
\hline Tarone e Ware $\gamma=0,5$ & 0,00647284 & 0,1632943 & 0,01 & 0,04621725 & 1,9434511 & 2,677299 \\
\hline Fleming e Harrington $\gamma=0,5$ e $\rho=2$ & 0,00264633 & 0,0520414 & 0,008 & 0,00026786 & 3,7744384 & 2,908441 \\
\hline Peto e Peto & 0,00414091 & 0,0495645 & 0,007 & 0,025245 & 3,8561295 & 2,946575 \\
\hline Peto e Peto Modificado & 0,00410887 & 0,0471301 & 0,007 & 0,0237622 & 3,9407391 & 2,954641 \\
\hline
\end{tabular}

Tabela 5.2: Resumo com as Estatísticas e p-valores para diversos testes estatísticos.

Agora, mesmo que com perda na precisão das estimativas quando utilizamos um modelo contínuo em 
dados discretos, comparamos os resultados utilizando o modelo Weibull.

\begin{tabular}{ccccc}
\hline Modelo Weibull & Estimativa & Desvio-padrão & $\mathbf{z}$ & p-valor \\
(Intercept) & 6,58 & 0,179 & 36,59 & 0 \\
Tratamento:QuimioRadio & 0,035 & 0,26 & 0,135 & 0,892 \\
Log(scale) & 0,158 & 0,087 & 1,82 & 0,068 \\
\hline
\end{tabular}

Tabela 5.3: Estimativas dos parâmetros para o modelo Weibull.

Como dito anteriormente, o cruzamento severo das funções de intensidade causam uma dificuldade em detectar diferenças entre os tratamentos. O teste de Renyi apresentado em Klein e Moeschberger[15] não detecta diferença significativa ao nível de significância $\alpha=0,05$, para o peso log-rank $u\left(n^{\star}, \ell\right)=1$, e sim para as demais funções de ponderação, porém estes resultados podem estar distorcidos, pois nos resultados de simulações este teste não não se aproxima do nível de significância nominal quando estamos sob a hipótese nula $H_{0}$. Já para LRP não detecta diferenças para o peso log-rank, Tarone e Ware e Fleming e Harrington. Uma observação importante é que para CVM detectamos diferença significativa para todos os pesos (ver tabela 5.2). Utilizando o modelo Weibull usados em dados contínuos também não detectamos diferença significativa (ver tabela 5.3). Assim, comprovamos que CVM pode detectar diferença significativa, mesmo em cruzamentos severos das funções de intensidade, fato visto graficamente. Intuitivamente, o fato do cruzamento é devido o desempenho a longo prazo ser melhor quando aplicamos a quimioterapia combinada com a radioterapia, enquanto a quimioterapia é melhor a curto prazo.

\subsection{Aplicação para Evasão de alunos de graduação da USP}

Nesta seção aplicamos as estatísticas proposta neste trabalho para o exemplo extraído do trabalho de Pelissari [18] referente aos dados dos alunos ingressantes por vestibular à partir de 2000 até 2003 no nível de graduação da Universidade de São Paulo - USP (Fonte: Júpter Web). Os alunos foram divididos em 5 categorias, conforme a última ocorrência no sistema Júpter Web.

\begin{tabular}{ccc}
\hline Status do aluno & Número de Alunos & Porcentagem \\
& & \\
\hline Em Andamento & 6602 & $21,18 \%$ \\
Conclusão & 18305 & $58,71 \%$ \\
Evasão & 4913 & $15,76 \%$ \\
Falecimento & 39 & $0,12 \%$ \\
Migração & 1317 & $4,23 \%$ \\
Total & 31176 & $100 \%$ \\
& & \\
\hline
\end{tabular}

Tabela 5.4: Status dos alunos.

O termo evasão foi definido pela Pró-Reitoria de graduação da USP como aluno que se desligou da USP a seu pedido ou por motivos administrativos ( 3 semestres sem matrícula ou jubilamento ${ }^{1}$ ). Enquanto o termo

\footnotetext{
${ }^{1}$ Este é um termo usado quando um aluno é desligado por não ter atingido desempenho satisfatório na universidade de acordo com
} 
migração é referido ao aluno que mudou de curso dentro da USP. De acordo com a tabela (5.4), concluímos que $21,18 \%$ dos alunos estão com o curso em andamento, 58,71\% concluíram e 15,76\% evadiram.

\begin{tabular}{|c|c|c|c|c|c|c|c|c|c|c|c|c|}
\hline Ano & Semestre & Evadiu & Não Evadiu & Sob risco & Semestre & Evadiu & Não Evadiu & Sob risco & Semestre & Evadiu & Não Evadiu & Sob risco \\
\hline 2000 & 1 & 285 & 874 & 1159 & 2 & 257 & 617 & 874 & 3 & 117 & 500 & 617 \\
\hline 2001 & & 179 & 798 & 977 & & 206 & 592 & 798 & & 105 & 487 & 592 \\
\hline 2002 & & 179 & 903 & 1082 & & 297 & 606 & 903 & & 125 & 481 & 606 \\
\hline 2003 & & 212 & 830 & 1042 & & 228 & 602 & 830 & & 117 & 485 & 602 \\
\hline Total & & 855 & 3405 & 4260 & & 988 & 2417 & 3405 & & 464 & 1953 & 2417 \\
\hline 2000 & 4 & 110 & 390 & 500 & 5 & 118 & 272 & 390 & 6 & 94 & 178 & 272 \\
\hline 2001 & & 101 & 386 & 487 & & 108 & 278 & 386 & & 86 & 192 & 278 \\
\hline 2002 & & 92 & 389 & 481 & & 103 & 286 & 389 & & 67 & 219 & 286 \\
\hline 2003 & & 104 & 381 & 485 & & 100 & 281 & 381 & & 60 & 221 & 281 \\
\hline Total & & 407 & 1546 & 1953 & & 429 & 1117 & 1546 & & 307 & 810 & 1117 \\
\hline 2000 & 7 & 68 & 110 & 178 & 8 & 47 & 63 & 110 & 9 & 63 & 0 & 63 \\
\hline 2001 & & 71 & 121 & 192 & & 59 & 62 & 121 & & 62 & 0 & 62 \\
\hline 2002 & & 81 & 138 & 219 & & 71 & 67 & 138 & & 67 & 0 & 67 \\
\hline 2003 & & 109 & 112 & 221 & & 73 & 39 & 112 & & 39 & 0 & 39 \\
\hline Total & & 329 & 481 & 810 & & 250 & 231 & 481 & & 231 & 0 & 231 \\
\hline
\end{tabular}

Tabela 5.5: Evasão dos alunos de graduação por semestre.

Na análise comparativa da evasão ao longo dos anos, consideramos apenas os alunos que evadiram e tomamos como base o ano de 2003, pois um aluno que ingressou em 2003 teve menos semestres para evadir em relação a um aluno que ingressou em 2000, 2001 ou 2002. Um aluno que ingressou em 2003 teve 10 semestres para evadir. Desta forma, comparamos a evasão entre os anos, considerando apenas os alunos que evadiram até o $9^{\circ}$ semestre. Além disso, a evasão é dividida por semestre, conforme os dados apresentados na tabela (5.5).

No primeiro momento, comparamos a evasão dos alunos para os anos de 2000 a 2003 . Note que, pela tabela (5.6) detectamos diferença significativa entre os anos de 2000 a 2003, tanto para os testes de Logrank ponderado, Modificado e o de Cramér-von Mises ao nível de significância de $\alpha=0,05$. Análises mais detalhadas, como período (Manhã, tarde, noite e integral), sexo (Masculino e Feminino) são estudas no trabalho de Pelissari [18]. Em nossa aplicação, vamos comparar os anos, como resumidos na tabela (5.5) e por área (Exatas, Humanas e Biológicas). A seguir, tomamos como ano base o ano de 2000 e comparamos ela com as demais.

\begin{tabular}{ccc}
\hline & Estatística & p-valor \\
\hline LRP & 0,02983645 & 0,0000057 \\
CVM & 17,56526 & 0,000540646 \\
MLR & 87,3182 & $3,9 \times 10^{-9}$ \\
& & \\
\hline
\end{tabular}

Tabela 5.6: Comparação entre os anos de 2000 a 2003.

Testando a homogeneidade de um número de populações com uma simples escolha entre aceitação e rejeição deixamos muitas perguntas sem resposta. Por exemplo, se a hipótese $H_{0}$ é rejeitada gostaríamos de obter mais informações sobre as diferenças entre as populações. Procedimentos de comparações múltiplas critérios da mesma. 

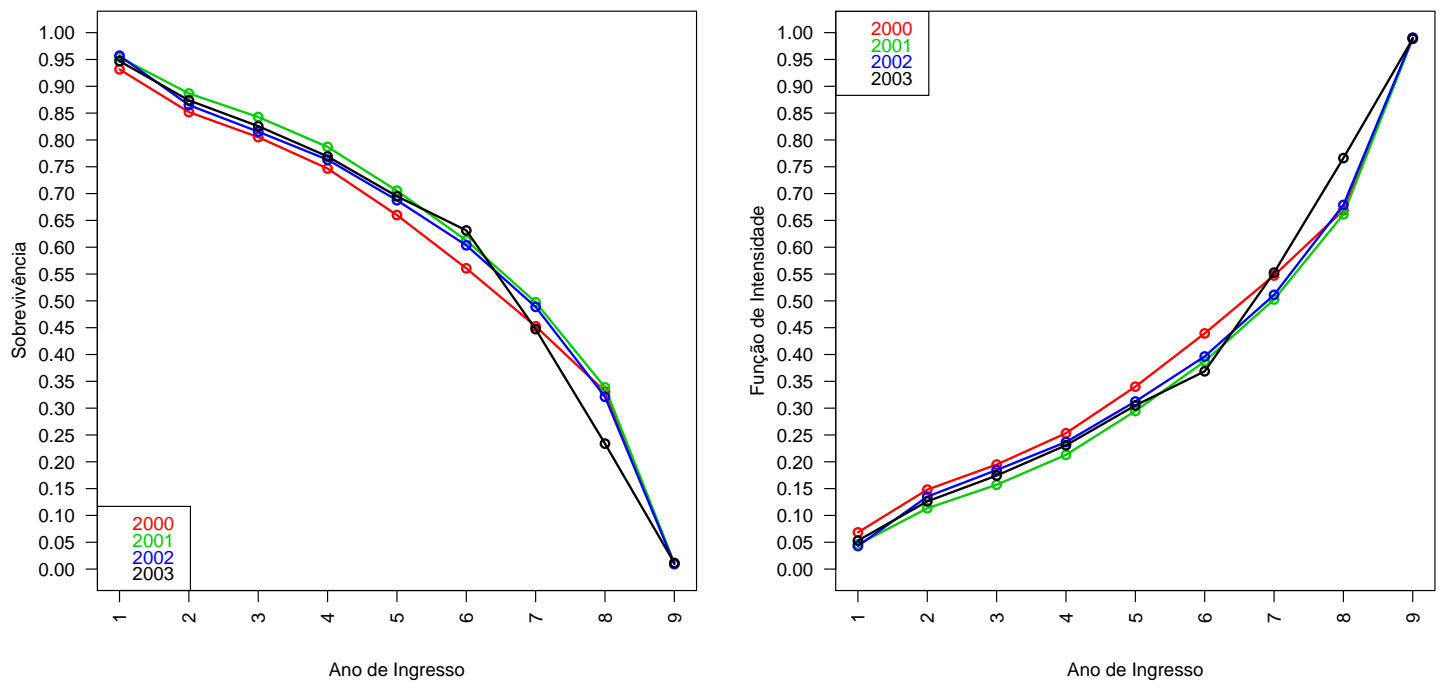

Figura 5.2: Gráfico da funções de sobrevivência e e das funções de intensidade.

(MCP) são técnicas úteis para detectar diferenças entre as populações em problemas de $\mathcal{J}$-amostras. Tais procedimentos têm sido desenvolvidos e eles tem sido objeto de consideráveis investigações (ver, Holm (1979)[13], Hochberg (1988) [11], e Benjamini Hochberg (1995) [12], Sarkar (1998) [20], Gao et al. (2008) [6] e Guo (2009)[6]), para isto, no controle da FWER usamos a estratégia de Hochberg [12] que é uma extensão do teste de Simes para avaliar os p-valores.

\begin{tabular}{|c|c|c|c|c|c|c|c|}
\hline \multirow[b]{2}{*}{ Comparação } & \multirow[b]{2}{*}{ FWER $\alpha_{i}$} & \multicolumn{2}{|c|}{ CVM } & \multicolumn{2}{|c|}{ MLR } & \multicolumn{2}{|c|}{ LRP } \\
\hline & & p-valor & Diferença & p-valor & Diferença & p-valor & Diferença \\
\hline $2000-2001$ & 0,016666667 & 0 & Sim & 0,00055 & Sim & 0,00004 & Sim \\
\hline $2000-2002$ & 0,025 & 0,02214 & Sim & 0 & Sim & 0,88281 & Não \\
\hline $2000-2003$ & 0,05 & 0,03844 & Sim & 0 & Sim & 0,30899 & Não \\
\hline
\end{tabular}

Tabela 5.7: Comparação 2 a 2 entre os anos de 2000 a 2003.

Portanto, pela tabela (5.7) concluímos que temos diferença significativa entre todas as combinações usando a estatística CVM e MLR. Agora, para a estatística de LRP não detectamos diferença significativa na comparação entre os anos de 2000 com 2002 e 2000 com 2003, todos ao nível de controle da FWER $\left(\alpha_{i}, \quad i=1,2\right.$ e 3$)$ descrito na tabela (5.7). Este último não é detectado por conta de um cruzamento da função de intensidade nestes dois anos (ver figura 5.3). Assim, com base nas conclusões obtidas pelas estatísticas CVM e MLR, temos diferenças significativas em suas funções de intensidade, fato visto graficamente (figura (5.2)), pois as taxas de evasão vão aumentando ao longo dos semestres com passar dos anos, exceto no ano de 2003, que oscilou seu comportamento à partir do $6^{\circ}$ semestre.

Agora, avaliamos o nível de evasão dos alunos em relação a área (Exatas, Humanas e Biológicas). Os dados estão resumidos na tabela (5.8). Assim como no estudo anterior, testamos a homogeneidade geral 


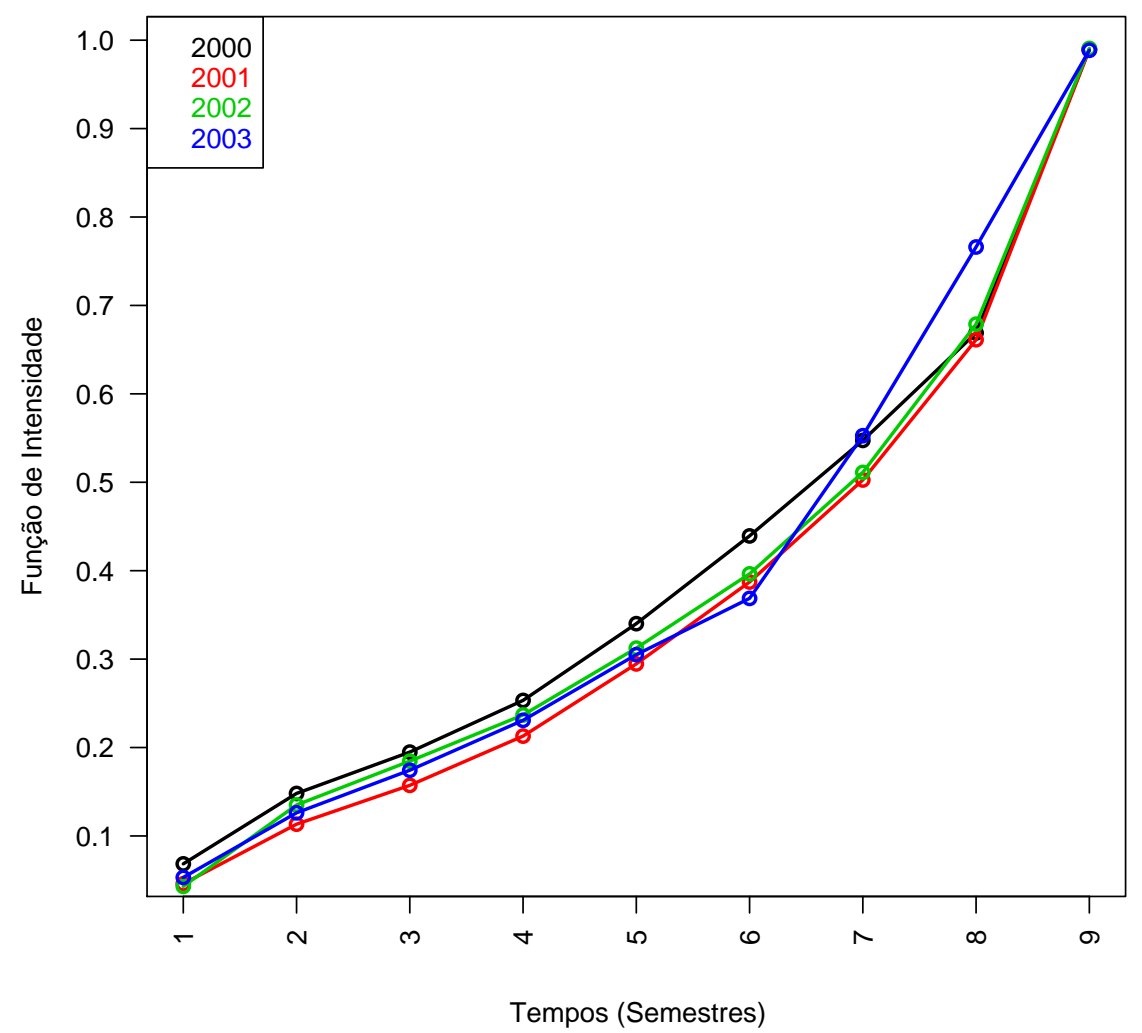

Figura 5.3: Gráfico do cruzamento das funções de intensidade.

para na sequência analisarmos o teste de comparação múltipla.

\begin{tabular}{|c|c|c|c|c|c|c|}
\hline \multirow[b]{2}{*}{ Ano de Ingresso } & \multicolumn{2}{|c|}{ Biológicas } & \multicolumn{2}{|c|}{ Exatas } & \multicolumn{2}{|c|}{ Humanas } \\
\hline & Ingressantes & Evasão & Ingressantes & Evasão & Ingressantes & Evasão \\
\hline 2000 & 1753 & 139 & 1994 & 419 & 3460 & 923 \\
\hline 2001 & 1880 & 104 & 2063 & 476 & 3515 & 768 \\
\hline 2002 & 2006 & 133 & 2262 & 382 & 3725 & 763 \\
\hline 2003 & 2065 & 101 & 2687 & 385 & 3772 & 693 \\
\hline 2004 & 2129 & 130 & 2828 & 371 & 3813 & 601 \\
\hline
\end{tabular}

Tabela 5.8: Comparação entre Áreas. 

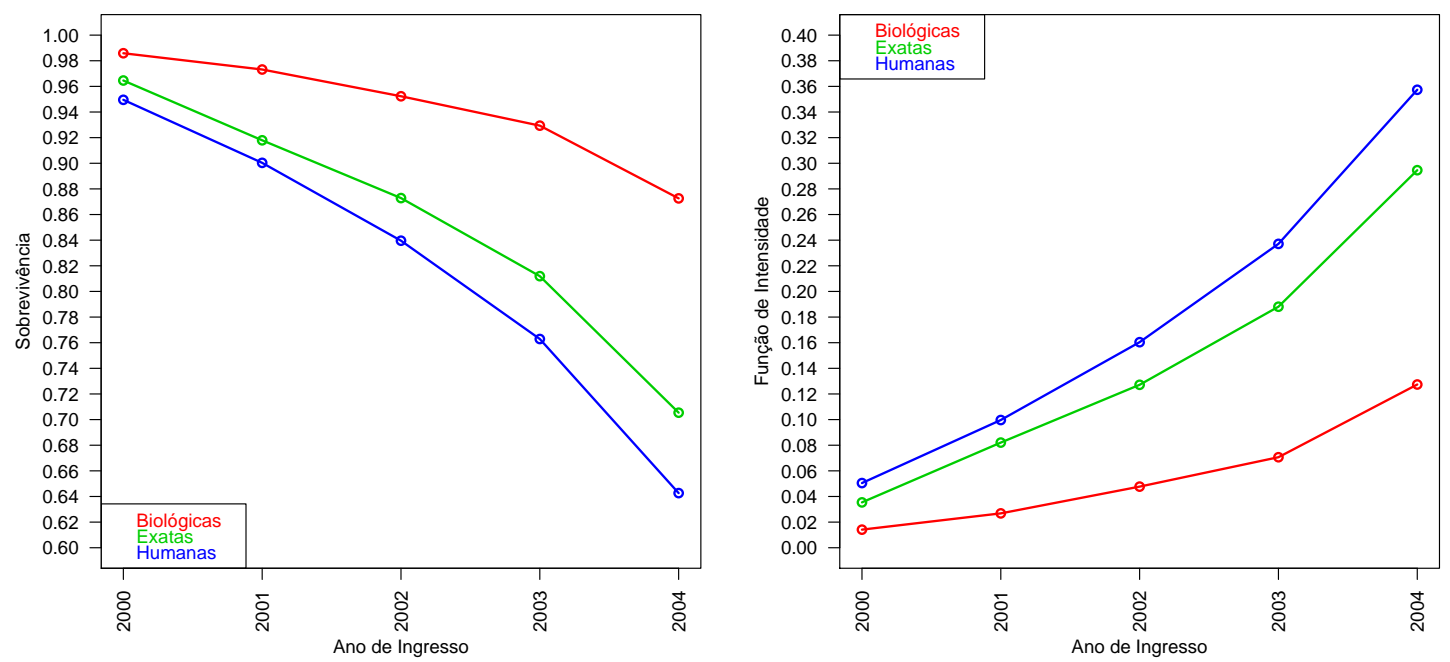

Figura 5.4: Gráfico das funções de intensidade por Área.

Na análise comparativa da evasão por área, observando a tabela (5.9), detectamos diferença significativa entre as áreas Biológicas, Exatas e Humanas para os três testes estatísticos ao nível de significância de $\alpha=0,05$. Agora o próximo passo é a comparação múltipla.

\begin{tabular}{lcc}
\hline & Estatística & p-valor \\
\hline LRP & 189,53 & $6,97 \times 10^{-42}$ \\
CVM & 1,74 & 0 \\
MLR & 214,4 & $5,84 \times 10^{-42}$ \\
\hline
\end{tabular}

Tabela 5.9: Resumo estatístico para comparação entre as áreas.

Através do estudo de comparações múltiplas, note que, temos diferenças significativas dois a dois para todas as estatísticas usadas (Cramér-von Mises, Log-rank Ponderado e Log-rank Modificado) ao nível de controle da FWER $\left(\alpha_{i}\right)$ descrito na tabela (5.10). O fato de todas as estatísticas encontrarem diferenças significativas é devido a proporcionalidade das funções de intensidade, observado na figura (5.4).

\begin{tabular}{|c|c|c|c|c|c|c|c|}
\hline \multirow[b]{2}{*}{ Comparação } & \multirow[b]{2}{*}{ FWER $\alpha_{i}$} & \multicolumn{2}{|c|}{ CVM } & \multicolumn{2}{|c|}{ MLR } & \multicolumn{2}{|c|}{ LRP } \\
\hline & & p-valor & Diferença & p-valor & Diferença & p-valor & Diferença \\
\hline Biológicas - Exatas & 0,01666667 & 0 & Sim & $6,12 \times 10^{-48}$ & Sim & $6,62 \times 10^{-49}$ & Sim \\
\hline Biológicas - Humanas & 0,025 & 0 & Sim & $4,61 \times 10^{-30}$ & Sim & $9,41 \times 10^{-33}$ & Sim \\
\hline Exatas - Humanas & 0,05 & $5,21 \times 10^{-7}$ & Sim & $1,23 \times 10^{-7}$ & Sim & $3,33 \times 10^{-5}$ & Sim \\
\hline
\end{tabular}

Tabela 5.10: Comparação 2 a 2 entre as áreas Biológicas, Exatas e Humanas. 



\section{Capítulo 6}

\section{Conclusões}

As aplicações para dados discretos na presença de censura são muito usados em diversas áreas, porém aplicados com modelos de probabilidades contínuos, ou seja, embora haja uma abundância de citações na literatura de estudos para dados com tempos de vida contínuos, há uma escassez de estudos na literatura aplicadas à dados com tempos de vida discreta. Para isto, enunciamos o modelo de intensidade para variáveis aleatórias discretas na presença de censura arbitrária, em especial a estatística de Cramér-von Mises proposta por Leão e Ohashi [16]. Neste trabalho propomos a estatística de Log-rank Modificado, que é uma modificação das estatística de Log-rank Ponderado proposto por Fleming e Harrington (1990) [5] e estendida por Leão e Ohashi [16] para $J$ populações.

Em nossa jornada deste longo trabalho, alcançamos os resultados esperados através de estudos de simulação. De fato a estatística de Cramér-von Mises têm maior poder em comparação com os demais testes propostos na literatura quando temos cruzamento severo das funções de intensidade. Mostramos que este teste é robusto em detectar diferenças nas funções de intensidade para dados discretos ou arredondados na presença de censura arbitrária. Mostramos também que uma modificação feita no teste de Log-rank clássico têm desempenho satisfatório para espaços amostrais finitos. Fato visto no capítulo (4) de simulação e no exemplo (5.2), em que o teste de Log-rank Modificado (MLR) têm ótimo resultado em tabelas de contingência, mesmo com cruzamento das funções de intensidade.

Resumindo os estudos de simulação, comprovamos que para dados com riscos proporcionais, quando estamos sob a hipótese nula e qualquer tipo de espaços amostrais $\mathcal{K} \subseteq \mathbb{N}$, as estatísticas propostas estão muito próximas da probabilidade nominal $(\alpha=0,05)$, ou seja, quando fixamos o nível de significância do teste, a probabilidade de se cometer um erro do tipo I está sob controle, mesmo para tamanhos amostrais variados e número de populações diferentes. Na avaliação da função poder empírica e espaços amostrais infinitos enumeráveis de categorias, temos que tanto Log-rank Ponderado quanto Cramér-von Mises têm desempenho satisfatório, na presença e ausência de censura. Quando avaliamos os espaços amostrais finitos de categorias $\mathcal{K}$, Log-rank Ponderado, Cramér-von Mises e Log-rank Modificado têm desempenho tão bom quanto o teste Qui-Quadrado, com a vantagem de utilizá-las para dados com censura arbitrária. 
Outro resultado obtido nas simulações, são para cruzamentos severos (riscos não proporcionais) e para diversas situações de cruzamento das funções de intensidade. Nesta situação a estatística de Cramér-von Mises têm uma taxa de convergência melhor em comparação com as demais, exceto para o caso de espaços amostrais finitos de categorias, no qual Log-rank Modificado tem melhor desempenho para um cruzamento das funções de intensidade e dois cruzamentos para apenas duas populações. No geral, Cramér-von Mises tem taxa de convergência maior.

O último estudo de simulação, foi para comparar as estatísticas propostas neste trabalho com as estatísticas enunciadas no livro de Klein e Moeschberger (1997)[15] para dados contínuos, que são Cramér-von Mises para dados contínuos (CCVM) e o de Renyi. Neste estudo, avaliamos as estatísticas primeiramente truncando completamente os dados contínuos, em seguida truncamos com uma casa depois da vírgula, duas casas, e por fim para os dados puramente contínuos. Estudamos estes dados em duas situações, a primeira com cruzamento suave das funções de intensidade e parâmetros das distribuições de probabilidade contínuas que fazem com ocorram uma concentração dos dados, ou seja, tenhamos um intervalo reduzido dos dados. O segundo experimento consiste em um cruzamento severo das funções de intensidade e parâmetros da distribuição de probabilidade contínuas que fazem com que tenha um intervalo maior de dados.

No primeiro experimento, notamos que o intervalo dos dados (concentração dos dados), fazem com que as estatísticas de Renyi e CCVM não se aproximem do nível de significância nominal $(\alpha=0,05)$. Com isso, retiramos elas das comparações na avaliação da função poder empírica. Avaliando as estatísticas propostas neste trabalho notamos que nas três situações temos comportamento mais satisfatório para a estatística de Cramér-von Mises em relação a de Log-rank Ponderado, exceto na primeira, em que ambos têm o mesmo comportamento. Como as estatísticas de Renyi e CCVM foram retiradas da comparação, não avaliamos nesta primeira situação o caso puramente contínuo.

No segundo experimento, notamos que o intervalo amplo dos dados, fazem com que as estatísticas de Renyi e CCVM ao contrário do primeiro experimento, se aproximem do nível de significância nominal adotado de $(\alpha=0,05)$. Com isso, foi possível compararmos as quatro estatísticas CVM, LRP, CCVM e Renyi na avaliação da função poder empírica. No caso de ausência de censura observamos que CCVM tem poder levemente maior nas quatro situações (truncamento total, 1 casa, 2 casas, puramente contínuo) em comparação com o teste de Renyi e CVM usando os pesos Gehan, Peto e Peto e Peto e Peto Modificado. Já LRP não se aproxima de 1 para tamanhos da amostra até 100, fato devido ao cruzamento severo das funções de intensidade. Agora, no caso de censura dos dados temos que CVM e Renyi têm maior poder e comportamentos similares que os demais. Notamos também, com censura dos dados, CCVM e LRP não se aproxima de 1 para tamanhos de amostra até 100, porém, temos que LRP dobra seu valor em comparação com o caso de ausência de censura, ao contrário de CCVM que diminui substancialmente seu valor. A censura à direita fixada em 100 faz com que o ponto do cruzamento seja antecipado, diminuindo o cancelamento das diferenças positivas e negativas entre as duas funções intensidade estimadas, fato este a causa da melhora do poder para LRP. Um balanço geral destes experimentos, é que CVM tem comportamento 
satisfatório para cruzamento severo das funções de intensidade independente dos parâmetros escolhidos para as distribuições de probabilidade, não ocorrendo o mesmo com as estatísticas propostas no trabalho de Klein e Moeschberger (1997)[15] que no primeiro experimento não tem bom comportamento sob a hipótese nula. O objetivo do estudo para dados truncados é que quando aumentamos as casas decimais, aumentamos bastante o número de categorias, mostrando assim que as estatísticas propostas têm bom desempenho para $\mathcal{K} \subseteq \mathbb{N}$ com $\mathcal{K}$ infinito enumerável de categorias.

Para concluirmos este trabalho, motivados pela escassez de estudos aplicados aos dados com tempo de vida discreta, a estatística de Cramér-von Mises enunciada, têm melhor desempenho frente aos demais testes encontrados na literatura, principalmente nos dados em que temos cruzamento severo das funções de intensidade. Nos dados contínuos dependendo da disposição dos dados a utilização das estatísticas enunciadas em Klein e Moeschberger (1997)[15] podem não ser adequadas, diferentemente da estatística de Cramér-von Mises, que tem desempenho satisfatório independentemente da disposição dos dados. Por fim, para os espaços amostrais finitos, em especial tabelas de contingência, modificações no teste de Logrank clássico têm comportamento tão bom quanto o clássico teste Qui-Quadrado, com a vantagem de ser utilizados em dados censurados. 



\section{Apêndice A}

\section{Apêndice}

\section{A.1 Parametrizações das distribuição de probabilidades utilizadas}

Definição A.1. Uma variável $X$ segue o modelo de Poisson de parâmetro $\lambda>0$, se sua função de probabilidade for dada por

$$
P(X=k)=\frac{e^{-\lambda} \lambda^{k}}{k !} \quad k \in \mathbb{N}
$$

Notação $X \sim \operatorname{Po}(\lambda)$. O parâmetro $\lambda$ indica a taxa de ocorrência por unidade medida.

Definição A.2. Seja X a variável aleatória que fornece o número de falhas até o primeiro sucesso. A variável X tem distribuição Geométrica com parâmetro $p, 0<p \leq 1$, se sua função de probabilidade é dada por

$$
P[X=j]=(1-p)^{j} p, \quad j \in \mathbb{N}
$$

Notação: $X \sim G(p)$.

Definição A.3. A variável aleatória X tem distribuição Exponencial de parâmetro $\lambda>0$, se sua função de densidade de probabilidade é dada por:

$$
f(x)= \begin{cases}1 / \lambda e^{-\frac{t}{\lambda}}, & \text { se } x>0 \\ 0, & \text { se } x \leq 0\end{cases}
$$

no qual o parâmetro $\lambda \geq 0$ é o tempo médio de vida e té um tempo de falha.

Observação A.1. Seja a variável aleatória definida em (A.3), então, calculamos a taxa de falha da distribuição Exponencial da seguinte forma:

Seja a taxa $h: \mathbb{R} \rightarrow \mathbb{R}$ com

$$
h(t)=\frac{f(t)}{1-F(t)}
$$


então,

$$
h(t)=\frac{(1 / \lambda) e^{-\frac{t}{\lambda}}}{e^{-\frac{t}{\lambda}}}=\frac{1}{\lambda} \in \mathbb{R}
$$

Definição A.4. Uma variável aleatória X tem distribuição Weibull se tiver função densidade de probabilidade dada por:

$$
f(x)= \begin{cases}\frac{\delta}{\alpha^{\delta}} x^{\delta-1} \exp \left[-\left(\frac{x}{\alpha}\right)^{\delta}\right], & \text { se } x \geq 0 \\ 0, & \text { se } x<0\end{cases}
$$




\section{Referências Bibliográficas}

[1] O. Aalen. Nonparametric inference for a family of counting processes. The Annals of Statistics, 6:701-726, 1978.

[2] O.O. Aalen, P.K. Andersen, Ø. Borgan, R.D. Gill, and N. Keiding. History of applications of martingales in survival analysis. Electronic Journal for History of Probability and Statistics, 5(1), 2009.

[3] P. K. Andersen, Ø. Borgan, R. D. Gill, and N. Keiding. Statistical models based on counting processes. Springer Verlag (New York), 1993.

[4] R.B. Davies. Algorithm as 155: The distribution of a linear combination of $\chi^{2}$ random variables. Journal of the Royal Statistical Society. Series C (Applied Statistics), 29(3):323-333, 1980.

[5] T.R. Fleming and D.P. Harrington. Counting processes and survival analysis, volume 8. Wiley New York, 1991.

[6] X. Gao, M. Alvo, J. Chen, and G. Li. Nonparametric multiple comparison procedures for unbalanced one-way factorial designs. Journal of Statistical Planning and Inference, 138(8):2574-2591, 2009.

[7] RD Gill. Censoring and Stochastic Integrals, Mathematical Centre Tracts 124. Amsterdam: Mathematisch Centrum, 1980.

[8] S.D. Grimshaw, J. McDonald, G.R. McQueen, and S. Thorley. Estimating hazard functions for discrete lifetimes. Communications in Statistics-Simulation and Computation, 34(2):451-463, 2005.

[9] P.L. Gupta, R.C. Gupta, and R.C. Tripathi. On the monotonic properties of discrete failure rates. Journal of Statistical Planning and Inference, 65(2):255-268, 1997.

[10] D.P. Harrington and T.R. Fleming. A class of rank test procedures for censored survival data. Biometrika, 69(3):553, 1982.

[11] Y. Hochberg. A sharper Bonferroni procedure for multiple tests of significance. Biometrika, 75(4):800, 1988. 
[12] Y. Hochberg and D. Rom. Extensions of multiple testing procedures based on Simes' test. Journal of Statistical Planning and Inference, 48(2):141-152, 1995.

[13] S. Holm. A simple sequentially rejective multiple test procedure. Scandinavian Journal of Statistics, 6(2):65-70, 1979.

[14] D. Karlis and V. Patilea. Confidence intervals of the hazard rate function for discrete distributions using mixtures. Computational statistics \& data analysis, 51(11):53885401, 2007.

[15] J.P. Klein and M.L. Moeschberger. Survival analysis: techniques for censored and truncated data. Springer Verlag, 2003.

[16] D. Leão and A. Ohashi. On the discrete cramér-von mises statistics under random censorship. arXiv preprint arXiv:1201.2377, 2012. Submetido.

[17] N. Mantel. Chi-square tests with one degree of freedom; extensions of the MantelHaenszel procedure. Journal of the American Statistical Association, 58(303):690-700, 1963.

[18] R. Pelissari. Análise empírica de dados multinomiais. Master's thesis, Universidade de São Paulo (USP). Instituto de Ciências Matemáticas e de Computação, 2009.

[19] R Development Core Team. R: A Language and Environment for Statistical Computing. R Foundation for Statistical Computing, Vienna, Austria, 2011. ISBN 3-900051-07-0.

[20] S.K. Sarkar. Some probability inequalities for ordered MTP2 random variables: a proof of the Simes conjecture. Annals of Statistics, 26(2):494-504, 1998.

[21] D.M. Stablein and IA Koutrouvelis. A two-sample test sensitive to crossing hazards in uncensored and singly censored data. Biometrics, 41:643-652, 1985.

[22] Q. Yu. A note on the proportional hazards model with discontinuous data. Statistics \& probability letters, 77(7):735-739, 2007. 